
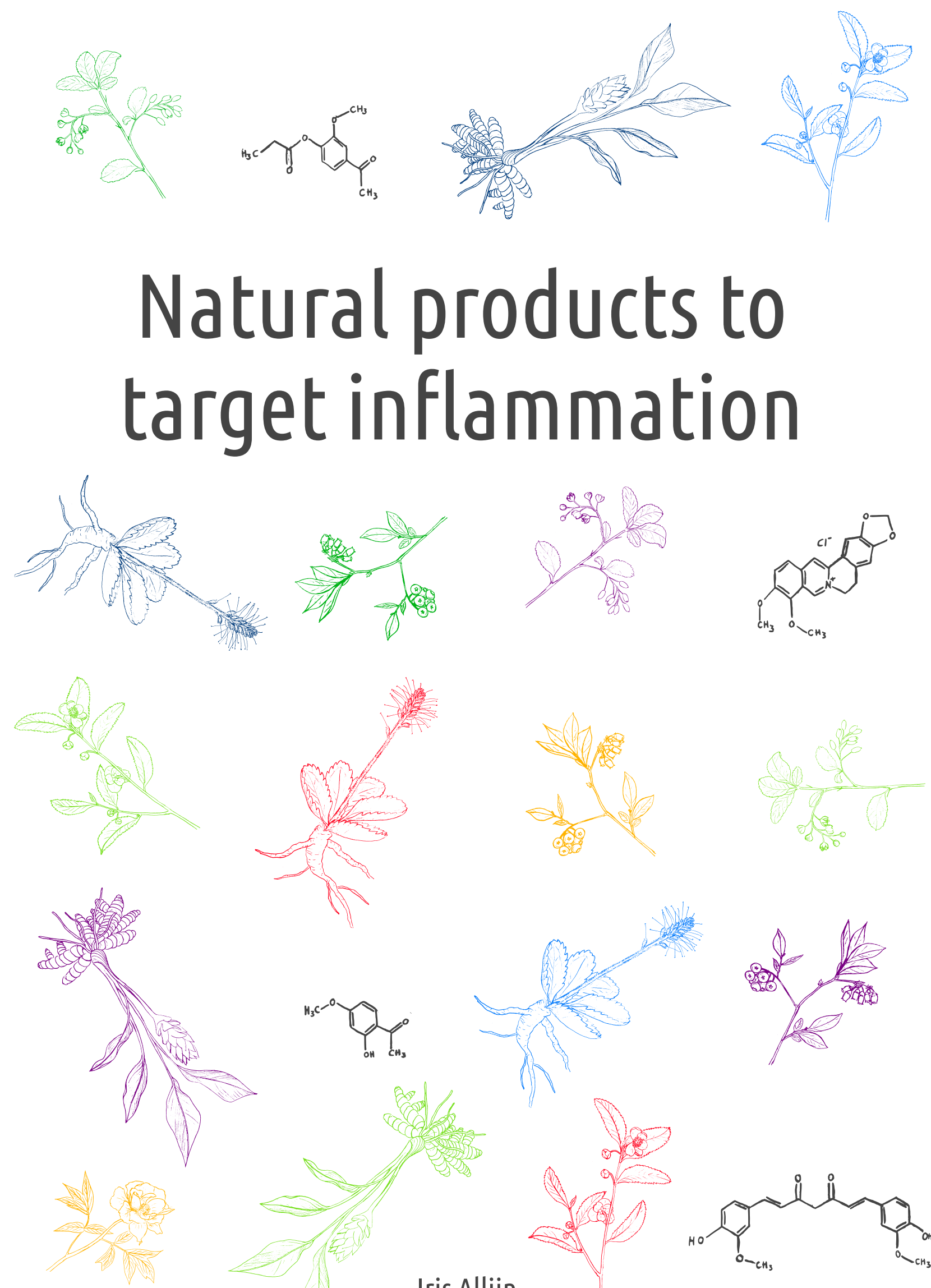

Iris Allijn
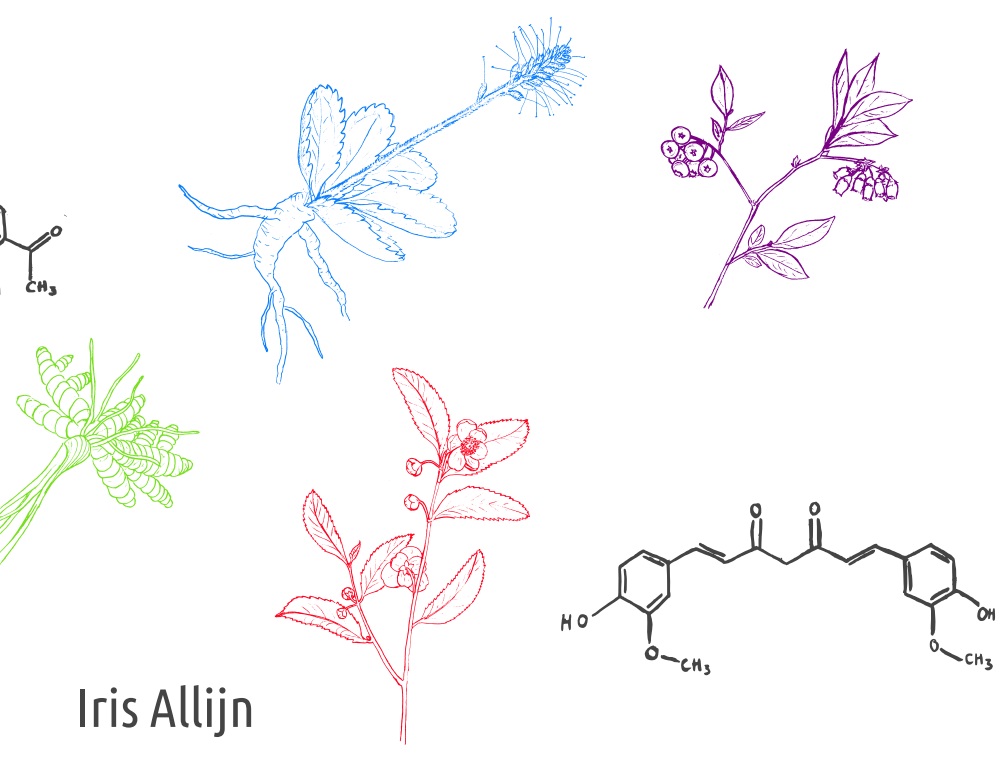


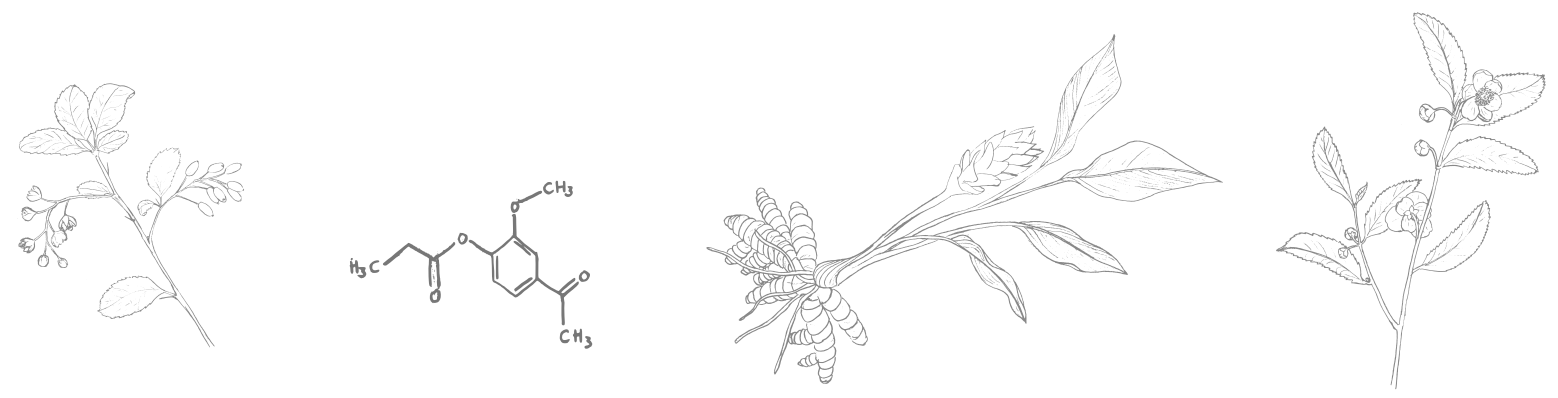

\section{NATURAL PRODUCTS TO TARGET INFLAMMATION}
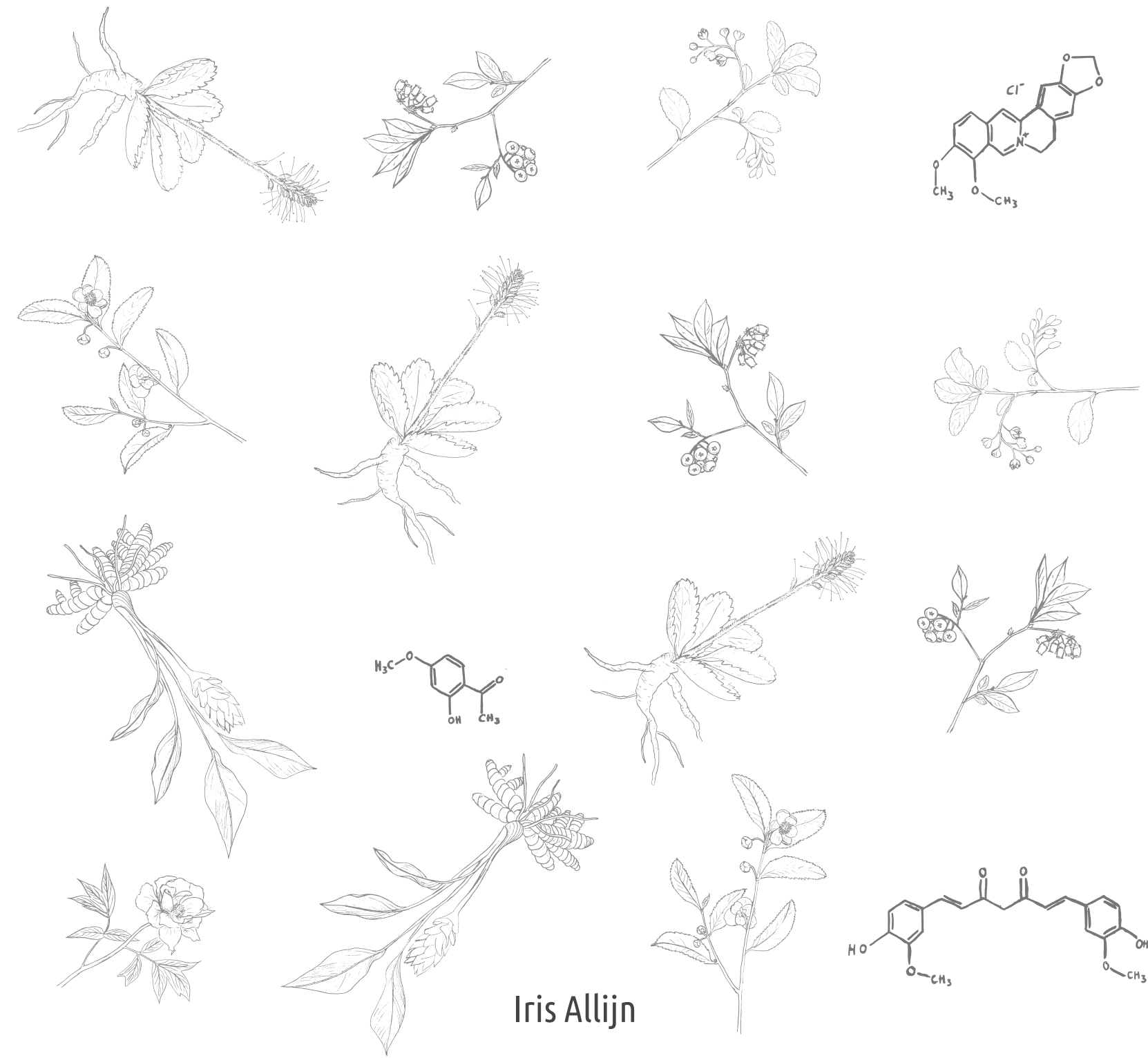


\section{Graduation committee}

Chairman \& secretary

Promotor

Assistant promotor

Members
Prof. dr. J.W.M. Hilgenkamp

Prof. dr. G. Storm

Prof. dr. R.M. Schiffelers

Prof. dr. R. Passier

Prof. dr. J.F.J. Engbersen

Prof. dr. D.W. Grijpma

Prof. dr. A.M.J. Ragas

Dr. C.J. Beukelman
University of Twente

University of Twente

University Medical Center Utrecht

University of Twente

University of Twente

University of Twente

Radboud University Nijmegen

Utrecht University

The research reported in this dissertation was funded by NanoNextNL (03D.11), the Dutch national research and technology programme for micro and nano technology.

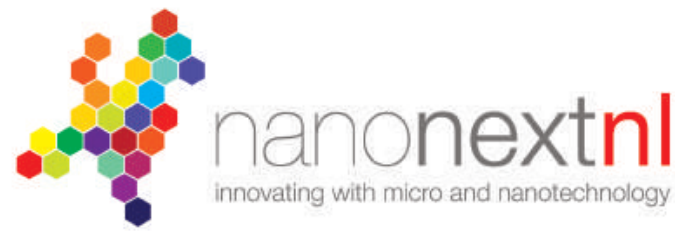

The research reported in this dissertation was carried out on three continents at eight different institutes:

UNIVERSITY OF TWENTE.

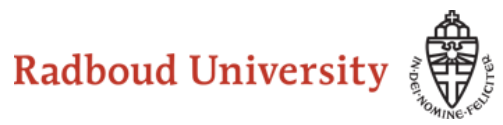

(C) 2016 Iris Eva Allijn

Cover design and various graphics by Tirza Allijn

LTEX template: arsclassica, classicthesis

Printed by Wöhrmann Print Service

ISBN: 978-90-365-4205-0

DOI: $10.3990 / 1.9789036542050$

All rights reserved. No part of this work may be reproduced, stored in a retrieval system, or transmitted in any form or by any means, electronic, mechanical, photocopying, recording, or otherwise, without prior permission from the copyright owner. 


\title{
NATURAL PRODUCTS TO TARGET INFLAMMATION
}

\author{
DISSERTATION
}

to obtain

the degree of doctor at the University of Twente,

on the authority of the Rector Magnificus

Prof. dr. H. Brinksma

on account of the decision of the graduation committee,

to be publicly defended

on Wednesday, 19th of October 2016 at 16:45

by

Iris Eva Allijn

born on October 30, 1986

in Amsterdam, The Netherlands 
This thesis has been approved by:

Prof. dr. G. Storm, promotor

Prof. dr. R.M. Schiffelers, co-promotor 


\section{ACKNOWLEDGMENTS}

The research in this dissertation was carried out on three continents at eight different institutes. I want to thank all people who contributed to the experiments, discussions and happy times:

University of Twente - my base lab. Karin (P), we shared an office for 4 years. This resulted in very inefficient writing, but it was more than worth it! Thank you for all the amusing 'sneaky' coffee breaks for good conversations and discussions! Daphne, thanks for your support in finishing this dissertation! Karin $(\mathrm{H})$, thank you for helping with all the paper work, and answering all my questions. And then often answering them again! Tessa, it was always good to see you again on all NanoNextNL events, thank you for being there. Furthermore, I want to thank Acarilia, Bas, Denys, Dirk, Dwi, Erwin, Frits, Guoying, Hetty, Jai, Johan, Jonas, Jos, Karin (R), Lydia, Marc, Natalia, Nick, Niels, Pia, Praneeth, Rachel, René, Ruchi, Sale, Tony, Wim, Yvonne and Zlata for the corridor discussions in-between experiments. Utrecht University my extra lab and base of my promotor. Gert, thank you for getting me as your first PhD in your new Twente lab. You were always very critical. Thank you for keeping me sharp. Furthermore, Barbara, Burcin, Kees, Linda, Louis, Merel, Mies and Réka thank you for helping me out in the lab.

University Medical Center Utrecht - my second extra lab and base of my co-promotor. Raymond, thank you for always being optimistic! Marco, Roy, Işil, Steven, Susan and Suzanne thank you for showing me around and helping me out in your lab.

Amsterdam Medical Center - my third extra lab. Menno, thank you for having me in your lab when we were still building our lab in Twente. I learned a lot (about macrophages) from you in the first year. Furthermore, Annette, Esther, Jan, Marieke, Marten and Tom thank you for your support in the lab.

Mt Sinai New York City - where it all began. Willem, thank you for all your enthusiasm for nanomedicine and research, it was very contagious. David, I think we were a great team. I learned a lot from you! And also I want to thank Aneta, Anita, Jun, Mark, Nicole, Wei for the experimental help and fun in and outside the lab.

Radboud University Nijmegen. Rik and Ad, thank you for giving me insights into the environmental sciences, which resulted in chapter 7 .

University of Applied Sciences Utrecht. Stefan, thank you for your additional experiments and pleasant collaboration for chapter 3 . 
National University of Singapore. Bertrand and Jiong-Wei, thank you for the pleasant collaboration for chapter 6 .

Dad, as a biologist, you always showed me the beauty of life, and I guess that's why I studied biomedical sciences in the first place.. Mom, I'm so grateful for letting me go and explore life. Hannah, Rachel and Tirza, thank you for just always being there.

Frank, Quin and Vera $\odot \odot$

Iris Allijn 


\section{SUMMARY}

Most Western lifestyle diseases such as type 2 diabetes mellitus, cardiovascular disease and cancer have a chronic inflammatory process at its base. Therefore, inflammation is an important therapeutic target. Due to their potency, steroidal drugs dominate the current treatment of inflammatory disorders. However, steroidal drugs can also exert a broad range of side effects. This thesis looks at the field of 'natural products' to find alternatives. A natural product is a molecule produced by a living organism. We performed a systematic literature review in which we ranked the anti-inflammatory performance and bioavailability of 102 natural products. Although many of these products do appear to show promise, bioavaibility is often poor. Furthermore, many studies focus on single inflammatory pathways and single molecules, making meaningful comparison difficult. Therefore, we compared the response of multiple inflammatory mediators to eight well-known natural products. The steroidal drug prednisolone was included as golden standard. We identified curcumin and berberine as promising alternatives to steroidal drugs. One strategy to improve the low aqueous solubility of natural products is by loading them into nanoparticles. Low-density lipoprotein (LDL) has many attractive properties with regard to inflammation and bioavailability. For that reason, we developed an effective new method to use LDL as a nanoparticle for drug delivery. Curcumin, with its favorable anti-inflammatory profile but poor bioavailability, is a prime candidate for encapsulation in nanoparticles. In an in vitro comparison of LDL and three other nanoparticles, the former achieved the highest relative curcumin loading capacity. Berberine has been described to have cardioprotective in addition to anti-inflammatory properties. To enhance the aqueous solibility, we prepared a liposomal formulation and showed that berberine, when properly delivered, protects cardiac function after myocardial infarction in mice. Human safety and toxicity is an important concern in the development of pharmaceuticals. However, there is still little attention for their (aquatic) environmental safety and the indirect impact on human health after exposure. To emphasize this, we compared the environmental impact of berberine and prednisolone. To conclude, we have shown that natural products, especially when encapsulated in nanoparticles, can indeed serve as potential alternatives for steroidal drugs in inflammatory diseases.

Chapter 1

Chapter 2

Chapter 3

Chapter 4

Chapter 5

Chapter 6

Chapter 7

Chapter 8 



\section{SAMENVATTING}

De meeste ziekten in de Westerse samenleving, zoals type 2 diabetes, hart- en vaatziekten en kanker, worden gekenmerkt door chronische ontsteking. Steroïden domineren de huidige behandeling, zijn krachtig maar hebben veel bijwerkingen. In dit proefschrift worden 'natuurlijke verbindingen' onderzocht om alternatieven te vinden. Een natuurlijke verbinding is een molecuul geproduceerd door een levend organisme. In een systematische literatuurstudie, hebben we de anti-inflammatoire werking en de biologische beschikbaarheid van 102 natuurlijke verbindingen gerangschikt. Hoewel veel van deze verbindingen inderdaad een zekere anti-inflammatoire acitviteit lijken te vertonen, is de biologische beschikbaarheid in de meeste gevallen een probleem. Vergelijking wordt bemoeilijkt doordat de meeste studies slechts een enkele inflammatoire factor in combinatie met een enkel molecuul testen. We hebben daarom een eigen studie uitgevoerd waarin we acht bekende natuurlijke verbindingen op basis van meerdere inflammatoire factoren vergeleken met het steroïd prednisolone als gouden standaard. Curcumin en berberine bleken veelbelovende alternatieven te zijn. Om natuurlijke verbindingen beter te laten opnemen door het lichaam, kunnen ze ingebracht worden in nanodeeltjes. We hebben in eerste instantie het lipo-proteïne LDL, een lichaamseigen deeltje met aantrekkelijke eigenschappen onderzocht. Allereerst hebben we een effectieve nieuwe methode ontwikkeld van bereiding. Curcumin, met haar gunstige anti-inflammatoire werking maar slechte biologische beschikbaarheid, is een perfecte kandidaat voor belading in een nanodeeltje. In een in vitro studie hebben we LDL en drie andere nanodeeltjes vergeleken. LDL bleek de hoogste relatieve ladingscapaciteit te hebben. Berberine, de andere aantrekkelijke verbinding, hebben we in een liposoom gebracht. We hebben aangetoond dat liposomaal berberine de hartfunctie bij muizen beschermt na een myocardiaal infarct. Als achtergrondstudie hebben we de milieu-effecten van berberine en prednisolone vergeleken. We vestigen hier graag de aandacht op, omdat bij de ontwikkeling van geneesmiddelen het (aquatisch) milieu nog maar nauwelijks een factor is. Dit heeft indirecte gevolgen voor de volksgezondheid. Onze algemene conclusie luidt dat natuurlijke verbindingen, vooral wanneer ze omhuld zijn door een nanodeeltje, inderdaad kunnen dienen als mogelijke alternatieven voor steroïde geneesmiddelen voor inflammatoire ziekten.

Hoofdstuk 1

Hoofdstuk 2

Hoofdstuk 3

Hoofdstuk 4

Hoofdstuk 5

Hoofdstuk 6

Hoofdstuk 7

Hoofdstuk 8 



\section{CONTENTS}

I GENERAL INTRODUCTION I

1.1 Inflammation $I$

1.2 Metabolic Syndrome 3

1.3 Natural products 6

1.4 Nanomedicine 7

2 Systematic REVIEW II

2.1 Introduction 11

2.2 Article selection 12

2.3 Structural classification 13

2.4 Oral bioavailability 31

2.5 Anti-inflammatory activity 34

2.6 Best performing and most promising natural products 40

2.7 Predicting anti-inflammatory activity of natural products $\quad 45$

2.8 Discussion 46

2.9 Future perspectives and conclusions 50

3 BENCHMARKING NATURAL PRODUCTS 59

3.1 Introduction 59

3.2 Results 64

$3 \cdot 3$ Discussion 71

3.4 Materials \& Methods 72

4 LDL AS DRUG CARRIER 83

4.1 Introduction 84

4.2 Results and discussion 85

4.3 Conclusions 95

4.4 Materials and methods 96

5 CURCUMIN NANOFORMULATIONS 107

5.1 Introduction 108

5.2 Results and discussion 111

$5 \cdot 3$ Conclusion 115

5.4 Materials and methods 116

6 BERBERINE 123

6.1 Introduction 124

6.2 Results and discussion 125

6.3 Conclusions 130

6.4 Materials and methods 130

7 ENVIRONMENTAL RISK ASSESSMENT OF PHARMACEUTICALS 139

7.1 Introduction 140

7.2 Methodology 142 
$7 \cdot 3$ Results 147

7.4 Discussion 151

7.5 Conclusion 152

8 SUMMARIZING DISCUSSION

159 


\section{GENERAL INTRODUCTION}

\subsection{INFLAMMATION}

The very first documented recognition of inflammation goes back to the 1st century AD, when Aulus Cornelius Celsus noted that inflammation has four key elements; rubor (redness), calor (heat), tumor (swelling) and dolor (pain). In the 1850 o a fifth element of inflammation was introduced by Rudolf Virchow; functio laesa (loss of function) $[11,24]$. Not much later, in 1899, the concept of phagocytosis was introduced by Elie Metchnikoff, marking the beginning of knowledge on innate immunity [24]. A full century later, a tremendous amount of research has been performed on inflammation and its diseases associated with uncontrolled inflammatory reactions.

In general, inflammation is the mechanism that responds to disturbances like tissue damage and infection to ultimately restore functionality and homeostasis $[17,1]$. A first reaction is the arteriolar vasoconstriction by smooth muscle cells, followed by arteriolar vasodilation, which leads to an increased blood flow and local hyperemia [1]. As a reaction, white blood cells and plasma proteins extravasate into these tissues and subsequently the resident tissue cells are activated [24, 1]. This inflammatory response is a delicate balance between positive and negative feedback loops and additional chemical checkpoints to promote tissue repair [5] A cell has four possible states, basal (normal homeostasis), stressed (disturbed homeostasis), apoptotic (resolution of inflammation) and necrotic (resolution has failed) [17]. Regulated clearance of apoptotic cells by phagocytosis leads to the inhibition of the inflammatory response and is necessary for successful resolution of inflammation [1].

\subsubsection{The acute inflammatory response}

An acute and controlled inflammatory response is beneficial for the body [17]. Triggered by infection or tissue injury, the innate immune response is activated [5]. Exogenous inducers include pathogen associated molecular patterns (PAMPs), like lipopolysaccharide (LPS), and endogenous inducers include reactive oxygen species (ROS) produced by stressed, damaged or malfunctioning tissues, giving rise to oxidized low-density lipoprotein (oxLDL), oxidized RNAs and other oxidized 
proteins [17]. Recognition is mediated by macrophages, residing in the affected tissue [24, 17]. This leads to the production of, among more, chemokines (like IL-8), cytokines (like TNF- $\alpha$ and IL-6) and eicosanoids (like $\mathrm{PGE}_{2}$ ) and ROS [24, 17]. These inflammatory mediators amplify and sustain the inflammatory response [24]. Subsequently, the activated local epithelial and mesenchymal cells respond to these pro-inflammatory mediators and start participating in propagating the inflammatory response by generating these same signals [24]. As a response to this, there is an increased blood flow and enhanced vascular permeability [1].

Acute

inflammation is beneficial for the body. For in depth reading on the consequences of failure of resolution, please see $R$.

Medzhitov, 2008 [17] and Buckley et. al., 2014 [5].

A successful acute inflammatory response includes the elimination of the inflammatory agent and is followed by resolution of the inflammation and repair of the damaged tissue [17]. For the resolution of inflammation it is crucial that endogenous programs switch from proinflammatory mediators towards pro-resolving mediators like lipoxins [17] and lipid mediators [1]. Furthermore, signaling pathways stimulating leukocyte survival have to be switched off and recruited inflammatory cells have to undergo apoptosis and have to be cleared by phagocytic cells. And, finally, the transformation of pro-inflammatory macropahges (M1 type) into pro-resolution macrophages (M2 type) has to take place [1]. If the acute inflammatory response fails to eliminate the cause of the inflammation, the inflammatory process continues, acquiring new characteristics and eventually evolving into chronic inflammation [17]. Timely resolution of inflammation is thus of great importance to prevent further tissue dysfunction or damage [1].

\subsubsection{Chronic inflammation and disease}

Macrophages have a high plasticity, with the extremes of pro-inflammatory (M1) and anti-inflammatory

(M2). The same macrophages can initially promote and in a later stage resolve inflammation. For in depth reading, see Stöger et. al., 2010 [28] and Allavena and Mantovani, 2012
Acute inflammation can become detrimental when it is dysregulated [17], uncontrolled or unresolved and it can give rise to chronic inflammation [1]. Chronic inflammation appears to be associated with a homeostatic imbalance of one or more physiological systems, yet, in contrast with acute inflammation, less is known about this process. Chronic inflammation does not seem to be initiated by an acute trigger [17]. Nonetheless, it is very clear that inflammation brings together pathophysiological mechanisms underlying many chronic diseases [24, $17,1]$. Many chronic diseases maintained by low grade lingering inflammation [24].

Cardiovascular disease (CVD) is well-known for the chronic inflammation at the base of the disease $[17,1]$. CVD is among the leading causes of death worldwide [21]. The chronic pro-inflammatory state and chronic subclinical vascular inflammation, ultimately lead to the development of atherosclerosis [13]. Complications of atherosclerosis, like thrombosis or plaque disruption involve inflammation as well [24]. 
Rupture of atherosclerotic plaques leads to thrombus formation and is the most common cause of myocardial infarction (MI) in humans [14, 29]. Although there has been tremendous progress with cholesterollowering drugs, CVD still persists, and thus there are unmet needs for new therapeutics [14], which might be found in the field of natural products.

Type 2 diabetes mellitus (T2DM) is, like CVD, also among the leading causes of death worldwide [21], and has chronic inflammation at its base as well [17]. Chronic inflammation, maintained by macrophages in adipose tissue, contributes to insulin resistance and the development of obesity [28, 13]. Other diseases in which chronic inflammation plays an important role include arthritis, asthma [17, 1], cancer $[17,4]$, and autoimmune diseases [17]. ROS may have a role in the development of these chronic inflammatory diseases [1].

\subsection{METABOLIC SYNDROME}

Metabolic syndrome (MetSyn) is the name for a group of metabolic conditions that occur together and promote the development of CVD and T2DM $[14,21]$. These conditions include insulin resistance, central or visceral obesity, atherogenic dyslipidemia, hypertension [10, 13, 21], endothelial dysfunction [10, 13] and chronic stress [13] (Figure 1.1). In MetSyn the body is in a state of chronic low grade inflammation [13]. MetSyn as a disease entity remains debated [6], however the concept has practical advantages and allows easier identification of patients at higher risk of the development of CVD or T2DM [10].

The origins of MetSyn lie in 1923, when E. Kylin demonstrated that there is an association of hypertension with hyperglycemia and gout. Two decades later, in 1947, J. Vague described the association of metabolic abnormalities with visceral obesity in CVD and T2DM. Again two decades later, in 1965, a syndrome was described by Avogaro and Crepaldi which comprised hypertension, hyperglycemia and obesity [13]. These conditions were first introduced as 'metabolic syndrome' in 1975 by Haller and Hanefeld [21]. However it took until 1988 for MetSyn to gain momentum, when it was discussed as 'Syndrome $X^{\prime}$ or 'insulin resistance syndrome' in the Banting Lecture of G.M. Reaven $[14,13]$. Since the late 1990s, multiple attempts for a single definition of MetSyn have started from institutes like the World Health Organization (WHO) and the International Diabetes Federation (IDF). This resulted in several overlapping however different definitions [13].

Because of these differences in definition, it is hard to measure the exact prevalence of MetSyn. The IDF however, estimates that one quarter of the adult world population is affected [13]. Nevertheless, regardless

MetSyn is a clustering of metabolic disturbances including insulin resistance, visceral obesity, dyslipidemia and hypertension. These promote CVD and T2DM. For in depth reading, see $O^{\prime}$ Neill and O'Driscoll, 2015 [21] and Kaur, 2014 [13]. 
of definition, the prevalence of MetSyn is increasing with body mass index (BMI) and age [21]. There is great variation in age of onset for people with similar risk profiles, which suggests that not only environmental, but also genetic factors play a role [13]. Furthermore, men are more susceptible to MetSyn than women, which is caused by the difference of fat distribution. Women tend to have more subcutaneous fat, whereas men more visceral fat. The latter is associated with insulin resistance, which is possible caused by increased glucocorticoid receptor and inflammatory cytokine secretion which is not present in subcutaneous fat [14]. MetSyn increases the risk of developing T2DM 5-fold, and CVD 2-3 fold $[14,13,21]$ and can be seen as a growing epidemic [21].

\subsubsection{Manifestation of metabolic syndrome}

The main environmental risk factors for developing MetSyn are physical inactivity and a high calorie diet $[14,21]$. The metabolic syndrome traits are influenced by genetic factors with a heritability of about $40 \%$, some factors like HDL levels and obesity have even a heritability of about $70 \%$ [14]. In one of these main traits, i.e. insulin resistance, adipose, muscle and liver cells do not respond adequately to insulin, resulting in high blood glucose levels [10]. Normally, insulin suppresses lipolysis in adipocytes and degrades apolipoprotein B on (V)LDL, thus impaired insulin signaling increases free fatty acids (FFA) levels and enhances the production of VLDL [13]. This leads to dyslipidemia, which is characterized by lipid abnormalities and perturbations in lipid metabolism and biological activities [13] with high plasma triglycerides, low HDL levels and increased LDL levels [10]. Glucose intolerance and dyslipidemia are often associated with hypertension [13]. Systemically, MetSyn affects the kidneys, liver, skin, eyes, reproductive system, cardiovascular system and certain cancers [13].

On the molecular level, macrophages infiltrated in adipose tissue start overproducing adipocytokines and other biologically active metabolites, including TNF- $\alpha$, IL-6 and C-reactive protein (CRP) [14, 13]. This results in localized inflammation, however propagates an overall systemic inflammatory response. TNF- $\alpha$ inhibits the insulin receptor substrate 1 signaling pathway, enhancing the insulin resistance. Furthermore, high plasma levels are positively associated with body weight and waist circumference, and negatively with HDL cholesterol levels, enhancing even more the pathologies of MetSyn. IL-6 is linked with a high BMI, low HDL cholesterol levels and impairs insulin sensitivity. Furthermore, it is a major contributor of CRP production in the liver. CRP, as well, is linked with increased waist circumference, insulin 
resistance and BMI. [13] Altogether, these inflammatory mediators sustain and enhance the traits of MetSyn.

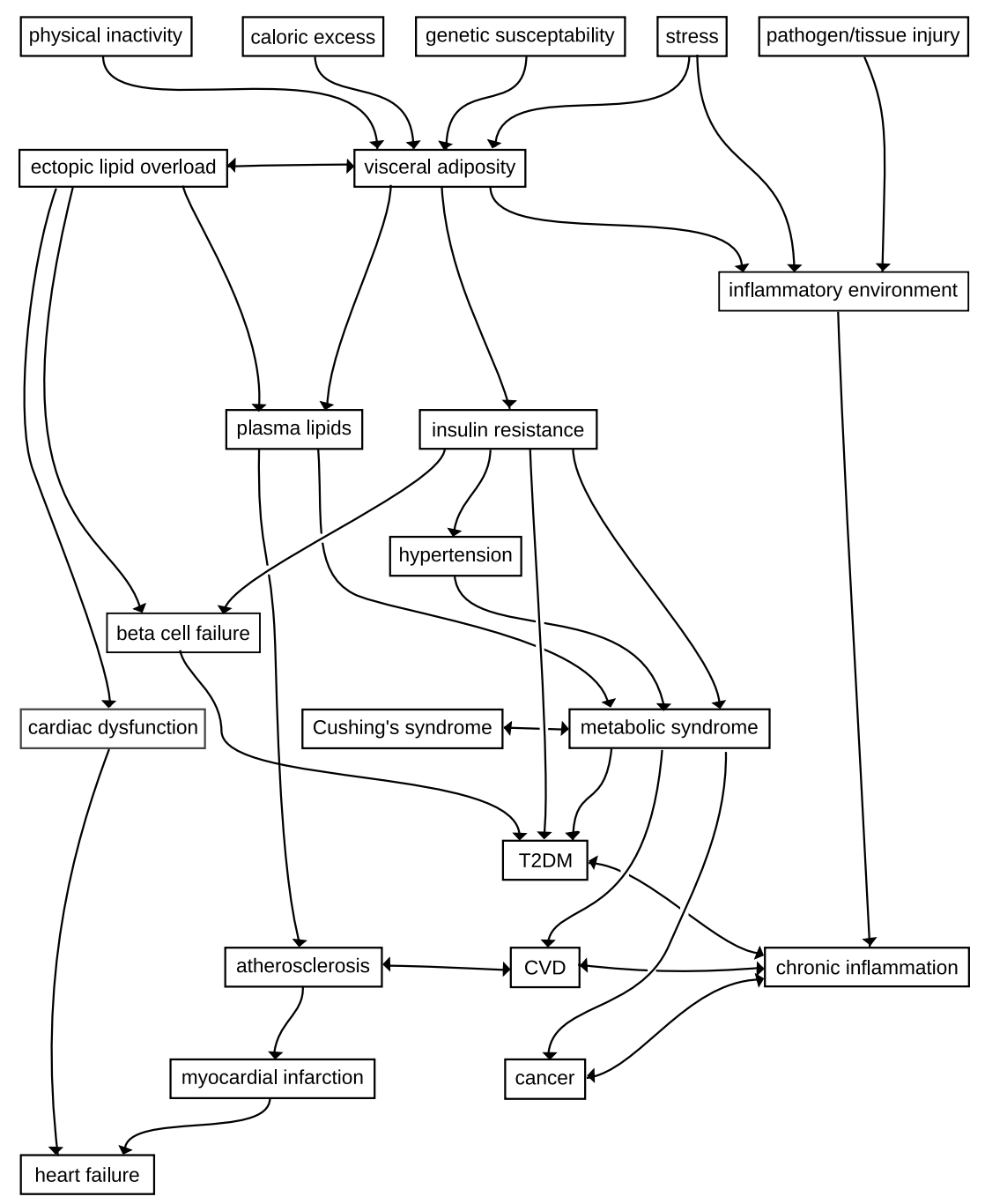

Figure 1.1: Metabolic syndrome schematic. Causes and consequences of metabolic syndrome are closely related to inflammation and chronic inflammatory diseases.T2DM = type 2 diabetes mellitus, CVD = cardiovascular disease. Schematic based on Lusis, 2008 [14] and Kaur, 2014 [13]. 


\subsubsection{Metabolic syndrome and disease}

The homeostatic disturbances of MetSyn, enhance the risk of developing chronic inflammatory diseases like cancer [21], CVD [14] and T2DM [13]. Tackling MetSyn would be beneficial in reducing the development of these diseases and hence the economic burden they entail. Such an approach would be multidisciplinary, tackling each and every factor of MetSyn separately. Examples include lowering LDL cholesterol levels using statins and weight loss diet programs [13].

Chronic use of glucocorticoids like prednisolone, can increase lipolysis or lipid oxidation and can cause visceral fat accumulation in individuals with genetic predisposition [13]. This can lead to Cushing's Syndrome, in which fat redistribution is one of the main traits. The central fat accumulation in Cushing's Syndrome combined with glucose intolerance and hypertension is similar to MetSyn [15]. Glucocorticoids are associated with an extensive number of physiological changes in MetSyn [19] and there is ever increasing evidence that glucocorticoid signaling contributes to the pathogenesis of MetSyn and obesity. Nevertheless, glucocorticoids are used in the therapy of MetSyn because of the potent anti-inflammatory activity outweighs the metabolic effects [15]. Anti-inflammatory activity without the metabolic effects, or another spectrum of anti-inflammatory activities are therefore of high pharmaceutical interest. Compounds with such profiles might be found within the field of natural products.

\subsection{NATURAL PRODUCTS}

Natural products are chemical compounds or substances that are produced by living organisms like fungi, plants and bacteria [2]. Molecular types of natural products include alkaloids, fatty acids, steroids, terpenoids, flavonoids and stilbenes [8]. Natural products are found in common food like cocoa, olive oil, wine and coffee [29] and are often important metabolites in plants with key roles in antibiotic mechanisms, defense against herbivores, protection against UV radiation, nutrition or growth of the plant [26].

Natural products are single molecules derived from a natural source like plants, fungi or marine organisms
Traditional medicines, in particular derived from plants, have been used for thousands of years [22]. The earliest records are from around thousand plant-based substances in Mesopotamia, 2600 BC, including cedar, cypress, licorice, myrrh and poppy juice. These plants are still in use today [20]. The Ebers Papyrus, dating from $1500 \mathrm{BC}$, is the largest record of ancient Egyptian medicine, which documents over seven hundred plant based drugs [20, 9]. A bit later, in $1100 \mathrm{BC}$, the Chinese recorded their prescriptions on natural products as well in 
the Materia Medica, and also the Indian Ayurvedic system dates from before $1000 \mathrm{BC}$. The Greeks and Romans contributed to the use of herbal medicine in the ancient Western World [20]. Nowadays, around $80 \%$ of the global populations still relies on the use of botanical drugs [27].

Natural products show a far greater structural diversity than synthetic drugs $[8,26]$, and hence natural products play an important role in drug discovery [8]. An well-known example is the discovery of aspirin, which is derived from the known analgesic and antipyretic properties of the bark of the willow tree, which was used by the Greeks and Romans in $\sim 400$ BC [8]. Today, around half of the pharmaceuticals in use are derived from natural products [23]. Presently, natural products are especially known for their anti-oxidant properties and are under investigation because of their beneficial impact on countering (chronic) inflammation [29]. To increase their potency, target drug delivery is an attractive strategy. This can be achieved by encapsulation into nanosized materials.

\subsection{NANOMEDICINE}

The prefix 'nano' is derived from the Greek ' $v \alpha v o \zeta$ ' (nános) and refers to the application of nanotechnology in medicine [12], the unit prefix 'nano' means one billionth or $1^{-9}$. Nanomedicine is a key discipline of the $21^{\text {st }}$ century [18], including nanodiagnostics, nanopharmaceuticals, nanorobotis and nanosurgery [12]. Although it is a young science, which has only been actively researched since the 1990s [18], the roots of nanotechnology go far back in time.

Colloidal gold and silver nanoparticles, used in church glass to give its specific coloration when illuminated, dates from the $4^{\text {th }}$ century AD. The use of transmission electron microscopy (TEM), has advanced the discovery of nanoparticle use, however, was not invented up until the early 1930s [18]. The nanotechnological approach was first framed by Richard Feynman in 1959, where he describes a new field of physics with an enormous amount of technical applications [7], however, the expression 'nano' was not yet used.

The start of the field of nanopharmaceuticals (or nanocarriers), was between 1955 with reporting a polymer-drug conjugate by H. Jatzkewitz and 1965, with the discovery of liposomes by A. D. Bangham. In 1972, the first albumin-based nanoparticle was reported by U. Scheffel and can be seen as the precursor for protein-based nanoparticles. In 1975, H. Ringdorf conceptualized targeted drug conjugates and described key principles which are still valid and in use today. [25] The field made a gigantic leap with the discovery of enhanced vascular
Drug development programs often use natural products as lead compounds because of their structural variety.
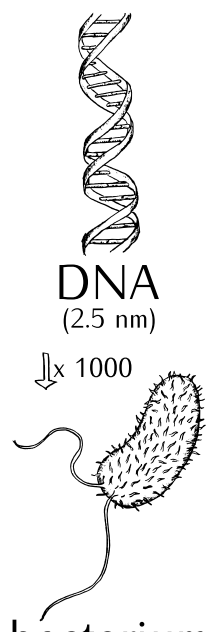

bacterium

$(2.5 \mu \mathrm{m})$

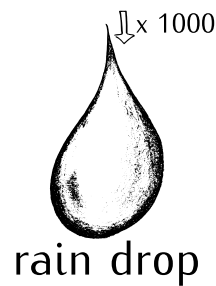

$(2.5 \mathrm{~mm})$ 
Nanomedicines are therapeutics based on nanoparticles. For in depth reading on history, applications and modifications of nanomedicine, read Petros and DeSimone, 2010 [25]. endothelial permeability and impaired lymphatic drainage at inflammatory sites by Matsumura and Maeda in 1986, and was called the enhanced permeability and retention (EPR) effect [16]. Oncospar® (polymerdrug conjugate PEG-L-asparaginase) in 1994 and Doxil® (liposomal doxorubicine) in 1995, were the first nanopharmaceuticals to be approved by the American Food and Drug Administration (FDA) [25]. Since then the field of nanomedicine has evolved rapidly, mainly driven by the progress in techniques [18].

\subsubsection{Nanoparticles for drug delivery}

Types of nanoparticles for drug delivery or diagnostics include liposomes, polymeric nanoparticles, micelles, natural nanoparticles like HDL and LDL, nanoemulsions, polymer drug conjugates, protein-drug conjugates, hydrogels and RNA and DNA nanoparticles. New structures and hybrid variants continue to emerge. These nanoparticles are generally used to extend the plasma half-life, improve the therapeutic index and reduce immune response. Properties of nanoparticles, such as size, surface characteristics and shape, play a key role in their biodistribution. [25]

The size of a nanoparticle is very important to control its fate. A diameter of less than $5 \mathrm{~nm}$ results in rapid renal clearance or extravasation from circulation. However, once larger than $1 \mu \mathrm{m}$, nanoparticles accumulate primarily in the liver, spleen and bone marrow, if even larger, they can be lethal in high dose by occluding capillaries. Spherical nanoparticles with a diameter between 10 and $100 \mathrm{~nm}$ have the ability to take advantage of the EPR effect for passive targeting of macrophages at inflammatory sites. While nanoparticles between 100 and $200 \mathrm{~nm}$ have the highest potential for prolonged circulation. [25] For these reasons, many nanoparticles have a diameter around $100 \mathrm{~nm}$.

Surface chemistry, like a hydrophilic polyethylene glycol (PEG) coating, reduces the binding of plasma proteins to the particle. This process of opsonization activates the clearance by phagocytic cells. Active targeting to specific cell receptors, can be achieved by attaching matching ligands and the release of the payload can be triggered for example by heat and $\mathrm{pH}$, or tailored using enzymatically degradable materials. [25].

\section{REFERENCES}

[1] Ana L. Alessandri et al. "Resolution of inflammation: Mechanisms and opportunity for drug development." In: Pharmacology and Therapeutics 139.2 (2013), pp. 189-212. 
[2] “All natural." In: Natural chemical biology 3.7 (2007), p. 351.

[3] P. Allavena and A. Mantovani. "Immunology in the clinic review series; focus on cancer: tumour-associated macrophages: undisputed stars of the inflammatory tumour microenvironment." In: Clinical and experimental immunology 167.2 (Feb. 2012), pp. 195205.

[4] Toru Atsumi et al. "Inflammation amplifier, a new paradigm in cancer biology." In: Cancer research 74.1 (Jan. 2014), pp. 8-14.

[5] Christopher D. Buckley, Derek W. Gilroy, and Charles N. Serhan. "Proresolving lipid mediators and mechanisms in the resolution of acute inflammation." In: Immunity 40.3 (2014), pp. 315-327.

[6] Philippe Chanson and Sylvie Salenave. "Metabolic Syndrome in Cushing's Syndrome." In: Neuroendocrinology 92.1 (2010), pp. 96101.

[7] Richard P. Feynman. "There's plenty of room at the bottom." In: Engineering and Science (Caltech) 23.5 (1960), pp. 22-36.

[8] Raju Gautam and Sanjay M. Jachak. "Recent developments in anti-inflammatory natural products." In: Medicinal research reviews 29.5 (Sept. 2009), pp. 767-820.

[9] Anke Hartmann. "Back to the roots - dermatology in ancient Egyptian medicine." In: JDDG: Journal der Deutschen Dermatologischen Gesellschaft 14.4 (Apr. 2016), pp. 389-396.

[10] Paul L. Huang. "A comprehensive definition for metabolic syndrome." In: Disease models E mechanisms 2.5-6 (2009), pp. 231-237.

[11] Inflammation. May 2016.

[12] Kewal K. Jain. "Nanomedicine: Application of Nanobiotechnology in Medical Practice." In: Medical Principles and Practice 17.2 (2008), pp. 89-101.

[13] Jaspinder Kaur. "A comprehensive review on metabolic syndrome." In: Cardiology Research and Practice 2014 (2014).

[14] Aldons J. Lusis, Alan D. Attie, and Karen Reue. "Metabolic syndrome: from epidemiology to systems biology." In: Nature reviews. Genetics 9.11 (2008), pp. 819-30.

[15] David P. Macfarlane, Shareen Forbes, and Brian R. Walker. "Glucocorticoids and fatty acid metabolism in humans: Fuelling fat redistribution in the metabolic syndrome." In: Journal of Endocrinology 197.2 (2008), pp. 189-204.

[16] Y. Matsumura and $\mathrm{H}$ Maeda. "A new concept for macromolecular therapeutics in cancer chemotherapy: mechanism of tumoritropic accumulation of proteins and the antitumor agent smancs." In: Cancer research 46.12 Pt 1 (Dec. 1986), pp. 6387-92. 
[17] Ruslan Medzhitov. "Origin and physiological roles of inflammation." In: Nature 454.7203 (July 2008), pp. 428-35.

[18] Krukemeyer M.G., Krenn V., and Huebner F. "History and Possible Uses of Nanomedicine Based on Nanoparticles and Nanotechnological Progress." In: Journal of Nanomedicine E Nanotechnology 06.06 (2015).

[19] Andreas G. Moraitis et al. "The role of glucocorticoid receptors in metabolic syndrome and psychiatric illness." In: The Journal of steroid biochemistry and molecular biology 2015 (Mar. 2016).

[20] "Natural products: a continuing source of novel drug leads." In: Biochimica et biophysica acta 1830.6 (June 2013), pp. 3670-95.

[21] S. O'Neill and L. O'Driscoll. "Metabolic syndrome: A closer look at the growing epidemic and its associated pathologies." In: Obesity Reviews 16.1 (2015), pp. 1-12.

[22] World Health Organization. Traditional and Complementary Medicine policy. 2012, pp. 1-17.

[23] Ian Paterson and Edward a Anderson. "Chemistry. The renaissance of natural products as drug candidates." In: Science 310.5747 (Oct. 2005), pp. 451-453.

[24] Peter Libby. "Inflammatory mechanisms: the molecular basis of inflammation and disease." In: Nutrition reviews 65.12 Pt 2 (Dec. 2007), S140-6.

[25] Robby A. Petros and Joseph M. DeSimone. "Strategies in the design of nanoparticles for therapeutic applications." In: Nature reviews. Drug discovery 9.8 (Aug. 2010), pp. 615-27.

[26] Stéphane Quideau et al. "Plant polyphenols: chemical properties, biological activities, and synthesis." In: Angewandte Chemie (International ed. in English) 50.3 (Jan. 2011), pp. 586-621.

[27] Tuhinadri Sen and Samir Kumar Samanta. "Medicinal Plants, Human Health and Biodiversity: A Broad Review." In: Advances in biochemical engineering/biotechnology 147 (July 2014), pp. 59-110.

[28] J Lauran Stöger, Pieter Goossens, and Menno P J de Winther. "Macrophage heterogeneity: relevance and functional implications in atherosclerosis." In: Current vascular pharmacology 8.2 (2010), pp. 233-48.

[29] Christy C. Tangney and Heather E. Rasmussen. "Polyphenols, inflammation, and cardiovascular disease." In: Current atherosclerosis reports 15.5 (May 2013), p. 324. 


\section{SYSTEMATIC REVIEW}

\section{Anti-Inflammatory Properties of Natural Products - a Systematic Review ${ }^{1}$}

Iris E. Allijn, René P. Brinkhuis, Gert Storm, Raymond M. Schiffelers.

\section{ABSTRACT}

Traditionally, natural medicines have been administered as plant extracts, which are composed of a mixture of molecules. The individual molecular species in this mixture may or may not contribute to the overall medicinal effects and some may even oppose the beneficial activity of others. To better control therapeutic effects, studies that characterize specific molecules and describe their individual activity have been performed over the past decades. These studies appear to underline that natural products are particularly effective as anti-oxidants and anti-inflammatory agents. In this systematic review we aimed to identify potent anti-inflammatory natural products and relate their efficacy to their chemical structure and physicochemical properties. To identify these compounds, we performed a comprehensive literature search to find those studies, in which a dose-response description and a positive control reference compound was used to benchmark the observed activity. Of the analyzed papers, $7 \%$ of initially selected studies met these requirements and were subjected to further analysis. This analysis revealed that most selected natural products indeed appeared to possess anti-inflammatory activities, in particular anti-oxidative properties. In addition, $14 \%$ of the natural products outperformed the remaining natural products in all tested assays and are attractive candidates as new anti-inflammatory agents.

\subsection{INTRODUCTION}

Traditional medicines have been used for thousands of years [6o]. Generally they are composed of plants or their extracts. Their continuing

1 Manuscript submitted 
popularity is indicated by the increasing number of publications in scientific biomedical literature databases, like Pubmed [55].

These traditional medicines are composed of a mixture of molecules. The individual molecular species in this mixture may or may not contribute to their medicinal effects and some may even oppose their beneficial activity. This makes interpretation of the therapeutic outcome difficult as the exact composition of and ratio between the molecular species is unknown. The composition and ratio may be affected by various factors like growth conditions, harvesting methods and extraction procedures, which makes comparison and analysis of therapeutic effects challenging.

To better control the therapeutic effects, characterization of the molecular species within extracts and description of their individual activity has been given emphasis over the past decades [26]. The picture that emerges is that natural products appear particularly effective antioxidant and anti-inflammatory agents $[67,30,64]$. However, given the large number of pathways involved in inflammation and lack of standardization in inflammation assays, considerable variation exists in experimental conditions and reported outcomes. This makes the selection of specific natural products with potency for distinct inflammatory pathways still demanding.

The aim of this study is to identify potent anti-inflammatory natural products and to relate their activity to their physicochemical characteristics and chemical structure. To allow the assessment of the relative anti-inflammatory potency, we included only studies that addressed a dose response relationship and the comparison with a positive control compound. This selection formed the basis for the systematic review presented here.

\subsection{ARTICLE SELECTION}

Two large scientific databases (Web of Knowledge [66] and Pubmed [55]) have been used to find original research articles. In the first crude search, articles were filtered on topic (natural products, natural compounds, inflammation, in vitro, in vivo), document type (primary article), and language (English). This initial search yielded 653 different primary articles.

These articles were further filtered to obtain single plant-sourced molecular species, excluding extracts and mixtures. Finally, for all papers meeting these requirements, the experimental setup was addressed. We required a clear description of cellular or animal model systems used, inclusion of controls, inclusion of a positive control reference 
compound and dose response studies (the entire article selection flow and requirements are presented in Figure 2.1).

The final article selection comprises 45 articles, describing 102 different natural products, 31 different reference compounds and 25 comparable anti-inflammatory tests (Tables 2.1 and 2.2). This research originates mainly from Asia (52\%), followed by Europe (22\%), Africa (12\%), South America (9\%) and North America (5\%) and covers the period 2000 to 2013.

\subsection{STRUCTURAL CLASSIFICATION}

The natural products (1-102) and the reference compounds (103-133) from the selected articles, were divided in eight defined structural classes and a miscellaneous group: coumarins, catechol derivatives, porphyrins, sterols, diterpenoids, stilbenes, flavonoids and rest. Natural products containing one or more sugar moieties were placed in the group of the corresponding chemical backbone. The group sizes were as follows: coumarins $=$ flavonoids $>$ rest $>$ sterols $>$ catechol derivatives $>$ diterpenoids $>$ porphyrins $>$ stilbenes.

\subsubsection{Coumarins}

Coumarins are under investigation for their anti-inflammatory, antimicrobi-al and anti-fungal properties [12]. In this analysis, the coumarin group consists of twenty natural products (1-20) (Table 2.3, Figure 2.2). Two compounds, siphonochilone (5) and methyl breviolincarboxylate (16) are strictly speaking not coumarins, but do share important structural elements with this class. At the same time, their distribution coefficients $(\log \mathrm{D}$ at $\mathrm{pH} 7.4)$ do represent the extremes within this group. Fraxetin (20) is exclusively used as a positive control reference compound, however, since this compound is from plant origin, it is placed with the natural products. The coumarin group is fairly homogeneous in physicochemical characteristics with a median molecular weight (MW) of 258.25 (186.17-334.32) and a $\log \mathrm{D}$ at $\mathrm{pH} 7.4$ of 1.78 .

\subsubsection{Catechol derivatives}

Catechol groups have strong anti-oxidant effects, and the main function of these structures in plants is anti-oxidant activity. The catechol derivatives group consists of thirteen natural products (21-33) (Table 2.4, Figure 2.3) and three reference compounds (103-105) (Table 2.5, Figure 2.4). The natural product pyrogallol (33) is solely used as a reference compound. From the reference compounds, $\alpha$-tocopherol (104) 


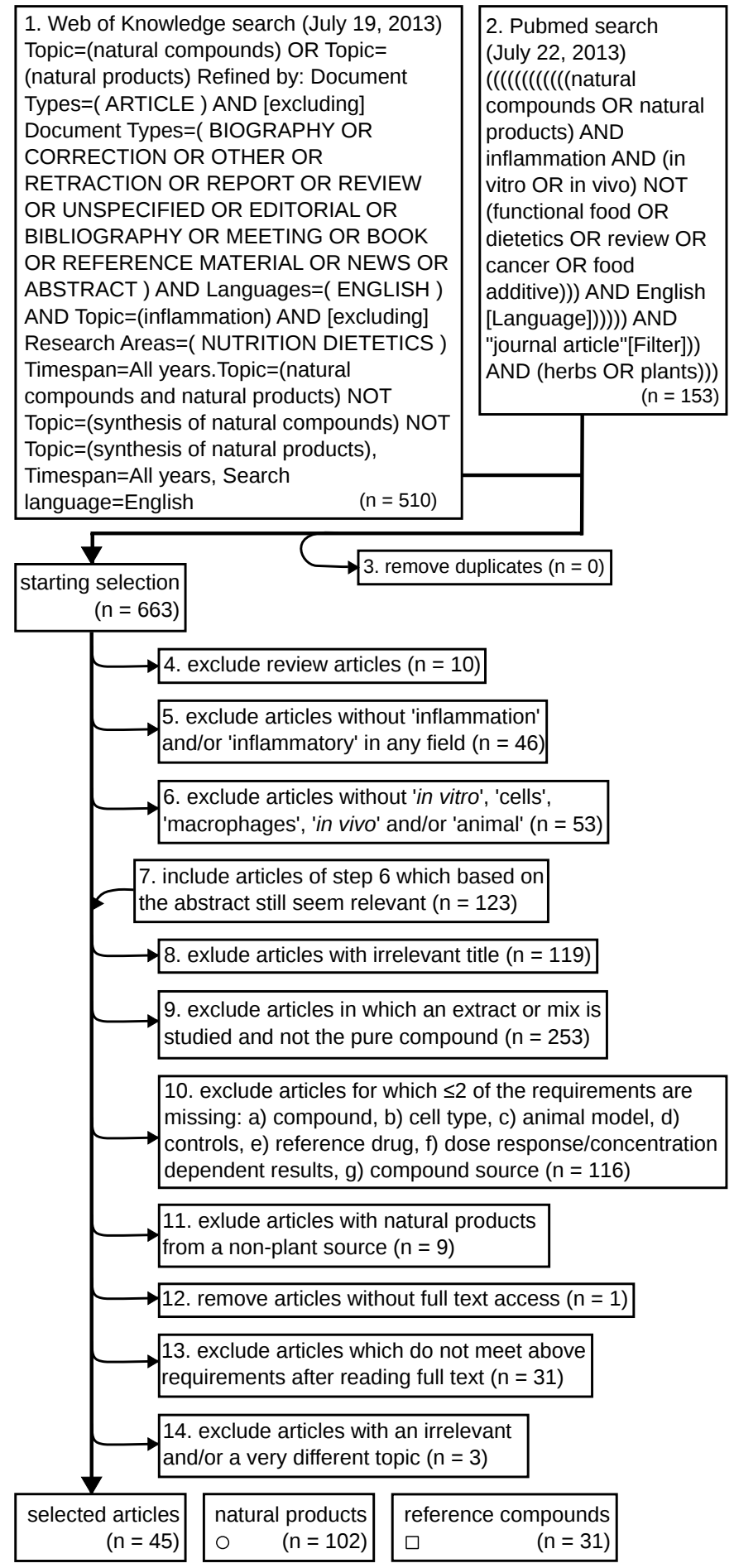

Figure 2.1: Article selection flow diagram. Article search resulted in a final selection of 45 articles containing 102 plant-sourced natural products, of which 6 were also used as reference compound, and 31 synthetic reference compounds. 
Table 2.1: Content of selected articles A - H. Only the 25 comparable assays are displayed (Tables $\mathrm{S}_{3}-\mathrm{S}_{7}$ ), additional assay list can be found in

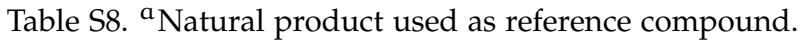

\begin{tabular}{|c|c|c|}
\hline First author, year & Natural \& Reference & Anti-inflamamtory assays \\
\hline Abdelwahab, $2011[1]$ & $47 \& 114,125,131,133$ & NO, COX-2, COX-1, r_paw \\
\hline Ashalatha, 2010 [3] & $48,81 \&-$ & $N F-k \beta$ \\
\hline Ban, 2009 [4] & $84 \& 125$ & NO, iNOS, COX-2, m_ear, r_paw \\
\hline Bao, 2009 [5] & $30,31,73,75,76,79,82 \& 108$ & his, m_paw \\
\hline Baolin, 2004 [6] & $61,71 \& 71^{a}$ & TNF- $\alpha, \mathrm{LTB}_{4}, \mathrm{PDG}_{2}$, his \\
\hline Bas, $2007[7]$ & $91 \& 108,120$ & NO, iNOS, TNF- $\alpha$, IL-1 $\beta$, IL-2, LTB 4 , m_ear \\
\hline Bose, $2011[8]$ & $2 \& 108$ & IL-6, TNF- $\alpha$ \\
\hline Capasso, 2008 [11] & $102 \&-$ & only an incomparable assay used \\
\hline \multirow{2}{*}{ Chandrasekaran, 2011 [12] } & $54,55,56,58,66,68 \&$ & NO, IL-6, TNF- $\alpha$, IL-1 $\beta$, IL-2 \\
\hline & $108,112,113,114,129$ & $\mathrm{PGE}_{2}, \mathrm{LTB}_{4}, \mathrm{TXB}_{2}$, his \\
\hline Chao, 2005 [13] & $39,10 \& 100^{a}, 123$ & DPPH, TNF- $\alpha$, IL-1 $\beta$ \\
\hline Chen, 2010 [14] & $71 \& 107$ & IL-6, TNF- $\alpha$ \\
\hline Chi, $2003[15]$ & $65 \& 107$ & mear \\
\hline Chung, 2003 [16] & $61,62,63 \& 110,125$ & NO, DPPH, LPO, iNOS, $\mathrm{PGE}_{2}$, r_paw \\
\hline Corea, $2005[17]$ & $50 \& 108$ & NO, iNOS, TNF- $\alpha$, NF- $k \beta, \mathrm{PGE}_{2}, \mathrm{COX}-2, \mathrm{r} \_$paw \\
\hline Dugasani, $2010[21]$ & $22,23,24,25 \& 104,111$ & $\mathrm{NO}, \mathrm{DPPH}, \mathrm{O}_{2}, \mathrm{OH}, \mathrm{PGE}_{2}$ \\
\hline Enomoto, 2007 [22] & $65 \& 108$ & r_paw, PGE 2 \\
\hline \multirow[t]{2}{*}{ Fang, 2008 [23] } & & NO, DPPH, IL-6, TNF- $\alpha$ \\
\hline & $87,95,99 \& 104$ & \\
\hline Fernandez-Arche, 2010 [24] & $44 \& 108,125$ & $\mathrm{NO}, \mathrm{PGE}_{2}, \mathrm{~m} \_$ear \\
\hline Fouche, 2011 [25] & $5 \& 103,121$ & his \\
\hline Gao, 2009 [29] & $49 \& 106$ & NO, IL-6, TNF- $\alpha$ \\
\hline Guo, 2008 [32] & $97 \& 108,109,125,127$ & $\mathrm{NO}, \mathrm{iNOS}, \mathrm{NF}-\kappa \beta, \mathrm{PGE}_{2}, \mathrm{COX}-2$ \\
\hline Hernández, 2005 [34] & $60 \& 108,120,125$ & NO, $\mathrm{LTB}_{4}, \mathrm{COX}-2, \mathrm{COX}-1$, el, m_ear, m_paw \\
\hline Hou, 2010 [35] & $77 \& 118,133$ & NO, ROS \\
\hline
\end{tabular}


Table 2.2: Content of selected articles I - Z. Only the 25 comparable assays are displayed (Tables $\mathrm{S}_{3}-\mathrm{S}_{7}$ ), additional assay list can be found in Table S8. ${ }^{a}$ Natural product used as reference compound, ${ }^{b}$ Unit of measurement adjusted for comparison.

\begin{tabular}{|c|c|c|}
\hline First author, year & Natural \& Reference & Anti-inflamamtory assays \\
\hline Jan, 2010 [37] & $92,93,94 \&-$ & IL-6, TNF- $\alpha$ \\
\hline Kenny, 2013 [41] & $46,51,52 \& 128,132$ & NO, IL-2, IL-8 \\
\hline Khan I., 2011 [42] & $41,83 \& 83^{a}, 124$ & r_paw \\
\hline Khan S., 2013 [43] & $74 \& 108,109,117,127$ & $\mathrm{NO}, \mathrm{iNOS}, \mathrm{TNF}-\alpha, \mathrm{PGE}_{2}, \mathrm{COX}-2, \mathrm{~m} \_$paw \\
\hline Kourounakis, 2002 [45] & 85,86 \& 119, 125 & LPO, m_paw \\
\hline Lu, 2012 [47] & $40 \& 107$ & only incomparable assays used \\
\hline Mengoni, 2011 [48] & $38,39 \& 125$ & NO, m_ear ${ }^{b}$ \\
\hline Mnonopi, 2011 [49] & $57 \& 114,116$ & $\mathrm{TXB}_{2}$ \\
\hline Muhammad, 2003 [51] & $26,27,28 \& 115,126$ & ROS \\
\hline Nardi, 2007 [53] & $72 \& 125$ & only incomparable assays used \\
\hline Njamen, 2003 [56] & $46,51,52 \& 70^{a}, 108,125,130$ & DPPH, $\mathrm{LTB}_{4}, \mathrm{COX}-1, \mathrm{~m} \_$ear, m_paw \\
\hline Oh, $2011[57]$ & $100 \&-$ & $N F-k \beta$ \\
\hline Okoye, 2010 [58] & $42,43,45 \& 107,125$ & m_ear, r_paw \\
\hline Olajide, 2009 [59] & $96 \& 125$ & r_paw \\
\hline Ospina, $2001[61]$ & $20,21 \& 20^{a}, 105,125$ & $\mathrm{O}_{2}, \mathrm{LPO}, \mathrm{el}, \mathrm{PGE}_{2}$ \\
\hline Ren, 2007 [65] & $29 \& 125$ & $\mathrm{NO}$, iNOS, $\mathrm{PGE}_{2}, \mathrm{COX}-2, \mathrm{r} \_$paw \\
\hline Sala, $2003[70]$ & $33,64,67,78 \& 33^{a}, 108,110,125,130$ & $\mathrm{DPPH}, \mathrm{O}_{2}, \mathrm{LPO}, \mathrm{LTB}_{4}, \mathrm{~m} \_$ear, m_paw \\
\hline Santa-Cecília, 2012 [71] & $101 \& 110,115$ & $\mathrm{DPPH}$ \\
\hline Subramoniam, 2012 [72] & $34,35,36,37,100 \& 100^{a}$ & $\mathrm{O}_{2}, \mathrm{OH}, \mathrm{NF}-\mathrm{K} \beta$ \\
\hline Taha, 2012 [73] & $90 \& 122$ & only incomparable assays used \\
\hline \multirow[t]{2}{*}{ Wang, $2000[75]$} & $1,2,3,4,6,7,8,9,10,11$ & \multirow[t]{2}{*}{$\mathrm{NO}$} \\
\hline & $12,13,14,15,17,18,19 \&-$ & \\
\hline Zhang, 2006 [81] & $88 \& 104$ & m_ear \\
\hline
\end{tabular}


Table 2.3: Coumarins - natural products $(n=20)$.

\begin{tabular}{|c|c|c|c|c|}
\hline Name & CAS number & MW & $\log D(p H 7.4)$ & tPSA \\
\hline Psoralen (1) & $66-97-7$ & 186.17 & 1.94 & 39.44 \\
\hline Bergapten (2) & $484-20-8$ & 216.19 & 1.78 & 48.67 \\
\hline Xanthotoxin (3) & $298-81-7$ & 216.19 & 1.78 & 48.67 \\
\hline Isopimpinellin (4) & $482-27-9$ & 246.22 & 1.63 & 57.90 \\
\hline Siphonochilone (5) & $438041-86-2$ & 230.31 & 3.76 & 30.21 \\
\hline Cnidilin (6) & $14348-22-2$ & 300.31 & 2.99 & 57.90 \\
\hline Isoimperatorin (7) & $482-45-1$ & 270.28 & 3.15 & 48.67 \\
\hline Oxypeucadanin (8) & $737-52-0$ & 286.28 & 2.32 & 61.20 \\
\hline Oxypeucadanin hydrate (9) & $2643-85-8$ & 304.30 & 1.16 & 89.13 \\
\hline Heraclenol (10) & $31575-93-6$ & 304.30 & 1.16 & 89.13 \\
\hline Heraclenin (11) & $2880-49-1$ & 286.28 & 2.32 & 61.20 \\
\hline Phellopterin (12) & $2543-94-4$ & 300.31 & 2.99 & 57.90 \\
\hline Byakangelicol (13) & $26091-79-2$ & 316.31 & 2.17 & 70.43 \\
\hline Byakangelicin (14) & $482-25-7$ & 334.32 & 1.00 & 98.36 \\
\hline Angelicin (15) & $523-50-2$ & 186.17 & 1.94 & 39.44 \\
\hline Methyl breviolincarboxylate (16) & $154702-76-8$ & 306.23 & -0.33 & 130.36 \\
\hline Isobergapten (17) & $482-48-4$ & 216.19 & 1.78 & 48.67 \\
\hline Sphondin (18) & $483-66-9$ & 216.19 & 1.78 & 48.67 \\
\hline Pimpinellin (19) & $131-12-4$ & 246.22 & 1.63 & 57.90 \\
\hline Fraxetin $^{a}(\mathbf{2 0})$ & $574-84-5$ & 208.17 & 1.64 & 75.99 \\
\hline Median values & & 258.25 & 1.78 & 57.90 \\
\hline
\end{tabular}


is approved by the US Food and Drug Administration (FDA) [13] and propyl gallate (105) is a registered, however restricted, food additive by the European Food Safety Authority (EFSA) [14].

The physicochemical properties of this group are primarily determined by the length of the hydrocarbon chain. Longer hydrocarbon chains increase the $M W$ and lipophilicity and hence the $M W$ and $\log D$ at $\mathrm{pH} 7.4$ have quite a wide range with median values of 294.39 (126.11 $-364.53)$ and $3.69(-2.48-5.39)$, respectively.

\subsubsection{Porphyrins}

The porphyrins group consists of four natural products (34-37), there are no reference compounds (Table 2.6, Figure 2.5). This is the group with the largest structures, resulting in the highest MW. Also their typical macrocyclic structure results in the highest LogD values of all compounds in this dataset.

\subsubsection{Sterols}

Plant sterols are both structurally as well as functionally related to cholesterol in animals [8o]. They are an integral part of the cell mem-

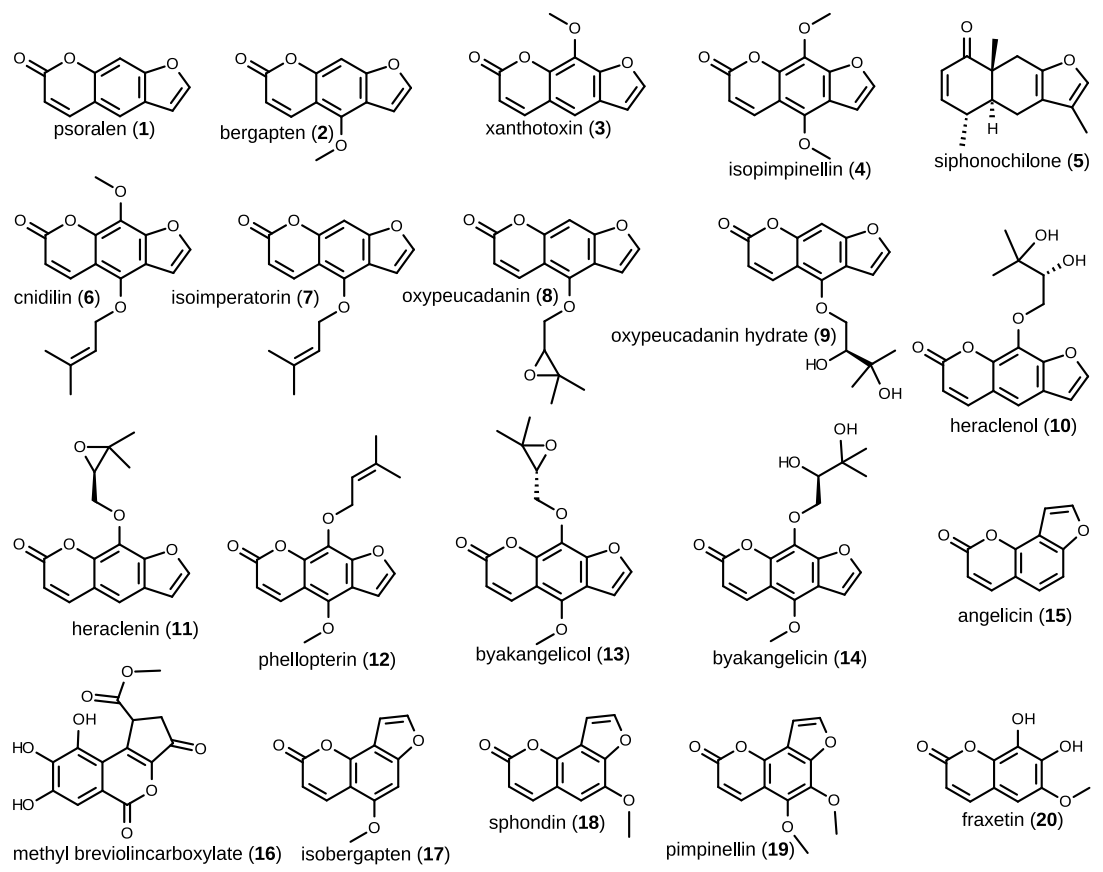

Figure 2.2: Coumarins - natural products. 
Table 2.4: Catechol derivatives - natural products $(n=13)$.

\begin{tabular}{lllll}
\hline Name & CAS RN & MW & $\operatorname{logD}(\mathrm{pH} 7.4)$ & tPSA \\
\hline Rapanone (21) & $573-40-0$ & 322.45 & 3.69 & 74.60 \\
6-gingerol (22) & $23513-14-6$ & 294.39 & 3.62 & 66.76 \\
8-gingerol (23) & $23513-08-8$ & 322.45 & 4.50 & 66.76 \\
10-gingerol (24) & $23513-15-7$ & 350.50 & 5.39 & 66.76 \\
6-shogaol (25) & $555-66-8$ & 267.38 & 4.84 & 46.53 \\
Maesanol (26) & $156979-72-5$ & 362.51 & 4.56 & 74.60 \\
Maesanin (27) & $82380-21-0$ & 362.51 & 4.56 & 63.60 \\
Dihydromaesanin (28) & $21551-64-4$ & 364.53 & 5.01 & 63.60 \\
Linolenic acid (29) & $463-40-1$ & 278.44 & 3.68 & 37.30 \\
Gallic acid (30) & $149-91-7$ & 170.12 & -2.48 & 97.99 \\
Methyl gallate (31) & $99-24-1$ & 184.15 & 0.99 & 86.99 \\
Ethyl gallate (32) & $831-61-8$ & 198.17 & 1.35 & 86.99 \\
Pyrogallol ${ }^{a}$ (33) & $87-66-1$ & 126.11 & 1.05 & 60.69 \\
\hline Median values & & 294.39 & 3.69 & 66.76 \\
\hline
\end{tabular}

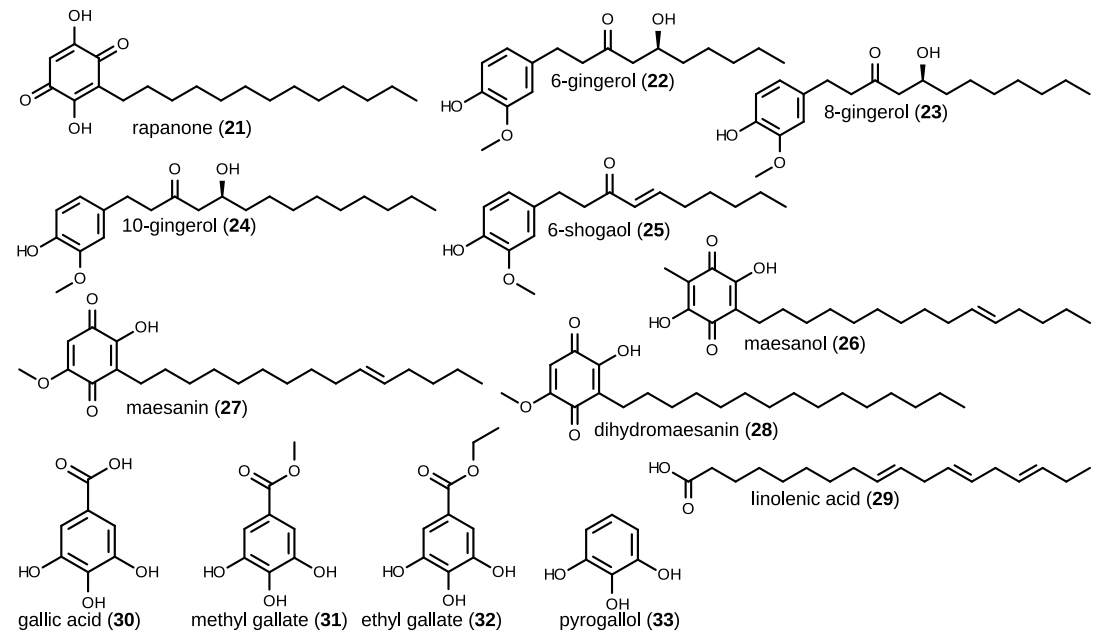

Figure 2.3: Catechol derivatives - natural products. 
Table 2.5: Catechol derivatives - reference compounds $(n=3)$.

\begin{tabular}{lllll}
\hline Name & CAS RN & MW & $\log \mathrm{D}(\mathrm{pH} 7.4)$ & tPSA \\
\hline RO 20-1724 (103) & $29925-17-5$ & 278.35 & 1.99 & 59.59 \\
-tocopherol $^{\mathrm{b}}$ (104) & $50-02-9$ & 430.72 & 10.51 & 29.46 \\
Propyl gallate $^{\mathrm{c}}$ (105) & $121-79-9$ & 212.20 & 1.87 & 86.99 \\
\hline Median values & & 278.35 & 1.99 & 59.59 \\
\hline
\end{tabular}
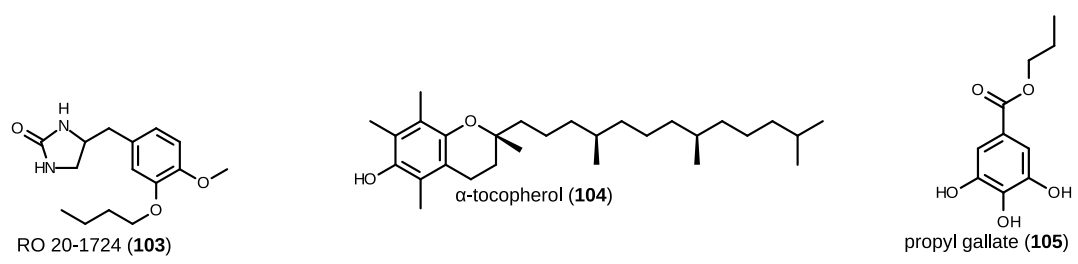

Figure 2.4: Catechol derivatives - reference compounds.

Table 2.6: Porphyrins - natural products $(n=4)$.

\begin{tabular}{lllll}
\hline Name & CAS RN & MW & $\log$ ( $\mathrm{pH} \mathrm{7.4)}$ & tPSA \\
\hline Chlorophyll A (34) & $479-61-8$ & 893.51 & 12.40 & 121.23 \\
Chlorophyll B (35) & $519-62-0$ & 907.49 & 11.60 & 138.30 \\
Pheophytin A (36) & $603-17-8$ & 871.22 & 12.48 & 127.03 \\
Pheophytin B (37) & $3147-18-0$ & 885.20 & 11.67 & 144.10 \\
\hline Median values & & 889.36 & 12.04 & 132.67 \\
\hline
\end{tabular}


brane and are important regulators of membrane fluidity and permeability and act as signal transductors.

The sterols structural group has sixteen natural products (38-53) (Table 2.7, Figure 2.6) and three reference compounds (106-108) (Table 2.8, Figure 2.7). Three natural products in this group, $\alpha$-chaconine $(5 \mathbf{1}), \alpha-$ solanine (52) and daucosterol (53) carry one or more sugar moieties.

The reference compounds in this group are the widely known, robust and FDA approved [13] corticosteroids hydrocortisone (106), prednisolone (107) and dexamethasone (108). Dexamethasone (108) is an often used reference compound in this systematic review, featuring in eleven studies.

The sterols have strongly variably $\log \mathrm{D}$ values at $\mathrm{pH} 7.4$ which are determined by the molecules' side groups. Sugar moieties confer a strong hydrophilic character to the molecules whereas (branched) hydrocarbon side chains make the molecule very hydrophobic.

\subsubsection{Diterpenoids}

Diterpenoids are a subgroup of the terpenoids, the most abundant type of natural products. Their main function in plants appears to be repellents for bugs and insects [31]. This diterpenoids group has seven natural products (54-60) (Table 2.9, Figure 2.8), none of the reference compounds fall within this group (Table 2.9, Figure 2.8). All but dehydrocostic acid (60) are bicyclic diterpenoids. Neoandrographolide (59) carries a sugar moiety, which explains why it has an aberrant MW.

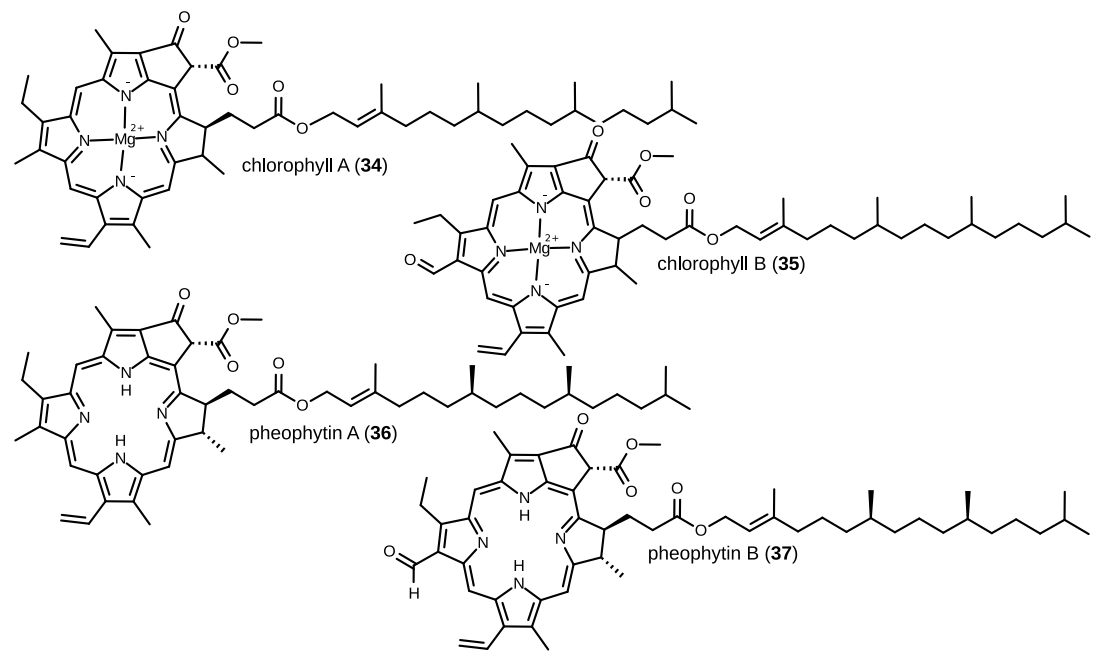

Figure 2.5: Porphyrins - natural products. 
Table 2.7: Sterols - natural products $(n=16)$.

\begin{tabular}{|c|c|c|c|c|}
\hline Name & CAS RN & MW & $\log D(p H 7.4)$ & tPSA \\
\hline Carnosic acid ( 38$)$ & $3650-09-7$ & 332.44 & 2.17 & 77.76 \\
\hline Sugiol (39) & $511-05-7$ & 300.44 & 5.04 & 37.30 \\
\hline Kirenol $^{a}(40)$ & $52659-56-0$ & 338.49 & 1.16 & 80.92 \\
\hline Taxusabietane A (41) & $220785-02-4$ & 344.45 & 4.28 & 63.60 \\
\hline $5^{\alpha}$-stigmast-23-ene-3,6-dione (42) & $1274717-13-3$ & 426.69 & 3.17 & 34.14 \\
\hline $5 \alpha$-stigmastane-3,6-dione (43) & $22149-69-5$ & 428.70 & 7.53 & 34.14 \\
\hline Tirucallol (44) & $514-46-5$ & 426.73 & 7.71 & 20.23 \\
\hline Pepostanol (45) & $134887-29-9$ & 414.72 & 7.80 & 20.23 \\
\hline Solanidine (46) & $80-78-4$ & 397.65 & 1.39 & 23.47 \\
\hline Cucurbitacin E (47) & $18444-66-1$ & 556.70 & 3.47 & 138.20 \\
\hline Lupeol acetate (48) & $1617-68-1$ & 468.77 & 7.89 & 26.30 \\
\hline Hederagenin (49) & $465-99-6$ & 472.71 & 2.71 & 77.76 \\
\hline Pepluanone (50) & $869959-12-6$ & 656.73 & 2.83 & 168.80 \\
\hline$\alpha$-chaconine (51) & $20562-03-2$ & 852.07 & -1.83 & 220.46 \\
\hline$\alpha$-solanine (52) & $20562-02-1$ & 868.07 & -2.87 & 240.69 \\
\hline Daucosterol (53) & $474-58-8$ & 576.86 & 6.07 & 99.38 \\
\hline Median values & & 491.39 & 3.91 & 85.21 \\
\hline
\end{tabular}

Table 2.8: Sterols - reference compounds $(n=3)$.

\begin{tabular}{lllll}
\hline Name & CAS RN & MW & $\log$ ( $\mathrm{pH} \mathrm{7.4)}^{\text {tPSA }}$ \\
\hline Hydrocortisone $^{\mathrm{b}}(\mathbf{1 0 6})$ & $50-23-7$ & 362.47 & 1.28 & 94.83 \\
Prednisolone $^{\mathrm{b}}(\mathbf{1 0 7})$ & $50-24-8$ & 360.45 & 1.27 & 94.83 \\
Dexamethasone $^{\mathrm{b}}(\mathbf{1 0 8})$ & $50-02-2$ & 392.47 & 1.68 & 94.83 \\
\hline Median values & & 362.47 & 1.28 & 94.83 \\
\hline
\end{tabular}



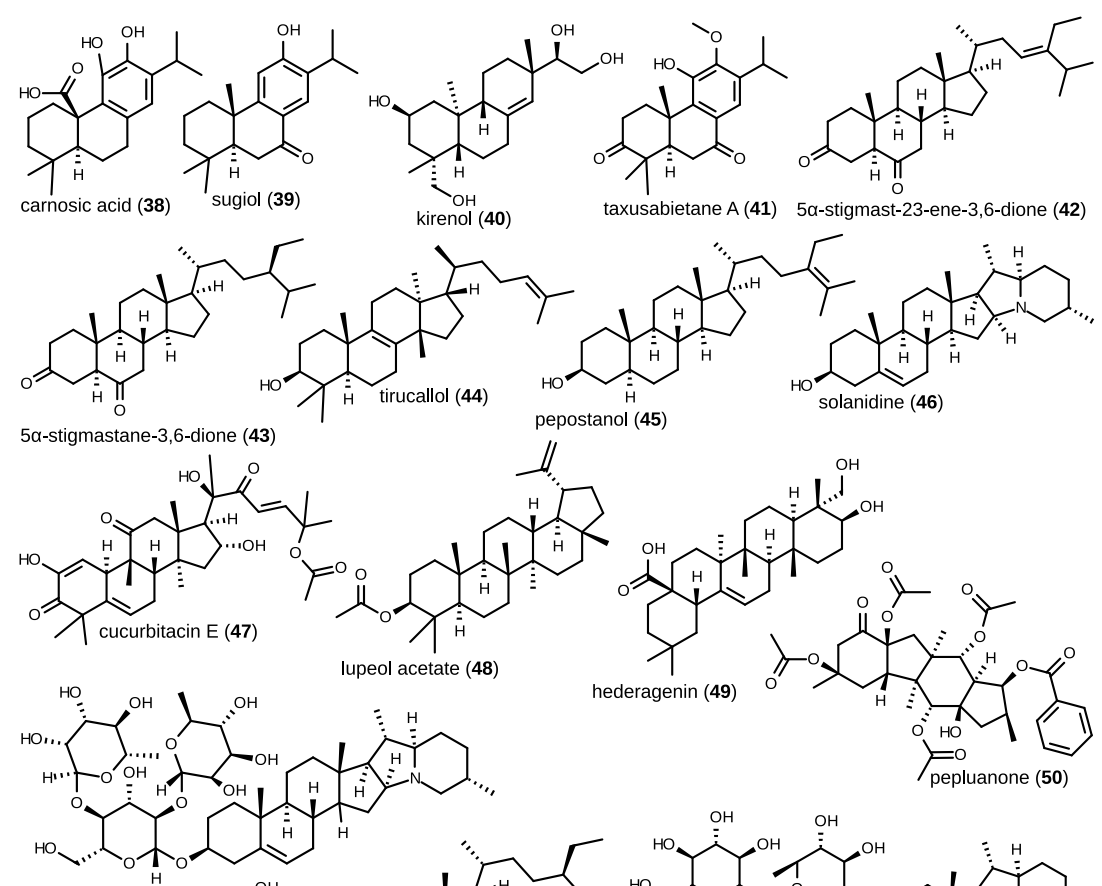

a-chaconine (51)
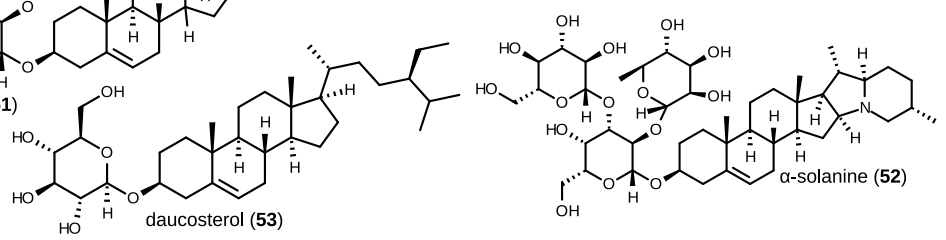

Figure 2.6: Sterols - natural products.
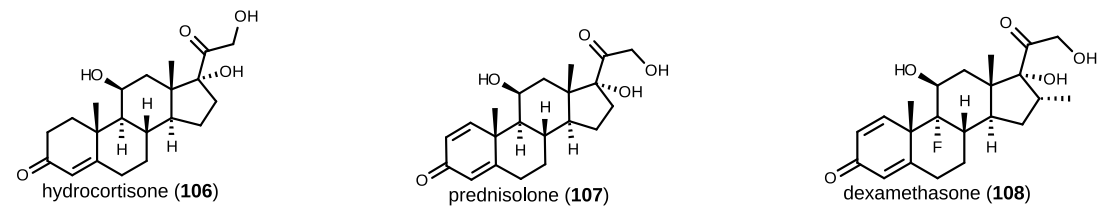

Figure 2.7: Sterols - reference compounds.

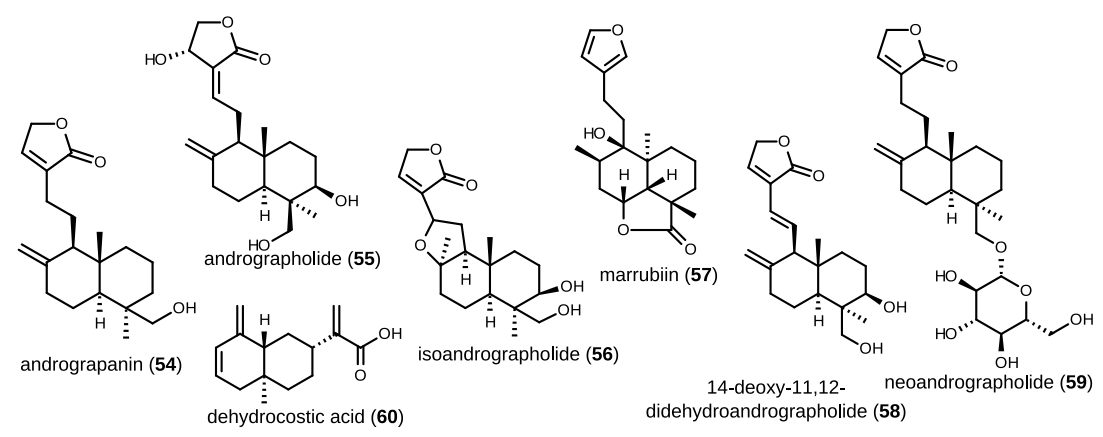

Figure 2.8: Diterpenoids - natural products. 


\subsubsection{Stilbenes}

Stilbenes are considered phytoalexins and are mainly present in grapes [69]. This group is the smallest in this systematic review, consisting of only three natural products (61-63) (Table 2.10, Figure 2.9). Mulberroside A (63) has a sugar moiety at both ends of the molecule, significantly lowering its $\log \mathrm{D}$ value at $\mathrm{pH} 7.4$ compared to the other compounds in this group.<smiles>Oc1ccc(C=Cc2cc(O)cc(O)c2)cc1</smiles>

resveratrol (61)
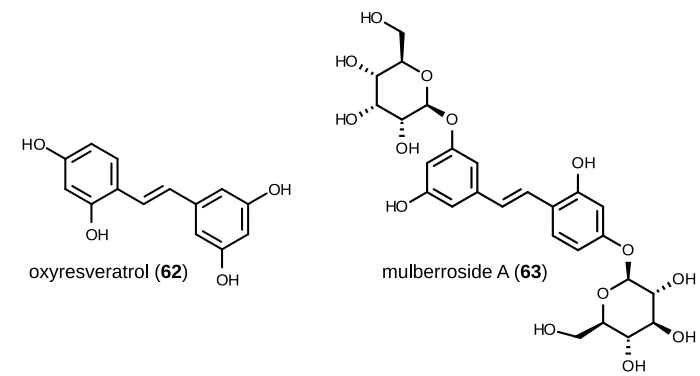

Figure 2.9: Stilbenes - natural products

Table 2.9: Diterpenoids - natural products $(n=7)$.

\begin{tabular}{lllll}
\hline Name & CAS RN & MW & logD (pH 7.4) & tPSA \\
\hline Andrograpanin (54) & $82209-74-3$ & 318.46 & 3.97 & 46.53 \\
Andrographolide (55) & $55208-58-7$ & 350.46 & 1.66 & 86.99 \\
Isoandrographolide (56) & $4176-96-9$ & 350.46 & 1.70 & 75.99 \\
Marrubiin (57) & $465-92-9$ & 332.44 & 3.67 & 59.67 \\
14-Deoxy-11,12-didehydroandrographolide (58) & $42895-58-9$ & 332.44 & 2.38 & 66.76 \\
Neoandrographolide (59) & $27215-14-1$ & 480.60 & 2.20 & 125.68 \\
Dehydrocostic acid (60) & $100108-70-1$ & 232.32 & 1.04 & 37.30 \\
\hline Median values & & 332.44 & 2.20 & 66.76 \\
\hline
\end{tabular}

Table 2.10: Stilbenes - natural products $(n=3)$.

\begin{tabular}{lllll}
\hline Name & CAS RN & MW & $\operatorname{logD}(\mathrm{pH} 7.4)$ & tPSA \\
\hline Resveratrol (61) & $501-36-0$ & 228.25 & 3.37 & 60.69 \\
Oxyresveratrol (62) & $29700-22-9$ & 244.25 & 3.06 & 80.92 \\
Mulberroside A (63) & $102841-42-9$ & 586.53 & -1.46 & 239.22 \\
\hline Median values & & 244.25 & 3.06 & 80.92 \\
\hline
\end{tabular}




\subsubsection{Flavonoids}

Flavonoids are responsible for the vivid colors in leaves, flowers and fruits and play a role in pollination. Furthermore, their bitter taste plays a role in the plant's defense system [63]. The group representing the flavonoids is large, consisting of twenty natural products $(\mathbf{6 4 - 8 3})$ (Table 2.11, Figure 2.10). Quercetin (70) and luteolin (71) are also used as reference and baicalein (83) solely as reference (Table 2.11, Figure 2.10). Five members of this structural group, $(78,79,80,81$ and 82$)$ carry at least one sugar moiety, causing these compounds to have the highest water solubility in this group.

Table 2.11: Flavonoids - natural products $(\mathbf{n}=\mathbf{2 0}) .{ }^{a}$ Natural product also used as reference compound, ${ }^{b}$ Natural product only used as reference compound.

\begin{tabular}{|c|c|c|c|c|}
\hline Name & CAS RN & MW & $\log \mathrm{D}(\mathrm{pH} 7.4)$ & tPSA \\
\hline Pinocembrin (64) & $480-39-7$ & 256.26 & 3.01 & 66.76 \\
\hline Wogonin (65) & $632-85-9$ & 284.27 & 2.37 & 75.99 \\
\hline 7-o-methylwogonin (66) & $3570-62-5$ & 298.29 & 2.88 & 64.99 \\
\hline Gnaphaliin (67) & $33803-42-8$ & 314.29 & 2.09 & 85.22 \\
\hline Skullcapflavone I (68) & $41060-16-6$ & 314.29 & 2.52 & 85.22 \\
\hline Rhamnocitrin (69) & $569-92-6$ & 300.27 & 2.15 & 96.22 \\
\hline Quercetin $^{\mathrm{a}}(\mathbf{7 0})$ & $117-39-5$ & 302.24 & 1.00 & 127.45 \\
\hline Luteolin $^{a}(\mathbf{7 1})$ & $491-70-3$ & 286.24 & 1.46 & 107.22 \\
\hline Catechin (72) & $154-23-4$ & 290.27 & 1.78 & 110.38 \\
\hline Tricetiflavan (73) & $493-44-7$ & 290.27 & 2.55 & 110.38 \\
\hline Capillarisin (74) & $56365-38-9$ & 316.27 & 2.65 & 105.45 \\
\hline (-)-tetrahydroxyflavan-7-gallate (75) & $889447-89-6$ & 442.38 & 3.51 & 177.14 \\
\hline (-)-epigallocatechin-7-gallate (76) & $96658-18-3$ & 458.38 & 2.44 & 197.37 \\
\hline Silymarin (77) & $65666-07-1$ & 482.44 & 2.47 & 155.14 \\
\hline Tiliroside (78) & $20316-62-5$ & 594.53 & 1.73 & 212.67 \\
\hline Quercitrin (79) & $522-12-3$ & 448.38 & -0.25 & 186.37 \\
\hline Rutin (8o) & $153-18-4$ & 610.52 & -2.02 & 265.52 \\
\hline Choerosphondin (81) & $81202-36-0$ & 434.40 & 0.44 & 166.14 \\
\hline Myritricin (82) & $17912-87-7$ & 464.38 & -0.60 & 206.60 \\
\hline Baicalein $^{\mathrm{b}}(83)$ & $491-67-8$ & 272.26 & 2.74 & 86.99 \\
\hline Median values & & 314.29 & 2.26 & 110.38 \\
\hline
\end{tabular}



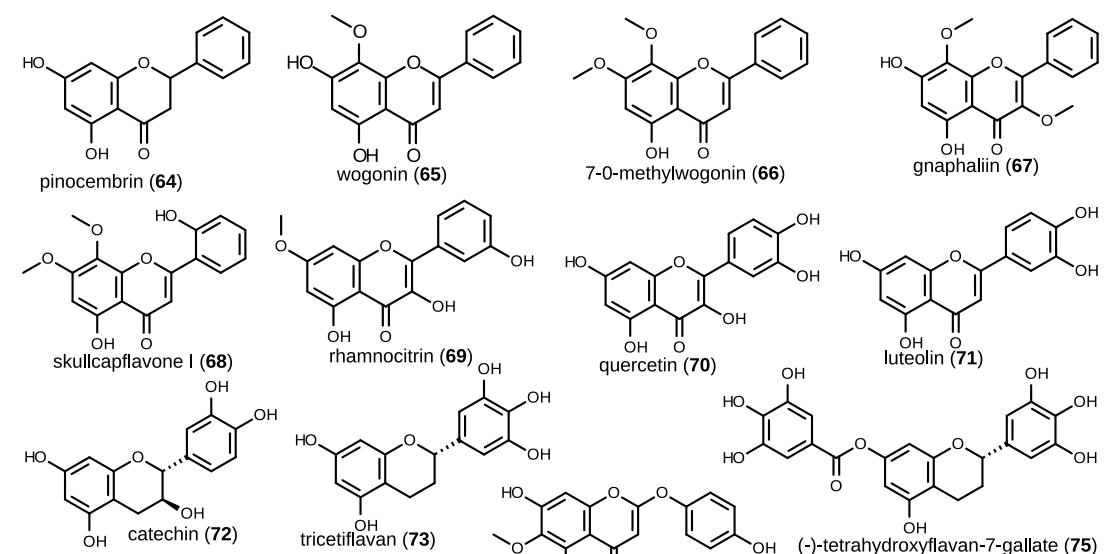<smiles>CC[C@H]1CCc2c(O)cc(O)cc2O1</smiles><smiles>Cc1cccc(I)c1O</smiles>

quercetin (70)<smiles>CCc1cc(O)c(O)c(O)c1</smiles><smiles>C=C(O)C(=C)OC</smiles><smiles>O=C(Oc1cc(O)c2c(c1)O[C@H](c1cc(O)c(O)c(O)c1)[C@H](O)C2)c1cc(O)c(O)c(O)c1</smiles>

(-)-epigallocatechin-7-gallate (76)<smiles>C[C@H]1O[C@H](Oc2c(-c3ccc(O)c(O)c3)oc3cc(O)cc(O)c3c2=O)[C@H](O)[C@@H](O)[C@H]1O</smiles><smiles>O=C1CC(c2ccccc2)Oc2cc(O)c(O)c(O)c21</smiles>

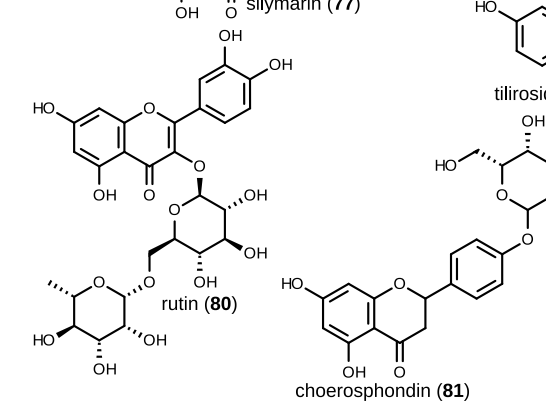
(-)-tetrahydroxyflavan-7-gallate (75) capillarisin (74)<smiles>CCCOCc1ccc(O)c(O)c1</smiles><smiles>C[C@@H]1O[C@H](Oc2c(-c3cc(O)c(O)c(O)c3)oc3cc(O)cc(O)c3c2=O)[C@H](O)[C@@H](O)[C@H](O)[C@H]1O</smiles>

Figure 2.10: Flavonoids - natural products 


\subsubsection{Miscellaneous structures}

This group contains all compounds that do not have structural similarities to any of the above described groups. The rest group contains nineteen different natural products (84-102) (Table 2.12, Figure 2.11) and as much as twenty-five reference compounds (109-133) (Table 2.13, Figure ??). Three natural products do have a sugar moiety: arbutin (9o), scrovalentinoside (91) and sesaminol triglucoside (92), none of the reference compounds have one. Cannabidiol (102) represents the active compound in an approved drug (marketed as Sativex) [10], however the compound has not been used as general reference compound. Indomethacin (125), a very well known nonsteroidal anti-inflammatory drug (NSAID), is the most used reference compound in this entire systematic review, featuring in thirteen studies. Because this group has a high diversity in structures, the $\mathrm{MW}$ and $\log \mathrm{D}$ at $\mathrm{pH} 7.4$ have a very wide range.

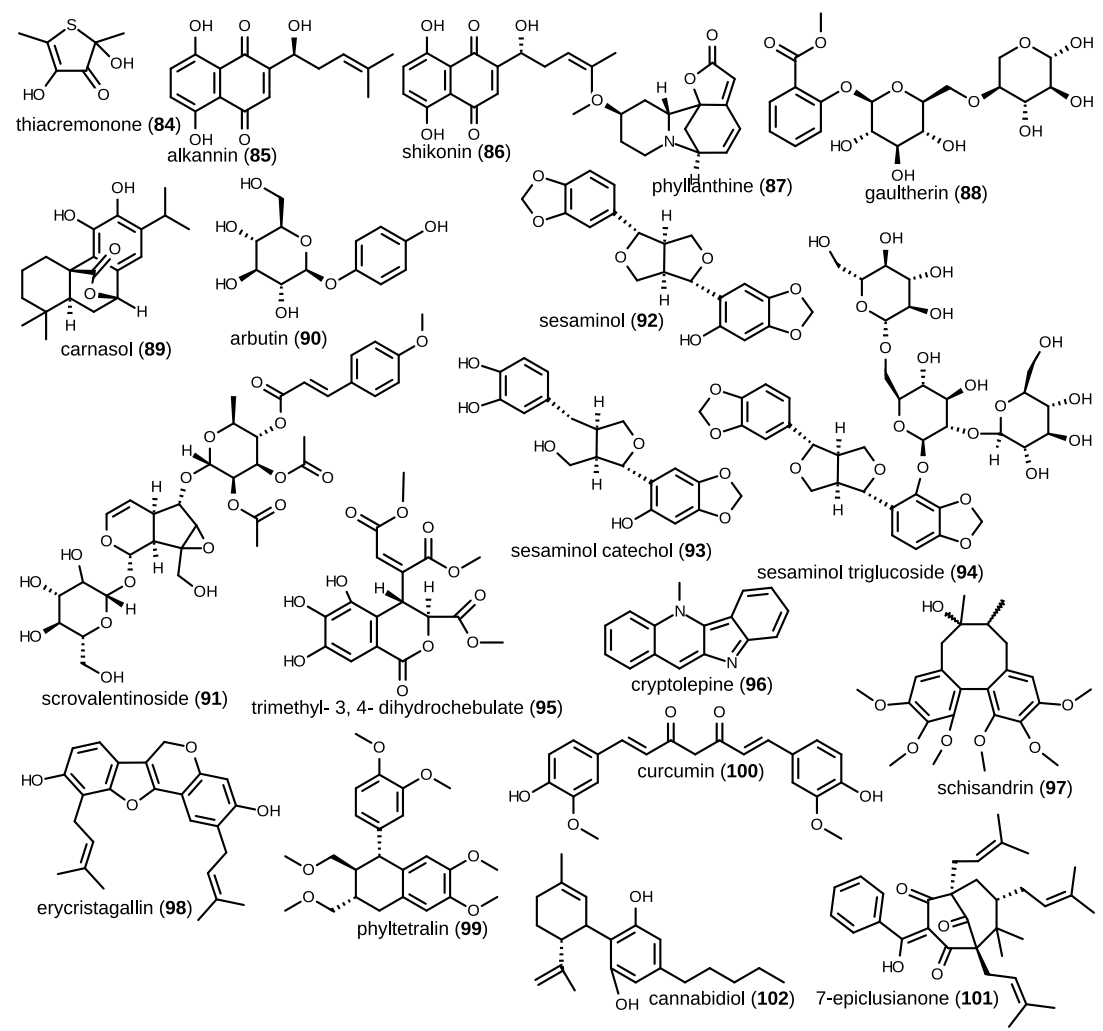

Figure 2.11: Miscellaneous - natural products. 
Table 2.12: Miscellaneous - natural products (n=19).

\begin{tabular}{|c|c|c|c|c|}
\hline Name & CAS RN & $M W$ & $\log D(p H 7.4)$ & tPSA \\
\hline Thiacremonone (84) & $96504-28-8$ & 160.19 & 0.06 & 57.53 \\
\hline Alkannin (85) & $517-88-4$ & 288.30 & 2.98 & 94.83 \\
\hline Shikonin (86) & $517-89-5$ & 288.30 & 2.98 & 94.83 \\
\hline Phyllanthine $(87)$ & 20072-02-O & 247.29 & -1.27 & 38.77 \\
\hline Gaultherin $^{a}$ (88) & $490-67-5$ & 446.41 & -1.74 & 184.60 \\
\hline Carnasol (89) & $5957-80-2$ & 330.42 & 4.57 & 66.76 \\
\hline Arbutin $^{a}(90)$ & $497-76-7$ & 272.25 & -0.90 & 119.61 \\
\hline Scrovalentinoside (91) & $214981-42-7$ & 752.72 & -0.40 & 247.96 \\
\hline Sesaminol (92) & $74061-79-3$ & 370.36 & 2.14 & 75.61 \\
\hline Sesaminol catechol (93) & $1171818-97-5$ & 360.36 & 1.93 & 108.61 \\
\hline Sesaminol triglucoside (94) & $157469-83-5$ & 856.78 & -3.67 & 313.06 \\
\hline Trimethyl-3,4-dihydrochebulate (95) & $154702-77-9$ & 396.30 & 0.75 & 165.89 \\
\hline Cryptolepine (96) & $480-26-2$ & 232.29 & 3.86 & 17.82 \\
\hline Schisandrin (97) & $7432-28-2$ & 432.51 & 3.39 & 75.61 \\
\hline Erycristagallin (98) & $92533-56-7$ & 390.46 & 6.09 & 62.83 \\
\hline Phyltetralin (99) & $123048-17-9$ & 416.51 & 3.36 & 55.38 \\
\hline Curcumin $^{\mathrm{b}}$ (100) & $458-37-7$ & 368.39 & 4.12 & 93.06 \\
\hline 7-epiclusianone (101) & $250275-46-8$ & 502.70 & 7.41 & 71.44 \\
\hline Cannabidiol $^{\mathrm{c}}$ (102) & $13956-29-1$ & 314.47 & 314.47 & 40.46 \\
\hline Median values & & 368.39 & 2.98 & 75.61 \\
\hline
\end{tabular}


Table 2.13: Miscellaneous - reference compounds $(n=25)$.

\begin{tabular}{|c|c|c|c|c|}
\hline Name & CAS RN & MW & $\log \mathrm{D}(\mathrm{pH} 7.4)$ & tPSA \\
\hline AMT (109) & $1121-91-1$ & 130.21 & -1.19 & 38.38 \\
\hline $\mathrm{BHT}^{\mathrm{c}}$ (110) & $128-37^{-0}$ & 220.36 & 5.27 & 20.23 \\
\hline Tiron free acid (111) & $149-46-2$ & 270.23 & -4.40 & 149.20 \\
\hline Captoprile $^{\text {(112) }}$ & $62571-86-2$ & 217.28 & -2.42 & 57.61 \\
\hline 1400W (113) & $180001-34-7$ & 177.25 & -4.28 & 61.90 \\
\hline Aspirine (114) $^{e}$ & $50-78-2$ & 180.16 & -2.16 & 63.60 \\
\hline Ascorbic acid $^{e}(\mathbf{1 1 5})$ & $50-81-7$ & 176.12 & -4.83 & 107.22 \\
\hline Metformin $^{e}(116)$ & $657-24-9$ & 129.17 & -5.62 & 88.99 \\
\hline Celecoxib $^{e}$ e (117) & $169590-42-5$ & 381.37 & 4.01 & 77.98 \\
\hline Trolox (118) & $53188-07-1$ & 250.29 & 0.38 & 66.76 \\
\hline Naphthazarin (119) & $475-38-7$ & 190.15 & 2.16 & 121.00 \\
\hline Zileuton $^{f}(\mathbf{1 2 0})$ & $111406-87-2$ & 236.29 & 1.99 & 66.56 \\
\hline Pyrilamine $^{\mathrm{e}}$ (121) & $91-84-9$ & 271.36 & 1.54 & 28.60 \\
\hline Omeprazole $^{e}(\mathbf{1 2 2})$ & $73590-58-6$ & 345.42 & 2.43 & 77.10 \\
\hline SB $203580(\mathbf{1 2 3})$ & $152121-47-6$ & 377.44 & 3.14 & 58.64 \\
\hline Tenidap sodium (124) & $119784-94-0$ & 343.74 & -0.06 & 83.63 \\
\hline Indomethacin $^{e}$ (125) & $53-86-1$ & 257.79 & 0.27 & 68.53 \\
\hline Cytochalasin B (126) & $14930-96-2$ & 479.62 & 4.08 & 95.86 \\
\hline TPCK (127) & $402-71-1$ & 351.85 & 3.89 & 63.24 \\
\hline Tacrolimus $^{\mathrm{e}}$ (128) & $104987-11-3$ & 804.03 & 5.59 & 178.36 \\
\hline Ketotifen fumarate $^{e}$ (129) & $34580-14-8$ & 425.50 & 3.16 & 20.31 \\
\hline Cyproheptadine $^{e}$ (130) & $129-03-3$ & 287.41 & 3.65 & 3.24 \\
\hline Paclitaxel $^{\text {e }}(\mathbf{1 3 1})$ & $33069-62-4$ & 853.92 & 3.54 & 221.29 \\
\hline L-NMMA (132) & $17035-90-4$ & 188.23 & -4.56 & 111.23 \\
\hline L-NAME (133) & $50903-99-6$ & 233.23 & -5.70 & 149.59 \\
\hline Median values & & 270.23 & 1.54 & 68.53 \\
\hline
\end{tabular}




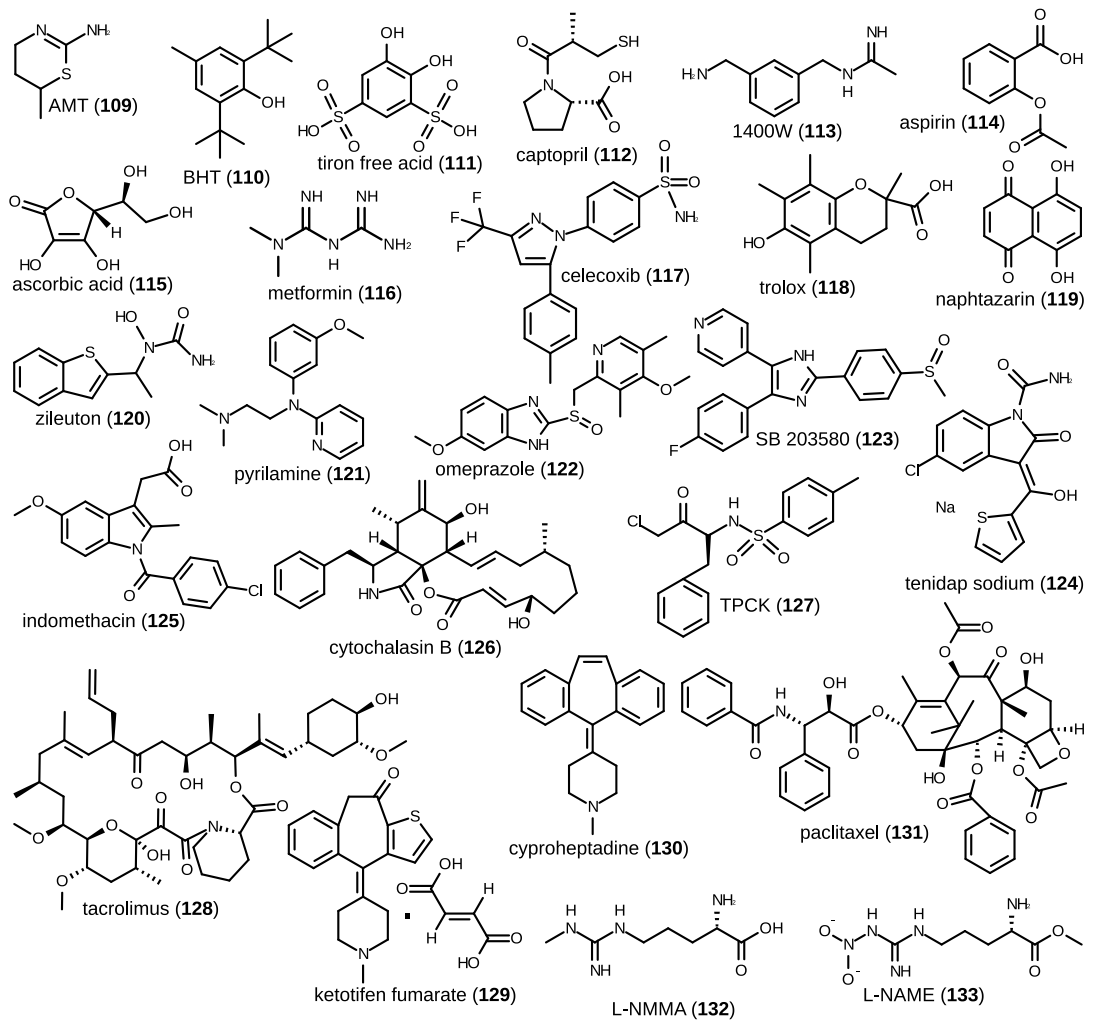

Figure 2.12: Miscellaneous - reference compounds. 


\subsection{ORAL BIOAVAILABILITY}

The 102 different natural products as well as the 31 reference compounds show considerable variation in physicochemical characteristics. These characteristics in turn, determine the distribution over the body after administration. The preferred administration route for systemic anti-inflammatory agents is via the enteral pathway since this offers highest patient convenience, lowest costs and minimal invasiveness. In general, small compounds with sufficient lipophilicity can pass membranes by a concentration gradient driven diffusion, whereas large, charged polar and/or protein-bound compounds cannot [77]. The physicochemical properties that are important for the prediction of oral bioavailability are critical to identify lead compounds that already possess drug-like properties $[77,10]$. To predict the oral bioavailability of the studied compounds, physicochemical properties were calculated by ChemAxon's Marvin software [36]. MW, $\log \mathrm{D}$ at $\mathrm{pH} 7.4$ and topological polar surface area (tPSA) can be found in Tables 2.3, 2.4, 2.5, $2.6,2.7,2.8,2.9,2.10,2.11,2.12,2.13$ and a complete properties list in supplementary Table $S_{1}$.

\subsubsection{Molecular weight}

Large molecules cannot diffuse through cell membranes [10], therefore, the first important parameter for oral bioavailability is the size of the molecule. One way determining the size of a molecule is using its $M W$. As defined by Lipinski, in his well-known 'rule of 5 ', the MW should preferably not exceed $500 \mathrm{Da}$ [46].

The MW of all natural products in this dataset ranges from 126.11 to 907.49 , with a median of 322.45 . $85 \%$ is less than 500 , staying within Lipinski's limit. For comparison, the $\mathrm{MW}$ of the reference compounds is similar with a range of 129.17 to 853.92 and a median of 278.35. However, the total percentage of reference compounds which is less than 500, with 94\% higher and only tacrolimus (128) and paclitaxel (131) do exceed this limit.

The median MW of the stilbenes group is the smallest and that of the porphyrins the largest. The median $M W$ of the porphyrins is with 889.36 exceeding the 500 limit and therefore, this structural group is not suitable for classical drug development. In summary the median MW for the natural products is: porphyrins > sterols > flavonoidsditerpenoids $>$ miscellaneous $>$ catechol derivativescoumarins $>$ stilbenes. 


\subsubsection{Lipophilicity}

In order to have acceptable oral bioavailability, compounds should be able to cross membranes. This requires an intermediate lipophilicity. High hydrophobicity has been suggested to be more frequently associated with adverse toxicological outcomes [76]. The lipophilicity of a molecule is determined by the partitioning between the octanol and water phase and is defined by the logarithm of this ratio as $\log \mathrm{P}$ (for the neutral molecular species) or as $\log \mathrm{D}$ (for the molecular species at a specific $\mathrm{pH}$ ). Meaning that if a molecule is non-ionizable, the $\log \mathrm{P}$ is equal to the $\log \mathrm{D}$ at every $\mathrm{pH}$. Since the $\log \mathrm{D}$ takes $\mathrm{pH}$ into account, it is better to use this for comparison $[76,38]$ and hence is used in this analysis.

As defined by Lipinski as part of his 'rule of 5 ', the (calculated) $\log P$ should be less than 5 for good oral bioavailability. There is no lower logP limit set, since this can supposedly be rescued by medicinal chemistry modifications [46].

The median $\log \mathrm{D}$ at $\mathrm{pH} 7.4$ of this natural product dataset is 2.41, ranging from -3.67 to 12.48 of which $84 \%$ is less than five and would pass Lipinski's rule. For comparison, the reference compounds have a median of 1.68 with a similar percentage of reference compounds (90\%) that pass Lipinski's rule for lipophilicity.

Examining the $\log \mathrm{D}$ at $\mathrm{pH} 7.4$ values in the various structural classes of the natural products, shows that the coumarins are in general the most hydrophilic and the porphyrins the most lipophilic structures. The order of natural products sorted by median lipophilicity is: porphyrins $>$ sterols $>$ catechol derivatives $>$ stilbenes $>$ miscellaneous $>$ flavonoids $>$ diterpenoids $>$ coumarins.

\subsubsection{Hydrogen bond donors and acceptors}

The hydrogen bond donors and acceptors are also part of Lipinski's 'rule of 5 ' and should be less than five for the donors and less than ten for the acceptors [46]. Hydrogen bonding is considered important for the bioavailability because for a molecule to be able to cross a membrane, the hydrogen bonds with the aqueous environment should first be broken [77].

The number of hydrogen bond donors for the natural products is ranging from 0 to 10 . The vast majority, $88 \%$, has equal to or less than five donors. For comparison, the reference compounds all have less than five donors. The number of hydrogen bond acceptors of the natural products ranges from 1 to 22 , of which again the majority, $90 \%$, comply with Lipinski's rule. Comparing to the reference compounds, all but tacrolimus (128), 97\%, have less than ten acceptors. 
The number of hydrogen bond donors and acceptors is for all natural products in the coumarins and the catechol derivatives structural groups less than five or less than ten, respectively. In the remaining groups, the compounds not complying to the rule all bear one or more sugar moieties.

\subsubsection{Polar surface area}

The polar surface area (PSA), which is the surface sum of all polar atoms (oxygen, nitrogen) in a molecule, provides, like the $M W$, information on the size of the molecule. In general, the smaller the PSA, the easier the molecule can travel through a membrane [62]. It has experimentally been found by Veber et al., in testing more than thousand rats, that the value for the PSA should be less than $140 \AA^{2}$ for crossing normal cell membranes and less than $70 \AA^{2}$ for crossing the blood brain barrier [74].

\subsubsection{Rotatable bonds}

Together with the importance of the PSA, the number of rotatable bonds is a good predictor of oral bioavailability and should not exceed ten, as defined by Veber et al.. In practice,it appears that only a few compounds with a MW above 500 have a low number of rotatable bonds [74].

The total range of rotatable bonds for the natural products is 0 to 23 , with $85 \%$ having less than ten rotatable bonds. For comparison, the reference compounds have a range of 0 to 14 , with $94 \%$ less than ten rotatable bonds. Only $\alpha$-tocopherol (104) and paclitaxel (131) have more rotatable bonds.

All natural products in the coumarins, diterpenoids, stilbenes and flavonoids groups do comply to Veber's rule and have less than ten rotatable bonds. For the sterols, only pepluanone (50) has more than ten rotatable bonds and for the rest group only scrovalentinoside (91) and sesaminol triglucoside (94), notably with a sugar moiety, have less than ten rotatable bonds. For both the catechol derivatives and the porphyrins, most natural products have more than ten rotatable bonds.

\subsubsection{Aromatic rings}

Aromatic rings have a strong impact on water solubility, but there is increasing evidence that the number of aromatic rings also influences drug applicability within the appropriate $\log \mathrm{D}$ range. As experimentally determined, the optimal aromatic ring number should be equal to or less than three [68]. 
The total range of aromatic rings for the natural products is 0 to 4, with only $6 \%$ with more than three rings. The reference compounds show the same range and perform with $3 \%$ with more than three rings slightly better than the natural products.

The few exceptional natural products that do exceed the maximum optimal number of aromatic bonds are, again, all four porphyrins, tiliroside (78) from the flavonoids and cryptolepine (96) from the miscellaneous group. All but cryptolepine (96) have also a high MW and do not pass Lipinski's rule.

\subsection{ANTI-INFLAMMATORY ACTIVITY}

Anti-inflammatory activity of the natural products tested in the papers collected for this systematic review was generally assessed using similar assays under comparable, albeit slightly different, conditions. In total, 25 different assays were used in which the inhibitory activity of at least two natural products was determined. Assays could be categorized according to the different aspects of the inflammatory response that were examined, including the in vitro inhibition of oxidation, inhibition of the production of cytokines, eicosanoids, or various other inflammatory mediators and in in vivo edema experiments. Performance of all natural products and reference compounds for all assays can be found in supplementary Tables S2 -S8. Anti-inflammatory performance of the natural products was determined using the median rather than the mean values of the maximum inhibition and corresponding concentrations, to correct for outliers in the dataset. Every compound with values higher than the median of the maximum inhibition and values lower than the median of the corresponding concentration, performed 'better than median'.

\subsubsection{Anti-oxidant capacity}

In vitro anti-oxidant activity was mainly assessed by the ability of the compounds to inhibit or scavenge free radicals $\left(\mathrm{NO}, \mathrm{DPPH}, \mathrm{O}_{2}{ }^{-} \cdot \mathrm{OH}\right.$ and ROS), inhibit lipid oxidation (LPO) and iNOS enzyme upregulation (Tables 2.14 and $\mathrm{S}_{3}$ ).

Nitric oxide (NO) regulates various physiological and pathophysiological pathways, including host defense and immune responses. As a reaction to inflammatory stimuli, high levels of NO are produced, mediating pro-inflammatory and destructive effects [44]. In vitro NO inhibition was the most often used assay in the studies, with 52 natural products from 17 different studies $[1,4,7,12,16,17,21,23,24$, $29,32,35,41,43,48,65,75]$. Of the natural products used in this as- 
say, $27 \%$ were better than median, none of the stilbenes performed better and the porphyrins were not tested with this assay. Methyl breviolincarboxylate (16) of the coumarins was the strongest NO scavenger, whereas bergapten (2), heraclenol (10), heraclenin (11) of the coumarins, $\alpha$-chaconine (51) of the sterols, andrograpanin (54), 14-deoxy11,12-didehydroandrographolide (58), and neoandrographolide (59) of the diterpenoids did not show any scavenging effects at the tested concentrations. Twelve reference compounds were included, originating from ten different studies $[1,7,12,17,24,29,32,35,41,43]$ of which $33 \%$ performed better than the natural products median values.

The $\alpha, \alpha$-diphenyl- $\beta$-pycryl-hydrazyl (DPPH) free radical is an important and often used cell free assay that is used to determine the scavenging activity of molecules [40]. DPPH radical inhibition was the second most used assay with twenty-three natural products examined across seven studies $[13,16,21,23,56,70,71] 78 \%$ was superior over the median values. Erycristagallin (98) of the rest group was the best performer and sugiol (39) of the sterols and pinocembrin (64) and gnaphaliin (67) of the flavonoids did not show any scavenging effect at the tested concentrations. Five reference compounds from four studies $[16,21,70,71]$ were used, including the classic DPPH scavenging reference BHT (110). $80 \%$ of the reference compounds performed better than the natural products median values.

The superoxide anion $\left(\mathrm{O}_{2}^{-} \cdot\right)$ is a critical factor in several physiological processes, such as signal transduction (through cGMP), smooth muscle relaxation and control of ventilation. However, high concentrations have detrimental effects and are associated with chronic inflammatory diseases [20]. This $\mathrm{O}_{2}{ }^{-}$. inhibition assay was used to test the scavenging properties of fifteen natural products, from four different studies $[61,70,72,21]$. Of these natural products, $40 \%$ performed better than the median values. The natural reference compound pyrogallol (33), outperformed the median values, whereas the other natural reference compound fraxetin (20) did not. However, the reported $\mathrm{IC}_{50}$ was in the same range as the $\mathrm{IC}_{50}$ s of natural products 22, 23, 24 and 25 with high maximum inhibitory percentages. The only synthetic reference compound, tiron free acid (111), had a reported $\mathrm{IC}_{50}$ of such a high concentration, that it did not seem to be a proper reference choice.

\subsubsection{Cytokine/chemokine production inhibition}

Inhibition of in vitro cytokine/chemokine (IL-6, TNF- $\alpha$, IL-1 $\beta, N F-\kappa \beta$, IL-2 and IL-8) production was measured (Tables 2.15 and $\mathrm{S}_{4}$ ). In contrast to the anti-oxidant assays, the natural products from the catechol derivatives were hardly tested, and if tested, these compounds were not performing better than median. In general, both the natural prod- 
ucts and reference compounds were predominantly from the sterols and rest groups.

IL-6 is an important mediator of the JAK/STAT pathway, and a regulator of T-cell regulation and activation, promoting a pro-inflammatory environment[54]. IL-6 production inhibition is most often used to establish the cytokine inhibiting performance of the natural products in this systematic review. Twenty natural products from five different studies $[8,12,23,29,37]$ were used, of which $35 \%$ performed better than their median values. As reported before, 54, 58 and 59 of the diterpenes did not show any inhibitory effect, as was also the case for 7-o-methylwogonin (66) of the flavonoids. The reference compounds were two well-known synthetic corticosteroids, hydrocortisone (106) and dexamethasone (108). 108 was used in two different studies, but only once it performed better than the median inhibitory values of the natural products.

TNF- $\alpha$ is a stimulator of NF- $\kappa \beta$, an important cytokine in inflammation and associated with numerous chronic inflammatory diseases [33]. In this systematic review, the ability to inhibit the production of TNF- $\alpha$ in vitro was determined for nineteen different natural products, from seven studies $[13,16,21,23,56,70,71]$ of which $32 \%$ performed better than median. Again, 16 was the strongest inhibitor. Four different reference compounds were used from five studies $[8,13,17,29$, 43]. 108 was used in two studies and performed both times better than the median values of the natural products. Tested concentrations of the well-performing reference compounds were around $1 \mu \mathrm{M}$, about thirty times lower than the concentrations of the well-performing natural products. Only bergapten (2) in its dimeric form was in the same concentration range, however its maximum inhibition percentage was relatively poor.

Table 2.14: Anti-inflammatory performance of natural products in oxidation assays. Natural products tested per assay are given and underlined compounds always performed better than median.

\begin{tabular}{|c|c|c|}
\hline Assay (n) & Median values & Better than median \\
\hline $\mathrm{NO}(52)$ & $57.8 \% \& 63.0 \mu \mathrm{M}$ & $26.9 \%: \underline{13}, \underline{16}, 22,23, \underline{24}, 47,55,56,69,77, \underline{80}, \underline{87}, \underline{95}, \underline{99}$ \\
\hline DPPH (23) & $73.5 \% \& 100.0 \mu \mathrm{M}$ & $26.9 \%: \underline{16}, 22, \underline{23}, \underline{24}, \underline{25}, 31,53,61,62,63,69, \underline{70}, 78, \underline{80}, \underline{87}, \underline{95}, 98, \underline{99}$ \\
\hline $\mathrm{O}_{2}-(15)$ & $32.1 \% \& 100.0 \mu \mathrm{M}$ & $40.0 \%: 22, \underline{23}, 24,25, \underline{33}, 78$ \\
\hline $\mathrm{OH}(9)$ & $90.0 \% \& 551.0 \mu \mathrm{M}$ & $33.3 \%: \underline{23}, \underline{24}, \underline{25}$ \\
\hline LPO (9) & $50.0 \% \& 100.0 \mu \mathrm{M}$ & $77.8 \%: 21,61,62,63,78,85,86$ \\
\hline iNOS (7) & $82.0 \% \& 100.0 \mu \mathrm{M}$ & $43.9 \%: 50,74,84$ \\
\hline $\operatorname{ROS}(4)$ & $30.3 \% \& 167.4 \mu M$ & $0.0 \%:-$ \\
\hline
\end{tabular}




\subsubsection{Eicosanoids inhibition}

Inflammatory mediators from the eicosanoids group include prostaglandins and enzymes $\left(\mathrm{PGE}_{2}, \mathrm{PGD}_{2}, \mathrm{LTB}_{4}, \mathrm{TXB}_{2}, \mathrm{COX}-1, \mathrm{COX}-2\right.$ and LOX) (Tables 2.16 and $\mathrm{S}_{5}$ ). The diterpenes group was largely represented in all assays and performed in $43 \%$ of the cases better than median.

$\mathrm{PGE}_{2}$ is a lipid mediator from the arachidonic acid pathway, promoting (among more) acute inflammation through vasodilation and increased blood flow, leading to the typical inflammatory red flare and heat [39]. $\mathrm{PGE}_{2}$ inhibition was determined for seventeen natural products from eight different studies [12, 16, 17, 21, 24, 32, 43, 65] of which $29 \%$ performed better than their median values. Notably, once again, for 54, 58 and 59 no inhibition at all was reported, which was also the case for tirucallol (44). The reference compounds from the miscellaneous group, AMT (109) and celecoxib (117), performed better than the median of the natural products whereas 108 from the sterols group, did not.

$\mathrm{LTB}_{4}$ is a small lipid molecule derived from arachidonic acid as well. It is one of the most potent chemoattractants and activates leukocytes, making it a key activator in inflammatory diseases [78]. Inhibition of $\mathrm{LTB}_{4}$ was measured for fifteen natural products from six different studies $[6,7,12,34,56,70]$ of which $27 \%$ performed better than median. Once more, all but one of the diterpenes including 54, 58 and 59 did not show any inhibitory activity, only the $\mathrm{IC}_{50}$ reported for dehydrocostic acid (60) was better than median. Pinocembrin (64) from the flavonoids was the strongest inhibitor with a high maximum inhibitory percentage at a low concentration. All used references were from the miscellaneous group and performed better than the median of the natural products.

Table 2.15: Anti-inflammatory performance of natural products in cytokine assays. Natural products tested per assay are given and underlined compounds always performed better than median.

\begin{tabular}{|c|c|c|}
\hline Assay (n) & Median values & Better than median \\
\hline IL-6 (20) & $62.0 \% \& 57.0 \mu \mathrm{M}$ & $35.0 \%: \underline{16}, 55,56, \underline{80}, 87, \underline{95}, 99$ \\
\hline TNF- $\alpha(19)$ & $69.5 \% \& 50.0 \mu \mathrm{M}$ & $31.6 \%: \underline{\mathbf{1 6}}, \underline{\mathbf{7 1}}, \underline{\mathbf{8 0}}, \underline{87}, \underline{95}, \underline{99}$ \\
\hline IL-1 $\beta(9)$ & $41.3 \% \& 57.0 \mu \mathrm{M}$ & $33.3 \%: 39,55,56$ \\
\hline$N F-\kappa \beta(8)$ & $55.2 \% \& 62.4 \mu \mathrm{M}$ & $12.5 \%: 80$ \\
\hline IL-2 (4) & $28.0 \% \& 13.2 \mu \mathrm{M}$ & $0.0 \%:-$ \\
\hline Il-8 (3) & $21.0 \% \& 1.2 \mu \mathrm{M}$ & $33.3 \%: 5 \mathbf{1}$ \\
\hline
\end{tabular}




\subsubsection{Other mediators}

The commonly used histamine release inhibition assay and the less known inhibition of elastase assay did not fit in any of the previous inflammatory categories (Tables 2.17 and S6).

Histamine is extensively studied in medicine and important in acute allergic inflammatory reactions. It is excreted by several cell types (i.e. mast cells, lymphocytes) and can attract important effector cells, leading to chronic inflammation [2]. Histamine release inhibition in vitro was determined for seventeen natural products from four different studies $[5,6,12,25]$. About a quarter performed better than median. All natural products from the diterpenes, including again 54, 58 and 59 did not show any histamine release inhibition. The natural reference compound luteolin (72) from the flavonoids and the synthetic reference compounds pyrilamine (121) and ketotifen fumarate (129) from the miscellaneous group performed better than the median values of the natural products.

Table 2.16: Anti-inflammatory performance of natural products in eicosanoids assays. Natural products tested per assay are given and underlined compounds always performed better than median.

\begin{tabular}{|c|c|c|}
\hline Assay (n) & Median values & Better than median \\
\hline $\mathrm{PGE}_{2}$ (17) & $66.0 \% \& 57.0 \mu \mathrm{M}$ & $23.5 \%: 23, \underline{24}, \underline{25}, 55$ \\
\hline $\mathrm{LTB}_{4}(15)$ & $25.1 \% \& 57.0 \mu \mathrm{M}$ & $26.7 \%: 60,64,67, \underline{71}$ \\
\hline $\mathrm{TXB}_{2}(8)$ & $34.3 \% \& 57.0 \mu \mathrm{M}$ & $33.3 \%: 55,56$ \\
\hline $\operatorname{COX}-2(7)$ & $68.7 \% \& 100.0 \mu \mathrm{M}$ & $42.9 \%: 74,84,97$ \\
\hline $\operatorname{COX}-1(3)$ & $8.1 \% \& 100.0 \mu \mathrm{M}$ & $66.7 \%: 47,60$ \\
\hline LOX (2) & $50.0 \% \& 39.6 \mu \mathrm{M}$ & $50.0 \%: 83$ \\
\hline $\mathrm{PDG}_{2}(2)$ & $78.6 \% \& 55.0 \mu \mathrm{M}$ & $33.3 \%: \underline{71}$ \\
\hline
\end{tabular}

Table 2.17: Anti-inflammatory performance of natural products in other assays. Natural products tested per assay are given and underlined compounds always performed better than median.

\begin{tabular}{lll}
\hline Assay (n) & Median values & Better than median \\
\hline His (17) & $8.5 \% \& 100.0 \mu \mathrm{M}$ & $17.6 \%: \mathbf{6 1}, \mathbf{6 6}, \underline{\mathbf{7 1}}$ \\
Elas (2) & $85.0 \% \& 55.0 \mu \mathrm{M}$ & $0.0 \%:-$ \\
\hline
\end{tabular}




\subsubsection{In vivo edema inhibition}

In vivo edema experiments, mimicking acute inflammation in either paws (both mouse and rat) or ears (mouse only) had very similar experimental conditions in this systematic review and could be compared to each other (Tables 2.18 and $S_{7}$ ). In general, tested natural products were from the sterols, flavonoids and miscellaneous structural groups, and the reference compounds were exclusively from the sterols and miscellaneous groups. Reference compound choice is comparable with that of the IL-6 production inhibition assay.

Swelling of the mouse ear is a model that since the 1980s is regularly used to show the inhibitory capacity of a drug in acute inflammation [28]. For fourteen natural products, from nine different studies [4, 7, 15, $24,34,48,56,58,70]$, the ability to reduce ear edema was determined. Only $21 \%$ of the natural products performed better than median and were mainly from the sterols. The reference compounds were identified in eight different studies [7, 15, 24, 34, 48, 56, 58, 70], in which indomethacin (125) was used in six studies, prednisolone (107) in two and dexamethasone (108) in one. Only 108 and one out of six times used $\mathbf{1 2 5}$ did perform better than the median values of the natural products.

Rat and mouse paw swelling, mostly carrageenan induced, is a widely used method to study acute inflammation [50]. Twelve natural products were tested for rat paw edema reduction, of which $25 \%$ performed better than median. The inhibitory activity of the natural products was measured in nine different studies $[1,4,16,17,22,42,58,59,65]$, and were mainly from the sterols. However, the strongest inhibitor was linolenic acid (29) from the catechol derivatives. No less than ten reference compounds were used, all originating from either the sterols or the rest groups. $50 \%$ of the reference compounds performed better than the median values of the natural products, however, only AMT (109) and TPCK (127) were used in multiple studies.

Reduction of mouse paw edema was examined for nine natural products, originating from six studies [32, 34, 45, 56, 70]. Interestingly, whereas rat paw swelling reduction was mainly tested with natural products from the sterols, none of the sterols group were included here. Natural products were predominantly from the flavonoids and more than half performed better than median. All but one reference compound performed better than the median values of the natural products and are from the sterols or miscellaneous groups. 


\subsection{BEST PERFORMING AND MOST PROMISING NATURAL PRODUCTS}

In this systematic review, a total of 102 natural products was studied. 42 natural products $(41 \%)$ showed higher potency than the median in at least one of the assays (Tables 2.14, 2.15, 2.16, 2.17 and 2.18, Figure 2.13). Of these, fourteen (14\% of total population and $33 \%$ of selection) performed better than median in all assays they were studied in.

These latter are: byakangelicol (13) and methyl breviolincarboxylate (16) of the coumarins, 8-gingerol (23), 10-gingerol (24) and 6-shogaol (25) and pyrogallol (33) of the catechol derivatives, quercetin (70), luteolin $(7 \mathbf{1})$, rutin $(80)$ and baicalein $(83)$ of the flavonoids and alkannin (85), phyllanthine (87), trimethyl-3,4-dihydrochebulate (95) and phyltetralin (99) of the rest group (Figure 2.13). In order of anti-inflammatory performance: $24>23$ (5 assays) $>16>25>71>95>87>80>99$ (4 assays) $>85$ ( 2 assays) $>70>33>13>83$ ( 1 assay).

Of these natural products that perform well in the anti-inflammatory tests, several have a low predicted oral bioavailability, in decreasing order $24>23>95>80$ (Figure 2.14). These molecules require parenteral administration, a medicinal chemistry approach to improve drug-like characteristics or encapsulation into a nanoparticle to enhance availability. The natural products which do comply to all bioavailability rules, as described in section 2.4, and even should be able to pass the blood brain barrier [74] are in decreasing order: $87>25>99>33$ (Figure 2.15). And since the natural reference compound pyrogallol (33) was used in only one assay, the most promising anti-inflammatory natural products with the most favorable bioavailability properties (i.e. 'best performers') are 6-shogaol (25) from the catechol derivatives and phyllanthine $(87)$ and phyltetralin (99) from the miscellaneous group. Narrowing this even further down, 25 and 99 are not in the defined 'Golden Triangle' area (Figure 2.16). This leaves phyllantine (87), which is on the border of the triangle, at the top of this natural product dataset.

Table 2.18: Anti-inflammatory performance of natural products in edema assays. Natural products tested per assay are given and underlined compounds always performed better than median.

\begin{tabular}{lll}
\hline Assay (n) & Median values & Better than median \\
\hline m_ear (14) & $65.8 \% \& 0.375 \mathrm{mg} / \mathrm{ear}$ & $21.4 \%: 42,43,98$ \\
r_paw (12) & $44.5 \% \& 15.2 \mathrm{mg} / \mathrm{kg}$ & $25.0 \%: 29,50,62$ \\
m_paw (9) & $62.6 \% \& 80.0 \mathrm{mg} / \mathrm{kg}$ & $55.6 \%: 60,64,74,78,85$ \\
\hline
\end{tabular}


The anti-inflammatory activity of the natural products was compared to well-known drugs. In general, the reference compounds were fairly well chosen, in only two cases (L-NAME (133) for ROS inhibition and trolox (118) for NO scavenging) there was no inhibition at all reported [35]. Half of the reference compounds performed in all tests better than the median values of the natural products. In decreasing order of anti-inflammatory performance: 125 ( 7 assays) $>108$ ( 7 out of 8 assays) $>109>110>128>119$ ( 2 assays) $>117>120>130$ ( 1 assay) $>121>104>129>115>112>105\left(\mathrm{IC}_{50}, 1\right.$ assay). Of these, only $\alpha-$ tocopherol (104), BHT (110) and tacrolimus (128) do have unfavorable bioavailability predictions.

Principally, the 'best performing' natural products are comparable to the reference compounds in terms of anti-inflammatory activity. However, in terms of bioavailability, the reference compounds are better, which might be obvious since these drugs are already designed to have favorable characteristics and are already marketed.

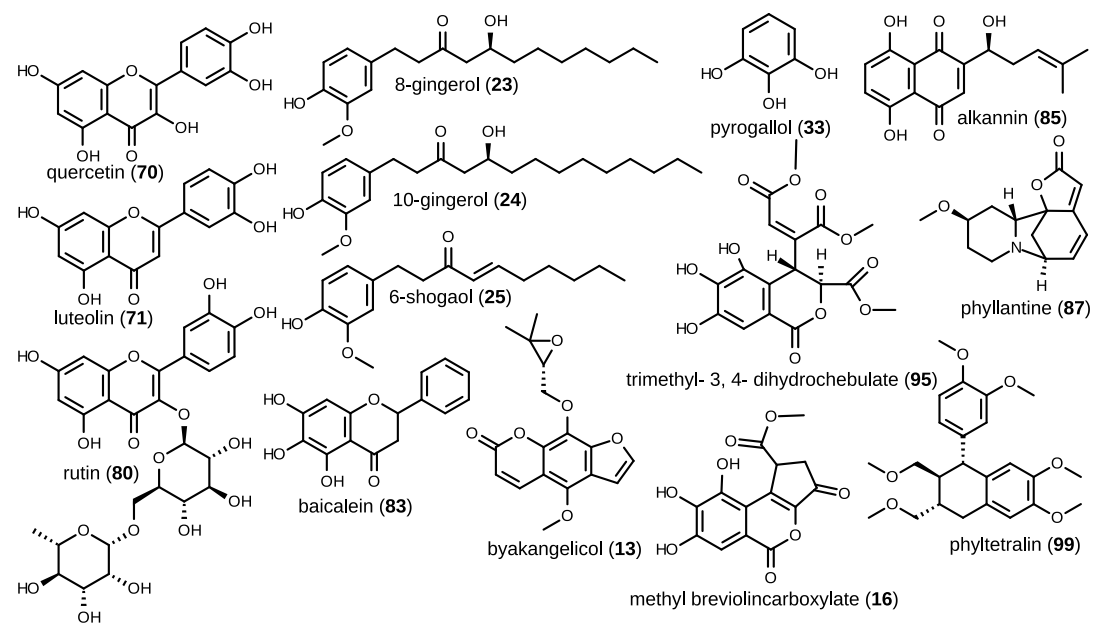

Figure 2.13: Structures of best anti-inflammatory natural products. These fourteen natural products performed better than median in the all the assays they were tested in. Structures are from the coumarins, catechol derivatives, flavonoids and miscellaneous structural groups. 


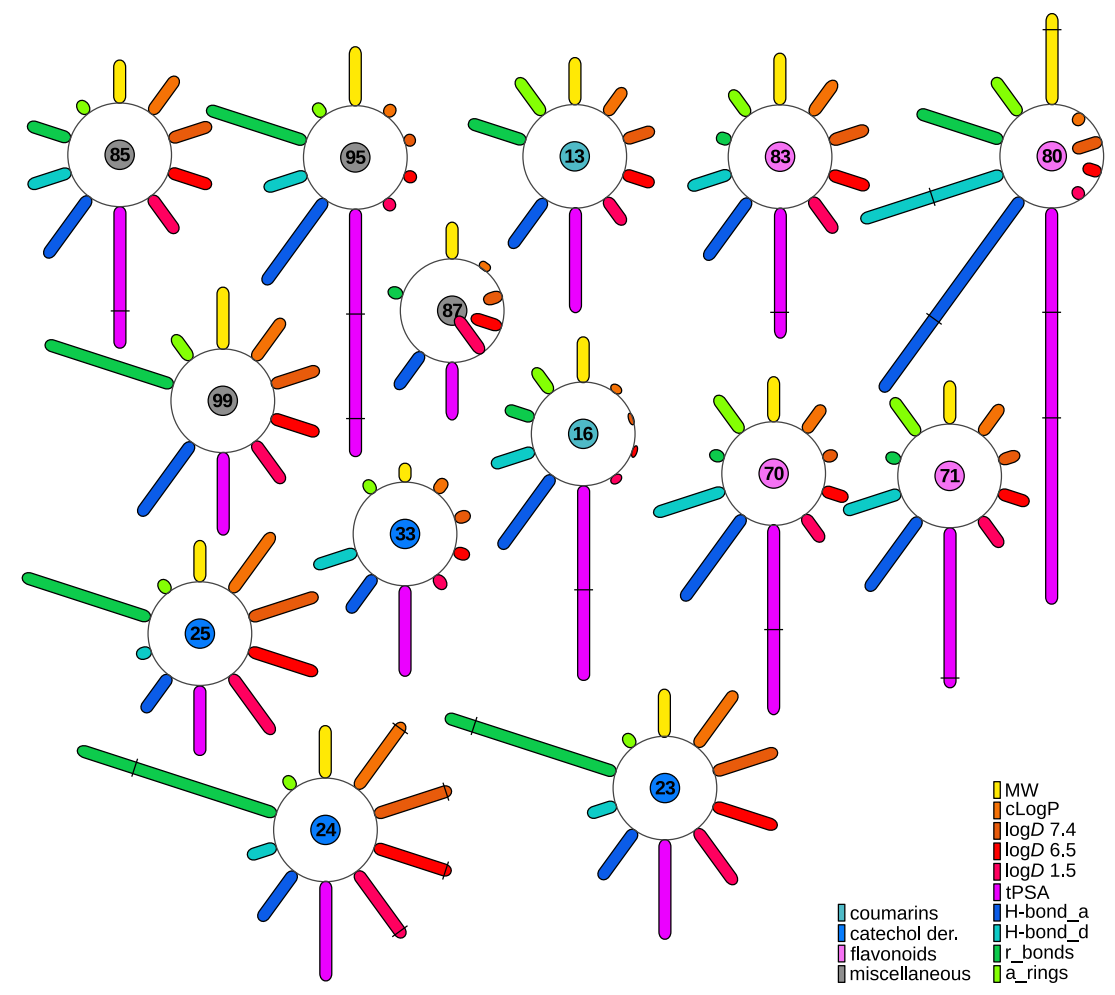

Figure 2.14: Physicochemical properties of best anti-inflammatory natural products. Displaying the physicochemical properties in flower plots, 83,84 reveals differences and similarities at first glance. The heart of the flower contains the compound number and is colored according to its structural group. The black line in the petals marks the limit for 'good' oral bioavailability of that specific property. $M W=$ molecular weight, $c \log P=$ calculated $\log P, t P S A=$ topological polar surface area, H-bond_a = hydrogen bond acceptor, H-bond_d = hydrogen bond donor, $r$ _bond = rotatable bonds and a_rings = aromatic rings 


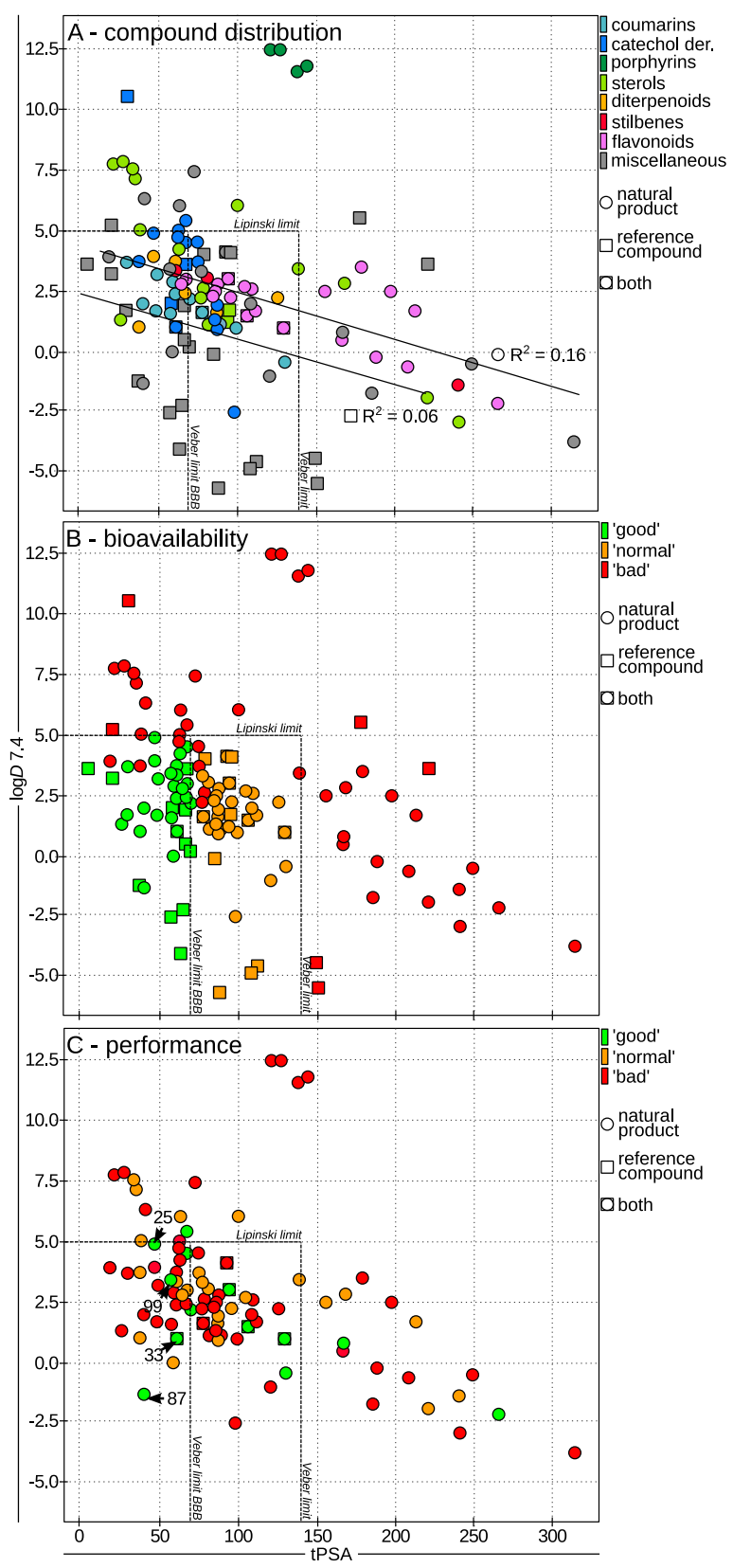

Figure 2.15: LogD plotted against tPSA. The maximum desirable Lipinski [46] and Veber [74] limits are indicated by the dashed lines. green = 'good' bioavailability, also passing the blood brain barrier, orange $=$ 'normal' bioavailability, passing membranes but not the blood brain barrier and red = 'bad' bioavailability, one or more properties are exceeding the provided limits. 


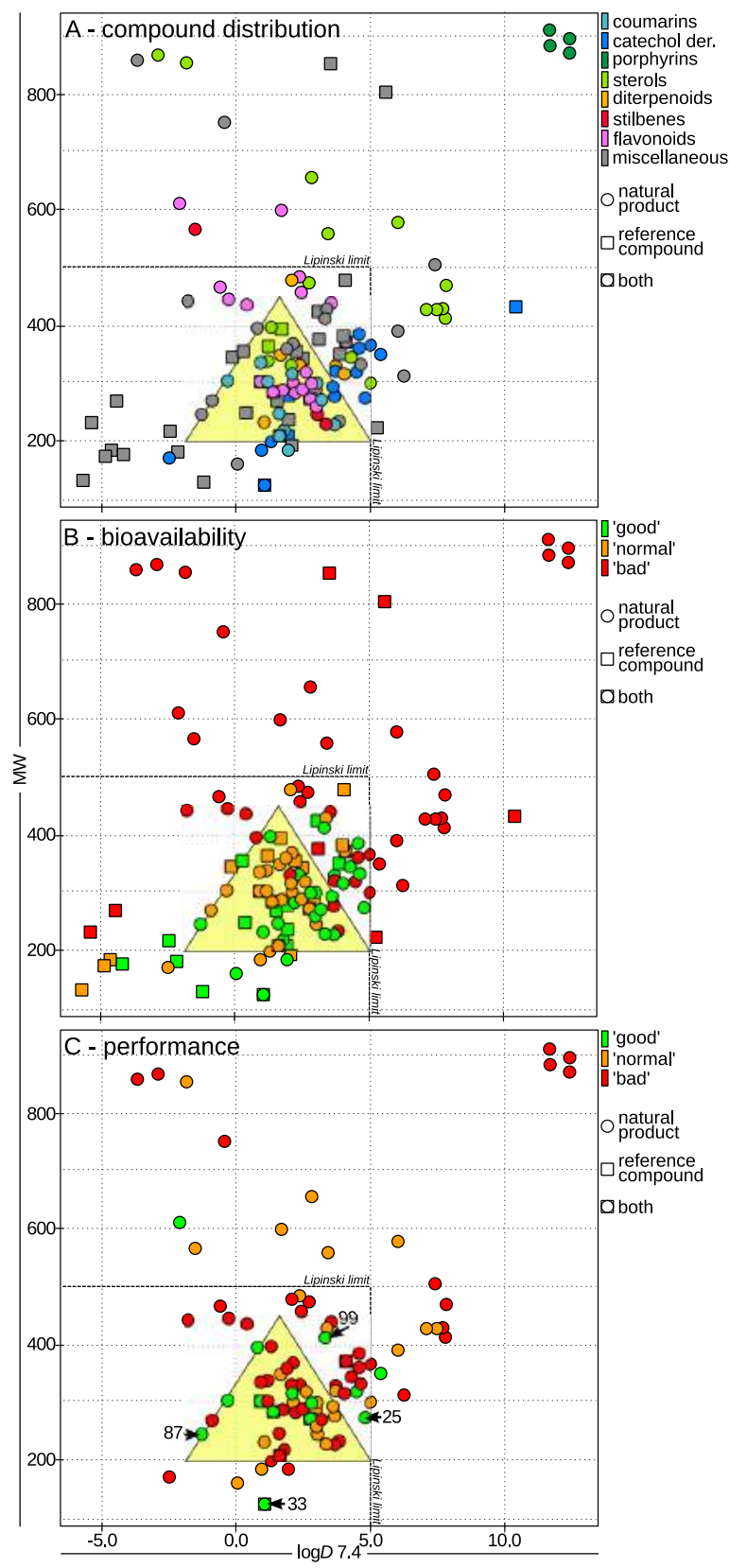

Figure 2.16: MW plotted against $\log \mathrm{D}$. The 'Golden Triangle' is a visualization tool to predict metabolically stable, permeable and potent drug candidates [38] and is placed from $\log D 1-5$ with the top at a $M W$ of 450 . The maximum desirable Lipinki limits [46] are indicated with dashed lines. green = 'good' bioavailability, also passing the blood brain barrier, orange $=$ 'normal' bioavailability, passing membranes but not the blood brain barrier and red = 'bad' bioavailability, one or more properties are exceeding the provided limits. 


\subsection{PREDICTING ANTI-INFLAMMATORY ACTIVITY OF NATURAL PRODUCTS}

Nowadays, drug discovery and development is driven by the use of computer simulation and biological activity prediction models [52]. Training datasets are used to help predict the activity of unknown compounds outside the training set. In order to build a robust structure activity relationship it is important to have a homogeneity in the dataset: experiments must have been performed with the same protocols with the same cell lines. Furthermore, to be able to find a structure activity relation, it is important that the dataset comprises the same chemical classes, and if not, that they have the same mechanism of action [18].

The relatively small dataset of this systematic review does not comply to these requirements because the anti-inflammatory activities were not determined following the same experimental design. Furthermore, the dataset has a large structural variety, which reflects the normal state of natural products [19]. In addition, the mechanism of action of most of the natural products in this systematic review remains elusive, hence grouping them by mechanism is impossible. These considerations make it impossible to build a single structure activity relationship to predict the anti-inflammatory activity for natural products in general. However, several structural and physicochemical correlations can be found in this dataset and give insight in the anti-inflammatory potential of natural products.

Existing parameters for predicting oral bioavailability are used in this dataset to filter out those compounds which, purely based on these properties, would not make good (anti-inflammatory) drugs. Since adequate oral bioavailability is one of the main obstacles for natural products, this is an important aspect for success.

The 'Golden Triangle', a drug visualization tool to predict metabolically stable, permeable and potent drug candidates [38] was used to aid in finding correlations (Figure 2.16). All best performing natural products (depicted in green) are on the 'Golden Triangle', or very close to it. Rutin (8o) is an exception, and lies even outside the Lipinski barrier. However, it is safe to say that in general once a natural product falls outside these barriers, the chances are substantial that it is not a strong anti-inflammatory compound. As expected from the bioavailability parameters and confirmed by the performed assays, the large porphyrins are not able to inhibit inflammation.

Another way of displaying the dataset and find outstanding natural products, is by plotting the $\log \mathrm{D}$ at $\mathrm{pH} 7.4$ against the tPSA as proposed by Waring et al.. [76] This dataset showed a low correlation for a linear relationship with $R^{2}$ of 0.16 , suggesting that the $\log D$ at 7.4 might be compromised at higher tPSA values. This correlation is even 
less obvious for the reference compound dataset, however, the trend is similar (Figure 2.15). Looking at the specific structural groups, the correlation becomes stronger in most cases; the sterols with $R^{2}$ of 0.62 , the coumarins with $R^{2}$ of 0.59 and the flavonoids, miscellaneous structures and catechol derivatives with an $R^{2}$ of respectively $0.46,0.44$ and 0.42 . The diterpenoids did not show any correlation and both the stilbenes and the porphyrins had correlations close to 1 , however, these groups are extremely small.

Of the fourteen natural products that perform better than median in all anti-inflammatory assays they participated in (Figure 2.13 and $2.14)$, ten (71\% of selection) did comply to the set bioavailability rules and four (29\% of selection, $4 \%$ of total dataset, Figure 2.17$)$ would even be suitable to pass the blood brain barrier. This is probably not a coincidence. Predicted suitable bioavailability enables these natural products to arrive at the desired place for their actions. Not all compounds scoring less than median in the anti-inflammatory assays have a disadvantageous bioavailability profile however, vice versa, two thirds of the well performing natural products do have an acceptable predicted bioavailability (Figures 2.15 and 2.16). Twenty-two natural products $(22 \%)$ never performed better in any anti-inflammatory assay and have very low predicted bioavailability (i.e. 'worst performers'). These include compounds from the catechol derivatives, porphyrins (all), sterols, flavonoids and miscellaneous structural groups, but not from the coumarins, diterpenoids and stilbenes. By far the largest group (70\%) in this dataset are mediocre natural products, either with weak anti-inflammatory performance or weak bioavailability.

The non-uniformity of the dataset and hence of the 'best' and 'worst' performing natural products (Figure 2.17), makes it hard to come to an unambiguous structure activity relationship. However we can conclude that, in order for a natural product to be a successful anti-inflammatory drug, it should not exceed the above mentioned oral bioavailability criteria, should not have sugar moieties and should ideally have one or more reactive enol moieties.

\subsection{DISCUSSION}

In this systematic review the anti-inflammatory potency of plant derived natural products has been examined. The activity of 102 natural products was compared in 25 anti-inflammatory assays. The top natural products, including both anti-inflammatory performance and bioavailability, are phyllanthine (87) followed by 6-shogaol (25), phyltetralin (99) and pyrogallol (33) (Figure 2.17). However, the majority of tested natural products does not show anti-inflammatory efficacy in 


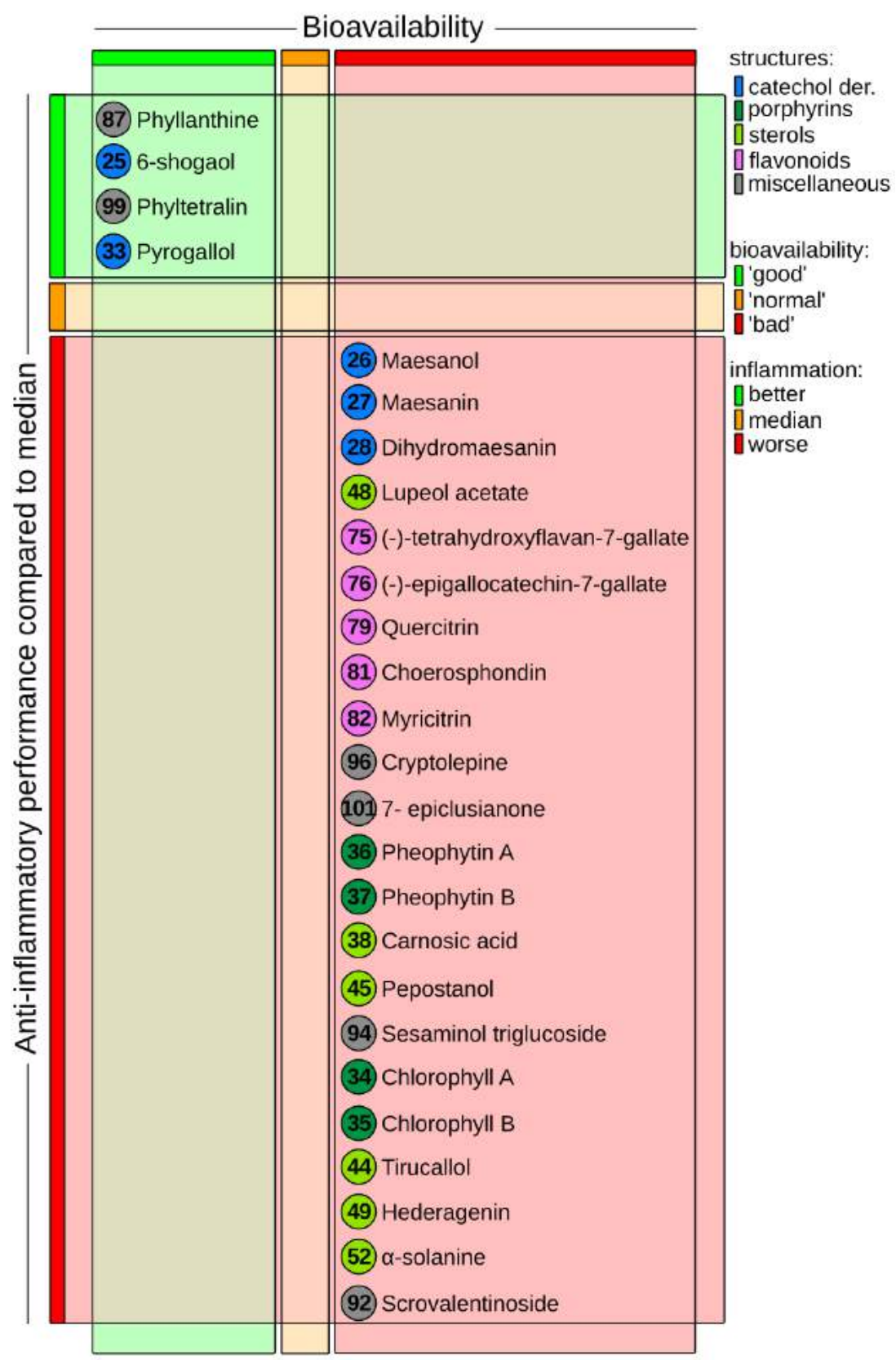

Figure 2.17: Performance spectrum. In the top left corner are the 'best' anti-inflammatory natural products with superb bioavailability, whereas in the bottom right corner are the natural products which do not show any anti-inflammatory efficacy and have unsatisfactory bioavailability. 
the tested assays and should probably not be further investigated. Natural products underperforming in the anti-inflammatory assays and failing one or more bioavailability limits are in increasing order of infirmity: maesanol (26), maesanin (27), dihydromaesanin (28), lupeol acetate (48), (-)-tetrahydroxyflavan-7-gallate (75), (-)-epigallocatechin-7gallate (76), quercitrin (79), choerosphondin (81), myricitrin (82), cryptolepine (96), 7-epiclusianone (101), pheophytin A (36), pheophytin B (37), carnosic acid (38), pepostanol (45), sesaminol triglucoside (94), chlorophyll A (34), chlorophyll B (35), tirucallol (44), hederagenin (49), $\alpha$-solanine (52) and scrovalentinoside (91) (Figure 2.17). A full list of the natural products from this analysis, ordered by anti-inflammatory potency, can be found in supplementary Table Sy.

\subsubsection{Natural products as anti-oxidants}

In the past decades, research has focused mainly on the anti-oxidant capacity of natural products, and of polyphenolic structures in particular. For example, the scavenging activity of 51 tannins was determined by Yokozawa et al. using the cell free DPPH radical assay [79]. The scavenging ability of eight of these compounds which are also present in this systematic review is as follows: quercetin $(70)>$ gallic acid (30) $>$ rutin $(80)>$ baicalein $(83)>$ quercitrin $(79)>$ myricitrin $(82)>$ catechin $(\mathbf{7 2})>$ luteolin $(\mathbf{7 1})$. Interestingly, $\mathbf{7 0}, \mathbf{7 1}$ and $\mathbf{8 0}$ belong to the 'best anti-inflammatory performers' of this dataset. For both 80 and 70, the $\mathrm{DPPH}$ radical scavenging activity was determined, however, only for 8o, an $\mathrm{IC}_{50}$ value was calculated which is twice as high $(9.07 \mu \mathrm{M}$ [79] versus $19.84 \mu \mathrm{M}[23]$ ) in this analysis.

In a study by Burda and Oleszek, the anti-oxidant performance of flavo-noids was determined and compounds were given an overall percentage score in anti-oxidant activity [9]. In order of score from the compounds also present in this analysis: BHT (110) $>\alpha$-tocopherol (104) $>$ quercetin $(\mathbf{7 0})>$ myricitin $(\mathbf{8 2})>$ rutin $(80)$. The highest scoring compounds are used as reference compounds in this systematic review, which is not surprising. Furthermore, 70 performs better than 8o, which is consistent with the results of this study and as described by Quideau et al. in his extensive review on polyphenols [64].

Structure-wise, it has been shown that compounds with a pyrogallol moiety are five to nine times better in $\mathrm{O}_{2}{ }^{-}$. scavenging than structures with a catechol. And as an example: myritricin (82) has an eight to nine times higher scavenging activity than quercetin (70) [27]. Both natural products were not tested for $\mathrm{O}_{2}{ }^{-}$. scavenging in this analysis, however, it is notable that the general results are the other way around: quercetin (70) is among the 'best' anti-inflammatory natural products, while myritricin (82) is among the 'worst'. The natural reference com- 
pound pyrogallol (33) though, showed strong $\mathrm{O}_{2}{ }^{-}$. scavenging [70] as can be seen in Table $S_{3}$.

Moreover, the presence of a catecholic B ring stands out in antioxidant activity [64]. In the flavonoids structural group about half of the natural products have this ring and all have reasonably high maximum inhibitory activities. In comparison, pinocembrin (64) and gnaphalinn (67) lacking the catecholic B ring, do not show any inhibition in the tested anti-oxidant assays (Table $\mathrm{S}_{3}$ ).

\subsubsection{Data collection and selection procedure}

The data collection in this systematic review has been done following a series of filtering steps as is depicted in Figure 2.1. Chosen was for English literature from two large data bases (i.e. Pubmed and Web of Knowledge), to ensure that the studies are available and understandable to a wide scientific community.

The first selection was made using the database's own search engine (for full inclusion criteria see Figure 2.1). Subsequently, further selection was done manually by screening keywords, titles and abstracts. This selection procedure has been done with the greatest care.

Notably, a large fraction of the initial search was excluded based on the fact that these papers reported effects of solvent extracts or mixtures of medicinal plants. These studies were left out since the anti-inflammatory effects of the individual components cannot be interpreted. Another very important inclusion criterion was the use of proper experimental design, including negative and positive controls, preferably in the form of a known drug. Ideally a dose-response relationship was established for the compounds. This enables a fair comparison of anti-inflammatory activity of the natural products. Unfortunately, because of this reason, a large part of the remaining articles could not be included. This is consistent with the findings described in the review of Fürst and Zündorf on natural products which made it into clinical trials [26].

In the final selection of 45 articles, the experimental data were extracted from 102 natural products (of which six were also used as a reference) and 31 synthetic reference compounds. In general, very similar assays were used to determine the anti-inflammatory potency of the natural products. In this manner, the efficacy of the natural products was compared using the maximum inhibition percentage at the corresponding concentration. Compounds that performed better than the median values were considered 'potent' (Table performance assays). The use of IC 50 values would have been the ideal way to compare the compounds, however, most studies did not determine the $\mathrm{IC}_{50}$, nor provided the data, preventing us to calculate it ourselves. Furthermore, 
the way of calculating the $\mathrm{IC}_{50}$ (absolute $v$ s relative $50 \%$ inhibition) was doubtful, making it not a straightforward comparison parameter.

This systematic review compares natural products from studies from 2000 up until July 2013. The same search was performed in August 2015, yielding 204 new studies, underlining the popularity of natural products as anti-inflammatory agents. Again, a large part of the selection was aimed at studying plant extracts and mixtures, making it impossible to elucidate the effectiveness of individual microspecies. It would therefore be a welcoming new movement to the field to focus more on the single molecules in the extracts for drug discovery and drug development.

\subsection{FUTURE PERSPECTIVES AND CONCLUSIONS}

To fully unlock the potential of natural products, well-structured research on single molecular species, with well-defined assays is recommended to enhance the collective advancements in the field. Another hurdle to be overcome is the bioavailability challenge. As can be seen in this study, many natural products have a low capacity for membrane passage. This could be addressed by using delivery systems and/or solubilizing agents. However, the existing bioavailability predictions, as described in section 2.4, are a good measure as a first step in selecting truly potent and broadly applicable natural products.

In summary, we can come to three general recommendations in order for a natural product to be a successful anti-inflammatory drug:

1. The natural product should adhere to the in section 2.4 provided bioavailability rules. A compound within these limits is more likely to be successful in inhibiting pro-inflammatory mediators. In this dataset in the group 'always performing better than median', $29 \%$ does not meet one or more of the provided bioavailability rules, compared to $44 \%$ and $41 \%$ for the 'performing at least once better than median' and 'performing always worse than median' groups (supplementary Table S9).

2. One or more sugar moieties reduces the chances to be a good antiinflammatory compound and to comply to the set oral bioavailability rules. Of the thirteen natural products with one or more sugar moieties, seven (54\%) are in the 'worst performing' group: not passing the oral bioavailability limits and with no anti-inflammatory activity. Furthermore, the sugar moiety makes it almost impossible to pass the bioavailability limits since it increases the size (hence MW and tPSA) of the compound. Arbutin (9o) is the exception, since the sugar moiety is attached to a very small 
molecule. All sugar-bearing natural products are either in the 'worst-performing' group or do exceed the limits. Rutin (80) is the only one that passes all anti-inflammatory assays, however, does not pass the bioavailability limits.

3. The reactive enol moiety is important in oxidation. 6 Most studied natural products do indeed have one or more enol moieties, often presented as a phenol. It is no surprise that these compounds perform relatively well in the oxidation assays compared to the other assays. So, from the fourteen best anti-inflammatory natural products, ten $(71 \%)$ have reactive enol moieties. The exceptions are byakangelicol (13), phyllantine (87) and phyltetralin (99).

In conclusion, present systematic review shows that phyllanthine (87), 6-shogaol (25), phyltetralin (99) and pyrogallol (33) have strong anti-inflammatory potency, whereas the majority of natural products hardly shows any anti-inflammatory activity (a full list ordered by antiinflammatory potency can be found in supplementary Table S9).

\section{Conflict of Interest}

The authors declare no competing financial interest.

Acknowledgment

NanoNextNL grant 03D.11 (Raymond M. Schiffelers).

\section{Supporting Information}

Full data tables containing all anti-inflammatory assays and outcomes of all natural products and reference compounds in this study is available here on the internet.

\section{REFERENCES}

[1] Siddig Ibrahim Abdelwahab et al. "Anti-inflammatory activities of cucurbitacin E isolated from Citrullus lanatus var. citroides: role of reactive nitrogen species and cyclooxygenase enzyme inhibition." In: Fitoterapia 82.8 (Dec. 2011), pp. 1190-1197.

[2] Cezmi A. Akdis and F. Estelle R. Simons. "Histamine receptors are hot in immunopharmacology." In: European Journal of Pharmacology 533.1-3 (2006), pp. 69-76. 
[3] K. Ashalatha et al. "Anti inflammatory potential of Decalepis hamiltonii (Wight and Arn) as evidenced by down regulation of pro inflammatory cytokines-TNF-alpha and IL-2." In: Journal of Ethnopharmacology 130.1 (July 2010), pp. 167-170.

[4] Jung Ok Ban et al. "Anti-inflammatory and arthritic effects of thiacremonone, a novel sulfur compound isolated from garlic via inhibition of NF-kappaB." In: Arthritis research E therapy 11.5 (Jan. 2009), R145.

[5] Li Bao et al. "Effects of Pithecellobium clypearia Benth extract and its main components on inflammation and allergy." In: Fitoterapia 80.6 (Sept. 2009), pp. 349-353.

[6] Liu Baolin et al. "Resveratrol inhibits the release of mediators from bone marrow-derived mouse mast cells in vitro." In: Planta medica 70.4 (Apr. 2004), pp. 305-309.

[7] Esperanza Bas et al. "Inhibition of the pro-inflammatory mediators' production and anti-inflammatory effect of the iridoid scrovalentinoside." In: Journal of ethnopharmacology 110.3 (Apr. 2007), pp. 419-427.

[8] Sekhar K. Bose et al. "Effect of bergapten from Heracleum nepalense root on production of proinflammatory cytokines." In: Natural Product Research 25.15 (Sept. 2011), pp. 1444-1449.

[9] Stanislaw Burda et al. "Antioxidant and Antiradical Activities of Flavonoids." In: Journal of Agricultural and Food Chemistry 49.6 (2001), pp. 2774-2779.

[10] J. Caldwell, I. Gardner, and N. Swales. "An introduction to drug disposition: the basic principles of absorption, distribution, metabolism, and excretion." In: Toxicologic pathology 23.2 (1995), pp. 102-114.

[11] R. Capasso et al. "Cannabidiol, extracted from Cannabis sativa, selectively inhibits inflammatory hypermotility in mice." In: British Journal of Pharmacology 154.5 (July 2008), pp. 1001-1008.

[12] C. V. Chandrasekaran et al. "In vitro modulation of LPS/calcimycin induced inflammatory and allergic mediators by pure compounds of Andrographis paniculata (King of bitters) extract." In: International Immunopharmacology 1 (), pp. 79-84.

[13] Kuo-Ping Chao et al. "Anti-inflammatory activity of sugiol, a diterpene isolated from Calocedrus formosana bark." In: Planta Medica 71.4 (Apr. 2005), pp. 300-305.

[14] Chiu-Yuan Chen et al. "Luteolin ameliorates experimental lung fibrosis both in vivo and in vitro: implications for therapy of lung fibrosis." In: Journal of agricultural and food chemistry 58.22 (Nov. 2010), pp. 11653-11661. 
[15] Yeon Sook Chi et al. "Effects of wogonin, a plant flavone from Scutellaria radix, on skin inflammation: in vivo regulation of inflammation-associated gene expression." In: Biochemical Pharmacology 66.7 (Oct. 2003), pp. 1271-1278.

[16] Kyung-Ook Chung et al. "In-vitro and in-vivo anti-inflammatory effect of oxyresveratrol from Morus alba L." In: The Journal of pharmacy and pharmacology 55.12 (Dec. 2003), pp. 1695-1700.

[17] Gabriella Corea et al. "Discovery and biological evaluation of the novel naturally occurring diterpene pepluanone as antiinflammatory agent." In: Journal of medicinal chemistry 48.22 (Nov. 2005), pp. 7055-7062.

[18] J.C. Dearden, M.T.D. Cronin, and K.L.E. Kaiser. "How not to develop a quantitative structure-activity or structure-property relationship (QSAR/QSPR)." In: SAR and QSAR in Environmental Research 20.3-4 (Apr. 2009), pp. 241-266.

[19] Christopher M. Dobson. "Chemical space and biology." In: Nature 432.7019 (2004), pp. 824-828.

[20] Wulf Dröge. "Free radicals in the physiological control of cell function." In: Physiological Reviews 82.1 (Jan. 2002), pp. 47-95.

[21] Swarnalatha Dugasani et al. "Comparative antioxidant and anti-inflammatory effects of [6]-gingerol, [8]-gingerol, [10]-gingerol and [6]-shogaol." In: Journal of ethnopharmacology 127.2 (Feb. 2010), pp. 515-520.

[22] Riyo Enomoto et al. "Wogonin Prevents GlucocorticoidInduced Thymocyte Apoptosis Without Diminishing Its Anti-inflammatory Action." In: Journal of Pharmacological Sciences 104.4 (Aug. 2007), pp. 355-365.

[23] Shih-Hua Fang, Yerra Koteswara Rao, and Yew-Min Tzeng. "Anti-oxidant and inflammatory mediator's growth inhibitory effects of compounds isolated from Phyllanthus urinaria." In: Journal of ethnopharmacology 116.2 (Mar. 2008), pp. 333-340.

[24] A. Fernandez-Arche et al. "Topical anti-inflammatory effect of tirucallol, a triterpene isolated from Euphorbia lactea latex." In: Phytomedicine 17.2 (Feb. 2010), pp. 146-148.

[25] Gerda Fouche et al. "Investigation of in vitro and in vivo antiasthmatic properties of Siphonochilus aethiopicus." In: Journal of ethnopharmacology 133.2 (Jan. 2011), pp. 843-849.

[26] Robert Fürst and Ilse Zündorf. "Plant-derived anti-inflammatory compounds: Hopes and disappointments regarding the translation of preclinical knowledge into clinical progress." In: Mediators of Inflammation 2014 (2014). 
[27] Koji Furuno, Toyokazu Akasako, and Narumi Sugihara. "The contribution of the pyrogallol moiety to the superoxide radical scavenging activity of flavonoids." In: Biological E pharmaceutical bulletin 25.1 (2002), pp. 19-23.

[28] Shayne C. Gad. "The mouse ear swelling test (MEST) in the 1990s." In: Toxicology 93.1 (1994), pp. 33-46.

[29] Hao Gao et al. "Bidesmoside triterpenoid glycosides from Stauntonia chinensis and relationship to anti-inflammation." In: Phytochemistry 70.6 (Apr. 2009), pp. 795-806.

[30] Raju Gautam and Sanjay M. Jachak. "Recent developments in anti-inflammatory natural products." In: Medicinal research reviews 29.5 (Sept. 2009), pp. 767-820.

[31] Jonathan Gershenzon and Natalia Dudareva. "The function of terpene natural products in the natural world." In: Nature chemical biology 3.7 (2007), pp. 408-414.

[32] Lian Yu Guo et al. "Anti-inflammatory effects of schisandrin isolated from the fruit of Schisandra chinensis Baill." In: European journal of pharmacology 591.1-3 (Sept. 2008), pp. 293-299.

[33] Matthew S Hayden and Sankar Ghosh. "Shared principles in NFkappaB signaling." In: Cell 132.3 (Mar. 2008), pp. 344-362.

[34] V. Hernández et al. "Anti-inflammatory profile of dehydrocostic acid, a novel sesquiterpene acid with a pharmacophoric conjugated diene." In: European journal of pharmaceutical sciences 26.2 (Oct. 2005), pp. 162-169.

[35] Yu-Chang Hou et al. "Preventive effect of silymarin in cerebral ischemia-reperfusion-induced brain injury in rats possibly through impairing NF-кB and STAT-1 activation." In: Phytomedicine 17.12 (Oct. 2010), pp. 963-973.

[36] Instant JChem Suite, v15.8.17.0. 2015. URL: http : //www. chemaxon . com.

[37] Kuo-Ching Jan et al. "Tissue distribution and elimination of estrogenic and anti-inflammatory catechol metabolites from sesaminol triglucoside in rats." In: Journal of agricultural and food chemistry 58.13 (July 2010), pp. 7693-7700.

[38] Ted W. Johnson, Klaus R. Dress, and Martin Edwards. "Using the Golden Triangle to optimize clearance and oral absorption." In: Bioorganic and Medicinal Chemistry Letters 19.19 (2009), pp. 55605564 .

[39] Kohichi Kawahara et al. "Prostaglandin E2-induced inflammation: Relevance of prostaglandin E receptors." In: Biochimica et Biophysica Acta (BBA) - Molecular and Cell Biology of Lipids 1851.4 (2015), pp. 414-421. 
[40] Sagar B. Kedare and R. P. Singh. "Genesis and development of DPPH method of antioxidant assay." In: Journal of Food Science and Technology 48.4 (2011), pp. 412-422.

[41] Olivia M. Kenny et al. "Anti-inflammatory properties of potato glycoalkaloids in stimulated Jurkat and Raw 264.7 mouse macrophages." In: Life sciences 92.13 (Apr. 2013), pp. 775-782.

[42] Inamullah Khan et al. "Anti-inflammatory activities of Taxusabietane A isolated from Taxus wallichiana Zucc." In: Fitoterapia 82.7 (Oct. 2011), pp. 1003-1007.

[43] Salman Khan et al. "Molecular mechanism of capillarisinmediated inhibition of MyD88/TIRAP inflammatory signaling in in vitro and in vivo experimental models." In: Journal of ethnopharmacology 145.2 (Jan. 2013), pp. 626-637.

[44] Riku Korhonen et al. "Nitric oxide production and signaling in inflammation." In: Current drug targets. Inflammation and allergy 4.4 (2005), pp. 471-479.

[45] Angeliki P. Kourounakis et al. "Alkannin and shikonin: effect on free radical processes and on inflammation - a preliminary pharmacochemical investigation." In: Archiv der Pharmazie 335.6 (June 2002), pp. 262-266.

[46] Christopher A. Lipinski et al. "Experimental and computational approaches to estimate solubility and permeability in drug discovery and development setting." In: Advanced Drug Delivery Reviews 46.1-3 (2001), pp. 3-26.

[47] Yue $\mathrm{Lu}$ et al. "Kirenol exerts a potent anti-arthritic effect in collagen-induced arthritis by modifying the T cells balance." In: Phytomedicine 19.10 (July 2012), pp. 882-889.

[48] Eleonora S. Mengoni et al. "Suppression of COX-2, IL-1 $\beta$ and TNF- $\alpha$ expression and leukocyte infiltration in inflamed skin by bioactive compounds from Rosmarinus officinalis L." In: Fitoterapia 82.3 (Apr. 2011), pp. 414-421.

[49] Nandipha Mnonopi et al. "The cardioprotective effects of marrubiin, a diterpenoid found in Leonotis leonurus extracts." In: Journal of ethnopharmacology 138.1 (Oct. 2011), pp. 67-75.

[50] Christopher J. Morris. "Carrageenan-Induced Paw Edema in the Rat and Mouse." In: Inflammation Protocols. Vol. 225. 2003, pp. 115-122.

[51] Ilias Muhammad et al. "Cytotoxic and antioxidant activities of alkylated benzoquinones from Maesa lanceolata." In: Phytotherapy research 17.8 (Sept. 2003), pp. 887-891. 
[52] Chanin Nantasenamat, Chartchalerm Isarankura-Na-Ayudhya, and Virapong Prachayasittikul. Advances in computational methods to predict the biological activity of compounds. July 2010.

[53] G. M. Nardi et al. "Antioxidant and anti-inflammatory effects of products from Croton celtidifolius Bailon on carrageenan-induced pleurisy in rats." In: Phytomedicine 14.2-3 (Feb. 2007), pp. 115-122.

[54] Markus F. Neurath and Susetta Finotto. "IL-6 signaling in autoimmunity, chronic inflammation and inflammation-associated cancer." In: Cytokine $\mathcal{E}$ growth factor reviews 22.2 (Apr. 2011), pp. 83-89.

[55] NIH. Pubmed. 2013. URL: http://www.ncbi.nlm.nih.gov/pubmed.

[56] Dieudonné Njamen et al. "Anti-inflammatory activity of erycristagallin, a pterocarpene from Erythrina mildbraedii." In: European journal of pharmacology 468.1 (May 2003), pp. 67-74.

[57] Se-Woong Oh et al. "Curcumin attenuates allergic airway inflammation and hyper-responsiveness in mice through NF- $\mathrm{kB}$ inhibition." In: Journal of ethnopharmacology 136.3 (July 2011), pp. 414421.

[58] Festus B. C. Okoye et al. "Anti-inflammatory and membranestabilizing stigmastane steroids from Alchornea floribunda leaves." In: Planta medica 76.2 (Feb. 2010), pp. 172-177.

[59] Olumayokun A. Olajide, Abayomi M. Ajayi, and Colin W. Wright. "Anti-inflammatory properties of cryptolepine." In: Phytotherapy research 23.10 (Oct. 2009), pp. 1421-1425.

[6o] World Health Organization. Traditional and Complementary Medicine policy. 2012, pp. 1-17.

[61] L. F. Ospina et al. "Inhibition of acute and chronic inflammatory responses by the hydroxybenzoquinonic derivative rapanone." In: Planta medica 67.9 (Dec. 2001), pp. 791-795.

[62] Katrin Palm et al. Polar molecular surface properties predict the intestinal absorption of drugs in humans. 1997.

[63] Pier Giorgio Pietta. "Flavonoids as antioxidants." In: Journal of Natural Products 63.7 (2000), pp. 1035-1042.

[64] Stéphane Quideau et al. "Plant polyphenols: chemical properties, biological activities, and synthesis." In: Angewandte Chemie (International ed. in English) 50.3 (Jan. 2011), pp. 586-621.

[65] Jie Ren, Eun Jung Han, and Sung Hyun Chung. "In Vivo andIn Vitro anti-inflammatory activities of $\alpha$-linolenic acid isolated from actinidia polygama fruits." In: Archives of Pharmacal Research 30.6 (June 2007), pp. 708-714. 
[66] Thomson Reuters. Web of Knowledge. 2013. URL: http : / / apps . webofknowledge . com / UA $\% 7 B \% 5 C$ - 7 DGeneralsearch $\%$ 7B $-\% 7 D$ input . do ? product $=U A \% 7 B \& \% 7 D$ search $\% 7 B_{-} \% 7 D m o d e=$ GeneralSearch $\% 7 B \& \% 7 D S I D=$ X2SUxzasIIAWYMpV5Fn $\% 7 B \&$ \%7DpreferencesSaved $=$.

[67] Catherine a. Rice-Evans, Nicholas J. Miller, and George Paganga. "Structure-antioxidant activity relationships of flavonoids and phenolic acids." In: Free Radical Biology and Medicine 20.7 (1996), pp. 933-956.

[68] Timothy J. Ritchie and S. J F Macdonald. "The impact of aromatic ring count on compound developability - are too many aromatic rings a liability in drug design?" In: Drug Discovery Today 14.2122 (2009), pp. 1011-1020.

[69] Céline Rivière, Alison D Pawlus, and Jean-Michel Mérillon. “Natural stilbenoids: distribution in the plant kingdom and chemotaxonomic interest in Vitaceae." In: Natural product reports 29.11 (Nov. 2012), pp. 1317-1333.

[70] Araceli Sala et al. "Assessment of the anti-inflammatory activity and free radical scavenger activity of tiliroside." In: European Journal of Pharmacology 461.1 (Feb. 2003), pp. 53-61.

[71] Flávia V. Santa-Cecília et al. "7-epiclusianone, the natural prenylated benzophenone, inhibits superoxide anions in the neutrophil respiratory burst." In: Journal of medicinal food 15.2 (Feb. 2012), pp. 200-205.

[72] Appian Subramoniam et al. "Chlorophyll revisited: antiinflammatory activities of chlorophyll A and inhibition of expression of TNF- $\alpha$ gene by the same." In: Inflammation 35.3 (June 2012), pp. 959-966.

[73] Manal Mohamed Elhassan Taha et al. "Gastroprotective activities of Turnera diffusa Willd. ex Schult. revisited: Role of arbutin." In: Journal of ethnopharmacology 141.1 (May 2012), pp. 273-281.

[74] Daniel F. Veber et al. "Molecular properties that influence the oral bioavailability of drug candidates." In: Journal of medicinal chemistry 45.12 (2002), pp. 2615-2623.

[75] Ching-Chiung Wang et al. "Inducible nitric oxide synthase inhibitors of Chinese herbs. Part 2: Naturally occurring furanocoumarins." In: Bioorganic $\mathcal{E}$ Medicinal Chemistry 8.12 (Dec. 2000), pp. 2701-2707.

[76] Michael J. Waring. "Defining optimum lipophilicity and molecular weight ranges for drug candidates-Molecular weight dependent lower $\log \mathrm{D}$ limits based on permeability." In: Bioorganic $\mathcal{E}$ Medicinal Chemistry Letters 19.10 (May 2009), pp. 2844-2851. 
[77] Han van de Waterbeemd and Eric Gifford. "ADMET in silico modelling: towards prediction paradise?" In: Nature reviews. Drug discovery 2.3 (2003), pp. 192-204.

[78] T. Yokomizo, T. Izumi, and T. Shimizu. "Leukotriene B4: metabolism and signal transduction." In: Archives of biochemistry and biophysics 385.2 (2001), pp. 231-241.

[79] Takako Yokozawa et al. "Study on the inhibitory effect of tannins and flavonoids against the 1,1-diphenyl-2-picrylhydrazyl radical." In: Biochemical Pharmacology 56.2 (1998), pp. 213-222.

[8o] Gary P. Zaloga. "Phytosterols, Lipid Administration, and Liver Disease During Parenteral Nutrition." In: Journal of parenteral and enteral nutrition (July 2015).

[81] Bin Zhang et al. "Gaultherin, a natural salicylate derivative from Gaultheria yunnanensis: towards a better non-steroidal anti-inflammatory drug." In: European journal of pharmacology 530.1-2 (Jan. 2006), pp. 166-171. 


\title{
BENCHMARKING NATURAL PRODUCTS
}

\section{Head-to-Head Comparison of Anti-Inflammatory Performance of Known Natural Products in Vitro}

Iris E. Allijn, Stefan F.C. Vaessen, Linda C. Quarles van Ufford, Kees J. Beukelman, Menno P.J. de Winther, Gert Storm, Raymond M. Schiffelers.

\begin{abstract}
Inflammation is an important therapeutic target. Due to their potency, steroidal drugs dominate the current treatment of inflammatory disorders. However, steroidal drugs can also exert a broad range of side effects and appear not always effective. This calls for the development of alternative drugs with a different mechanism of action, which are likely to be found in the field of natural products (NPs). For many NPs, strong anti-inflammatory effects have been described, but usually investigating a single compound in a single assay. In this study, eight promising NPs were selected and tested against the strong antiinflammatory drug prednisolone. For this head-to-head comparison, in vitro assays were used which represent different pathways of the inflammatory response: TNF- $\alpha$ and IL- 6 expression by macrophages, IL-8 expression by colon epithelial cells, ROS production in polymorphonuclear leukocytes and platelet activation in whole blood. Performance profiles were established which allowed us to identify curcumin, berberine chloride and epigallocatechin gallate as potential alternatives for prednisolone or other glucocorticoids in inflammation.
\end{abstract}

\subsection{INTRODUCTION}

Inflammation is an important process to defend against pathogens and injuries. A controlled acute inflammatory response is beneficial for the body. However, inflammation can become detrimental when the pro-

1 Published in PLoS ONE, 2016, 11(5), pp e0155325, DOI: 10.1371/journal.pone.0155325 
cess is dysregulated. Uncontrolled inflammation is underlying most chronic diseases such as cardiovascular disease, arthritis, asthma and type 2 diabetes mellitus [31] and is often linked to cancer development $[31,1]$. Inflammation is a complex process involving many mediators, with TNF- $\alpha$, IL- 6 , IL-8, ROS and platelet activation being key players (Figure 3.1). Even though Figure 3.1 addresses only a limited number of pathways, the complex nature of inflammation and the many mediators involved is apparent.

Five important processes in inflammation are combined into one interconnected pathway network (Figure 3.1):

TNF- $\alpha$ is part of the very extensive NF- $\kappa \beta$ pathway [6]. TNF- $\alpha$ starts multiple signaling cascades by recruiting the tumor necrosis factor receptor 1 (TNFRI), which is subsequently recruiting the TNFRI associated death domain (TRADD) [15]. TRADD on one side activates the caspase cascade which leads to apoptosis and ROS production [2]. On the other side, the core component complex IKK $\alpha / \beta$ (IK $\alpha / \beta$ kinase) of the NF- $\kappa \beta$ pathway is activated. The $\beta$ part subsequently phosphorylates I $\kappa \beta$ which in turn activates NF- $\kappa \beta$ [15]. This leads to translocation of NF- $\kappa \beta$ dimers to the nucleus and upregulation of (among others) IL6 , IL-8, TNF- $\alpha$, and manganese superoxide dismutase (Mn-SOD) $[15,2$, 14 .

IL-6 is an important activator of the Janus kinase signal transducer and an activator of transcription [5]. The JAK/Stat pathway is involved in the upregulation of pro-inflammatory cytokines in inflammation, cell proliferation and tumorigenesis $[14,5,33]$. IL-6 binds to the IL-6 receptor (IL-6R), which in turn associates with the gp130 protein complex on the cell membrane and phosphorylates JAK. Only a few cell types express the IL-6R on the cell membrane, however, all cells have a soluble form of this receptor (sIL-6R) and the gp130 dimer, meaning that JAK/Stat signaling can be activated in essentially all cell types. The complexation of IL-6 with gp130 and consequently the phosphorylation of Stat 3 is needed for a controlled inflammatory response [38, 41]. Activated Stat3 dimerizes and translocates to the nucleus, where (among others) c-Myc and c-reactive protein (CRP) are upregulated [41]. Furthermore, activated Stat 3 stimulates NF- $k \beta$ [1] and the Ras oncogene which is important in both the development of cancer $[14,5$, $33,38,41$ ] and stimulation of inflammation [14].

IL-8 is a pro-inflammatory chemokine whose expression is primarily regulated by NF- $\kappa \beta$. IL- 8 binds to G-coupled protein receptor CXCR1/2, which in turn stimulates the Ras oncogene and promotes the nuclear translocation of Stat3 [49]. It is the most powerful human neutrophil chemoattractant and stimulates tumor growth. Furthermore, TNF- $\alpha$ and ROS are potent inducers of IL-8 production [47].

At an inflammatory site, ROS (which include superoxide radicals, nitric oxide and hydrogen peroxide [2]) are produced continuously (the 


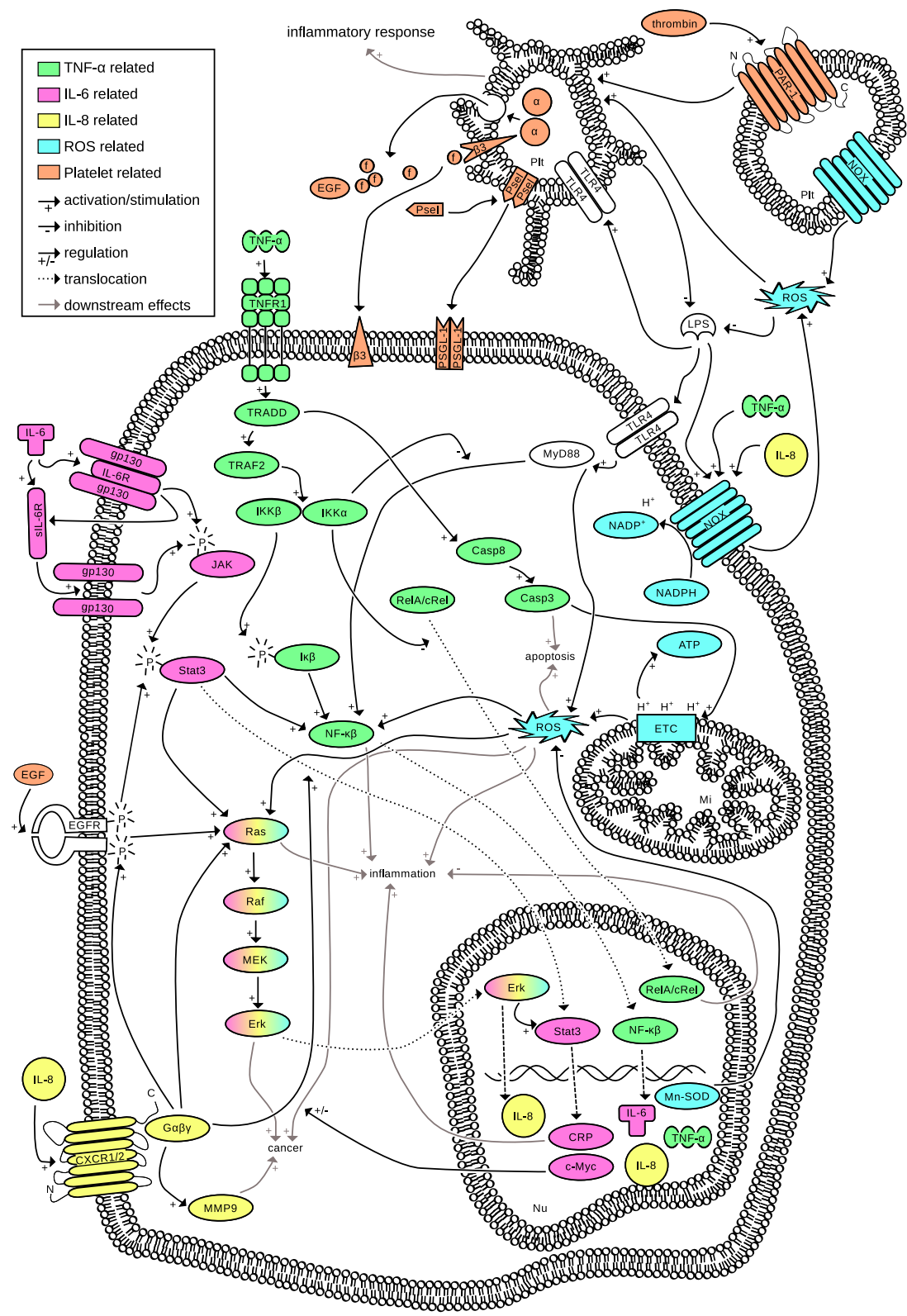

Figure 3.1: Interconnection of inflammatory pathways. Five important mediators (TNF- $\alpha$, green, IL-6, pink, IL-8, yellow, ROS, blue and Platelets, orange) of inflammation are combined into one interconnected pathway network.f $=$ fibrinogen, Psel $=$ P-selectin, EGF $=$ endothelial growth factor, $\alpha=\alpha$-granules, $\beta_{3}=\beta$-integrin receptors, $\mathrm{LPS}=$ lipopolysaccharide, $\mathrm{Plt}=$ platelet, $\mathrm{Nu}=$ nucleus. 

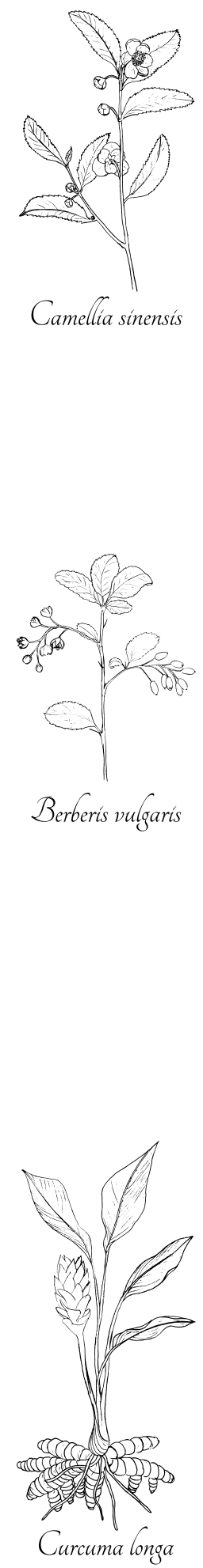

oxidative burst) as one of the first lines of attack against pathogens [9]. ROS production is vital in acute inflammation, however, a too high production of ROS can cause DNA repair failure [14] and modifications in proteins [9], and are carcinogenic [2]. Intracellularly, most ROS are produced by the mitochondrial electron transport chain (ETC), which is also stimulated in response to TNF- $\alpha$ [2]. These ROS are important for apoptosis as well as cell maintenance, but also stimulate NF- $\kappa \beta$, inflammation and cancer [9]. ROS can also activate platelets [48].

Platelets are derived from megakaryocytes, do not have a nucleus and are essential for hemostasis and thrombosis. However, platelets are also loaded with immune modulators, and can drive the inflammatory response. Platelets express NADPH oxidase (NOX) and are an important source of ROS. Upon activation by thrombin or ROS, $\alpha$-granules are secreted which contain (among others) fibrinogen, P-selectin and EGF [21].

To modulate inflammatory responses, a variety of anti-inflammatory drugs are used, which can be broadly categorized as non-steroidal anti-inflamma-tory drugs and steroids. Glucocorticoids (GCs) are the most robust anti-inflammatory agents known [50], widely used [3] and the most effective drugs in many chronic inflammatory and immune diseases $[4,10]$. In general, GCs decrease the transcription of proinflammatory cytokines and chemokines and increase the transcription of anti-inflammatory cytokines [3, 4]. In addition, there are also nongenomic actions described for GCs [50].

Despite their strong anti-inflammatory activities, a variety of systemic side effects [3] can outweigh the benefits of GC treatment [11]. About $90 \%$ of patients with chronic GC treatment develop side effects, ranging from mild (acne) to severe (Cushing Syndrome) and even life threatening events (heart disease) [10]. Besides the adverse effects, individual patients can respond differently to GCs and, with chronic use, many patients develop a form of GC resistance [50]. A small number of patients is even completely resistant to initial GC therapy [4]. The need for alternative treatments is therefore substantial [3, 4]. Particularly compounds with a different anti-inflammatory mechanism of action would be attractive. Such compounds might be found in the imminent field of natural products (NPs) because of their described anti-inflammatory bioactivity and great structural variety [12].

NPs show an enormous structural diversity $[12,8]$. They are important metabolites in plants and play key roles in antibiotic mechanisms, defense against herbivores, protection against UV radiation, nutrition and growth of the plant [36]. NPs are an important source for new drug development [12]. Indeed, half of the drugs in clinical use today is of natural origin $[12,34]$.

In this study, in order to find active but less toxic alternatives for GCs, we have selected eight NPs because of their alleged anti-inflammatory 
properties and popularity. We have compared their therapeutic in vitro efficacy with that of the GC prednisolone (PLP, Figure 3.2.1). We chose PLP, because it is an often prescribed GC which is with its intermediate potency a good representative of the GCs. As such, it serves as a good benchmark for the potency of the natural products in comparison to a strong anti-inflammatory compound. Although these eight NPs are well known, and their anti-inflammatory activity has been described, the head-to-head comparison in multiple anti-inflammatory assays, usually applied in pharmacology, is novel in phytomedicine.

Epigallocatechin gallate (EGCG, Figure 3.2.2), the main constituent in green tea leaves (Camellia sinensis) [22] has gained a lot of interest in the past decade [37]. It is a good radical scavenger with chemopreventive actions [36] and anti-inflammatory effects [37].

Berberine chloride (BBCl, Figure 3.2.3) is an alkaloid found in barberry plants (Berberis spp.) [18], which have a long history in traditional medicine $[18,35] . \mathrm{BBCl}$ is used in diabetes mellitus against insulin resistance [25]. Pharmacological properties of $\mathrm{BBCl}$ include antimicrobial, antidiarrheal [35], anti-inflammatory and anti-oxidant [12, 35, 25].

Curcumin (Cur, Figure 3.2.4) is a pigment [36] isolated from Curcuma longa which has been used in many ailments in traditional Indian medicine [24]. Cur has chemopreventive actions [36] and, despite its poor bioavailability, is used in the development of new antiinflammatory and anti-cancer drugs [12].

Apocynin (Apo, Figure 3.2.5), isolated from (Picrorhiza kurroa) [46], is well known in traditional medicine [44], and inhibits NADPH oxidase [48, 44] and platelet recruitment [48]. Apo dimerizes upon entering the cell, which enhances the effect [44]. Apocynin ester (Apo-e, Figure 3.2.6) is an ester form of apocynin making the molecule more lipophilic to increase oral bioavailability.

Paeonol (PN, Figure 3.2.7), the main phenolic compound in paeony roots (Paeonia spp.), is used in traditional medicine to treat inflammation $[17,7]$. Other reported properties of PN are anti-oxidant and apoptosis inducing effects [17].

Pterostilbene (PTS, Figure 3.2.8) is an anti-oxidant with anti-cancer properties found in berries (Vaccinium spp.) [30, 16]. PTS is an analogue of resveratrol, however with an enhanced oral bioavailability [30].

Pravastatin sodium (PSS, Figure 3.2.9) is the only included NP of microbial origin. PSS is part of the HMG-CoA reductase inhibitors, generally referred to as statins, which lower low-density lipoprotein (LDL) levels [45] and have anti-inflammatory effects in patients with cardiovascular diseases [23].

One of the difficulties in interpreting the relative potency of NPs is the fact that most studies investigate a single molecular species in a single assay. In this benchmarking study, eight NPs are tested simultaneously in four in vitro anti-inflammatory assays representing five major
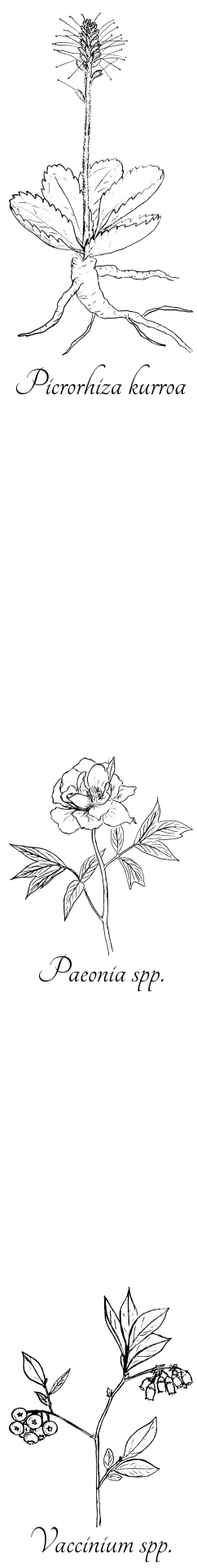
pathways of inflammation (Figure 3.1) and are compared to the reference GC PLP. Inhibition of secretion of pro-inflammatory cytokines TNF- $\alpha$ and IL- 6 by macrophages, inhibition of secretion of proinflammatory chemokine IL- 8 by colon epithelial cells, inhibition of ROS production by polymorphonuclear leukocytes and inhibition of platelet activation. From the collected results, anti-inflammatory profiles were established and compared to PLP to identify potent alternatives.

Table 3.1: Identifiers and physicochemical properties of the selected compounds. Full names, their abbreviations as used in the text and CAS registry numbers (CAS RN) are given as compound identifiers. Molecular weight (MW) and $\log \mathrm{D}$ at $\mathrm{pH} 7.4$ were predicted or calculated using Marvins' Calculater Plugins and Instant JChem 6.1.3 was used for structure database management (ChemAxon Kft. [20]). Both the MW and the $\log \mathrm{D}$ of the compounds fit within Lipinski's 'rule of 5' [26].

\begin{tabular}{lllll}
\hline Name & abbr & CAS RN & MW & $\log \mathrm{D}$ \\
\hline Prednisolone disodium phosphate & PLP & $125-02-0$ & 484.39 & -2.42 \\
Epigallocatechin gallate & EGCG & $989-51-5$ & 458.38 & 2.97 \\
Berberine chloride & BBCl & $633-65-8$ & 371.82 & -1.28 \\
Curcumin & Cur & $458-37-7$ & 368.39 & 4.12 \\
Apocynin & Apo & $498-02-2$ & 166.18 & 1.02 \\
Apocynin ester & Apo-e & $448251-47-6$ & 222.24 & 1.68 \\
Paeonol & PN & $552-41-01$ & 166.18 & 1.72 \\
Pterostilbene & PTS & $537-42-8$ & 256.30 & 3.69 \\
Pravastatin sodium & PSS & $81131-70-6$ & 446.52 & -1.38 \\
\hline
\end{tabular}

\subsection{RESULTS}

\subsubsection{Properties of Selected Compounds}

Molecular and structural properties of the selected compounds (Table 3.1 and Figure 3.2) reveal that $\mathrm{BBCl}, \mathrm{Cur}, \mathrm{PN}$ and PTS are present in their neutral form at physiological $\mathrm{pH}$ as their $\log \mathrm{P}$ and $\log \mathrm{D}$ are the same. Only PLP and PSS are negatively charged at physiological $\mathrm{pH}$, the other compounds can only be present in their cationic form at very low $\mathrm{pH}$. Furthermore, the molecular weight (MW) show that all compounds are within the limits of Lipinski's 'rule of 5' [26]. 
3.2.2 Capacity of the compounds to inhibit the production of pro-inflammatory mediators

The compounds were tested in the following anti-inflammatory assays: inhibition of secretion of pro-inflammatory cytokines TNF- $\alpha$ and IL- 6 by macrophages, inhibition of secretion of pro-inflammatory chemokine IL-8 by colon epithelial cells, inhibition of ROS production by polymorphonuclear leukocytes and inhibition of platelet activation (see Figure 3.1 for these inflammatory pathways).

Sigmoidal dose response curves were constructed as a measure of performance in all in vitro assays. For this, the following parameters were used: the maximum effect $\left(E_{\max }\right)$, the concentration at which the compound achieved $50 \%$ of the maximum effect $\left(\mathrm{IC}_{50}\right)$ and the curve fit $\left(\mathrm{R}^{2}\right)$. This means that the IC50 is not necessarily absolute $50 \%$ inhibition (Figure 3). The curve fit is an important measure for the significance of the result; 1.0 means a perfect fit, and 0.1 there is no fit, which means in our case that there is no dose dependent concentration and hence no dose response curve. Compounds which either had an $E_{\max }$ of $<30 \%$ or an $\mathrm{R}^{2}$ of $<0.70$ were considered inactive.

\subsubsection{Cell viability}

All cells in all four assays were still 100\% viable at the highest concentrations used at the end of the incubation period as determined by the

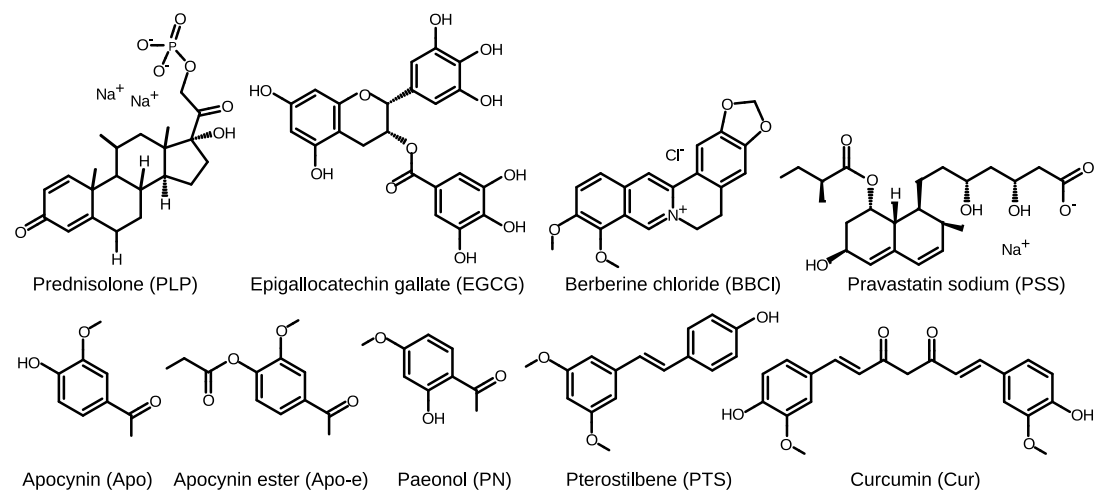

Figure 3.2: Molecular structures. The main microspecies at $\mathrm{pH} 7.4$ are depicted for the reference corticosteroid and the selected natural compounds. PLP = prednisolone disodium phosphate, EGCG = epigallocatechin gallate, $\mathrm{BBCl}=$ berberine, $\mathrm{Cur}=$ curcumin, $\mathrm{Apo}=$ apocynin, Apo-e = apocynin ester, $\mathrm{PN}=$ paeonol, $\mathrm{PTS}=$ pterostilbene, PSS = pravastatin sodium. (drawn using MarvinSketch 6.1.7, ChemAxon Kft. [20]). 
Alamar Blue assay. Also, the DMSO concentrations used to dissolve the compounds even for the highest concentrations of NPs were so low ( $\leqslant$ $0.2 \% v / v$ ) that it did not interfere with the viability of cells.

\subsubsection{Inhibition of TNF- $\alpha$ and IL-6 Secretion by Macrophages}

Inhibition by LPS stimulated murine RAW 264.7 macrophages of TNF$\alpha$ and IL- 6 secretion was quantified. Inhibition of TNF- $\alpha$ secretion was only observed in cells incubated with PLP and Cur. Although Apo-e has an $E_{\max }$ of $30 \%$, the low curve fit $\left(R^{2}=0.35\right)$ makes it a weak and unreliable inhibitor (Table 3.2). For IL-6 secretion, PLP, BBCl and Cur were inhibitory, whereas the other NPs were ineffective for both cytokines (Table 3.3). Unstimulated macrophages did not secrete TNF$\alpha$ and IL-6.

\subsubsection{Inhibition of IL-8 secretion by colon epithelial cells}

In human Caco-2 epithelial cell cultures stimulated with a pro-inflammatory cytokine mix (TNF- $\alpha$, IFN- $\gamma$ and IL- $1 \beta$ ) inhibition of IL- 8 secretion was measured. Inhibition was only achieved by the NPs EGCG (used as reference), $\mathrm{BBCl}$ and Cur. PLP and the remaining NPs did not inhibit cytokine induced IL-8 secretion by Caco-2 cells (Table 3.4).
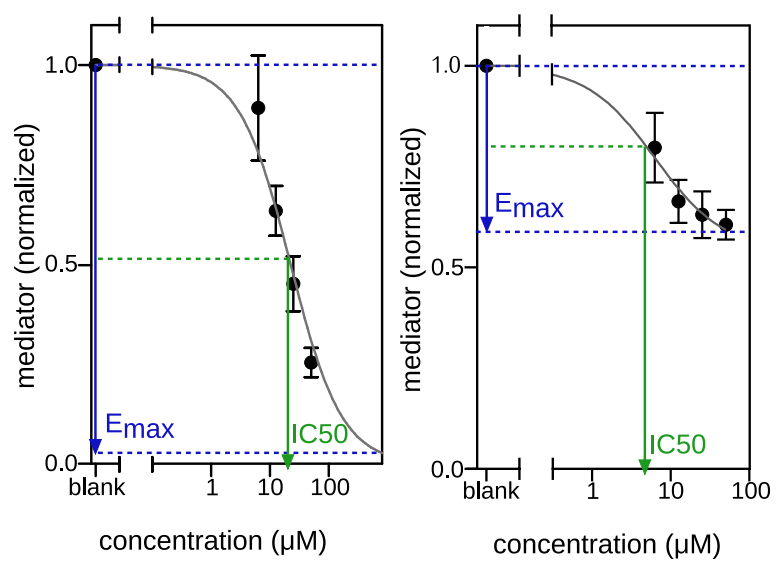

Figure 3.3: Dose response curve explanation. Two dose response curves are displayed. The first with a maximum effect $\left(E_{\max }\right)$ of close to $100 \%$ and the second with an $\mathrm{E}_{\max }$ of less than $50 \%$. The $\mathrm{IC}_{50}$ is the concentration at which $50 \%$ inhibition of the $E_{\max }$ is observed. Compounds with an $\mathrm{E}_{\max }<30 \%$ and $/$ or $\mathrm{R}^{2}<0.70$ were considered not to be inhibitory even if an $\mathrm{IC}_{50}$ could be calculated. 
Table 3.2: Inhibition of TNF- $\alpha$ secretion by RAW 264.7 macrophages. PLP and Cur demonstrated an inhibitory effect on TNF- $\alpha$ secretion by LPS stimulated RAW macrophages with $\mathrm{IC}_{50}$ s of respectively 2.6 and $7.4 \mu \mathrm{M}$. The other compounds all had an $E_{\max }$ of $<30 \%$ and were considered inactive.

\begin{tabular}{llll}
\hline Compound & $\mathrm{IC}_{50}(\mu \mathrm{M})$ & $\mathrm{E}_{\max }(\%)$ & Fit $\left(\mathrm{R}^{2}\right)$ \\
\hline PLP & 2.6 & 45 & 0.88 \\
EGCG & - & $<30$ & - \\
BBCl & - & $<30$ & - \\
Cur & 7.4 & 46 & 0.86 \\
Apo & - & $<30$ & - \\
Apo-e & 5.2 & 30 & 0.35 \\
PN & - & $<30$ & - \\
PTS & - & $<30$ & - \\
PSS & - & $<30$ & - \\
\hline
\end{tabular}

Table 3.3: Inhibition of IL-6 secretion by RAW 264.7 macrophages. PLP, BBCl and Cur demonstrated inhibitory effects on IL-6 secretion in LPS stimulated RAW macrophages with $\mathrm{IC}_{50}$ s of respectively $3.7,10.4$ and $22.5 \mu \mathrm{M}$. The other compounds all had an $E_{\max }$ of $<30 \%$ and were not considered to be inhibitory.

\begin{tabular}{llll}
\hline Compound & $\mathrm{IC}_{50}(\mu \mathrm{M})$ & $\mathrm{E}_{\max }(\%)$ & Fit $\left(\mathrm{R}^{2}\right)$ \\
\hline PLP & 3.7 & 66 & 0.97 \\
EGCG & - & $<30$ & - \\
BBCl & 10.4 & 44 & 0.77 \\
Cur & 22.5 & 100 & 0.84 \\
Apo & - & $<30$ & - \\
Apo-e & - & $<30$ & - \\
PN & - & $<30$ & - \\
PTS & - & $<30$ & - \\
PSS & - & $<30$ & - \\
\hline
\end{tabular}


3.2.6 Inhibition of ROS production in human polymorphonuclear leukocytes

ROS production in human PMNs was strongly inhibited by EGCG (used as reference), BBCl, Cur, Apo, Apo-e and PTS. PLP, PN and PSS did not have any inhibitory effect on the production of ROS (Table 3.5).

\subsubsection{Anti-platelet effects in human whole blood}

Platelet activation was determined as fibrinogen binding to platelet GP IIb/IIIa integrin receptor and P-selectin expression on the surface of plate-lets. Platelets were activated with endogenous TRAP-6. Compounds that were able to reduce fibrinogen binding were PLP, EGCG, $\mathrm{BBCl}$ and Cur. For P-selectin, $\mathrm{PLP}, \mathrm{BBCl}$ and Cur were able to reduce the expression. The remaining NPs; EGCG, Apo, Apo-e, PN, PTS and PSS, could not prevent the activation of the platelets (Table 3.6).

\subsubsection{Anti-inflammatory profiles of natural products in vitro}

Performance of each of the compounds in every assay was compared in a matrix, providing anti-inflammatory profiles (Figure 3.4). Different potencies of anti-inflammatory effect were observed based on the determined $E_{\max }, I_{50}$ and $R^{2}$ of the dose response curves. Antiinflammatory potency was classified as follows; strong inhibition ( $E_{\max }$

Table 3.4: Inhibition of IL-8 secretion by Caco-2 colon epithelial cells. EGCG, $\mathrm{BBCl}$ and Cur demonstrated inhibitory effects on IL-8 secretion in pro-inflammatory cytokine stimulated Caco-2 cells with $\mathrm{IC}_{50} \mathrm{~s}$ of respectively $78.3,15.2$ and $38.9 \mu \mathrm{M}$. The other compounds all had an $\mathrm{E}_{\max }$ of $<30 \%$ and were not considered to be inhibitory.

\begin{tabular}{llll}
\hline Compound & $\mathrm{IC}_{50}(\mu \mathrm{M})$ & $\mathrm{E}_{\mathrm{max}}(\%)$ & Fit $\left(\mathrm{R}^{2}\right)$ \\
\hline PLP & - & $<30$ & - \\
EGCG & 78.3 & 100 & 0.94 \\
BBCl & 15.2 & 52 & 0.79 \\
Cur & 38.9 & 86 & 0.76 \\
Apo & - & $<30$ & - \\
Apo-e & - & $<30$ & - \\
PN & - & $<30$ & - \\
PTS & - & $<30$ & - \\
PSS & - & $<30$ & - \\
\hline
\end{tabular}


Table 3.5: Inhibition of ROS production in human polymorphonuclear leukocytes. ROS production was not inhibited by corticosteroid PLP. Of the NPs, only PN and PSS did not inhibit ROS production. All other tested compounds showed an inhibition of ROS production with $\mathrm{E}_{\max }$ values close to $100 \%$.

\begin{tabular}{llll}
\hline Compound & $\mathrm{IC}_{50}(\mu \mathrm{M})$ & $\mathbf{E}_{\mathrm{max}}(\%)$ & Fit $\left(\mathrm{R}^{2}\right)$ \\
\hline PLP & - & $<30$ & - \\
EGCG & 5.9 & 92 & 0.91 \\
BBCl & 26.5 & 92 & 0.99 \\
Cur & 7.0 & 100 & 0.96 \\
Apo & 11.4 & 95 & 0.99 \\
Apo-e & 14.3 & 86 & 0.94 \\
PN & - & $<30$ & - \\
PTS & 22.2 & 100 & 0.83 \\
PSS & $>1000$ & 100 & 0.53 \\
\hline
\end{tabular}

Table 3.6: Inhibition of platelet activation in human whole blood. TRAP-6 induced platelet activation was determined as fibrinogen binding to platelets and PE-selectin expression on platelets. PLP, EGCG, BBCl and Cur inhibited fibrinogen binding with $\mathrm{IC}_{50} \mathrm{~s}$ of respectively 22.1, 65.9, 7.7 and 4.6 $\mu \mathrm{M}$. PLP, BBCl and Cur inhibited PE-selectin expression with $\mathrm{IC}_{50} \mathrm{~s}$ of respectively $83.2,16.5$ and $37.2 \mu \mathrm{M}$. The other compounds all had an $E_{\max }$ of $<30 \%$ and were considered to be unable to prevent platelet activation.

\begin{tabular}{lllllll}
\hline & \multicolumn{2}{l}{ Fibrinogen expression } & \multicolumn{5}{c}{ PE-selectin expression } \\
Compound & $\mathrm{IC}_{50}(\mu \mathrm{M})$ & $\mathbf{E}_{\mathrm{max}}(\%)$ & Fit $\left(\mathrm{R}^{2}\right)$ & $\mathrm{IC}_{50}(\mu \mathrm{M})$ & $\mathbf{E}_{\mathrm{max}}(\%)$ & Fit $\left(\mathrm{R}^{2}\right)$ \\
\hline PLP & 22.1 & 35 & 0.90 & 83.2 & 40 & 0.87 \\
EGCG & 65.9 & 52 & 0.84 & - & $<30$ & - \\
BBCl & 7.7 & 64 & 0.89 & 16.5 & 38 & 0.91 \\
Cur & 4.6 & 60 & 0.87 & 37.2 & 33 & 0.79 \\
Apo & - & $<30$ & - & - & $<30$ & - \\
Apo-e & - & $<30$ & - & - & $<30$ & - \\
PN & - & $<30$ & - & - & $<30$ & - \\
PTS & - & $<30$ & - & - & $<30$ & - \\
PSS & - & $<30$ & - & - & $<30$ & - \\
\hline
\end{tabular}


$>90 \%$, low $\left.\mathrm{IC}_{50}, \mathrm{R}^{2}>0.70\right)$, medium inhibition $\left(\mathrm{E}_{\max }>90 \%\right.$, medium $\mathrm{IC}_{50}, \mathrm{R}^{2}>0.70$ or $\mathrm{E}_{\max }>60 \%$, low $\mathrm{IC}_{50}, \mathrm{R}^{2}>0.70$ ), weak inhibition $\left(E_{\max }>30 \%\right.$, low $\left.\mathrm{IC}_{50}, \mathrm{R}^{2}>0.70\right)$ and no inhibitory effect $\left(E_{\max }\right.$ $\left.<30 \%, \mathrm{R}^{2}<0.70\right)$.

Cur displayed the broadest anti-inflammatory profile by showing inhibitory activity in all tests. This was followed by $\mathrm{BBCl}$, which was only unable to inhibit TNF- $\alpha$ secretion, but was inhibitory in the other assays. PLP and EGCG have contrasting profiles, only fibrinogen binding to platelets was inhibited to the same extent. Most NPs showed strong ROS production inhibition, whereas PLP was ineffective in ROS production inhibition.

\begin{tabular}{|c|c|c|c|c|c|c|c|c|c|c|}
\hline \multicolumn{2}{|c|}{ 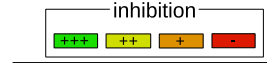 } & PLP & EGCG & $\mathrm{BBCl}$ & Cur & d compc & $\begin{array}{l}\text { nds - } \\
\text { Apo-e }\end{array}$ & PN & $\left.\right|^{\text {PTS }}$ & PSS \\
\hline TNF- $\alpha$ & $\begin{array}{l}\mathrm{IC}_{50}(\mu \mathrm{M}) \\
\mathrm{E}_{\max }(\%) \\
\text { Fit }\left(\mathrm{R}^{2}\right)\end{array}$ & \begin{tabular}{|l|}
2.6 \\
45 \\
0.9
\end{tabular} & $\begin{array}{l}- \\
<30\end{array}$ & $\begin{array}{l}- \\
<30 \\
-\end{array}$ & $\begin{array}{l}7.4 \\
46 \\
0.9\end{array}$ & $\begin{array}{l}<30 \\
-\end{array}$ & $\begin{array}{l}5.2 \\
30 \\
0.4\end{array}$ & $\begin{array}{l}- \\
<30 \\
-\end{array}$ & $\begin{array}{l}- \\
<30 \\
-\end{array}$ & $\begin{array}{l}- \\
<30 \\
-\end{array}$ \\
\hline$\frac{n}{\bar{D}}$ IL-6 & $\begin{array}{l}\mathrm{IC}_{50}(\mu \mathrm{M}) \\
\mathrm{E}_{\max }(\%) \\
\text { Fit }\left(\mathrm{R}^{2}\right)\end{array}$ & \begin{tabular}{|l|}
3.7 \\
66 \\
1.0 \\
\end{tabular} & $\begin{array}{l}- \\
<30\end{array}$ & \begin{tabular}{|l|}
10.4 \\
44 \\
0.8 \\
\end{tabular} & $\begin{array}{l}22.5 \\
100 \\
0.8 \\
\end{array}$ & $\begin{array}{l}- \\
<30 \\
-\end{array}$ & $\begin{array}{l}- \\
<30 \\
-\end{array}$ & $\begin{array}{l}- \\
<30 \\
-\end{array}$ & $\begin{array}{l}- \\
<30 \\
-\end{array}$ & $\begin{array}{l}- \\
<30 \\
-\end{array}$ \\
\hline 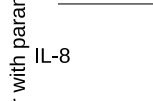 & $\begin{array}{l}\mathrm{IC}_{50}(\mu \mathrm{M}) \\
\mathrm{E}_{\max }(\%) \\
\text { Fit }\left(\mathrm{R}^{2}\right)\end{array}$ & $<30$ & $\begin{array}{l}78.3 \\
100 \\
0.9\end{array}$ & \begin{tabular}{|l|}
15.2 \\
52 \\
0.8
\end{tabular} & \begin{tabular}{|l|}
38.9 \\
86 \\
0.8
\end{tabular} & $\begin{array}{l}- \\
<30 \\
-\end{array}$ & $<30$ & $\begin{array}{l}- \\
<30 \\
-\end{array}$ & $\begin{array}{l}- \\
<30 \\
-\end{array}$ & $\begin{array}{l}- \\
<30 \\
-\end{array}$ \\
\hline 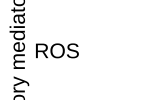 & $\begin{array}{l}\mathrm{IC}_{50}(\mu \mathrm{M}) \\
\mathrm{E}_{\max }(\%) \\
\text { Fit }\left(\mathrm{R}^{2}\right)\end{array}$ & $<30$ & $\begin{array}{l}5.9 \\
92 \\
0.9\end{array}$ & \begin{tabular}{|l|}
26.5 \\
94 \\
1.0
\end{tabular} & $\begin{array}{l}7.0 \\
100 \\
1.0\end{array}$ & $\begin{array}{l}11.4 \\
86 \\
0.9\end{array}$ & \begin{tabular}{|l|}
14.3 \\
86 \\
0.9
\end{tabular} & $\begin{array}{l}- \\
<30 \\
-\end{array}$ & $\begin{array}{l}22.2 \\
100 \\
0.8\end{array}$ & $\begin{array}{l}>1000 \\
100 \\
0.5\end{array}$ \\
\hline 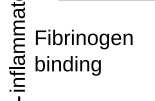 & $\begin{array}{l}\mathrm{IC}_{50}(\mu \mathrm{M}) \\
\mathrm{E}_{\max }(\%) \\
\text { Fit }\left(\mathrm{R}^{2}\right)\end{array}$ & \begin{tabular}{|l|}
22.1 \\
35 \\
0.9
\end{tabular} & $\begin{array}{l}65.9 \\
52 \\
0.8\end{array}$ & $\begin{array}{l}7.7 \\
64 \\
0.9\end{array}$ & $\begin{array}{l}4.6 \\
60 \\
0.9\end{array}$ & $\begin{array}{l}- \\
<30\end{array}$ & - & $\begin{array}{l}- \\
<30 \\
-\end{array}$ & $\begin{array}{l}- \\
<30 \\
-\end{array}$ & $\begin{array}{l}- \\
<30 \\
-\end{array}$ \\
\hline $\begin{array}{l}\text { PE-Selectin } \\
\text { expression }\end{array}$ & $\begin{array}{l}\mathrm{IC}_{50}(\mu \mathrm{M}) \\
\mathrm{E}_{\max }(\%) \\
\text { Fit }\left(\mathrm{R}^{2}\right)\end{array}$ & \begin{tabular}{|l|}
83.2 \\
40 \\
0.9 \\
\end{tabular} & $\begin{array}{l}- \\
<30 \\
-\end{array}$ & $\begin{array}{l}16.5 \\
38 \\
0.9\end{array}$ & \begin{tabular}{|l|}
37.2 \\
33 \\
0.8
\end{tabular} & $\begin{array}{l}- \\
<30 \\
-\end{array}$ & $\begin{array}{l}- \\
<30 \\
-\end{array}$ & $\begin{array}{l}- \\
<30 \\
-\end{array}$ & $\begin{array}{l}- \\
<30 \\
-\end{array}$ & $\begin{array}{l}- \\
<30 \\
-\end{array}$ \\
\hline
\end{tabular}

Figure 3.4: Anti-inflammatory profiles. Performance of the natural compounds compared to the reference corticosteroid PLP is depicted in a colored profile. Cur has an inhibitory effect in all assays, whereas PN and PSS do not induce inhibition in any of the assays. PLP is ineffective to inhibit ROS production, whereas most NPs are very effective. (strong inhibition, green) $E_{\max }>90 \%$, low $\mathrm{IC}_{50}, \mathrm{R}^{2}$ $>0.70$. (medium inhibition, yellow) $\mathrm{E}_{\max }>90 \%$, medium $\mathrm{IC}_{50}$, $\mathrm{R}^{2}>0.70$ or $\mathrm{E}_{\max }>60 \%$, low $\mathrm{IC}_{50}, \mathrm{R}^{2}>0.70$. (weak inhibition, orange) $E_{\max }>30 \%$, low $\mathrm{IC}_{50}, \mathrm{R}^{2}>0.70$. (no inhibition, red) $\mathrm{E}_{\max }<30 \%, \mathrm{R}^{2}<0.70$. PLP $=$ prednisolone disodium phosphate, EGCG = epigallocatechin gallate, $\mathrm{BBCl}=$ berberine chloride, $\mathrm{Cur}=$ curcumin, Apo = apocynin, Apo-e = apocynin ester, $\mathrm{PN}=$ paeonol, PTS $=$ pterostilbene, PSS $=$ pravastatin sodium . 


\section{$3 \cdot 3$ DISCUSSION}

Inflammation is at the foundation of most chronic diseases and thus an important target. GCs are very potent and robust anti-inflammatory agents and widely used to treat inflammation [16], however they possess a broad range of adverse effects [3].

In this study, we have performed an in vitro head-to-head performance comparison of eight NPs and prednisolone to identify the most potent NP and best GC alternative. The anti-inflammatory performance of each of the tested NPs has already been described before, however, the approach of simultaneously testing multiple compounds in multiple assays, which is common practice in pharmaceutical research, is novel in phytotherapy research. This way of testing and comparison allows ordering of the (anti-inflammatory) activity and more important the potency of NPs. In order to accomplish this, we have used five key parameters of inflammation, i.e. inhibition of TNF- $\alpha$, IL-6 and IL- 8 expression, ROS production and platelet activation (as set out in Figure 3.1). We have chosen for in vitro comparison only since the complexity of in vivo models is high and makes interpretation of inhibition of multiple inflammatory pathways difficult.

Interestingly, our reference GC PLP, which is one of the most potent anti-inflammatory drugs, does not affect all five pathways. The observed in vitro inhibition of TNF- $\alpha$ and IL- 6 is consistent with what is described in literature, with prednisolone inhibiting TNF- $\alpha$ secretion by macrophages [13] and lymphocytes [28] and both TNF- $\alpha$ and IL-6 secretion by monocytes [29]. The observed result of PLP to affect platelet function is consistent with what has been described before [32, 27]. Interestingly, both IL-8 secretion and ROS production were not inhibited by PLP in our experiments. In literature the inhibition of IL-8 secretion by nasal epithelial cells [39] and inhibition of ROS production in platelets [40] is described, however both studies used different cell lines and stimuli than we used in our assays. The profile of PLP gives us room to select NPs with a similar or contrasting profile, addressing different pathways of inflammation and ideally resulting in less side effects.

Most of the tested NPs, except for PN and PSS, are strong ROS production inhibitors, which is their most important property described in literature [36]. However, for half of them, this is also the only inflammatory pathway they affect and such a narrow profile is unlikely to be competing with that of PLP.

Nonetheless, three of the tested NPs, namely Cur, BBCl and EGCG, do show interesting anti-inflammatory profiles. Cur truly stands out with a stronger and broader anti-inflammatory profile than PLP, inhibiting all tested parameters. This broad profile is consistent with the

Please see chapter 5 for curcumin and chapter 6 and 7 for berberine. 
ability of Cur to combat numerous inflammatory diseases via multiple pathways [43]. This makes Cur a promising alternative for GCs like PLP, even the more since PLP is the only GC to inhibit platelet activation so far [27], making the anti-inflammatory profile for GCs as a class even narrower. The broad profile of Cur is followed by $\mathrm{BBCl}$, which is only ineffective in inhibiting TNF- $\alpha$ secretion. Another attractive NP is EGCG, which has a positive performance on the same number of parameters as PLP, addressing the opposite inflammatory pathways except for platelet activation inhibition.

In conclusion, the broader or contrasting profile of the NPs curcumin, berberine and epigallocatechin gallate makes these NPs possible attractive alternatives for GCs like prednisolone disodium phosphate. Because of the poor bioavailability and predominantly hydrophobic nature of these NPs, solubilizers are indispensable. Drug delivery systems are therefore a promising approach to enhance the bioavailability and boost the potency of curcumin, berberine and epigallocatechin gallate as anti-inflammatory compounds.

\subsection{MATERIALS \& METHODS}

\subsubsection{Ethical statements}

Blood was collected from anonymous healthy human volunteers. All volunteers have provided written informed consent prior to the first donation. The medical ethical board of UMC Utrecht (Utrecht, The Netherlands) and/or Sanquin Blood Supply Foundation (Amsterdam, The Netherlands) provided approval for the experimental protocols.

\subsection{2 chemicals}

Berberine chloride form, Lipopolysaccharides from Escherichia coli 055:B5, Penicillin - Streptomycin, Hanks' Balanced Salts, Zymosan A from Saccharomyces cerevisia, luminol, resazurin sodium salt, sulfuric acid, magnesium sulfate, potassium chloride and formaldehyde were purchased from Sigma-Aldrich Chemie BV, The Netherlands. Prednisolone disodium phosphate was obtained from Fagron BV, The Netherlands. Apocynin, apocynin-ester and paeonol were provided by PhytoGenix BV, The Netherlands. Pterostilbene was purchased from Chromadex Inc., USA, pravastatin sodium from AK Scientific Inc, USA and epigallocatechin gallate and curcumin from Chengdu Biopurity Phytochemicals Ltd. Dimethyl sulfoxide, albumin bovine fraction V and 1-step ultra TMB ELISA substrate were obtained from Thermo Scientific BV, The Netherlands. Tween 20 and HEPES were purchased 
from Acros Organics, Belgium. TNF- $\alpha$ and IL- 6 cytosets were acquired from Life Technologies BV, The Netherlands, IL-8 cytoset from R\&D Systems, Minneapolis, Minnesota, USA and RPMI-1640 without L-glutamine and L-glutamine $200 \mathrm{mM}$ from PAA Laboratories $\mathrm{GmbH}$, Germany. Fetal bovine serum (FBS) was purchased from Lonza, Belgium, heparine from Leo Pharmaceuticals, Denmark, gelatin from Genfarma, Spain and Percoll from Amersham Pharmacia. Ammonium chloride, potassium bicarbonate and sodium chloride were obtained from Merck BV, The Netherlands. Ethylenediaminetetraacetic acid and sodium bicarbonate were purchased from Baker Inc., USA. TRAP-6 was acquired at Bachem USA. Fibrinogen/FITC polyclonal rabbit anti-human was purchased from DAKO, Denmark and PE mouse/anti-human CD62P from BD Biosciences, USA. Cypridina luciferin analog was obtained from TCI N.V. Europe. TNF- $\alpha$, IFN- $\gamma$ and IL- $1 \beta$ were purchased from Immunotools, Friesoythe, Germany. Minimum Essential Medium, nonessential amino acids and sodium pyruvate were acquired from Gibco, Paisly, Scotland.

\subsubsection{Incubation of RAW 264.7 cells with natural products and PLP}

RAW 264.7 macrophages were a gift from the Department of Medical Biochemistry at the Academic Medical Center, Amsterdam, The Netherlands. Cells were cultured in RPMI-1640 medium substituted with 10\% FBS, $2 \mathrm{mM}$ penicillin/streptomycin and $2 \mathrm{mM}$ L-glutamine. Cells were split twice a week until maximum passage number 32 .

$10,000 \mu \mathrm{M}$ stock solutions of the NPs were prepared one day prior to the assay. For $\mathrm{BBCl}$, a stock solution of $5000 \mu \mathrm{M}$ was used. All compounds except PLP were first dissolved in DMSO, keeping a DMSO concentration of $\leqslant 0.2 \%$ for use in the cell culture. Subsequently, the compounds were dissolved to the desired stock concentration using milliQ water.

Cells were seeded into 96-well cell culture plates at a concentration of $5^{*} 10^{5}$ cells $/ \mathrm{mL}$ and left to settle at $37^{\circ} \mathrm{Cin}$ a humidified incubator containing $5 \% \mathrm{CO}_{2}$. After $6-8 \mathrm{~h}$, the cells were tightly adhered to the bottom of the wells. We have chosen the cell density to allow the cells to reach confluency over the course of the experiment. Less dense seeding of the macrophages resulted in poor and uneven growth of the cell monolayer. The medium was taken off, and concentrations between 4 and $250 \mu \mathrm{M}$ twice in triplicate per compound were added to the cells and incubated for $2 \mathrm{~h}$ in the same incubator. Incubation with medium only was used as a negative control and incubation with PLP served as a positive control. Subsequently, the compound containing medium was taken off and cells were incubated for $12 \mathrm{~h}$ with $250 \mathrm{ng} / \mathrm{mL}$ LPS to induce an inflammatory response. 
To properly establish the incubation times in the RAW 264.7 macrophages, first we established the incubation and stimulation order with different LPS stimulation times. First sample incubation followed by 12 $h$ LPS stimulation proved best for a dose response effect. Preconditioning of the cells to an anti-inflammatory state by the chosen compounds, produces a far more reproducible halt on the LPS stimulatory response, that is in line with the described pharmacological activities of antiinflammatory compounds. In contrast, LPS stimulation first produces an almost instantaneous activation that is difficult to control at meaningful drug concentrations with a strong dependence of the timing of the intervention.

Only one set of triplicates received LPS, the other set got plain medium instead and served as a negative (unstimulated) control. After LPS stimulation, supernatant was used for cytokine level determination using an ELISA assay (see section 3.4.5).

Cells were subsequently checked for their viability using Alamar blue solution (440 $\mu \mathrm{M}$ resazurin salt in PBS). Medium containing Alamar blue (1: $10 \mathrm{v} / \mathrm{v}$ medium/Alamar blue) was added to the cells and after 3 to $4 \mathrm{~h}$ incubation at $37^{\circ} \mathrm{C}$, fluorescence was measured at excitation/emission $560 / 590 \mathrm{~nm}$ using a spectrophotometer and compared to untreated cells. The experiment was repeated three times $(n=3)$.

\subsubsection{Incubation of Caco-2 cells with natural products and PLP}

The human colon carcinoma cell line, Caco-2, was obtained from the German Collection of Microorganisms and Cell Cultures (DSMZ ACC 169, Braunschweig, Germany). Caco-2 cells were cultured in MEM, supplemented with $1 \%(v / v)$ non-essential amino acids, $1 \mathrm{M}$ sodium pyruvate, $50 \mathrm{U} / \mathrm{mL}$ streptomycin, $50 \mathrm{U} / \mathrm{mL}$ penicillin and $10 \%(\mathrm{v} / \mathrm{v})$ heatinactivated FBS. Cells were grown at $37{ }^{\circ} \mathrm{Cin}$ a humidified incubator containing $5 \% \mathrm{CO}_{2}$.

For experiments, Caco-2 cells were seeded in 96-wells cell culture plates at 10,000 cells/well and allowed to grow to confluence. After four days, cells were incubated with the compounds at $37{ }^{\circ} \mathrm{Cin}$ a humidified incubator for $2 \mathrm{~h}$. Afterwards, a pro-inflammatory cytokine mix of TNF- $\alpha$, IFN- $\gamma$ and IL- $1 \beta$ (final concentrations 10,5 and $1 \mathrm{ng} / \mathrm{mL}$, respectively) was added and cells were incubated another $16 \mathrm{~h}$ at 37 ${ }^{\circ} \mathrm{Cin}$ the same incubator. After $16 \mathrm{~h}$, supernatant was collected and stored at $-80{ }^{\circ} \mathrm{C}$ for subsequent determination of IL-8 concentrations using a commercial ELISA (R\&D Systems, Minneapolis, Minnesota, USA). After aspirating the supernatant, cell viability was checked using Alamar blue (see section 3.4.3). The experiment was repeated three times $(n=3)$. 


\subsubsection{TNF- $\alpha$, IL-6 and IL-8 ELISA}

ELISAs were performed as per suppliers instructions with some minor modifications. Briefly, immuno Maxisorp plates (Nunc art NO. 439454) were coated with coating antibody in PBS, sealed and left to incubate over night at room temperature (RT). Between each step, wells were washed using $0.05 \%$ Tween 20 in PBS. After coating, cells were blocked for at least $1.5 \mathrm{~h}$ using block buffer (0.5\% BSA in PBS for TNF- $\alpha$ and IL-6 and $1 \%$ BSA with $0.05 \% \mathrm{NaN}_{3}$ in PBS for IL-8).

First incubation: wells were either incubated with standards (0-1000 $\mathrm{pg} / \mathrm{mL}$ for TNF- $\alpha$ and IL-6 and $0-2000 \mathrm{pg} / \mathrm{mL}$ for IL-8) in $1: 2$ serial dilutions in assay diluent $(0.5 \%$ BSA and $5 \%$ FBS in PBS for TNF- $\alpha$ and IL- 6 and $0.1 \%$ BSA, $0.05 \%$ Tween 20 in Tris-buffered Saline for IL8 ) in duplicates or with samples (supernatant of incubation assay) in triplicates for $1 \mathrm{~h}$ (TNF- $\alpha$ and IL-6) or $2 \mathrm{~h}$ (IL-8) at RT in the dark. Second incubation: cells were incubated with detection antibody in block buffer for $1 \mathrm{~h}$ (TNF- $\alpha$ and IL-6) or $2 \mathrm{~h}$ (IL-8) at RT in the dark. Third incubation: wells were incubated with streptavidin-HRP solution in block buffer for $1 \mathrm{~h}$ at RT in the dark.

Plates were developed by adding 1-step ULTRA TMB ELISA substrate and the reaction was stopped after all concentrations of the standard showed coloration, with the same volume of Stop Solution $\left(\mathrm{H}_{2} \mathrm{SO}_{4}, 1.8 \mathrm{M}\right.$ for TNF- $\alpha$ and IL-6 and $1 \mathrm{M}$ for IL-8). Optical density was measured at $450 \mathrm{~nm}$ using a spectrophotometer.

\subsubsection{Isolation of polymorphonuclear leukocytes from human buffy coats}

For the isolation of polymorphonuclear leukocytes (PMNs), human buffy coats (Sanquin Noordwest, Amsterdam, The Netherlands) were poured into $15 \mathrm{~mL}$ PBS/heparin solution (10 IE heparin/mL PBS) in a $50 \mathrm{~mL}$ tube and mixed carefully. $12 \mathrm{~mL}$ Percoll solution $(0.15 \mathrm{M} \mathrm{NaCl}$ in $1: 1.6$ Percoll $\mathrm{diH}_{2} \mathrm{O}$ ) was put under the buffy without disturbing the forming gradient. Tubes were spun at RT at $1200 \mathrm{~g}$ for $20 \mathrm{~min}$ without break. The top layer containing plasma, mononuclear cells and Percoll was taken off and discarded, leaving the pellet consisting of erythrocytes and PMNs undisturbed. Subsequently, cold lysis buffer $(0.16$ $\mathrm{M} \mathrm{NH}{ }_{4} \mathrm{Cl}, 10 \mathrm{mM} \mathrm{KHCO} 3$ and $0.1 \mathrm{mM} \mathrm{Na} \mathrm{KaDTA}_{2}$ in $\mathrm{diH}_{2} \mathrm{O}$ ) was added, the tubes swirled carefully and kept on ice until the solution was blackish red. Tubes were spun at $400 \mathrm{~g}$ for $5 \mathrm{~min}$ with break at RT and the supernatant containing the lysed erythrocytes was taken off and discarded. This step was repeated to remove as many erythrocytes as possible. The pellet containing mostly PMNs, was taken up in $10 \mathrm{~mL}$ HBSS-gel (4.2 mM NaHCO 3 in Hank's Buffered Salt Solution (HBSS) substituted with $1 \%$ gelatin solution in $\mathrm{diH}_{2} \mathrm{O}$ ) and the cell concentra- 
tion was determined using Türk solution and a hemocytometer. PMNs were diluted until approximately $1 * 10^{7}$ cells/mL HBSS-gel and were ready to use (see section 3.4.7).

\subsubsection{Oxidative burst assay}

All compounds except PLP were dissolved in DMSO and further diluted using HBSS buffer to the desired stock concentrations. These concentrations were a 4-fold of the highest of the duplicate $1: 2$ serial dilution series of the compounds: $4000 \mu \mathrm{M}$ for PLP, PN and PSS, $1000 \mu \mathrm{M}$ for BBCl, Apo, Apo-e and PTS, $200 \mu \mathrm{M}$ for EGCG and Cur and $1 \%$ for the DMSO control.

EGCG was used as a positive control and HBSS-gel as blank. $50 \mu \mathrm{L}$ of standards was pipetted in duplicates into white 96-well plates. To this, $50 \mu \mathrm{L}$ luminol was added as an enhancer of the luminescence and $50 \mu \mathrm{L}$ PMNs were added. At last, $50 \mu \mathrm{L}$ human serum opsonized zymosan was added to start the reaction and luminescence was immediately measured for $30 \mathrm{~min}$ at $3 \mathrm{~min}$ intervals at $37^{\circ} \mathrm{C}$ (Titertek Luminoskan, TechGen International, Zellik, Belgium). Inhibition of ROS production compared to the blank was measured. The assay was repeated for four different donors $(n=4)$.

\subsubsection{Platelet activation assay in whole blood}

In this assay, PE-anti-P-selectin and FITC-anti-fibrinogen were added to drug stock concentrations made in HBS buffer (10 mM HEPES, 150 $\mathrm{mM} \mathrm{NaCl}, 1 \mathrm{mM} \mathrm{MgSO}_{4}$ and $5 \mathrm{mM} \mathrm{KCl}$ ) at $\mathrm{pH}$ 7.4. Blood from healthy human volunteers was gently mixed with compound solutions at specific concentrations at a ratio of $1: 10(v / v$ buffer/blood $)$ and were incubated $30 \mathrm{~min}$ at RT. Subsequently $60 \mu \mathrm{M}$ of the platelet activator Thrombin Receptor Activator Peptide 6 (TRAP- 6) was added. Fixative solution (154 mM NaCl and $0.2 \%$ formaldehyde) was added to samples taken after 1, 10 and $20 \mathrm{~min}$, with a sample to fixative solution ratio of $1: 20(v / v)$. Fluorescence intensity of P-selectin (PE) and fibrinogen (FITC) was measured by flow cytometry with a FACS Canto II apparatus (BD Biosciences, San Jose, CA, USA) and analyzed with FACSDivaTM software (BD Biosciences, San Jose, CA, USA). 10,000 events were recorded per sample. The assay was repeated for three different donors $(n=3)$. The area under the curve (AUC) was calculated to determine the extent of platelet activation. 


\subsubsection{Scientific calculations}

Graphs and calculations were made using GraphPad Prism (GraphPad Prism version 5.o for Windows, GraphPad Software, Inc [19]). IC 50 s for the assay parameters (i.e. TNF- $\alpha$, IL-6, IL- 8, ROS and platelets) were calculated using the 'log(inhibitor) vs. response' of the 'non-linear regression of single data' analysis function. The curve was fit following the 'least squares (ordinary) fit', the top of the curve was set to constant equal to 1.0 (to which the data was normalized). Each replicate of $\mathrm{Y}$ was considered as an individual point and the curve was fit with a maximum of 1000 iterations and $\mathrm{IC}_{50} \mathrm{~s}$ with $\mathrm{R}^{2}$ and span (as maximum inhibitory effect, $E_{\max }$ ) were used. For the RAW 264.7 and the Caco-2 cell cultures, data of three separate experiments were used $(n=3)$, PMNs of four donors $(n=4)$ and whole blood from three donors $(n=3)$.

\subsubsection{Molecular structures and property calculation}

Chemical structures and CAS registry numbers were resolved using SciFinder [42]. Structures were drawn using MarvinSketch 6.1.5, properties were predicted and calculated using Marvins' Calculater Plugins. Instant JChem 6.1.3 was used for structure database management (all from ChemAxon Kft. [20]).

\section{Competing interest}

KJB is an employee and shareholder of PhytoGenix BV. This does not alter the authors' adherence to PLOS ONE policies on sharing data and materials.

\section{Author contributions}

Conceived and designed the experiments: IEA RMS MPJW SFCV LCQ. Performed the experiments: IEA SFCV RMS. Analyzed the data: IEA. Contributed reagents/materials/analysis tools: KJB MPJW. Wrote the paper: IEA RMS GS. Advice: KJB GS.

\section{Funding}

This work was supported by Stichting Innovatie Alliantie, RAAK PRO2-oo1 NAT-TEST, (www.regieorgaan-sia.nl) to SFCV; Stichting Techniek en Wetenschap - NanoNextNL 03D.11, (www.nanonextnl.nl) to RMS. PhytoGenix BV provided support of salary to KJB, who is also a shareholder. The specific roles of these authors are articulated in the 'author contributions' section. The funders had no role in the study design, 
data collection an analysis, decision to publish, or preparation of the manuscript.

\section{REFERENCES}

[1] Toru Atsumi et al. "Inflammation amplifier, a new paradigm in cancer biology." In: Cancer research 74.1 (Jan. 2014), pp. 8-14.

[2] Yun Soo Bae et al. Regulation of reactive oxygen species generation in cell signaling. Dec. 2011.

[3] Peter J. Barnes. "Anti-inflammatory actions of glucocorticoids: molecular mechanisms." In: Clinical science (London, England : 1979) 94.6 (June 1998), pp. 557-572.

[4] Peter J. Barnes. "Glucocorticosteroids: current and future directions." In: British journal of pharmacology 163.1 (May 2011), pp. 2943 .

[5] Cellsignal.com. Jak / Stat Signaling : IL-6 Receptor Family. 2014. URL: http : / / wWw . cellsignal . com / common / content / content . jsp?id=pathways $-i l 6$.

[6] Cellsignal.com. NF-K $\beta$ Signaling. 2014. URL: http : / / WwW . cellsignal . com / common / content/content . jsp ? id=pathways nfkb.

[7] Na Chen et al. "Paeonol suppresses lipopolysaccharide-induced inflammatory cytokines in macrophage cells and protects mice from lethal endotoxin shock." In: Fundamental $\mathcal{E}$ clinical pharmacology 28.3 (June 2014), pp. 268-276.

[8] Young-Won Chin et al. "Drug discovery from natural sources." In: The AAPS journal 8.2 (Jan. 2006), E239-E253.

[9] Wulf Dröge. "Free radicals in the physiological control of cell function." In: Physiological Reviews 82.1 (Jan. 2002), pp. 47-95.

[10] William Ericson-Neilsen and AD Kaye. "Steroids: Pharmacology, Complications, and Practice Delivery Issues." In: The Ochsner Journal 14.2 (Jan. 2014), pp. 203-207.

[11] L. Fardet et al. "Corticosteroid-induced clinical adverse events: Frequency, risk factors and patient's opinion." In: British Journal of Dermatology 157.1 (July 2007), pp. 142-148.

[12] Raju Gautam and Sanjay M. Jachak. "Recent developments in anti-inflammatory natural products." In: Medicinal research reviews 29.5 (Sept. 2009), pp. 767-820. 
[13] Domokos Gerö et al. "Identification of pharmacological modulators of HMGB1-induced inflammatory response by cell-based screening." In: PloS ONE 8.6 (Jan. 2013), e65994.

[14] Emine Guven Maiorov et al. "The structural network of inflammation and cancer: merits and challenges." In: Seminars in cancer biology 23.4 (Aug. 2013), pp. 243-251.

[15] Matthew S Hayden and Sankar Ghosh. "Shared principles in NFkappaB signaling." In: Cell 132.3 (Mar. 2008), pp. 344-362.

[16] Chin-Lin Lin Hsu et al. "The inhibitory effect of pterostilbene on inflammatory responses during the interaction of $3 \mathrm{~T}_{3}-\mathrm{L}_{1}$ adipocytes and RAW 264.7 macrophages." In: Journal of agricultural and food chemistry 61.3 (Jan. 2013), pp. 602-610.

[17] H. Huang et al. "A genome-wide microarray analysis reveals anti-inflammatory target genes of paeonol in macrophages." In: Inflammation research 57.4 (Apr. 2008), pp. 189-198.

[18] M. Ikram. "A review on the chemical and pharmacological aspects of genus Berberis." In: Planta medica 28.4 (1975), pp. 353358.

[19] GraphPad Software Inc. GraphPad Prism. 2014. URL: http://www. graphpad.com/scientific-software/prism/.

[20] Instant JChem Suite, v15.8.17.o. 2015. URL: http: //Www. chemaxon . com.

[21] C. N. Jenne, R. Urrutia, and P. Kubes. "Platelets: bridging hemostasis, inflammation, and immunity." In: International journal of laboratory hematology 35.3 (June 2013), pp. 254-261.

[22] T. Kada et al. "Detection and chemical identification of natural bio-antimutagens. A case of the green tea factor." In: Mutation research 150.1-2 (1985), pp. 127-132.

[23] Vera Krane and Christoph Wanner. "Statins, inflammation and kidney disease." In: Nature reviews. Nephrology $7 \cdot 7$ (July 2011), pp. 385-397.

[24] Ramadasan Kuttan et al. "Potential anticancer activity of turmeric (Curcuma longa)." In: Cancer letters 29.2 (1985), pp. 197-202.

[25] Zheng Li et al. "Antioxidant and Anti-Inflammatory Activities of Berberine in the Treatment of Diabetes Mellitus." In: Evidence-based complementary and alternative medicine : eCAM 2014 (Jan. 2014), p. ID 289264. 
[26] Christopher A. Lipinski et al. "Experimental and computational approaches to estimate solubility and permeability in drug discovery and development setting." In: Advanced Drug Delivery Reviews 46.1-3 (2001), pp. 3-26.

[27] Elisabetta Liverani et al. "Prednisolone exerts exquisite inhibitory properties on platelet functions." In: Biochemical pharmacology 83.10 (May 2012), pp. 1364-1373.

[28] G.S. Madretsma et al. "Nicotine inhibits the in vitro production of interleukin 2 and tumour necrosis factor- $\alpha$ by human mononuclear cells." In: Immunopharmacology 35.1 (Oct. 1996), pp. 47-51.

[29] Henrik Rasmus Maltesen et al. "Methylprednisolone prevents tumour necrosis factor-alpha-dependent multinucleated giant cell formation." In: Rheumatology 49.11 (Nov. 2010), pp. 2037-2042.

[30] Denise McCormack and David McFadden. "Pterostilbene and cancer: current review." In: The Journal of surgical research 173.2 (Apr. 2012), e53-e61.

[31] Ruslan Medzhitov. "Origin and physiological roles of inflammation." In: Nature 454.7203 (July 2008), pp. 428-35.

[32] Leonardo A. Moraes et al. "Ligand-specific glucocorticoid receptor activation in human platelets." In: Blood 106.13 (Dec. 2005), pp. 4167-4175.

[33] Markus F. Neurath and Susetta Finotto. "IL-6 signaling in autoimmunity, chronic inflammation and inflammation-associated cancer." In: Cytokine $\mathcal{E}$ growth factor reviews 22.2 (Apr. 2011), pp. 83-89.

[34] Ian Paterson and Edward a Anderson. "Chemistry. The renaissance of natural products as drug candidates." In: Science 310.5747 (Oct. 2005), pp. 451-453.

[35] Gonçalo C. Pereira et al. "Mitochondrially targeted effects of berberine [Natural Yellow 18, 5,6-dihydro-9,10dimethoxybenzo(g)-1,3-benzodioxolo(5,6-a) quinolizinium] on K1735-M2 mouse melanoma cells: comparison with direct effects on isolated mitochondrial fractions." In: The Journal of pharmacology and experimental therapeutics 323.2 (Nov. 2007), pp. 636-649.

[36] Stéphane Quideau et al. "Plant polyphenols: chemical properties, biological activities, and synthesis." In: Angewandte Chemie (International ed. in English) 50.3 (Jan. 2011), pp. 586-621.

[37] Sharayah Riegsecker et al. "Potential benefits of green tea polyphenol EGCG in the prevention and treatment of vascular inflammation in rheumatoid arthritis." In: Life sciences 93.8 (Sept. 2013), pp. 307-312. 
[38] Stefan Rose-John. "IL-6 trans-signaling via the soluble IL-6 receptor: importance for the pro-inflammatory activities of IL-6." In: International journal of biological sciences 8.9 (ja 2012), pp. 12371247.

[39] F. Sachse et al. "Anti-inflammatory effects of ciprofloxacin in S. aureus Newman induced nasal inflammation in vitro." In: Journal of inflammation 5 (Jan. 2008), p. 11.

[40] Bernd M. Sanner et al. "Effects of glucocorticoids on generation of reactive oxygen species in platelets." In: Steroids 67.8 (July 2002), pp. 715-719.

[41] Pasquale Sansone and Jacqueline Bromberg. "Targeting the interleukin-6/Jak/stat pathway in human malignancies." In: Journal of clinical oncology : official journal of the American Society of Clinical Oncology 30.9 (Mar. 2012), pp. 1005-1014.

[42] Chemical Abstract Service. SciFinder. 2013. URL: https : //scifinder. cas.org.

[43] Adeeb Shehzad, Gauhar Rehman, and Young Sup Lee. "Curcumin in inflammatory diseases." In: 39.1 (2013), pp. 69-77.

[44] J. Stefanska and R. Pawliczak. "Apocynin: molecular aptitudes." In: Mediators of inflammation 2008 (Jan. 2008), p. 106507.

[45] Jonathan A. Tobert. "Lovastatin and beyond: the history of the HMG-CoA reductase inhibitors." In: Nature reviews. Drug discovery 2.7 (July 2003), pp. 517-526.

[46] Edwin Van den Worm et al. "Effects of methoxylation of apocynin and analogs on the inhibition of reactive oxygen species production by stimulated human neutrophils." In: European Journal of Pharmacology 433.2-3 (Dec. 2001), pp. 225-230.

[47] Hannelien Verbeke et al. "The role of CXC chemokines in the transition of chronic inflammation to esophageal and gastric cancer." In: Biochimica etBbiophysica Acta 1825.1 (Jan. 2012), pp. 117129.

[48] Francesco Violi and Pasquale Pignatelli. "Platelet oxidative stress and thrombosis." In: Thrombosis research 129.3 (Mar. 2012), pp. 378-381.

[49] David J. J. Waugh and Catherine Wilson. "The interleukin-8 pathway in cancer." In: Clinical Cancer Research 14.21 (Nov. 2008), pp. 6735-6741.

[50] Nan Yang, David W Ray, and Laura C Matthews. "Current concepts in glucocorticoid resistance." In: Steroids 77.11 (Sept. 2012), pp. 1041-1049. 



\title{
LDL AS DRUG CARRIER
}

\section{Gold Nanocrystal Labeling Allows Low-Density Lipoprotein Imaging from the Subcellular to Macroscopic Level ${ }^{1}$}

Iris E. Allijn, Wei Leong, Jun Tang, Anita Gianella, Aneta J. Mieszawska, Francois Fay, Ge Ma, Stewart Russell, Catherine B. Callo, Ronald E. Gordon, Emine Korkmaz, Jan Andries Post, Yiming Zhao, Hans C. Gerritsen, Axel Thran, Roland Proksa, Heiner Daerr, Gert Storm, Valentin Fuster, Edward A. Fisher, Zahi A. Fayad, Willem J. M. Mulder, and David P. Cormode.

\begin{abstract}
Low-density lipoprotein (LDL) plays a critical role in cholesterol transport and is closely linked to the progression of several diseases. This motivates the development of methods to study LDL behavior from the microscopic to whole-body level. We have developed an approach to efficiently load LDL with a range of diagnostically active nanocrystals or hydrophobic agents. We performed focused experiments on LDL labeled with gold nanocrystals (Au-LDL). The labeling procedure had minimal effect on LDL size, morphology, or composition. Biological function was found to be maintained from both in vitro and in vivo experiments. Tumor-bearing mice were injected intravenously with LDL, DiR-LDL, Au-LDL, or a gold-loaded nanoemulsion. LDL accumulation in the tumors was detected with whole-body imaging methods, such as computed tomography (CT), spectral CT, and fluorescence imaging. Cellular localization was studied with transmission electron microscopy and fluorescence techniques. This LDL labeling procedure should permit the study of lipoprotein biointeractions in unprecedented detail.
\end{abstract}

1 Published in ACS Nano, 2013, 7(11), pp 9761-9770, DOI: 10.1021/nn403258w 


\subsection{INTRODUCTION}

Low-density lipoprotein (LDL) plays an important role in the transport of cholesterol to peripheral tissues, [6] which is crucial for the maintenance of cell membranes. [3] LDL consists of a hydrophobic core containing cholesteryl esters, which is covered by a phospholipid monolayer and is $20-25 \mathrm{~nm}$ in diameter. [6, 41, 12] One apolipoprotein (ApoB10o) per LDL nanoparticle is embedded into the phospholipid monolayer, covering about half of its surface. ApoBioo is insoluble, nonexchangeable, and recognized by the LDL receptor (LDLr). [41] LDL is a key contributor to a variety of pathological processes. The accumulation of oxidized LDL in the arterial wall and the consequent induction of inflammation is a main factor in the formation of early atherosclerotic lesions, which can progress into vulnerable plaques that may rupture and cause detrimental events such as myocardial infarctions or strokes. $[1,27,36]$ In addition to cardiovascular diseases, LDL also has a role in the progression of certain tumors as a result of the high demand for cholesterol in tumorigenesis. [12] These important roles of LDL motivate its study and imaging of its biointeractions, in particular.

To facilitate imaging, the core of LDL can be substituted with hydrophobic small molecules by Krieger's method, [26] which allows the inclusion of fluorophores, [48] small molecules,[44] and triglycerides. [19] Despite their high diagnostic and therapeutic potential, [34] to the best of our knowledge, robust methods to load nanocrystals in the core of LDL have not been reported. This is due to the difficulty of reconstituting LDL, as ApoBioo is insoluble and nonexchangeable. [41]

Gold nanoparticles have been used as therapeutics [17] to enhance radiotherapy, for targeted drug delivery, [28] and for photothermal ablation. [31] Gold nanoparticles have also a major role in imaging and are used as contrast agents in subcellular transmission electron microscopy (TEM), [35, 2] fluorescence imaging, [9] photoacoustics, [25] surfaceenhanced Raman spectroscopy (SERS), [21, 24] computed tomography (CT), [16, 40] and most recently, spectral CT, a novel multicolor CT technique. [7] Hence the ability to load LDL with gold nanoparticles would allow LDL to be tracked using various imaging modalities or act as a platform to deliver therapeutically active gold nanocrystals to diseased tissue.

In this study, we report a novel method to label human LDL with a variety of diagnostically active nanocrystals as well as amphiphilic or hydrophobic fluorophores (Figure 4.1). The method relies on encapsulation of the aforementioned compounds in micelles and their subsequent translocation into the LDL core using sonication and ultracentrifugation procedures. We studied gold-loaded LDL (Au-LDL) 
in-depth, determining from analytical, in vitro, and in vivo experiments that its properties are similar to that of native LDL. We will show how these gold-labeled LDL nanoparticles can be tracked and exploited for the visualization of lipoprotein biointeractions in vitro and in a tumor mouse model.

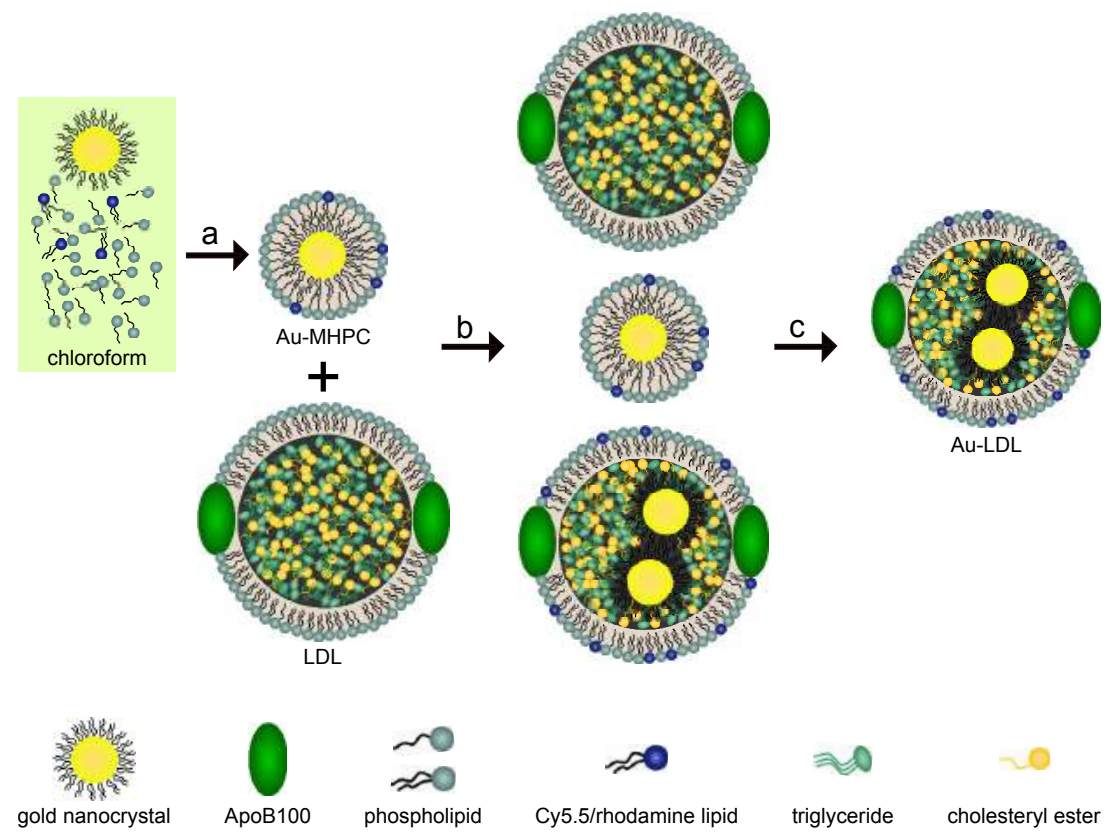

Figure 4.1: Labeling schematic of low-density lipoprotein. Dodecanethiolcoated gold nanocrystals were mixed with phospholipids and Cy5.5 lipids in $\mathrm{CHCl}_{3}$. (a) This solution was dripped into $>70$ ${ }^{\circ} \mathrm{C} \mathrm{H}_{2} \mathrm{O}$, forming micelles enclosing a gold nanocrystal. (b) Phospholipid-coated gold nanocrystals were mixed with native LDL in PBS and sonicated, yielding three different particles: free phospholipid-coated gold nanocrystals, native LDL, and native LDL with gold nanocrystals in the hydrophobic core. (c) This solution was purified on a dual-density gradient, resulting in a solution of gold-labeled LDL nanoparticles (Au-LDL).

\subsection{RESULTS AND DISCUSSION}

\subsubsection{Labeling of Low-Density Lipoprotein}

A novel and simple strategy was used to incorporate gold nanocrystals in the lipid core of LDL. To that end, LDL was isolated from human blood plasma via standard centrifugation procedures. [18] Do- 
Please see chapter 5 curcumin-loaded

LDL. decanethiol coated 2 - $3 \mathrm{~nm}$ gold nanoparticles were synthesized by the method of Brust, [4] subsequently coated with phospholipids, and added to the native LDL solution (Figure 4.2a,b). Sonication of this solution resulted in labeling of LDL with gold cores (Figure 4.2c). A density gradient centrifugation method was optimized to purify the sample and remove unincorporated gold (Figure 4.1). The final product contained LDL of which $77 \%$ was labeled with gold (with an average of a $1.5 \mathrm{Au} / \mathrm{LDL}$ ), as shown in Figure 4.2d. The incorporation of Cy5.5 or rhodamine-labeled phospholipids into LDL can be achieved by their inclusion in the initial phospholipid coating of the gold nanocrystals.

This new labeling method was compared with the method of Krieger, [26] which has been used to substitute the core of LDL with hydrophobic small molecules, such as photosensitizers. [48] We found the sonication method for labeling LDL with gold nanocores to be markedly more efficient than the Krieger method and better preserved LDL's morphology (Figure 4.2e). Gold containing nanoemulsions (Au-NE) (Figure 4.2f) were synthesized using a method we described previously [23] and used as control particles with a similar morphology and diameter as $\mathrm{Au}-\mathrm{LDL}$, but without apolipoprotein ApoBioo.

To investigate the broader applicability of this labeling method, we performed test experiments with iron oxide nanocores $(10 \mathrm{~nm})$, quantum dots (7.5 $\mathrm{nm}$, Supporting Information Figure $\mathrm{S}_{1}$ ), and the hydrophobic fluorophores BODIPY and DIR, of which the latter two acted as model drugs. Each of these compounds was encapsulated in phospholipid micelles and sonicated with LDL to form IO-LDL, QDLDL, BODIPY-LDL, and DiR-LDL, respectively. BODIPY-LDL and DiRLDL were repurified via Havel's centrifugation method [18] to isolate them from any unincorporated label. TEM of these formulations (Figure $4.2 \mathrm{~g}-\mathrm{i}$ ) indicated that the general morphology of LDL was maintained. LDL was found to be labeled with both iron oxides and quantum dots; however, in the case of iron oxides, the nanocores were not homogenously merged into the LDL core. This difference in labeling is likely related to the differing ligands of the iron oxide (oleic acid) as compared to the gold nanocrystals and quantum dots (dodecanethiol), although potentially it could be due to the larger size of the iron oxides.

\subsubsection{Characterization of Labeled LDL}

Transmission electron microscopy (TEM) showed that Au-LDL has the same morphology and size as native human LDL (Figure $4.2 \mathrm{~b}-\mathrm{d}$ and Figure 4.3a), indicating little effect of sonication on these parameters. Au-LDL typically was loaded with $8.3 \mathrm{mg} \mathrm{Au} / \mathrm{mg}$ ApoB1oo. LDL can be oxidized, which alters its selectivity [29] due to chemical changes in ApoB10o. [30] Importantly, an ELISA assay showed no significant 

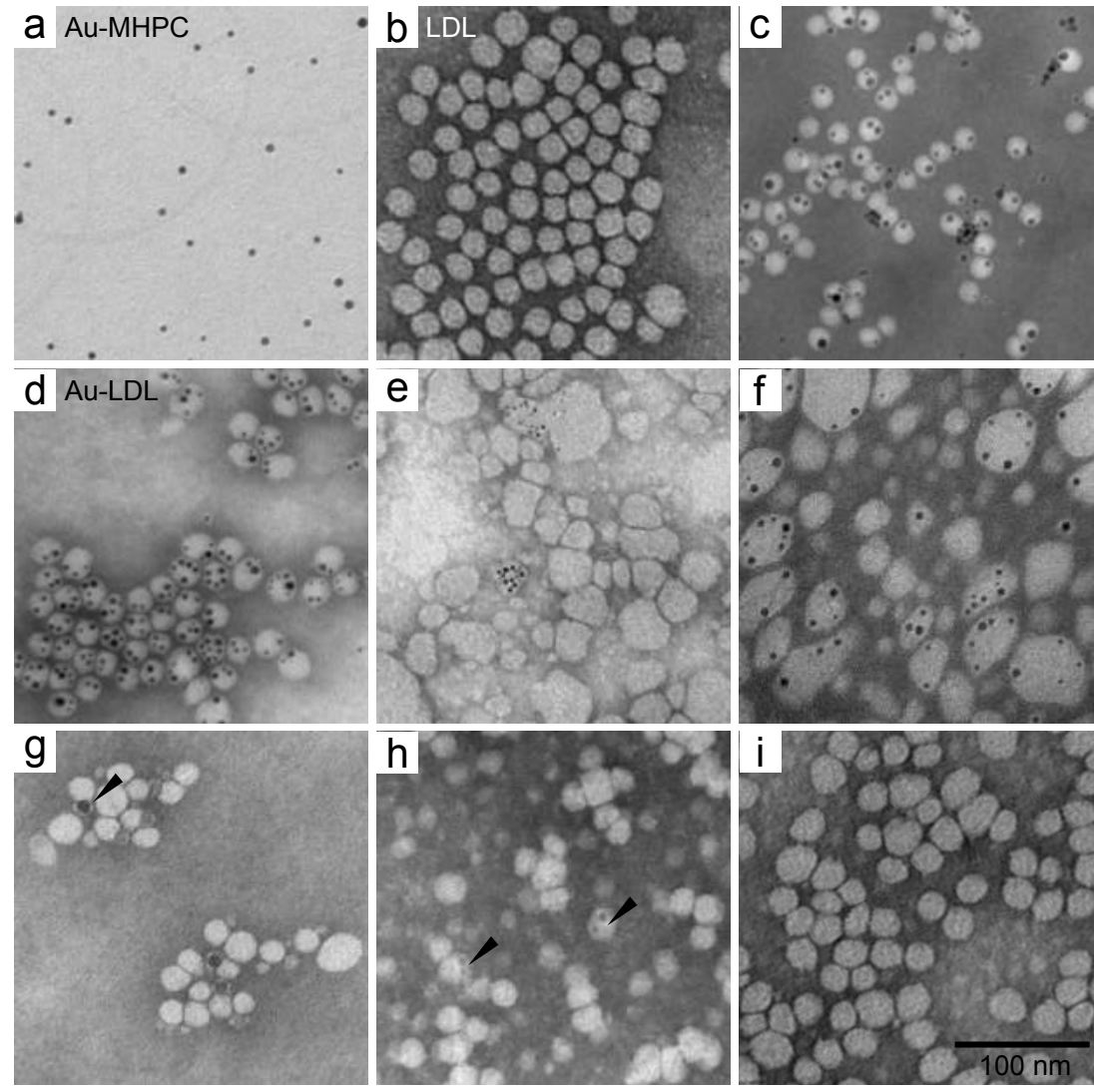

Figure 4.2: LDL labeled with different payloads. Negative stain TEM micrographs of (a) phospholipid-coated gold nanocrystals; (b) native human LDL; (c) unpurified Au-LDL; (d) purified Au-LDL; (e) TEM of LDL labeled with $3 \mathrm{~nm}$ Au nanocrystals using Krieger's reconstitution method.[26] Most LDL nanoparticles stay unlabeled, and their morphology has changed. (f) TEM of Au-NE nanoemulsions, the control particle used in the animal experiments. (g) TEM of 10 $\mathrm{nm}$ IO-LDL. Most LDL stays unlabeled, some cores seem to attach to the side of the LDL, but not enter the core (arrowhead). (h) TEM of QD-LDL. QDs are visible in the core of the LDL (arrowheads). (i) TEM of BODIPY-LDL. Scale bar is the same for all TEM micrographs. $\mathrm{NE}=$ nanoemulsion, $\mathrm{IO}=$ iron oxide, $\mathrm{QD}=$ quantum dot. 
difference in oxidation between LDL and Au-LDL (Figure 4.3b), indicating that the sonication procedure did not affect the oxidation level. LDL had $3.55 \mathrm{mg}$ protein/mM phosphate, while Au-LDL had 2.85 $\mathrm{mg}$ protein $/ \mathrm{mM}$ phosphate as determined by analytical methods. This change is likely due to inclusion of the phospholipids used to coat the gold cores in Au-LDL. Western blots for ApoBioo on LDL and Au-LDL (Figure 4.3C) showed the same molecular weight of ApoBıoo, again indicating no change from sonication. All together, these data corroborated that our labeling technique does not affect the physiochemical integrity of the LDL nanoparticle. From phantoms of Au-LDL imaged with CT, we found the attenuation to be linear in the 0 to $200 \mathrm{mM}$ concentration range, with an attenuation rate of $4.3 \mathrm{HU} / \mathrm{mM} \mathrm{Au}$ at $120 \mathrm{kV}$ (Figure 4.3d,e).

QD-LDL and BODIPY-LDL exhibited strong fluorescence under UV irradiation, while Au-Cy5.5-LDL and DiR-LDL were strongly fluorescent when imaged with a fluorescence imaging system, confirming successful labeling and that the process does not deactivate the fluorophores (Figure $4.3 \mathrm{f}-\mathrm{h}$ ). Due to the hydrophobic nature of the BODIPY and DiR dye molecules, we expect them to be loaded in the core. We chose to perform in-depth in vitro and in vivo experiments with AuLDL, due to its potential for detection with high-resolution techniques such as electron microscopy and computed tomography.

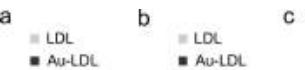
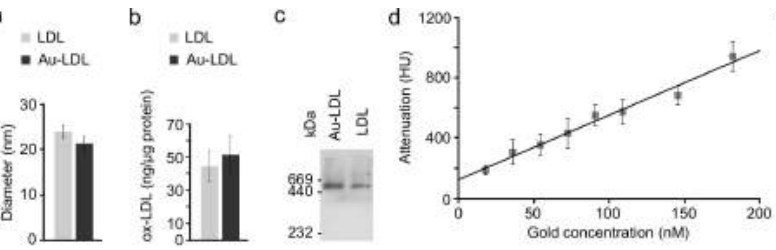

e AU-LOL
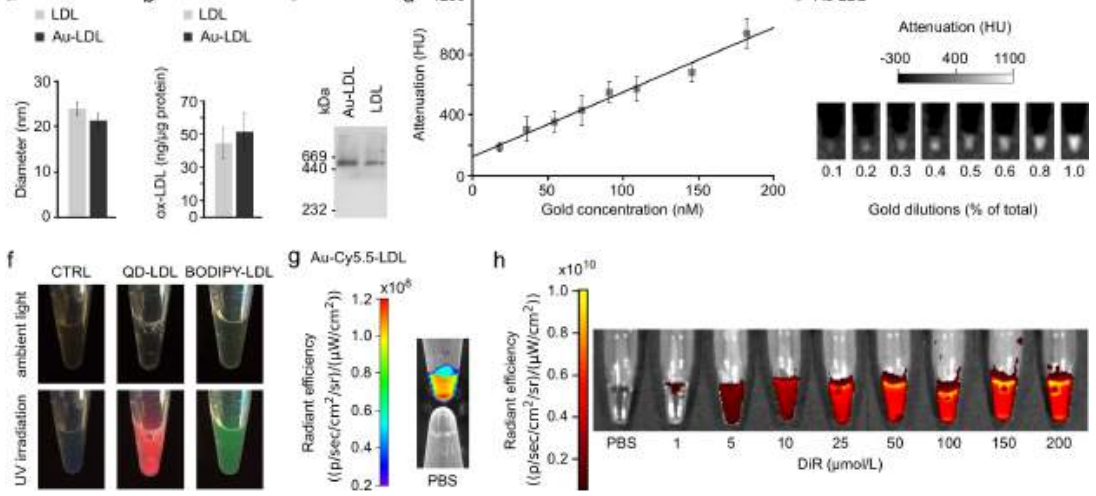

Gold dilutions (\% of total)

Figure 4.3: Characterization of labeled LDL. (a) Diameters of LDL and AuLDL derived from TEM. (b) ELISA for oxidation of LDL and AuLDL. (c) Western blot of ApoBioo of Au-LDL and LDL. (d) Gold concentration-attenuation curve of Au-LDL phantom. (e) Phantom of increasing Au-LDL concentrations. (f) Photograph of CTRL, QDLDL, and BODIPY-LDL in ambient light and under UV irradiation. (g) Phantom of Au-Cy5.5-LDL compared with control PBS. (h) Fluorescence images of DiR-LDL. 


\subsubsection{Labeled LDL Is Taken up in a Receptor-Dependent Fashion in Vitro}

In vitro competition inhibition assays were undertaken in three different cell types known to express the LDLr, that is, hepatocytes (HepG2), [14] macrophages (J774A.1), [38] and melanoma cells (B16-F10). [14] LDLr upregulation was achieved by incubation in DMEM containing 1\% BSA and confirmed by Western blotting (Figure S2). Fluorescence microscopy of these cells incubated with rhodamine-labeled Au-LDL revealed strong nanoparticle uptake, which was reduced when incubations were performed with an excess of LDL (Figure 4.4a-i). The uptake of Au-LDL could also be measured by CT imaging of pellets of the cells (Figure 4.4j-1). Furthermore, TEM showed gold uptake in cells incubated with $\mathrm{Au}-\mathrm{LDL}$ only and reduced uptake in cells incubated with Au-LDL in competition with an excess of native LDL (Figure S3ai). This reduced uptake of Au-LDL when incubated with native LDL in the competition inhibition assay indicates that the nanoparticles are taken up in a receptor-dependent manner, revealing that Au-LDL has preserved biological functionality.

\subsubsection{Labeled LDL Retains Its Function in Vivo}

In order to ensure the in vivo biological functionality of Au-LDL, we performed experiments in wild-type mice and LDLr knockout (KO) mice. LDL has been established to have an extended blood circulation half-life in LDLr KO compared with wild-type mice, as a result of lack of uptake by the LDL receptor. [22] We injected Au-LDL into these mice ( $n=5$ /group), performed blood draws over $24 h$, and measured the gold content of the blood with ICP-MS (Figure 4.5a). We found the half-life of Au-LDL to be $2 \mathrm{~h}$ in wild-type mice and $4 \mathrm{~h}$ in LDLr KO mice, closely matching literature figures of 2 and $5 \mathrm{~h}$ in these animals. [22] Next, we examined the biodistribution of Au-LDL in these mice. We found there to be $50 \%$ higher liver uptake in the wild-type mice as compared to the LDLr KO mice, supporting our findings from pharmacokinetics data (Figure 4.5b). On a cellular level, the presence of the LDL receptor in wild-type mice should lead to substantial uptake in hepatocytes, the main cell type in the liver, whereas the lack of this receptor in LDLr KO mice should result in low hepatocyte uptake and more uptake in Kupffer cells, a minority cell type in the liver. We investigated the liver distribution of Au-LDL using light microscopy of silver stained sections (silver staining makes accumulations of gold nanoparticles visible in light microscopy) and TEM. In light microscopy, we observed darker, diffuse staining of the liver in wild-type mice, whereas we observed punctuate staining in LDLr KO 


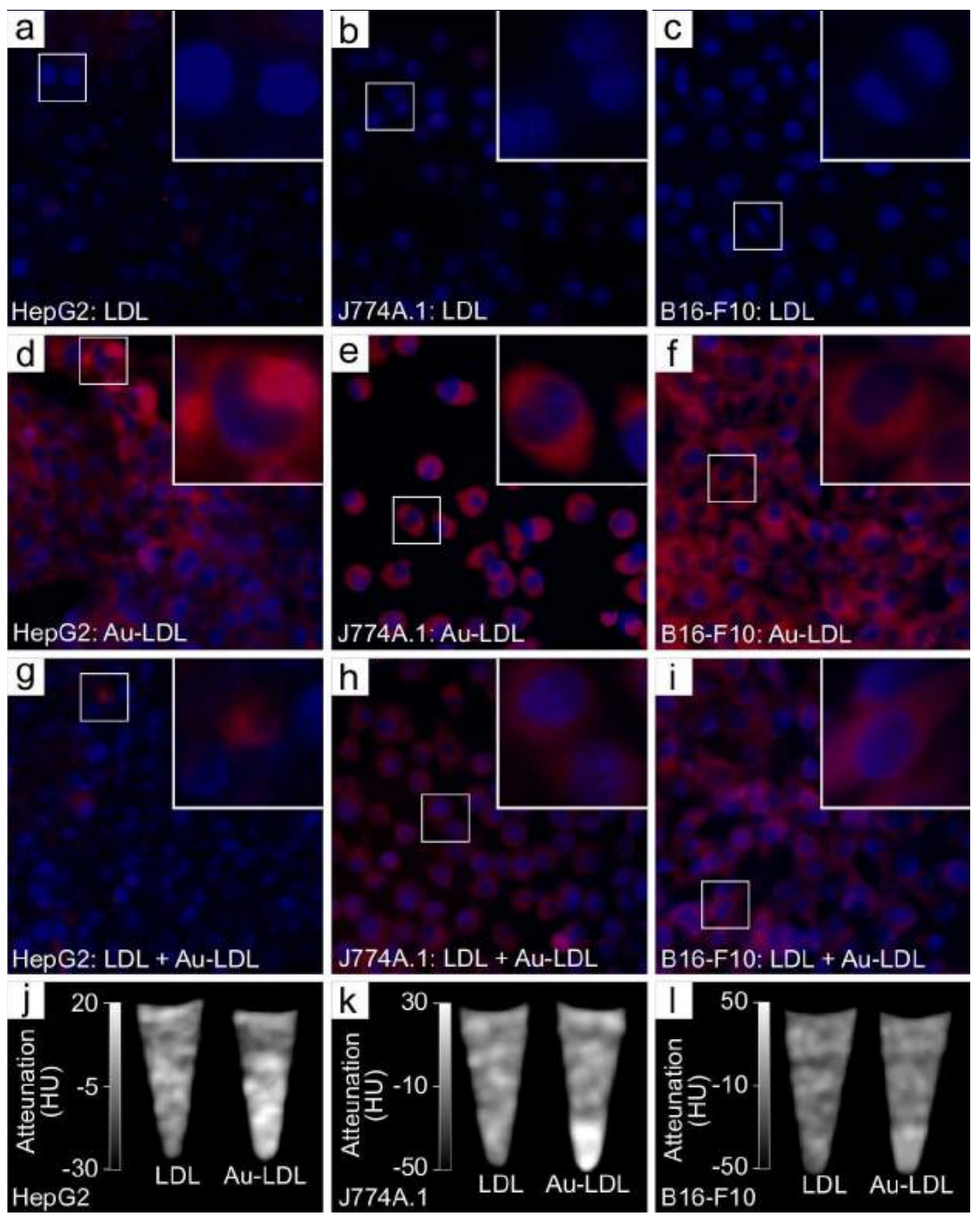

Figure 4.4: In vitro competition inhibition assay. (a) Fluorescence microscopy of LDL (control) (a-c), Au-LDL (test) (d-f), and LDL + Au-LDL (inhibition competition) (g-i) in HepG2, J774A.1 and B16-F1o cells. CT of control and Au-LDL in HepG2 (j), J774A.1 (k), and B16-F1o (l) cells. Red fluorescence is Au-LDL, blue fluorescence is DAPI. 
mice (Figure 4.5c,d). In TEM micrographs, gold cores were found in more vesicles and in greater quantities in hepatocytes in the liver tissue of wild-type mice than LDLr KO mice (Figure 4.5e-h). Thus the cellular distribution of Au-LDL in the liver is consistent with that expected for native LDL. These experiments demonstrate the functionality of Au-LDL in vivo.

\subsubsection{Labeling Allows Macroscopic Imaging of LDL}

Mice bearing tumors that overexpress the LDL receptor were used for in vivo imaging studies (Lewis lung carcinoma or B16-F10). [14, 47] When the tumors reached an average volume of $4 \mathrm{~mm}^{3}$, mice bearing Lewis lung carcinoma tumors were injected intravenously with DiRLDL (DiR is a high quantum yield, near-infrared fluorophore, suited for in vivo imaging). Twenty-four hours after injection, fluorescence imaging of the mice (Figure 4.6a) revealed distinct accumulation in the tumors and, when the mice were dissected, in the liver and spleen as well (Figure $\mathrm{S}_{4}$ ).

Mice bearing B16-F10 tumors were injected with either Au-LDL or $\mathrm{Au}-\mathrm{NE}$ and imaged in vivo with $\mathrm{CT}$ and, after sacrifice, with spectral
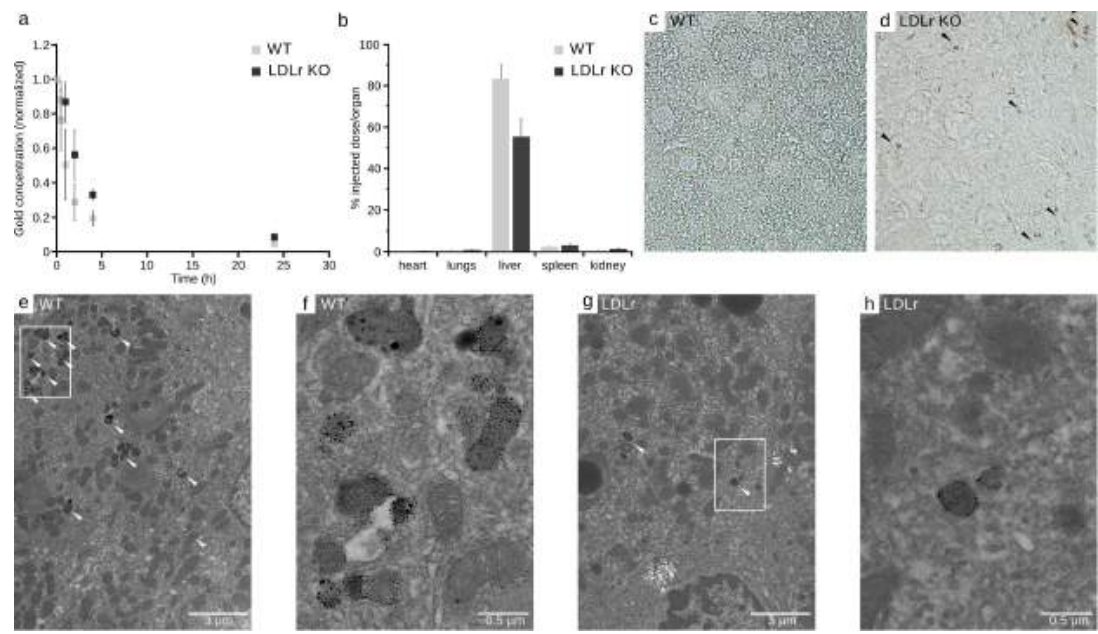

Figure 4.5: In vivo functionality of Au-LDL in wild type and LDLr KO mice.

(a) Pharmacokinetics of Au-LDL in mice. (b) Biodistribution of AuLDL in mice at $2 \mathrm{~h}$ postinjection. (c,d) Light microscopy of silver stained sections of mouse livers. Black arrowheads indicate intense areas of silver staining. (e-h) TEM of hepatocytes in mouse livers. White arrowheads indicate accumulations of gold nanoparticles. $(\mathrm{f}, \mathrm{h})$ Higher magnification images of the boxed areas in panels $\mathrm{e}$ and $g$, respectively. 

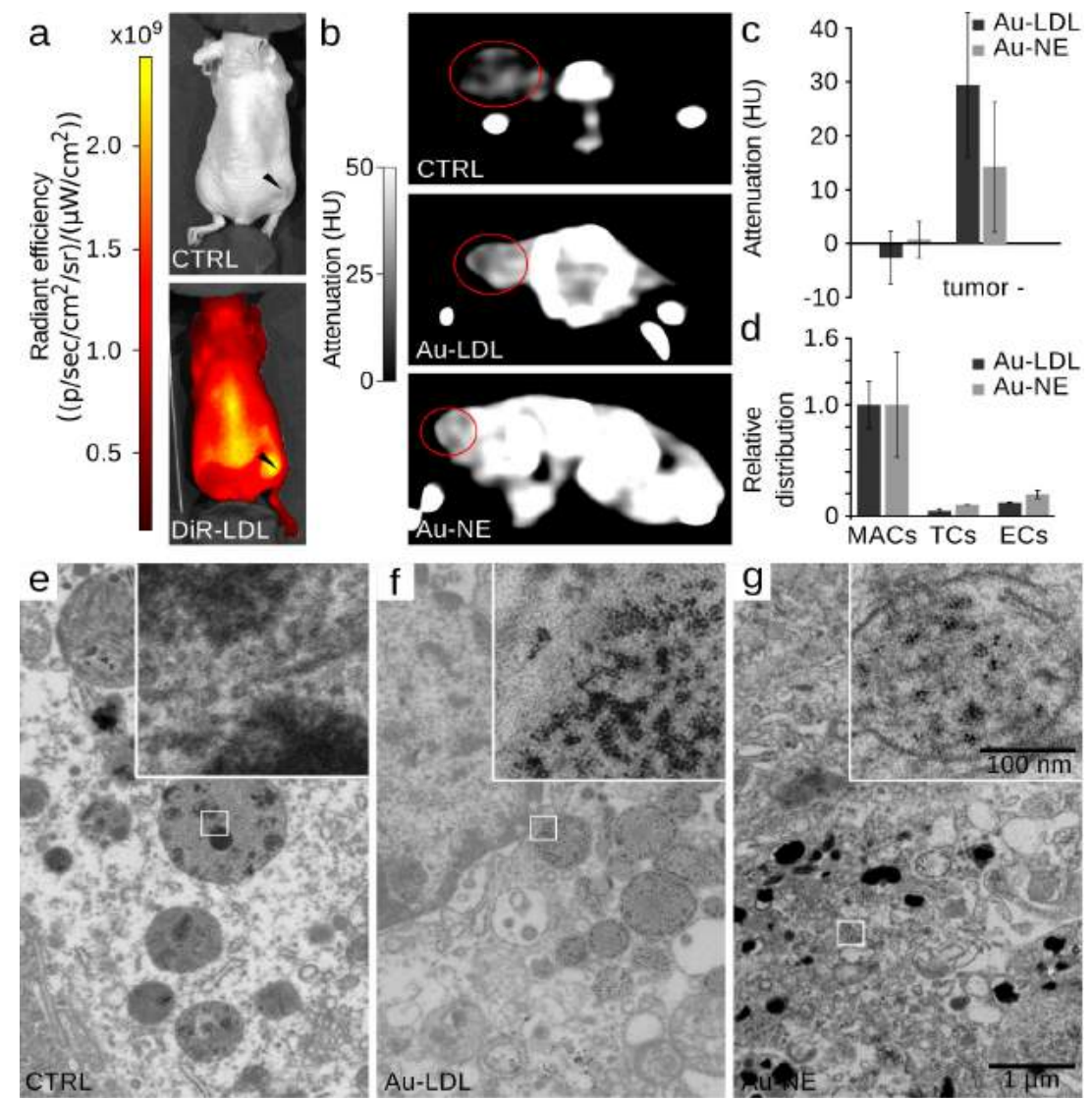

Figure 4.6: In vivo and ex vivo imaging. (a) IVIS of mice; arrowheads indicate the B16-Fio tumor. (b) CT of mice; the tumors are circled. (c) Tumor attenuation relative to control. Attenuation in the tumors of the Au-LDL mice were higher than in the Au-NE mice $(\mathrm{p}=0.009)$. (d) FACS of tumor tissue. MACs, macrophages; TCs, tumor cells; ECs, endothelial cells. (e) TEM of control tumor tissue; inset shows no gold. (f) TEM of Au-LDL tumor tissue; inset shows gold particles in a vesicle. (g) TEM of Au-NE tumor tissue; inset shows gold particles in a vesicle. 
CT (Figures 4.6b,c, 4.7, and $\mathrm{S}_{5}$ ). We observed the attenuation in the tumors to be higher for Au-LDL than for Au-NE injected mice ( $p=$ 0.009 , Figure 4.6c). Spectral CT showed clear accumulation of gold at the rim of the tumor (Figures 4.7a and $\mathrm{S}_{5} \mathrm{a}$ ) and accumulation in the liver (Figures $4.7 \mathrm{~b}$ and $\mathrm{S}_{5} \mathrm{~b}$ ); which is as expected considering the LDLr expression in these tissues. Significant accumulations of gold in other tissues were not observed.

\subsubsection{Cellular and Subcellular Localization of LDL}

FACS analysis of the tumors revealed that the majority of both AuLDL and Au-NE was taken up by macrophages, while smaller fractions of the nanoparticles were found in endothelial cells and cancer cells (Figure 4.6d). In TEM micrographs of tumor tissue of control mice, tumor cells with melanosomes, tumor-associated macrophages (TAMs), muscle cells, and endothelial cells could be identified. In tumor tissue of mice injected with Au-LDL or Au-NE, highly electron scattering regions were observed inside lysosomal structures in tumor cells, macrophages, and endothelial cells. Such highly electron scattering regions were absent in control tumor tissue, and EDX element analysis confirmed that these were gold (Figure S6). Visual inspection indicated that more gold nanospheres were observed in Au-LDL tumor
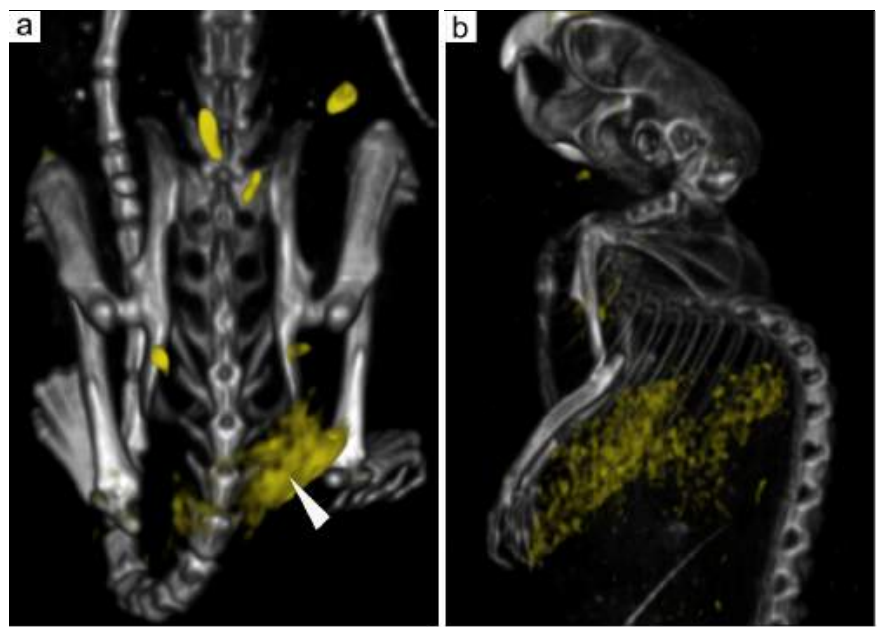

Figure 4.7: Spectral CT of Au-LDL injected B16-Fio tumor-bearing mice. Gold accumulation is displayed in yellow and overlaid on conventional CT images. (a) Three-dimensional reconstruction of the rear of a mouse injected with Au-LDL. Gold accumulation can be observed in the tumor. (b) Three-dimensional reconstruction of the front of a mouse with gold accumulation found in the liver. 
tissue than in Au-NE tumor tissue. Three-dimensional reconstructions from electron tomography of tissue showed that tumor vesicles were extensively loaded with gold nanoparticles (Figure $\mathrm{S}_{7}$ ).

We established a new LDL labeling method based on sonication followed by ultracentrifugation purification. Using this method, we were able to label native human LDL with a variety of payloads, and in this study, we reported the first gold-labeled LDL (Au-LDL) for CT, fluorescence techniques, and TEM. This sonication-based loading method, compared to the Krieger method, [26] proved to be far more effective in terms of labeling efficiency and morphology conservation (Figure 4.2a-e). We have shown Au-LDL to have similar characteristics to native LDL in terms of size, morphology, composition, oxidation state, ApoBioo function, and molecular weight. Au-LDL was taken up in a receptor-like, saturable manner and could be detected by fluorescencebased techniques, TEM, and CT in a variety of LDLr-expressing cell types. Experiments performed in LDLr KO and wild-type mice confirmed LDL-like behavior in vivo. In comparison to the currently available labeling methods, our method has the advantage that it can be applied to a variety of nanocrystals, hydrophobic molecules, and, we expect, lipoproteins, which makes it of broad relevance.

In the current study, the in vivo application in B16-F1o tumor-bearing mice revealed that the gold nanoparticles were mostly associated with TAMs. The uptake of Au-LDL in the TAMs is likely due to both LDLrmediated uptake and nonspecific uptake via phagocytosis after the Au-LDL has accumulated in the tumor via the enhanced permeability and retention effect. Recent studies have shown TAMs to be an interesting therapeutic target, as their number is inversely correlated with patient survival. [45] For example, Edris et al. showed that treatment with antibodies against $\mathrm{CD}_{47}$, a factor signaling between tumor cells and macrophages, resulted in a decrease in the size and the number of metastases. [10] To be able to detect TAMs by CT imaging may allow monitoring of early responses to anti-TAM therapy in a quantitative fashion and may also allow predictions of therapeutic outcome.

We have clearly demonstrated that labeling LDL with gold cores enables detection with CT, TEM, and fluorescence-based techniques, permitting LDL localization from the whole-body level to the subcellular level. Others have previously labeled LDL with radionuclides for imaging with nuclear-based methods. [15, 43, 42] In addition, LDL has been labeled with fluorophores for fluorescence imaging and gadolinium chelates for MRI. [6, 14, 5, 49] Such imaging methods are more sensitive than CT, but nuclear imaging has poor resolution and cellular localization of injected LDL is difficult with the labels used for either nuclear imaging or MRI. The use of spectral CT, as we showed here, allows detection of Au-LDL accumulation in a "hot-spot" fashion, without the need for comparison of pre- and postinjection images as 
for MRI. This work is notable also, as Au-LDL represents one of only a few reports of targeted CT contrast agents. Potentially, Au-LDL could be "retargeted" to other receptors or cell types by attaching additional targeting ligands to the ApoBioo component, as has been reported by Zheng et al.. [49]

LDL has been proposed as a drug delivery vehicle. For example, Firestone et al. loaded LDL with cytotoxic cholesterol oleate appended nitrogen mustards and observed specific uptake of the nanoparticles as well as complete cell death in vitro. [11] The method used in the current study (i.e., cosonication of LDL with gold nanocores coated with phospholipids) could also be applied to load LDL with hydrophobic drugs, as our experiments with BODIPY and DiR indicate. As we have shown, iron oxides, quantum dots, hydrophobic fluorophores, or drugs could also be included to create a multifunctional or "theranostic" platform. [46]

\section{$4 \cdot 3$ CONCLUSIONS}

In this study, we established a novel sonication method for core labeling native LDL particles with $2-3 \mathrm{~nm}$ gold nanocrystals, which is far more efficient than the well-established Krieger method. Furthermore, this sonication-based labeling method of LDL could be applied to incorporate iron oxide nanocores, QDs, and hydrophobic drugs, allowing LDL to be studied with MRI and optical imaging as well as be used as a nanosized drug delivery system. Native LDL loaded with gold nanocrystals has a comparable composition and morphology to untreated LDL and, importantly, has no change in oxidation level. Gold labeling permitted detection of LDL on the subcellular (TEM), cellular (fluorescence), and anatomical level (CT and spectral CT). Au-LDL was taken up by macrophages, hepatocytes, and a melanoma cell line in vitro in a receptor-dependent manner as determined by the aforementioned techniques. Au-LDL has different pharmacokinetics, liver uptake, and liver cellular distribution in wild-type compared to LDLr KO mice, indicating that it has LDL-like functionality in vivo. Furthermore, investigations in tumor-bearing mice with Au-LDL enabled study of LDL uptake by tumors by both conventional and spectral CT, in vivo fluorescence imaging, and ex vivo microscopy methods. Therefore, AuLDL can likely be used as a marker to study LDL interactions, cholesterol metabolism, atherosclerotic plaque formation, and tumor growth with the imaging techniques used herein and others such as photoacoustics as well as be used to deliver gold nanomaterials for therapeutic purposes. 


\subsection{MATERIALS AND METHODS}

For detailed materials and methods, please see Supporting Information.

\subsubsection{Synthesis}

\subsubsection{Au-Cy5.5-MHPC Nanoparticle Synthesis}

MHPC-coated gold nanocores were synthesized in a similar method to that described previously by Cormode et al.. [8] Typically, $100 \mathrm{mg}$ of MHPC dissolved in $10 \mathrm{~mL}$ of $20: 1 \mathrm{CHCl}_{3} / \mathrm{CH}_{3} \mathrm{OH}, 100 \mathrm{mg}$ of dodecanethiol coated gold nanocores (synthesized via the Brust method [4] dissolved in $5 \mathrm{~mL}$ of $\mathrm{CHCl}_{3}$, and $1 \mathrm{mg}$ of $\mathrm{Cy} 5.5$-DMPE in $\mathrm{CHCl}_{3}$ were mixed and dripped into $10 \mathrm{~mL}>70^{\circ} \mathrm{C} \mathrm{diH}_{2} \mathrm{O}$ at $60 \mu \mathrm{L} / \mathrm{min}$ speed. The resulting solution was concentrated to $7.2 \mathrm{~mL}$ and purified by centrifuging $200 \mu \mathrm{L}$ on $1 \mathrm{~mL}$ of $1.25 \mathrm{mg} / \mathrm{mL} \mathrm{KBr}$ per vial at 14,500 rpm for $1 \mathrm{~h}$ using an Eppendorf Minispin Plus. Afterward, the top 250 $\mu \mathrm{L}$ was taken off and discarded, the remaining solution was collected, washed once with PBS using a 10,000 MWCO tube, and concentrated to $4.8 \mathrm{~mL}$. The $1.25 \mathrm{~g} / \mathrm{mL}$ gradient spin at $14,500 \mathrm{rpm}$ was repeated, and again the top $250 \mu \mathrm{L}$ was taken off and the remaining solution collected. The solution was washed twice with PBS and concentrated to $500 \mu \mathrm{L}$. The Au concentration was determined using CT imaging, and $\mathrm{Au}-\mathrm{Cy}$ 5.5-MHPC was stored in the dark at $4{ }^{\circ} \mathrm{C}$.

\subsubsection{LDL Labeling with Au-Cy5.5-MPHC}

Native human LDL (1.7 mg, by protein content) in PBS was mixed with Au-Cy5.5-MHPC in PBS, containing $4.74 \mathrm{mg}$ of gold. PBS was added to make a total volume of $2 \mathrm{~mL}$, and the solution was sonicated in a water bath for 5 min. Unlabeled LDL particles and Au-Cy5.5-MHPC aggregates were removed via centrifugation on a dual $(700 \mu \mathrm{L} 1.06 \mathrm{mg} / \mathrm{mL}$ on top of $300 \mu \mathrm{L} 1.35 \mathrm{mg} / \mathrm{mL}$ ) $\mathrm{KBr}$ density gradient. Then, $200 \mu \mathrm{L}$ of the sonicated sample was pipetted on top of the $\mathrm{KBr}$ gradient and spun for $6 \mathrm{~h}$ at 10,000 rpm in an Eppendorf Minispin Plus. Subsequently, the top $\sim 400 \mu \mathrm{L}$ of light pink layer (containing unlabeled LDL and other lipid residues) was removed and discarded, and the next $\sim 700 \mu \mathrm{L}$ was collected, leaving the pellet (containing Au-Cy5.5-MHPC aggregates) undisturbed. Au-Cy5.5-LDL (Au-LDL) was washed twice with PBS using 10,000 MWCO tubes, concentrated to $100 \mu \mathrm{L}$, and stored at $4{ }^{\circ} \mathrm{C}$. This procedure was performed repeatedly, and the batches were combined. 


\subsubsection{LDL Labeling Using Core Reconstitution}

LDL was also labeled using the well-known Krieger method [26] as a comparison. To this end, $500 \mu \mathrm{L}$ of native human LDL in $\mathrm{diH}_{2} \mathrm{O}$ and $25 \mathrm{mg}$ of starch (Sigma-Aldrich), for LDL stability, were frozen with a combination of ethanol and dry ice and subsequently lyophilized. The core of LDL was not extracted; instead $6 \mathrm{mg}$ of Au-MHPC in $200 \mu \mathrm{L}$ heptane was added to enter the core. The mixture was placed in the freezer for $10 \mathrm{~min}$ and dried with $\mathrm{N}_{2}$ gas. Dried samples were kept on ice and resuspended in $1 \mathrm{~mL}$ of $10 \mathrm{mM}$ tricine $(\mathrm{pH}$ 4) and left for $20 \mathrm{~h}$ at $4{ }^{\circ} \mathrm{C}$ to release the LDL from the starch. Samples were then centrifuged at 2,000 rpm for $10 \mathrm{~min}$ in a $5810 \mathrm{R}$ Eppendorf centrifuge followed by $10,000 \mathrm{rpm}$. Success of labeling was measured using negative stain TEM (see Characterization section).

\subsubsection{LDL Labeling with QD-MHPC}

The preparation of the quantum dots (QDs) used has been previously reported. [33] Their optical properties are displayed in Figure S1. Their capping ligands were exchanged by incubation with an excess of dodecanethiol in $\mathrm{CHCl}_{3}$ for $72 \mathrm{~h}$ and subsequent isolation by precipitation with $\mathrm{CH}_{3} \mathrm{CH}_{2} \mathrm{OH}$, centrifugation and repeated washing. Then 2.5 nmol of these QDs was dissolved in $5 \mathrm{~mL}$ of a 9:1 $\mathrm{CHCl}_{3} / \mathrm{CH}_{3} \mathrm{OH}$ solvent mixture that contained $12 \mathrm{mg}$ of MHPC. This solution was dripped into $>70{ }^{\circ} \mathrm{C} \mathrm{diH}{ }_{2} \mathrm{O}$ to form QD-MHPC and purified as per the methods used for Au-MHPC. The volume of the solution was adjusted to 900 , and $225 \mu \mathrm{L}$ was mixed with $0.8 \mathrm{mg}$ of LDL (by protein content). The volume was increased to $1 \mathrm{~mL}$ with PBS, and the solution was sonicated for $5 \mathrm{~min}$.

\subsubsection{LDL Labeling with BODIPY or DiR}

The hydrophobic fluorophores BODIPY (4,4-difluoro-1,3,5,7-tetramethyl-4-bora-3a,4a-diaza-s-indacene (BODIPY 505/515)) and DiR (1,1'diocta-decyl-3,3,3', $3^{\prime}$-tetramethylindotricarbocyanine iodide) were purchased from Invitrogen. In the case of BODIPY, $5 \mathrm{mg}$ was dissolved in $10 \mathrm{~mL}$ of a 9:1 $\mathrm{CHCl}_{3} / \mathrm{CH}_{3} \mathrm{OH}$ solvent mixture that contained 100 $\mathrm{mg}$ of MHPC. This solution was dripped into $>70{ }^{\circ} \mathrm{C} \mathrm{diH}{ }_{2} \mathrm{O}$ to form BODIPY-MHPC. Unincorporated BODIPY fluorophore was removed by centrifugation at 4,000 rpm for 5 min using a 5810R Eppendorf centrifuge. Then, $160 \mu \mathrm{L}$ of BODIPY-MHPC was mixed with $17 \mathrm{mg}$ of LDL (by protein content), and the volume was increased to $20 \mathrm{~mL}$ with PBS. This solution was sonicated for $10 \mathrm{~min}$ and was then purified by repeating the LDL isolation process (see section 4.4.1.2) to exclude any BODIPY unincorporated into LDL. In the case of DiR, the same pro- 
cedure was used except $25 \mathrm{mg}$ of MHPC was used and $338 \mu \mathrm{L}$ of the resulting solution was sonicated with LDL.

\subsubsection{Nanoemulsion Synthesis}

Control nanoemulsions (Au-NE) with a similar size and composition to the LDL nanoparticles, but lacking the ApoBioo lipoprotein, were prepared by first incubating $18 \mathrm{mg}$ of dodecanethiol-coated gold cores in $\mathrm{CHCl}_{3}$ with cis-9-octadene-1-thiol for $10 \mathrm{~min}$ for ligand exchange. Next, $12 \mathrm{mg}$ of DSPE-PEG, $5.6 \mathrm{mg}$ of DSPC, $1.16 \mathrm{mg}$ of cholesterol, $20 \mathrm{mg}$ of soybean oil, and $0.176 \mathrm{mg}$ of $\mathrm{Cy} 5.5$ in $\mathrm{CHCl}_{3}$ were mixed, and the Au was added to this solution. A lipid film was made and subsequently hydrated with $5 \mathrm{~mL}$ of $65{ }^{\circ} \mathrm{C}$ PBS. The solution was tipsonicated for $15 \mathrm{~min}$ and concentrated to $500 \mu \mathrm{L}$ using 10,000 MWCO tubes.

\subsubsection{Characterization}

Nanoparticle size and morphology were determined by negative stain TEM, [13] the ApoB1oo concentration by a modified Lowry assay; [32] the gold concentration was determined by $\mathrm{CT}$, and the phosphorus content with Rouser's [37] phosphorus assay. Oxidation of LDL was tested with ELISA, and Western blotting was performed for ApoBioo.

\subsubsection{In Vitro Experiments}

HepG2 hepatocytes, J774A.1 macrophages, and B16-F10 melanoma cells were cultured as per the supplier's instructions. Prior to Au-LDL uptake experiments, the cells were passaged into 6-well plates and washed with $1 \%$ BSA DMEM medium (no FBS) and incubated with this preincubation medium for $20 \mathrm{~h}$ to upregulate LDLr, [48] as confirmed by Western blotting. Cells were incubated with LDL (control), Au-LDL (test), or Au-LDL and a 5-fold excess of LDL (competition inhibition). The concentrations used for in vitro experiments depended on the sensitivity of the imaging equipment used. For TEM, LDL with a concentration of $100 \mu \mathrm{g}$ of $\mathrm{ApoB} 100 / \mathrm{mL}$ was used, for fluorescence, 20 $\mu \mathrm{g}$ of ApoB1oo/mL, and for CT, $500 \mu \mathrm{g}$ of ApoBioo/mL. Cells were washed and harvested as pellets for either TEM or CT analysis. For fluorescence microscopy, the same was performed with cells grown on glass coverslips and mounted on slides with DAPI-containing mounting medium. 


\subsubsection{Animal Experiments}

All experiments were approved by the Institutional Animal Care and Use Committee. To investigate Au-LDL functionality in vivo, we used wild-type $\mathrm{C}_{57} \mathrm{BL} / 6$ mice and LDLr KO mice from the same background (Jackson Laboratories). For pharmacokinetics, Au-LDL was injected into the mice via the tail vein $(\mathrm{n}=5 /$ group, $12 \mathrm{mg} \mathrm{Au} / \mathrm{kg})$ and blood drawn via retro-orbital bleeds at $5 \mathrm{~min}, 30 \mathrm{~min}, 1 \mathrm{~h}, 2 \mathrm{~h}, 4 \mathrm{~h}$, and 24 h. For biodistribution, Au-LDL was injected into the mice via the tail vein $(\mathrm{n}=5 /$ group, $12 \mathrm{mg} \mathrm{Au} / \mathrm{kg}$ ). The mice were sacrificed, perfused with PBS, and dissected. Small pieces of the livers were retained for microscopy analysis. The gold content of the blood samples and organs was determined via ICP-MS performed by the Pennsylvania Animal Diagnostic Laboratory System, Kennett Square, PA. One millimeter cubes of liver were fixed in $2.5 \%$ glutaraldehyde and prepared for TEM analysis by the normal methods, [20] and $3 \mathrm{~mm}$ cubes of liver were embedded in paraffin, sectioned, and stained using a Silver Enhancer Kit (Sigma Aldrich) according to the manufacturers instructions.

For fluorescence imaging, four 7 week old female NCR/NU mice were injected in the right flank with $100 \mu \mathrm{L}$ of DMEM containing 2 million Lewis lung cancer cells. On day 7, mice were injected with DiRLDL $(n=3)$ or PBS $(n=1)$. Fluorescence in the mice was measured using a Xenogen IVIS Spectrum (Alameda, CA) at $24 \mathrm{~h}$ postinjection. Tissue was excited at $745 \mathrm{~nm}$, and the emission spectra were recorded from $820 \mathrm{~nm}$. The mice were subsequently sacrificed, perfused, and their excised organs imaged under the same conditions.

For CT imaging experiments, eleven 7 week old female NCR/ NU mice were injected in the right flank with $100 \mu \mathrm{L}$ of DMEM containing 1 million B16-Fio cells. On day 7, tumors reached the size of $4 \mathrm{~mm}^{3}$, and the mice were divided into four groups by tumor size and subsequently randomly divided into control $(n=4)$, Au-LDL $(n=4)$, and $\mathrm{Au}-\mathrm{NE}(\mathrm{n}=3)$ groups. The nanoparticles were injected in the tail vein at $250 \mathrm{mg} \mathrm{Au} / \mathrm{kg}$ doses $24 \mathrm{~h}$ before imaging and sacrifice. Mice were scanned by CT $(120 \mathrm{kV}, 256$-slice clinical scanner), imaging the distribution of the nanoparticles in the mice. Of each group, two mice were anesthetized with isoflurane and sacrificed using heart perfusion, and tumors were removed. The remaining mice were sacrificed using $\mathrm{CO}_{2}$, snap-frozen with liquid $\mathrm{N}_{2}$, and subsequently scanned with spectral CT (a custom-built device, Philips Research Europe, Hamburg). [39] This scanner uses photon-counting detectors that analyze the energy of the incident X-rays to identify the materials in the field of view. In this case, it was used to identify the gold distribution in the mice. 


\subsubsection{Ex Vivo Experiments}

Excised tumor tissue was used for FACS and TEM. FACS analysis was performed against tumor cells (SSC anti CD31), endothelial cells (CD $31+C D-11 b-$ Gr-1-), and macro phages (CD11b+ Gr-1+). TEM was used to localize the gold in the cells and study the distribution, EDX to confirm the nature of the gold nanoparticles, and ET to confirm that the gold particles were inside vesicles in the cells.

\section{Conflict of Interest}

The authors declare no competing financial interest.

\section{Acknowledgment}

This work was supported by the National Heart, Lung, and Blood Institute, National Institutes of Health, as a Program of Excellence in Nanotechnology (PEN) Award Contract \#HHSN268201000045C, the NIH Grants Roo EBo12165 (D.P.C.), Ro1 EBoog638 (Z.A.F.), and Ro1 CA155432 (W.J.M.M.), the Dutch Heart Foundation (I.E.A.), the Dutch Society for Microscopy (I.E.A.), and the Master Mobility Scholarship (I.E.A.). Furthermore, we thank S.-H. Chen of Mount Sinai School of Medicine for her input on the FACS analysis, and S. Lund-Katz for the gift of a sample of LDL.

\section{Supporting Information}

Data on the optical properties of the quantum dots used. Western blotting of LDL receptor expression in the cell lines used is shown, as is TEM of Au-LDL uptake in these cells. Fluorescence images of the organs of DiR-LDL injected mice and transverse spectral CT images of $\mathrm{Au}-\mathrm{LDL}$ injected mice are provided. Detailed methods are given for various experiments. This material is available free of charge via the Internet at http:/ / pubs.acs.org.

\section{REFERENCES}

[1] JA Berliner and JW Heinecke. "The role of oxidized lipoproteins in atherogenesis." In: Free Radical Biology and Medicine 20.5 (Jan. 1996), pp. 707-727.

[2] Erik Bos et al. "A new approach to improve the quality of ultrathin cryo-sections; its use for immunogold EM and correlative electron cryo-tomography." In: Journal of structural biology 175.1 (July 2011), pp. 62-72. 
[3] M S Brown and J L Goldstein. "Receptor-mediated endocytosis: insights from the lipoprotein receptor system." In: Proceedings of the National Academy of Sciences of the United States of America 76.7 (July 1979), pp. 3330-7.

[4] Mathias Brust et al. "Synthesis of thiol-derivatised gold nanoparticles in a two-phase Liquid-Liquid system." In: Journal of the Chemical Society, Chemical Communications 7 (1994), p. 801.

[5] Juan Chen et al. "Ligand conjugated low-density lipoprotein nanoparticles for enhanced optical cancer imaging in vivo." In: Journal of the American Chemical Society 129.18 (May 2007), pp. 5798-9.

[6] Ian R Corbin et al. "Low-density lipoprotein nanoparticles as magnetic resonance imaging contrast agents." In: Neoplasia (New York, N.Y.) 8.6 (June 2006), pp. 488-98.

[7] David P Cormode et al. "Atherosclerotic plaque composition: analysis with multicolor CT and targeted gold nanoparticles." In: Radiology 256.3 (Sept. 2010), pp. 774-782.

[8] David P Cormode et al. "Nanocrystal core high-density lipoproteins: a multimodality contrast agent platform." In: Nano letters 8.11 (Nov. 2008), pp. 3715-23.

[9] Nicholas J Durr et al. "Two-photon luminescence imaging of cancer cells using molecularly targeted gold nanorods." In: Nano letters 7.4 (Apr. 2007), pp. 941-5.

[10] Badreddin Edris et al. "Antibody therapy targeting the $\mathrm{CD}_{47}$ protein is effective in a model of aggressive metastatic leiomyosarcoma." In: Proceedings of the National Academy of Sciences 109.17 (Apr. 2012), pp. 6656-6661.

[11] R A Firestone et al. "Selective delivery of cytotoxic compounds to cells by the LDL pathway." In: Journal of medicinal chemistry 27.8 (Aug. 1984), pp. 1037-1043.

[12] Raymond A. Firestone. "Low-Density Lipoprotein as a Vehicle for Targeting Antitumor Compounds to Cancer Cells." In: Bioconjugate Chemistry 5.2 (Mar. 1994), pp. 105-113.

[13] TM Forte and RW Nordhausen. "Electron microscopy of negatively stained lipoproteins." In: Methods in enzymology 128.Part 2 (Jan. 1986), pp. 442-57.

[14] Simonetta Geninatti Crich et al. "Magnetic Resonance Imaging Detection of Tumor Cells by Targeting Low-Density Lipoprotein Receptors with Gd-Loaded Low-Density Lipoprotein Particles." In: Neoplasia 9.12 (Dec. 2007), pp. 1046-1056. 
[15] H N Ginsberg, S J Goldsmith, and S Vallabhajosula. "Noninvasive imaging of 99mtechnetium-labeled low density lipoprotein uptake by tendon xanthomas in hypercholesterolemic patients." In: Arteriosclerosis (Dallas, Tex.) 10.2 (1990), pp. 256-62.

[16] J F Hainfeld et al. "Gold nanoparticles: a new X-ray contrast agent." In: The British journal of radiology 79.939 (Mar. 2006), pp. 248-53.

[17] James F Hainfeld, Daniel N Slatkin, and Henry M Smilowitz. "The use of gold nanoparticles to enhance radiotherapy in mice." In: Physics in Medicine and Biology 49.18 (Sept. 2004), N309-N315.

[18] RJ Havel, HA Eder, and JH Bragdon. "The distribution and chemical composition of ultracentrifugally separated lipoproteins in human serum." In: Journal of Clinical Investigation 34.9 (Sept. 1955), pp. 1345-53.

[19] Melissa L Hill et al. "In vitro assessment of poly-iodinated triglyceride reconstituted low-density lipoprotein: initial steps toward CT molecular imaging." In: Academic radiology 17.11 (Nov. 2010), pp. 1359-65.

[20] Daniel Horak et al. "D-mannose-modified iron oxide nanoparticles for stem cell labeling." In: Bioconjugate chemistry 18.3 (2007), pp. 635-44.

[21] Xiaohua Huang et al. "Cancer cells assemble and align gold nanorods conjugated to antibodies to produce highly enhanced, sharp, and polarized surface Raman spectra: a potential cancer diagnostic marker." In: Nano letters 7.6 (June 2007), pp. 1591-7.

[22] S Ishibashi et al. "Hypercholesterolemia in low density lipoprotein receptor knockout mice and its reversal by adenovirus-mediated gene delivery." In: The Journal of clinical investigation 92.2 (Aug. 1993), pp. 883-93.

[23] P. A. Jarzyna, Torjus Skajaa, and Anita Gianella. "Iron oxide core oil-in-water emulsions as a multifunctional nanoparticle platform for tumor targeting and imaging." In: Biomaterials 30.36 (Dec. 2009), pp. 6947-54.

[24] S Keren et al. "Noninvasive molecular imaging of small living subjects using Raman spectroscopy." In: Proceedings of the National Academy of Sciences of the United States of America 105.15 (Apr. 2008), pp. 5844-9.

[25] Moritz F. Kircher et al. "A brain tumor molecular imaging strategy using a new triple-modality MRI-photoacoustic-Raman nanoparticle." In: Nature medicine 18.5 (May 2012), pp. 829-34. 
[26] Monty Krieger. "Reconstitution of the hydrophobic core of low-density lipoprotein." In: Methods in enzymology 128.1977 (Jan. 1986), pp. 6o8-13.

[27] P Libby. "Cholesterol and atherosclerosis." In: Biochim. Biophys. Acta - Mol. Cell Biol. Lipids 1529.1-3 (Dec. 2000), pp. 299-309.

[28] Steven K Libutti et al. "Phase I and pharmacokinetic studies of CYT-6091, a novel PEGylated colloidal gold-rhTNF nanomedicine." In: Clinical cancer research : an official journal of the American Association for Cancer Research 16.24 (Dec. 2010), pp. 6139-49.

[29] M Lougheed and U P Steinbrecher. "Mechanism of uptake of copper-oxidized low density lipoprotein in macrophages is dependent on its extent of oxidation." In: The Journal of biological chemistry 271.20 (May 1996), pp. 11798-805.

[30] S Lund-Katz et al. "Apolipoprotein B-10o conformation and particle surface charge in human LDL subspecies: implication for LDL receptor interaction." In: Biochemistry 37.37 (Sept. 1998), pp. 12867-74.

[31] Geoffrey von Maltzahn et al. "Computationally guided photothermal tumor therapy using long-circulating gold nanorod antennas." In: Cancer research 69.9 (May 2009), pp. 3892-900.

[32] MAK Markwell et al. "A modification of the Lowry procedure to simplify protein determination in membrane and lipoprotein samples." In: Analytical biochemistry 87.1 (June 1978), pp. 206-210.

[33] Aneta J Mieszawska et al. "Engineering of lipid-coated PLGA nanoparticles with a tunable payload of diagnostically active nanocrystals for medical imaging." In: Chemical communications (Cambridge, England) 48.47 (June 2012), pp. 5835-7.

[34] Aneta J Mieszawska et al. "Multifunctional gold nanoparticles for diagnosis and therapy of disease." In: Molecular pharmaceutics 10.3 (Mar. 2013), pp. 831-47.

[35] Miho Oyasu et al. "Immunogold electron microscopic demonstration of distinct submembranous localization of the activated gammaPKC depending on the stimulation." In: The journal of histochemistry and cytochemistry : official journal of the Histochemistry Society 56.3 (Mar. 2008), pp. 253-65.

[36] René R S Packard and Peter Libby. "Inflammation in atherosclerosis: from vascular biology to biomarker discovery and risk prediction." In: Clinical chemistry 54.1 (Jan. 2008), pp. 24-38. 
[37] George Rouser, Sidney Fleischer, and Akira Yamamoto. "Two dimensional thin layer chromatographic separation of polar lipids and determination of phospholipids by phosphorus analysis of spots." In: Lipids 5.5 (May 1970), pp. 494-496.

[38] Andrea H Rowe et al. "Enhanced synthesis of the oxysterol $24(S), 25$-epoxycholesterol in macrophages by inhibitors of 2,3-oxidosqualene:lanosterol cyclase: a novel mechanism for the attenuation of foam cell formation." In: Circulation research 93.8 (Oct. 2003), pp. 717-25.

[39] J P Schlomka et al. "Experimental feasibility of multi-energy photon-counting K-edge imaging in pre-clinical computed tomography." In: Physics in medicine and biology 53.15 (Aug. 2008), pp. 4031-47.

[40] Matti M van Schooneveld et al. "A fluorescent, paramagnetic and PEGylated gold/silica nanoparticle for MRI, CT and fluorescence imaging." In: Contrast media $\mathcal{E}$ molecular imaging 5.4 (2010), pp. 231-6.

[41] J P Segrest et al. "Structure of apolipoprotein B-10o in low density lipoproteins." In: Journal of lipid research 42.9 (Sept. 2001), pp. 1346-67.

[42] Aviv Shaish et al. "Imaging of aortic atherosclerotic lesions by 125I-LDL, 125I-oxidized-LDL, 125I-HDL and 125I-BSA." In: Pathobiology 69.4 (Jan. 2002), pp. 225-229.

[43] H Sinzinger and H Bergmann. "Imaging of human atherosclerotic lesions using 123I-low-density lipoprotein." In: European journal of ... 12.5-6 (Jan. 1986), pp. 291-2.

[44] Liping Song et al. "Naphthalocyanine-reconstituted LDL nanoparticles for in vivo cancer imaging and treatment." In: International journal of nanomedicine 2.4 (Jan. 2007), pp. 767-74.

[45] Christian Steidl, Tang Lee, and SP Shah. "Tumor-associated macrophages and survival in classic Hodgkin's lymphoma." In: ... England Journal of . . 362.10 (Mar. 2010), pp. 875-85.

[46] Duxin Sun. "Nanotheranostics: integration of imaging and targeted drug delivery." In: Molecular Pharmaceutics 7.6 (Dec. 2010), p. 1879 .

[47] a J Versluis et al. "Receptor-mediated uptake of low-density lipoprotein by B16 melanoma cells in vitro and in vivo in mice." In: British journal of cancer 74.4 (Aug. 1996), pp. 525-532.

[48] Gang Zheng et al. "Low-density lipoprotein reconstituted by pyropheophorbide cholesteryl oleate as target-specific photosensitizer." In: Bioconjug. Chem. 13.3 (2002), pp. 392-396. 
[49] Gang Zheng et al. "Rerouting lipoprotein nanoparticles to selected alternate receptors for the targeted delivery of cancer diagnostic and therapeutic agents." In: Proceedings of the National Academy of Sciences of the United States of America 102.49 (Dec. 2005), pp. 17757-62. 



\title{
Comparison of Pharmaceutical Nanoformulations for Curcumin: Enhancement of Aqueous Solubil- ity and Carrier Retention ${ }^{1}$
}

Iris E. Allijn, Raymond M. Schiffelers, Gert Storm

\begin{abstract}
Curcumin, originally used in traditional medicine and as a spice, is one of the most studied and most popular natural products of the past decade. It has been described to be an effective anti-inflammatory and anti-cancer drug and protects against chronic diseases such as rheumatoid arthritis and atherosclerosis. Despite these promising pharmacological properties, curcumin is also very lipophilic, which makes its formulation challenging. Ideally the nanocarrier should additionally also retain the encapsulated curcumin to provide target tissue accumulation.

In this study we aimed to tackle this aqueous solubility and carrier retention challenge of curcumin by encapsulating curcumin in different nanoparticles. We successfully loaded LDL $(30 \mathrm{~nm})$, polymeric micelles $(80 \mathrm{~nm})$, liposomes $(180 \mathrm{~nm})$ and Intralipid $(280 \mathrm{~nm})$ with curcumin. The relative loading capacity was inversely related to the size of the particle. The stability for all formulations was determined in fetal bovine serum over a course of $24 \mathrm{~h}$.

Although all curcumin-nanoparticles were stable in buffer solution, all leaked more than $70 \%$ of curcumin under physiological conditions. Altogether, tested nanoparticles do solve the aqueous insolubility problem of curcumin, however, because of their leaky nature, the challenge of carrier retention remains.
\end{abstract}

1 Published in International Journal of Pharmaceutics, 2016, 206(2016), pp 407-413, DOI: 10.1016/j.ijpharm.2016.04.070 


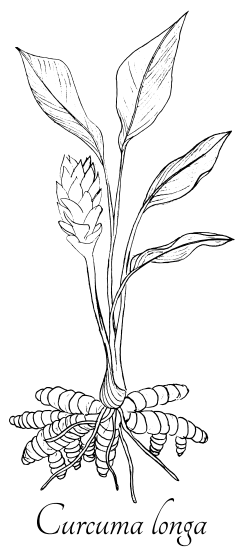

\subsection{INTRODUCTION}

\subsubsection{Curcumin}

Curcumin is isolated from Curcuma longa and used as a spice and in traditional medicine in Asia [16] and is together with quercetin and resveratrol among the most studied natural products. It has very potent pharmacological capabilities, exhibiting pleiotropic effects including the inhibition of TNF- $\alpha$, IL-1 $\beta$, IL-12, INF- $\gamma$, EGF, HGF, Stat3 and NF-k $\beta$ [16]. Furthermore, curcumin has been described to have anti-cancer, antiviral, anti-fungal, antioxidant, anti-angiogenic and anti-inflammatory properties [7] and shows activity in chronic diseases such as type 2 diabetes mellitus, rheumatoid arthritis, multiple sclerosis, Alzheimers' disease and atherosclerosis [16].

Despite the promising anti-inflammatory effects shown in an extensive collection of papers, there are some critical arguments concerning the real pharmacological actions of curcumin. Curcumin is one of the compounds that in drug screening assays easily gives positive hits, caused by its chemical structure rather than real pharmacological actions $[3,4]$. One of the reasons why curcumin is such a highly active compound and acts like a drug in most assays is the fact that it alters membrane properties. There is usually little evidence that curcumin has a direct interaction with a protein [8], which would make it a bona fide drug. Nevertheless, the non-specific membrane perturbations seem to be beneficial in a variety of - inflammation associated conditions in vivo, making curcumin an interesting pharmaceutical compound.

Of the physicochemical properties of curcumin (Figure 5.1) with a molecular weight $(\mathrm{MW})$ of 368.39 , lipophilicity $(\log \mathrm{D}$ at 7.4$)$ of 4.12 and a topological polar surface area (tPSA) of $93.06 \AA$, especially the low aqueous solubility stands out [11, 23]. Furthermore, in biological systems, curcumin is an unstable molecule, with rapid metabolism and degradation, for example into vanillin, and quick systemic elimination in vivo $[7,24]$. Additionally, curcumin has poor uptake after oral administration with about $75 \%$ direct elimination via the feces [24].

Altogether these highly unfavorable characteristics of curcumin ask for a solution to improve the bioavailability and insolubility. A potential solution are nanomedicine formulations. Indeed, curcumin has been encapsulated in a wide selection of nanocarriers to improve its solubility, but mainly to increase its bioavailability and protection against systemic degradation $[7,15,16]$. Ideally, curcumin would also be retained in the carrier to take advantage of the carrier's ability to target sites of disease. 


\subsubsection{Nanoparticle selection}

There is a variety of nanoparticles available that could potentially improve delivery and bioavailability of curcumin. Nanoparticles which are between 10 and $100 \mathrm{~nm}$ have the ability to take advantage of the enhanced permeability and retention (EPR) effect [17]. The EPR effect is the phenomenon of enhanced vascular endothelial permeability and impaired lymphatic drainage at inflammatory sites [13]. Nanoparticles passively diffuse through the endothelial lining and stay at the inflammatory site, because of poor lymphatic drainage, where they release their contents.

Often used, best known and FDA approved nanoparticles are the liposomes. These nanoparticles are typically 100-150 nm and consist of a phospholipid bilayer which can vary in composition, surrounding an aqueous core. Liposomes are suitable for hydrophilic compounds, which can reside in the core of the particle. Lipophilic compounds can nest in the fatty environment of the lipid bilayer, however, because of the dynamic nature of lipid exchange, drugs can easily be extracted. Liposomes are often the first of choice because of their ease of formulation, lipid composition tuning possibilities and the extensive experience with the system $[1,17]$.

Micelles are made from a single layer of amphiphilic molecules, such as phospholipids or polymers, surrounding a lipophilic core. These nanoparticles are particularly convenient for lipophilic pharmaceuticals because of their relatively high relative hydrophobic volume. An example of a micelle, however not often employed for drug delivery, is Intralipid. This micelle formulation is given as an intravenous nutrient. It is around $270 \mathrm{~nm}$ in diameter, readily available, cheap and FDA approved [12]. Therefore it is an undemanding starting point for delivery of lipophilic drugs.

Natural micelles which can be exploited as drug carriers are high density lipoprotein (HDL) and low density lipoprotein (LDL). The first one is a small $(\sim 10 \mathrm{~nm})$ micelle consisting of a single lysophospholipid

Please see chapter 4 for LDL as a drug carrier. layer and a lipophilic core. The particle is stabilized by the lipoprotein ApoA1. A mimic of this nanoparticle can be synthesized in the lab, as ApoAi easily attaches itself to the phospholipid layer. This nanoparticle is successfully used as drug carrier [6] and as contrast agent in<smiles>COc1cc(/C=C/C(=O)CC(=O)/C=C/c2ccc(O)c(OC)c2)ccc1O</smiles>

Figure 5.1: Molecular structure of curcumin. 
medical imaging [5]. LDL is slightly larger than HDL $(\sim 25 \mathrm{~nm})$ and also has a phospholipid monolayer with cholesterol and a lipophilic core containing cholesteryl esters and triglyceride [14]. The lipoprotein ApoBioo which is wrapped around the molecule is one of the largest known monomeric proteins [19] and is not able to be exchanged, as is the case for ApoAi. This makes the particle highly stable, and impossible to mimic in the lab. LDL has been used as a drug carrier though, by exchanging the core for a drug of choice using a core extraction method [10, 25] or sonication [2].

Polymeric micelles are especially designed for drug delivery and usually have a diameter below $100 \mathrm{~nm}$. Like liposomes, these micelles can be built from a variety of polymers and have been investigated extensively $[17,21]$. Often, these micelles suffer from instability in biological environments, due to exchange of the amphiphilic molecules with other surfaces. A solution to this exchange, is the covalent coupling of the micellar matrix within the core [18]. Still, these core-crosslinked micelles suffer from rapid release of the lipophilic payload which can be circumvented by also covalently linking these molecules to the covalently linked micelle matrix [22]. A recent advancement in micelle formulations circumventing the need for covalent crosslinking are the $\pi \pi$-stacking micelles [21]. These micelles derive their stability from the interactions between the $\pi$-orbitals of stacked aromatic rings. Since curcumin contains two aromatic rings it appears a suitable molecule to be employed in this strategy.

In this study, we compare four nanoparticles (Figure 5.2), namely liposomes, Intralipid, polymeric micelles and LDL as potential nanocarriers for their ability to enhance the aqueous solubility of curcumin and to retain the drug in the presence of plasma.
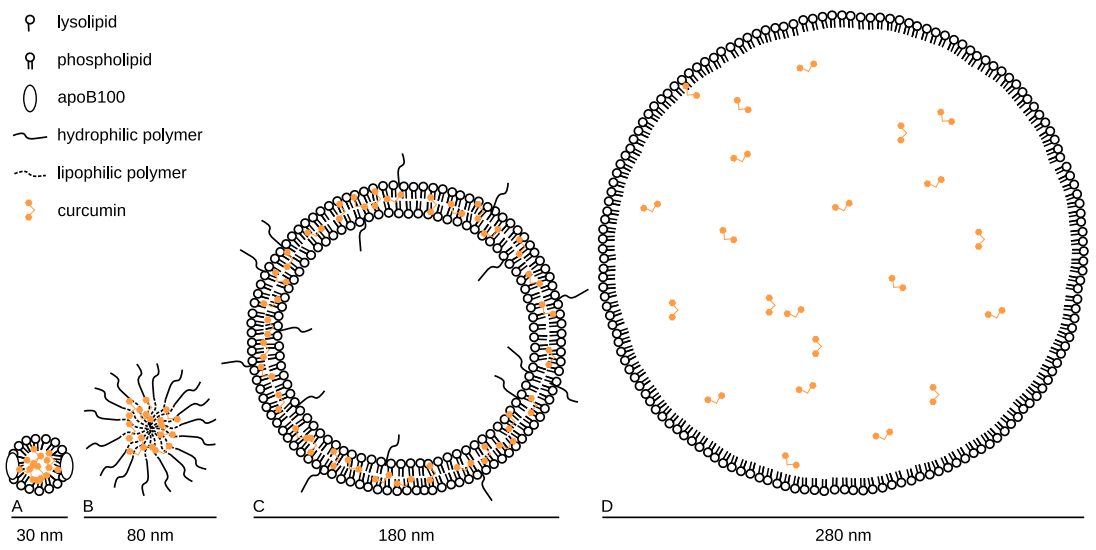

Figure 5.2: Schematic representation of curcumin loaded nanoparticles. A) LDL (cur-LDL), B) polymeric micelles (cur-mic), C) long circulating liposome (cur-lip), D) Intralipid (cur-intra). 


\subsection{RESULTS AND DISCUSSION}

\subsubsection{Diameter of curcumin nanoparticles}

The diameter of the cur-nanoparticles was determined using DLS (Figure 5.3 and Table 5.1). Control liposomes in HBS buffer measured a diameter of $110 \mathrm{~nm}$ with a pdi of 0.043 , whereas the diameter of curlip was $180 \mathrm{~nm}$ with a pdi of 0.105 . This increase of $60 \%$ in size and even larger increase of pdi for the cur-lips presumably reduces the ability of the liposomes to take advantage of the EPR effect. For this nanoparticles should ideally have a diameter between 10 and $100 \mathrm{~nm}$ [17]. However, an extra extrusion step through $0.1 \mu \mathrm{m}$ filters did not further reduce the size of these liposomes. It is possible that the curcumin inside the lipid bilayer prevented the increased lipid curvature.

Unmodified Intralipid had a diameter of $270 \mathrm{~nm}$ with a pdi of 0.124 , reducing its size by extrusion through 0.2 and $0.1 \mu \mathrm{m}$ filters to make it suitable to take advantage of the EPR effect did not lead to a size reduction but led to a marked decrease in number of particles and induced phase separation. Sonication for $1 \mathrm{~h}$ in a water bath did not affect the size of Intralipid. Cur-intra nanoparticles had a diameter of $280 \mathrm{~nm}$ with a pdi of 0.194 , which is only slightly larger than unloaded Intralipid. The increase in pdi indicates a less homogeneous particle populations after loading.

The natural (LDL) and synthetic micelles both displayed diameters which were well within the range for passive targeting using the EPR effect [17]. Unmodified LDL, had a diameter of $30 \mathrm{~nm}$ with a pdi of 0.272 which was unchanged for cur-LDL nanoparticles. Blank polymeric micelles had a diameter of $70 \mathrm{~nm}$ with a pdi of 0.121 and cur-mic was slightly larger with a diameter of $80 \mathrm{~nm}$ with a pdi of 0.093 . This is an increase in size of $11 \%$, however a decrease in polydispersity of $26 \%$.

Table 5.1: Size and curcumin concentration of cur-nanoparticles. pdi $=$ polydispersity index, Ratio $=$ relative cur $/$ nanoparticle ratio.

\begin{tabular}{lllll}
\hline & Diameter $(\mu \mathrm{m})$ & pdi & Curcumin $(\mathrm{mM})$ & Ratio \\
\hline Cur-lip & 0.18 & 0.105 & 2.4 & 0.101 \\
Cur-intra & 0.28 & 0.194 & 0.7 & 0.013 \\
Cur-LDL & 0.03 & 0.208 & 1.7 & 1.000 \\
Cur-mic & 0.08 & 0.090 & 9.6 & 0.438 \\
\hline
\end{tabular}




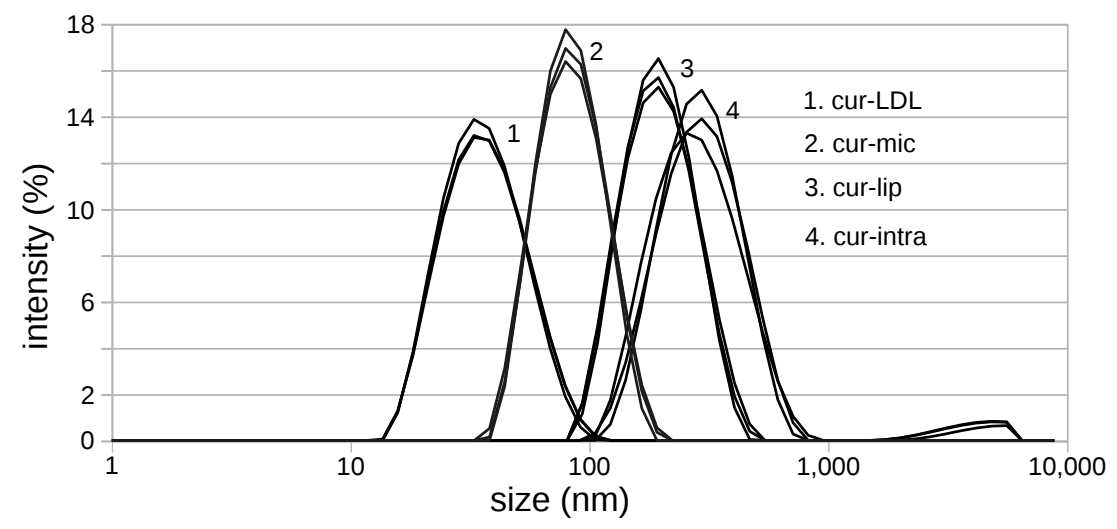

Figure 5.3: Cur-nanoparticle size distribution by intensity as measured by DLS.

\subsubsection{Curcumin concentration in curcumin nanoparticles}

Curcumin concentration in cur-nanoparticles was determined using UV-Vis at the wavelength for maximum absorbance of curcumin (429 $\mathrm{nm}$ ) and measured 2.4, 0.7, 1.7 and $9.6 \mathrm{mM}$ for cur-lip, cur-intra, curLDL and cur-mic respectively. When we determine the number of curnanoparticles and calculate their respective volume (assuming a perfect sphere, calculated using $1 / 6^{*} \pi^{*}$ diameter $^{3}$ ) and relate this to the curcumin concentrations, the loading capacity was shown to be inversely proportional to the volume of the particle (Table 5.1). It is interesting to note that the total curcumin loading is proportional to the surfacevolume ratio. This might indicate that the curcumin uptake into the particle over the particle interface is the rate limiting step. Alternatively, curcumin might have a preference of staying near the lipid membrane interface rather than moving to the core. It is surprising that cur-lip can accommodate a relatively large amount of curcumin despite the small lipophilic compartment. This supports the notion that the interface dictates the curcumin loading.

\subsubsection{Lipid profile in the curcumin-nanoparticles}

The lipid containing nanoparticles were characterized using a CAD coupled to a UPLC. Calibration curves of DSPE-PEG200o, cholesterol, DPPC and DSPC were included for comparison. Signals were detected at 7.9, 8.8, 9.3 and $10.0 \mathrm{~min}$ for respectively DSPE-PEG200o, cholesterol, DPPC and DSPC. For reference, a cur sample with a concentration of $0.5 \mathrm{mg} / \mathrm{mL}$ was included which showed a signal at $0.8 \mathrm{~min}$ (Figure $5 \cdot 4)$. 
Since cur-lip was prepared from the same lipids, the exact concentration could be calculated, using the lipid calibration curves, to be 9.9, 10.9 and $25.3 \mathrm{mg} / \mathrm{mL}$ for DPPC, DSPE-PEG2000, and cholesterol respectively. This translates into a molar ratio of $1: 0.10: 0.82$. The increase in molar ratio of cholesterol in the total composition compared to the feed ratio is significant. The loading of curcumin was $7 \%$ by molecular weight compared to DPPC.

For cur-intra and cur-LDL the composition of our particles is less well defined and consists of mixtures of phospholipids, cholesterol, cholesteryl esters and triglycerides. The phospholipids in these nanoparticles have similar structures as the reference DPPC and DSPC and indeed they show peaks with these retention times.

\subsubsection{Stability of cur-nanoparticles}

A critical characteristic of nanoparticles to take advantage of the EPR effect is to retain curcumin in the circulation. The retention of curcumin inside nanoparticles was tested by dialysis in FBS to mimic behavior in blood plasma. The temperature dependence of the process was tested by comparing $37{ }^{\circ} \mathrm{C}$ to $4{ }^{\circ} \mathrm{C}$. Macroscopically, all of the samples incubated at $37^{\circ} \mathrm{C}$, showed loss of yellow color, indicating that the intensely yellow-colored curcumin had escaped from the nanoparticle and entered the plasma (Figure 5.5). The diameter of all cur-nanoparticles was stable during the course of this stability experiment.

All cur-nanoparticles showed relative high leakage of curcumin at both temperatures. Cur-mic showed the highest leakage at $37{ }^{\circ} \mathrm{C}$, followed by cur-intra, cur-LDL and cur-lip (Table 5.2). This is contrary to expectations. Liposomes were not designed to be able to retain lipophilic drugs inside their bilayer. The PEG layer may have prevented interaction with plasma components, reducing extraction of the drug. For the micelles, we previously demonstrated that drugs containing aromatic groups, like paclitaxel, were stably retained within plasma in vivo. Apparently, other physicochemical characteristics of the molecules, beside aromatic groups, determine the extraction efficiency. Cur-lip and cur-mic both showed more leakage at $37^{\circ} \mathrm{C}$ incubated samples, whereas for cur-LDL this difference was minimal.

The size of the cur-nanoparticles in FBS after incubation and dialysis was determined as well. Cur-intra nanoparticles were the only of which the diameter at both temperatures was increased. The other type of curnanoparticles had reduced diameters in all incubation samples (Table $5 \cdot 2)$. 


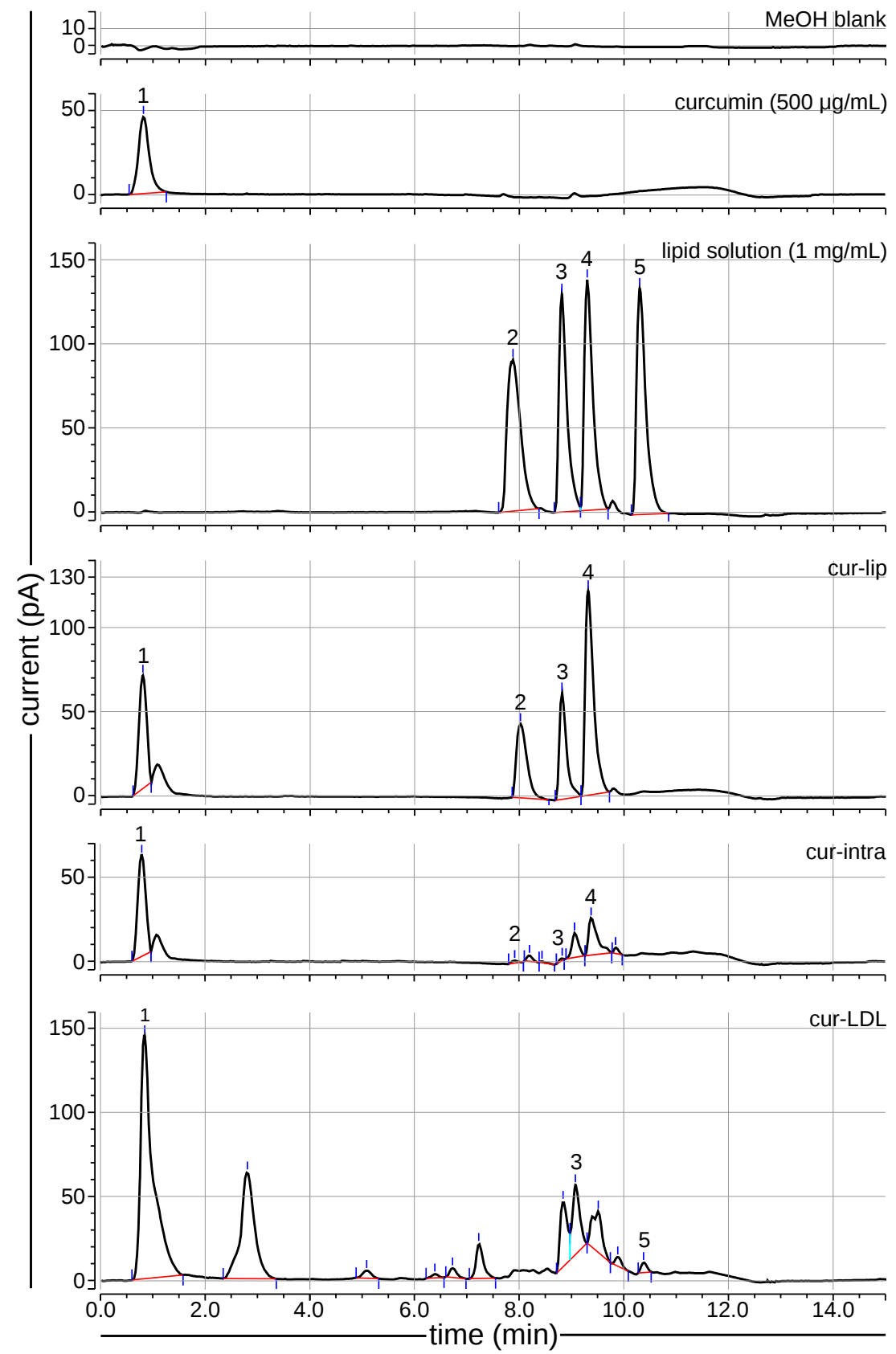

Figure 5.4: Lipid profiles of cur-nanoparticles. Lipid profiles of curnanoparticles were compared to a lipid mixture calibration curve containing DSPE-PEG2ooo (2), cholesterol (3), DPPC (4) and DSPC (5). A curcumin sample was included to identify the curcumin peak (1) in the cur-nanoparticles. 
Table 5.2: Curcumin concentration and size of cur-nanoparticles after incubation in FBS.

\begin{tabular}{|c|c|c|c|c|c|c|}
\hline & \multicolumn{2}{|l|}{$4^{\circ} \mathrm{C}$} & \multicolumn{2}{|l|}{$37^{\circ} \mathrm{C}$} & \multicolumn{2}{|c|}{$4{ }^{\circ} \mathrm{C}-37^{\circ} \mathrm{C}$} \\
\hline & $\mathrm{m} M$ & $\mu M$ & $\mathrm{mM}$ & $\mu M$ & $\mu M$ & $\mathrm{~nm}$ \\
\hline Cur-lip & $0.9(-62 \%)$ & $0.16(-12 \%)$ & $0.5(-78 \%)$ & $0.16(-13 \%)$ & $15 \%$ & $1 \%$ \\
\hline Cur-intra & $-(>-90 \%)$ & $0.31(+7 \%)$ & $-(>-90 \%)$ & $0.30(+6 \%)$ & - & $1 \%$ \\
\hline Cur-LDL & $0.3(-83 \%)$ & $0.03(-14 \%)$ & $0.2(-87 \%)$ & $0.03(-12 \%)$ & $5 \%$ & $3 \%$ \\
\hline Cur-mic & $1.8(-82 \%)$ & $0.06(-17 \%)$ & $0.6(-93 \%)$ & $0.05(-31 \%)$ & $12 \%$ & $16 \%$ \\
\hline
\end{tabular}

\section{$5 \cdot 3$ CONCLUSION}

All cur-nanoparticles function as solubilizers of curcumin in aqueous solutions, and make from this highly lipophilic compound an injectable drug. However, although the cur-nanoparticles are reasonably stable in buffer at $4{ }^{\circ} \mathrm{C}$, they do rapidly leak their curcumin payload in plasma at both 4 and $37^{\circ} \mathrm{C}$, and thus have limited opportunities to take advantage of the EPR effect.

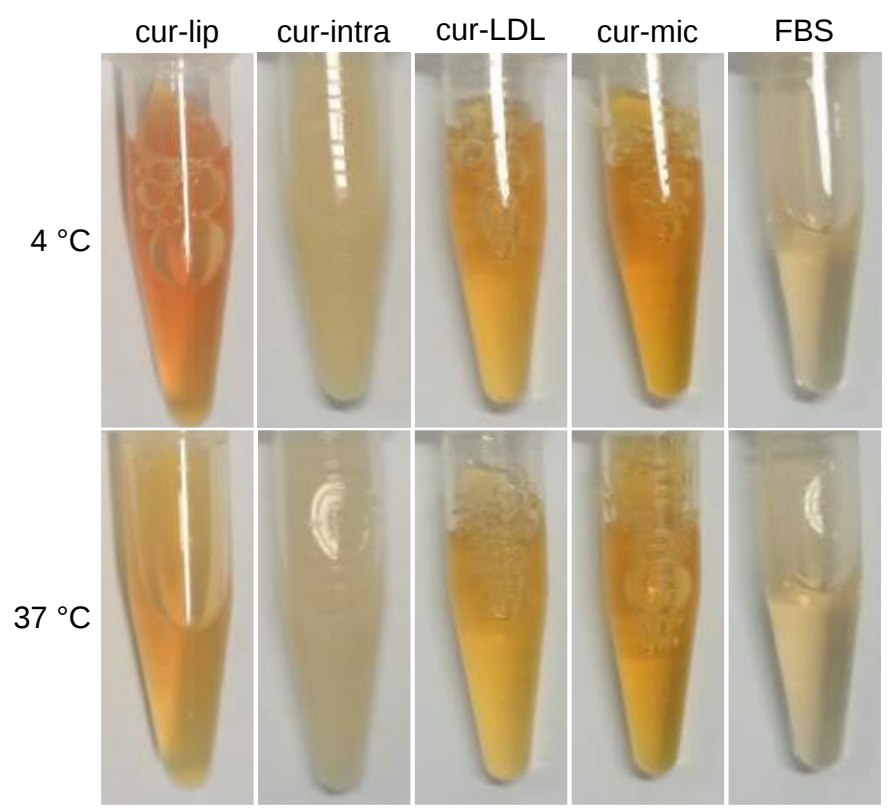

Figure 5.5: Cur-nanoparticle-FBS solutions after $24 \mathrm{~h}$ incubation and $24 \mathrm{~h}$ dialysis. 


\subsection{MATERIALS AND METHODS}

\subsubsection{Chemicals}

1,2-Dipalmitoyl-sn-glycero-3-phosphocholine (DPPC) and 1,2-Distearoyl-sn-glycero-3-phosphoethanolamine (DSPE-PEG 2000) and 1,2-distearoyl-sn-glycero-3-phosphocholine (DSPC) were purchased from Lipoid $\mathrm{GmbH}$, Germany. Cholesterol, methanol UPLC grade, tetrahydrofuran (THF) and triethylamine (TEA) were acquired from Sigma-Aldrich Chemie GmbH, Germany. Fetal bovine serum (FBS) was purchased from Lonza, Belgium, curcumin from Chengdu Biopurity Phytochemicals Ltd, ethanol absolute from Merck KGaA, Germany, HEPES from Acros Organics, Belgium, Intralipid from Fresenius Kabi Nederland BV, 1-Myristoyl-sn-glycero-3-phosphocho-line (MHPC) was purchased from Bachem, Germany and $\mathrm{NaCl}$ from Fisher Chemical, Fisher Scientific $\mathrm{GmbH}$, Germany. MilliQ water was obtained from a Merck Millipore Q-POD. mPEG-HPMA-Bz polymer was a kind gift from Aida Varela Moreira of Utrecht University.

\subsubsection{Curcumin property prediction and structure evaluation}

The molecular structure of curcumin was identified in SciFinder (Chemical Abstract Service (CAS) [20] and physicochemical properties were calculated by ChemAxon's Instant JChem [9].

\subsubsection{Formulation of curcumin-liposomes}

The liposomes were assembled with DPPC, DSPE-PEG 2000 and cholesterol in a molar ratio of 1:0.08:0.52. Lipids were placed in $1 \mathrm{~mL} \mathrm{EtOH}$ absolute and dissolved by applying heat. Maximum concentration of curcumin was dissolved in $\mathrm{EtOH}$ absolute and $1 \mathrm{~mL}$ of this solution was added to the lipid mixture. The curcumin-lipid solution in $\mathrm{EtOH}$ was rapidly added to $9 \mathrm{~mL}$ HBS buffer (10 mM HEPES with $150 \mathrm{mM}$ $\mathrm{NaCl}$ ) at $\mathrm{pH} 7.4$ using a preheated $5 \mathrm{~mL}$ syringe (BD Plastipak, Ireland) and a G18 needle (BD Microlance 3, Ireland) under constant heating and stirring. The orange-yellow solution was subsequently extruded using a thermostat pump at $70^{\circ} \mathrm{C}$ (Polystat 36, Fisher Scientific, The Netherlands) coupled to an extruder (Lipofast LF-50, Avastin, Germany). The sample was extruded using membranes (Nuclepore TrackEtch Membrane, Whatman) with pore sizes of $0.4,0.2$ and $0.1 \mu \mathrm{m}$. Curcumin liposomes (cur-lip) were dialyzed against HBS buffer $\mathrm{pH}$ 7.4 for about five days with three buffer changes. Any remaining free curcumin was removed from the liposomes using a Sephadex desalting column (PD10 column, GE Healthcare). Finally, cur-lips were filter 
sterilized using a $0.45 \mu \mathrm{m}$ filter (Merck Millipore Ltd, Ireland) and characterized. Control liposomes without curcumin were prepared in the same way.

\subsubsection{Formulation of curcumin-Intralipid}

Curcumin was added to Intralipid to a final concentration of $3 \mathrm{mg} / \mathrm{mL}$. The solution was vortexed well and subsequently sonicated in a water bath (Branson 2510) for $1 \mathrm{~h}$. To remove all unincorporated curcumin, the particles were cleaned using a Sephadex desalting column (PD10 column, GE Healthcare). The curcumin-Intralipid nanoparticles (curintra) were filter sterilized using a $0.45 \mu \mathrm{m}$ filter (Merck, MilliQ) and characterized.

\subsubsection{Formulation of curcumin-LDL}

Curcumin loaded LDL (cur-LDL) particles were prepared by a modified method as described before (Allijn2013). Briefly, cur micelles were formed using a 1:1 $(v / v)$ mixture of $20 \mathrm{mg} / \mathrm{mL}$ MHPC in EtOH absolute and $2 \mathrm{mg} / \mathrm{mL}$ curcumin in EtOH in a total volume of $1380 \mu \mathrm{L}$. The solution was drop-wise added to $5 \mathrm{~mL} 70{ }^{\circ} \mathrm{C}$ HBS buffer while stirring, to let the EtOH evaporate and form micelles. This turbid solution was subsequently purified using a $0.2 \mu \mathrm{m}$ filter (Spartan 13 syringe filter, Whatman) to remove curcumin aggregates. This cur-MHPC solution was then mixed with LDL in a 1:1 $(v / v)$ ratio and sonicated for $10 \mathrm{~min}$ in a water bath (Branson 2510). The solution was then con-

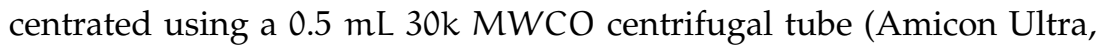
ultracel regenerated cellulose, Merck Millipore Ltd, Ireland). The clear go-through solution was discarded and the yellow solution in the filter unit was kept at $4{ }^{\circ} \mathrm{C}$ until further characterization.

\subsubsection{Formulation of curcumin-micelles}

Micelles were prepared from synthetic mPEG-HPMA-Bz polymers by dissolution in THF to get a final concentration of $5.6 \mathrm{mg} / \mathrm{mL}$. Curcumin was dissolved in THF as well and added under vigorously stirring to $\mathrm{mPEG}-\mathrm{HPMA}-\mathrm{Bz}$ in a 1:9 ratio resulting in a final concentration of $2.5 \mathrm{mg} / \mathrm{mL}$ curcumin and $5 \mathrm{mg} / \mathrm{mL}$ polymer. The solution was subsequently added drop wise to milliQ water in a 1:1 $(v / v)$ ratio and was left stirring for approximately $1 \mathrm{~min}$. After which it was left for $24 \mathrm{~h}$ in a fume hood to let the THF evaporate. After $24 \mathrm{~h}$, the micelles were spun down at $100 \mathrm{~g}$ for $1 \mathrm{~min}$ to get rid of the biggest curcumin aggregates and were subsequently filtered through a $0.45 \mu \mathrm{m}$ nylon filter (Acrodisc syringe filters, Pall Corporation). A total volume of curcumin 
micelles (cur-mic) of about $600 \mu \mathrm{L}$ was obtained. Following the same procedure, control micelles were prepared without curcumin.

\subsubsection{Nanoparticle size}

Size of the cur-nanoparticles was determined using dynamic light scattering (DLS) (Malvern Instruments). Samples were diluted until the solution was only slightly opalescent and measured at $20^{\circ} \mathrm{C}$, using a $173^{\circ}$ scattering angle. Intensity results were selected and the diameter and polydispersity index (pdi) of the nanoparticles was calculated by the machine's software (Zetasizer Software 7.02, Malvern).

\subsubsection{Curcumin concentration in curcumin-nanoparticles}

Curcumin concentration was determined using the UV-VIS function of a spectrophotometer (NanoDrop ND-100o spectrophotometer, Fisher Scientific, The Netherlands). A standard curve of curcumin was made of 1:2 serial dilution of curcumin in $\mathrm{EtOH}$. To dissolve the lipid part for no interference in the curcumin spectrum, cur-nanoparticles were diluted in $\mathrm{MeOH}$. Furthermore, solvent controls included water (blank), $\mathrm{HBS}$ buffer and $\mathrm{EtOH}$ and $\mathrm{MeOH}$. Absorption was measured at 429 $\mathrm{nm}$ and the concentration of the curcumin in the nanoparticles was deduced from the standard curve. FBS gave a background peak at 408 $\mathrm{nm}$, just next to the curcumin peak, and curcumin concentrations in the nanoparticles were corrected for this background.

\subsubsection{Stability of curcumin-nanoparticles in plasma}

Stability of the cur-nanoparticles in plasma was determined by incubation in FBS. $100 \mathrm{~mL}$ of cur-nanoparticles was added to $900 \mathrm{~mL}$ FBS and cur-nanoparticle-FBS solutions were incubated for $24 \mathrm{~h}$ at both 4 and $37^{\circ} \mathrm{C}$. After $24 \mathrm{~h}$, the solutions were dialyzed against FBS $(1: 14 \mathrm{v} / \mathrm{v})$ for $24 \mathrm{~h}$ at $4{ }^{\circ} \mathrm{C}$ using a $300 \mathrm{kDa}$ float-a-lyzer (spectra/por, Spectrum Laboratories, Inc.). Curcumin concentrations of the samples were determined using nanodrop and were used to determine the leakage of the nanoparticles.

\subsubsection{Lipid profiling of cur-nanoparticles}

The lipid content of the cur-nanoparticles was determined using a charged aerosol detector (CAD, Corona Ultra eso, Thermo Fisher/Dionex) coupled to a UHPLC device (Ultimate 3000, Thermo Fisher/Dionex), using a BEH C18 $1.7 \mathrm{~mm} 2.1 \times 50 \mathrm{~mm}$ column (Acquity, 
Waters, Ireland). A standard lipid curve containing DSPE-PEG 2000 , cholesterol, DPPC, and DSPC was prepared. A gradient run was used with mobile phase $A=80 \% \mathrm{MeOH}$ and $0.1 \%$ TEA in milliQ water and mobile phase $\mathrm{B}=100 \% \mathrm{MeOH}$ and $0.1 \%$ TEA. Mobile phases were degassed using a water bath sonicator (Branson 2510) for $5 \mathrm{~min}$. The UHPLC had a runtime of $15 \mathrm{~min} / \mathrm{sample}$ at a flow of $0.200 \mathrm{~mL} / \mathrm{min}$. The gradient was set as follows: $0.0-0.890 \mathrm{~min}=100 \% A, 2.0-7.56 \mathrm{~min}$ $=100 \% \mathrm{~B}, 7.840-15.00 \mathrm{~min}=100 \%$ A. Injection volume was $4 \mathrm{~mL}$ and column temperature was set to $50{ }^{\circ} \mathrm{C}$. Lipids were detected with peaks at 8, 8.9, 9.4 and $10.4 \mathrm{~min}$ respectively for DSPE-PEG200o, cholesterol, DPPC and DSPC using the CAD. Lipid profiles were compared to the known lipids and concentrations in the liposomes were determined from a quadratic calibration curve. Concentrations were calculated using the accompanying software (Chromeleon Chromatography Data System version 7.1.2.1713).

\section{Acknowledgment}

NanoNextNL grant 03D.11 (R.M. Schiffelers) and mPEG-HPMA-Bz polymer was a kind gift from Aida Varela Moreira of Utrecht University. Acarilia Eduardo da Silva kindly helped optimizing the CADUHPLC method for lipid detection.

\section{REFERENCES}

[1] Theresa M. Allen and Pieter R. Cullis. "Liposomal drug delivery systems: From concept to clinical applications." In: Advanced Drug Delivery Reviews 65.1 (2013), pp. 36-48.

[2] Iris E Allijn et al. "Gold nanocrystal labeling allows low-density lipoprotein imaging from the subcellular to macroscopic level." In: ACS nano 7.11 (Nov. 2013), pp. 9761-70.

[3] Jonathan B. Baell. "Screening-based translation of public research encounters painful problems." In: ACS Medicinal Chemistry Letters 6.3 (2015), pp. 229-234.

[4] Jonathan B. Baell and Michael A. Walters. "Chemical con artists foil drug discovery." In: Nature 513 (2014), pp. 481-483.

[5] David P. Cormode et al. "HDL as a contrast agent for medical imaging." In: Clinical lipidology 4.4 (Aug. 2009), pp. 493-500.

[6] Raphaël Duivenvoorden et al. "A statin-loaded reconstituted high-density lipoprotein nanoparticle inhibits atherosclerotic plaque inflammation." In: Nature Communications 5 (2014), pp. 1-12. 
[7] Gagan Flora, Deepesh Gupta, and Archana Tiwari. "Nanocurcumin: a promising therapeutic advancement over native curcumin." In: Critical reviews in therapeutic drug carrier systems 30.4 (2013), pp. 331-368.

[8] Helgi I. Ingólfsson et al. "Phytochemicals perturb membranes and promiscuously alter protein function." In: ACS Chemical Biology 9.8 (2014), pp. 1788-1798.

[9] Instant JChem Suite, v15.8.17.0. 2015. URL: http ://www . chemaxon . com.

[10] Monty Krieger. "Reconstitution of the hydrophobic core of low-density lipoprotein." In: Methods in enzymology 128.1977 (Jan. 1986), pp. 6o8-13.

[11] Christopher A. Lipinski et al. "Experimental and computational approaches to estimate solubility and permeability in drug discovery and development setting." In: Advanced Drug Delivery Reviews 46.1-3 (2001), pp. 3-26.

[12] Fresenius Kabi Ltd. Intralipid - product monograph. 2015.

[13] Y. Matsumura and H Maeda. "A new concept for macromolecular therapeutics in cancer chemotherapy: mechanism of tumoritropic accumulation of proteins and the antitumor agent smancs." In: Cancer research 46.12 Pt 1 (Dec. 1986), pp. 6387-92.

[14] J. R. McNamara et al. "Differences in LDL subspecies involve alterations in lipid composition and conformational changes in apolipoprotein B." In: Journal of Lipid Research 37.9 (Sept. 1996), pp. 1924-1935.

[15] Chandana Mohanty, Manasi Das, and Sanjeeb K Sahoo. "Emerging role of nanocarriers to increase the solubility and bioavailability of curcumin." In: Expert Opinion on Drug Delivery 9.11 (2012), pp. 1347-1364.

[16] Ornchuma Naksuriya et al. "Curcumin nanoformulations: a review of pharmaceutical properties and preclinical studies and clinical data related to cancer treatment." In: Biomaterials 35.10 (Mar. 2014), pp. 3365-3383.

[17] Robby A. Petros and Joseph M. DeSimone. "Strategies in the design of nanoparticles for therapeutic applications." In: Nature reviews. Drug discovery 9.8 (Aug. 2010), pp. 615-27.

[18] Cristianne J. Rijcken et al. "Hydrolysable core-crosslinked thermosensitive polymeric micelles: Synthesis, characterisation and in vivo studies." In: Biomaterials 28.36 (2007), pp. 5581-5593.

[19] J P Segrest et al. "Structure of apolipoprotein B-10o in low density lipoproteins." In: Journal of lipid research 42.9 (Sept. 2001), pp. $1346-67$. 
[20] Chemical Abstract Service. SciFinder. 2013. URL: https : //scifinder.cas.org.

[21] Yang Shi et al. "П-П Stacking increases the stability and loading capacity of thermosensitive polymeric micelles for chemotherapeutic drugs." In: Biomacromolecules 14.6 (2013), pp. 1826-1837.

[22] Marina Talelli et al. "Core-crosslinked polymeric micelles with controlled release of covalently entrapped doxorubicin." In: Biomaterials 31.30 (2010), pp. 7797-7804.

[23] Daniel F. Veber et al. "Molecular properties that influence the oral bioavailability of drug candidates." In: Journal of medicinal chemistry 45.12 (2002), pp. 2615-2623.

[24] Ying J. Wang et al. "Stability of curcumin in buffer solutions and characterization of its degradation products." In: Journal of Pharmaceutical and Biomedical Analysis 15.12 (1997), pp. 1867-1876.

[25] Gang Zheng et al. "Low-density lipoprotein reconstituted by pyropheophorbide cholesteryl oleate as target-specific photosensitizer." In: Bioconjug. Chem. 13.3 (2002), pp. 392-396. 



\section{BERBERINE}

\section{Liposome encapsulated berberine treatment attenuates cardiac dysfunction after myocardial infarction $^{1}$}

Iris E. Allijn, Bertrand M.S. Czarny ${ }^{2}$, Xiaoyuan Wang, Suet Y. Chong, Marek Weiler, Acarilia Eduardo da Silva, Josbert M. Metselaar, Giorgia Pastorin, Dominique P.V. de Kleijn, Gert Storm, Jiong-Wei Wang, Raymond M. Schiffelers

\section{ABSTRACT}

Adverse left ventricular remodeling can be evaluated as a reduction in ejection fraction after myocardial infarction (MI). This leads to heart failure and is a main determinant of mortality and morbidity after MI. To improve remodeling, anti-inflammatory compounds have shown promise. Berberine has ascribed anti-inflammatory, anti-oxidative, and cardioprotective properties. However, the poor solubility in aqueous buffers and a short half-life in the circulation have been impeding the usage of this natural product. We hypothesize that encapsulation of berberine into long circulating liposomes could improve its therapeutic availability and efficacy to protect cardiac function in vivo. Berberine loaded liposomes $(0.3 \mathrm{mg} / \mathrm{mL}$ and $0.11 \mu \mathrm{m}$ in diameter $)$ were prepared and characterized. Subsequently they were tested for IL-6 secretion inhibition in RAW 264.7 macrophages and for cardiac function protection against $\mathrm{MI}$ in $\mathrm{C}_{57} \mathrm{BL} / 6 \mathrm{~J}$ mice. In vitro, free berberine significantly inhibited IL-6 secretion $\left(\mathrm{IC}_{50}=10.4 \mu \mathrm{M}\right)$, whereas encapsulated berberine did not. In vivo, however, berberine loaded liposomes did significantly preserve the cardiac ejection fraction at day 28 after MI by $64 \%$ compared to control liposomes and free berberine. In conclusion, liposomal encapsulation enhanced the solubility of berberine in buffer and improved cardiac protective effects of berberine against MI. This outcome indicates that delivery of berberine via liposomes significantly

1 Manuscript submitted

2 Shared first author 
improves its therapeutic availability and therefore treatment efficacy in vivo.

\subsection{INTRODUCTION}

Acute myocardial infarction (MI) as a result of coronary artery occlusion can induce adverse left ventricular remodeling, which leads to chronic congestive heart failure (CHF) [16]. CHF is a chronic disease in which the heart cannot supply enough blood and thus oxygen and pressure to the organs. This results in severe fatigue, breathlessness and salt-water retention in the kidneys, which in turn can lead to kidney failures [15]. Despite advances in the treatment of CHF over the last decades, it remains one of the major causes of death worldwide [9]. Left ventricular remodeling is a complex process involving inflammation and infiltration of innate immune cells, in particular macrophages, into the insulted heart tissue [4]. Although it is generally accepted that inflammation plays crucial roles in adverse left ventricular remodeling and subsequently CHF, there is no evidence-based therapy focused on reducing inflammation yet.

Berberis spp. are plants from the Berberidaceae family [12]. These plants are widely distributed over and known around the world [10]. It is one of the many plants from which berberine, a small fluorescent isoquinoline quarternary alkaloid $[17,20]$ (Figure 6.1), can be isolated [10, 20]. Both berberine and the plant have a long history in Middle Eastern [11], Ayurvedic [20], Chinese [12] and Native American [17] traditional medicine. Berberine has been indexed in the Medical Subject Headings (MeSH) since 1975 [14] and nowadays has extensive attention from the Western scientific community [20]. Beneficial properties such as antiinflammatory, anti-microbial, anti-diarrheal [17] anti-oxidative [17, 8], vasorelaxant [17, 11] and cholesterol lowering [11] effects have been ascribed to this natural product.

Importantly, long term and high dose berberine treatment has been reported to improve cardiac function [9, 12] and to exert cardioprotective effects [24] in murine heart failure models. Furthermore, berberine was shown to improves survival in patients with CHF [23]. However, clinical use of berberine has been greatly impeded by its limited bioavailability due to the low absorption rate in the intestine (less than $5 \%$ ) and excretion by P-glycoprotein and multidrug resistance associated protein-1 [18].

In this study we investigated the use of berberine to reduce CHF and protect heart function in a mouse MI model by ligation of the left anterior descending artery. To overcome the low bioavailability of free berberine $(\mathrm{BBCl})$ in the affected heart, berberine was loaded in long 
circulating liposomes (BBCl-lip) and administered intravenously. Due to enhanced vascular permeability [6], liposomes are expected to passively target the inflammatory site and subsequently release the drug after uptake by macrophages present in the region. This process would achieve to have better targeting and pharmacokinetics improving therapeutic effect and could decrease berberine exposure, reducing potential adverse effects.<smiles>COc1ccc2cc3[n+](cc2c1OC)CCc1cc2c(cc1-3)OCO2</smiles>

Figure 6.1: Molecular structure of berberine chloride.

\subsection{RESULTS AND DISCUSSION}

\subsubsection{Size of liposomes}

DLS measurements on BBCl-lip showed an average diameter of 0.11 $\mu \mathrm{m}$ with a polydispersity index of 0.048 , which indicates a homogeneous particle solution. The control liposomes were with a diameter of $0.11 \mu \mathrm{m}$ and a polydispersity index of 0.043 , very comparable in both size and distribution (Table 6.1). Over a period of two years, the diameter of the BBCl-lip did not significantly change, demonstrating the stable nature of the liposomes when kept at $4{ }^{\circ} \mathrm{C}$ (Figure 6.2).

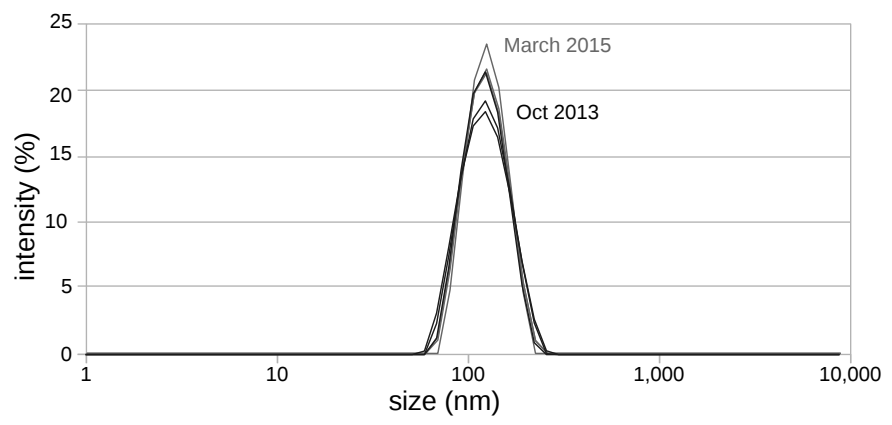

Figure 6.2: Particle size distribution of BBCl-lip. Measurements performed in October 2013 compared to measurements in March 2015 on the same samples. 
6.2.2 $\mathrm{BBCl}$ concentration in liposomes is higher than the saturated concentration of free $\mathrm{BBCl}$ in buffer

A BBCl content of $0.3 \mathrm{mg} / \mathrm{mL}$ for the $\mathrm{BBCl}$-lip was determined using UV-VIS measurements. Of the initial $2.9 \mathrm{mg} / \mathrm{mL}$ berberine solution in HBS, only $10.4 \%$ was incorporated. The remainder was lost during extrusion and/or purification of the liposomes. $\mathrm{BBCl}$ could only be entirely dissolved at a concentration of $2.9 \mathrm{mg} / \mathrm{mL}$ in buffer at $70^{\circ} \mathrm{C}$, the temperature at which the extrusion took place. As soon as the solution cooled down, $\mathrm{BBCl}$ started precipitating.

At a concentration of $0.3 \mathrm{mg} / \mathrm{mL} \mathrm{BBCl}$ was not completely dissolved at RT. In liposomes, the concentration of $0.3 \mathrm{mg} / \mathrm{mL} \mathrm{BBCl}$ in was maintained even at $4{ }^{\circ} \mathrm{C}$. The fact that $\mathrm{BBCl}$ poorly dissolves in buffer solutions, underlines the importance of the introduction of a nanocarrier like $\mathrm{BBCl}-$ lip for this natural product. $\mathrm{BBCl}$ could be dissolved at a concentration of $0.3 \mathrm{mg} / \mathrm{mL}$ by warming to $37^{\circ} \mathrm{C}$. This is of importance for the in vivo experiments performed in this study as this allowed to inject equal doses with equal injection volumes.

\subsubsection{Lipid composition of BBCl-lip}

The lipid composition of BBCl-lip was determined using UPLC-CAD and a lipid mixture calibration standard containing the same lipids as used in the preparation of BBCl-lip (Figure 6.3). In BBCl-lip we recovered 57.8, 18.01 and $15.2 \mathrm{mg} / \mathrm{mL}$ for respectively DPPC, DSPEPEG200o and cholesterol, which is a molar ratio of $1: 0.08: 0.50$. This is similar for what we recovered in the blank liposomes (Table 6.1), making these liposomes a suitable control. The molar ratio of the lipids in the liposomes shows that the liposomes become relatively enriched with cholesterol compared to the feed ratio.

Table 6.1: Liposome characteristics. The diameters including the pdi and the concentrations of the lipids and $\mathrm{BBCl}$ with molar ratio to DPPC are given. Both liposomes are very similar in both composition and size.

\begin{tabular}{llllll}
\hline Characteristics & BBCl-lip & Ratio & Control-lip & Ratio & Feed ratio \\
\hline Diameter $(\mu \mathrm{m})$ & 0.11 & & 0.11 & & \\
pdi & 0.048 & & 0.043 & & \\
DPPC $(\mathrm{mg} / \mathrm{mL})$ & 57.8 & 1 & 60.2 & 1 & 1 \\
DSPE-PEG $2000(\mathrm{mg} / \mathrm{mL})$ & 18.1 & 0.08 & 17.7 & 0.08 & 0.08 \\
Cholesterol $(\mathrm{mg} / \mathrm{mL})$ & 15.2 & 0.50 & 15.7 & 0.50 & 0.28 \\
$\operatorname{BBCl}(\mathrm{mg} / \mathrm{mL})$ & 0.3 & 0.01 & & & \\
\hline
\end{tabular}




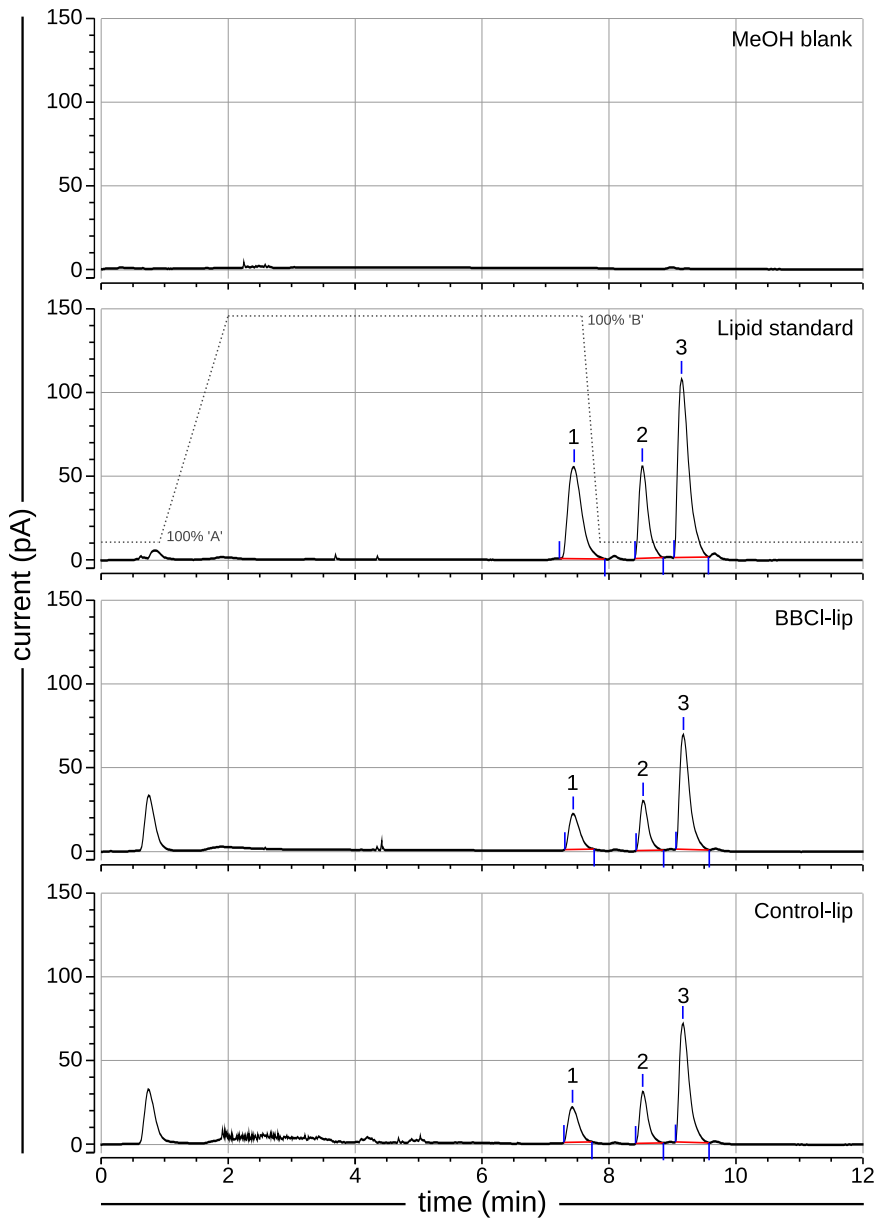

Figure 6.3: Lipid profile of BBCl-lip. Lipid composition of BBCl-lip was determined using a lipid mixture calibration standard (highest standard shown) containing 1) DSPE-PEG 2000 , 2) cholesterol and 3) DPPC. The solvent gradient was included in the chromatogram of the lipid standard with ' $\mathrm{A}$ ' $=80 \% \mathrm{MeOH}$ with $0.1 \%$ TEA and ' $\mathrm{B}$ ' $=$ $100 \% \mathrm{MeOH}$ with $0.1 \%$ TEA. 
6.2.4 BBCl-lip does not inhibit pro-inflammatory cytokine IL-6 secretion in vitro

As we have previously shown, free berberine inhibits IL-6 cytokine expression in RAW 246.7 macrophages $\left(\mathrm{IC}_{50}=10.4 \mu \mathrm{M}, \mathrm{E}_{\max }=44 \%\right.$ and $\mathrm{R}^{2}=0.77$, Chapter 3, Table 3.3)[3]. Inhibition of IL-6 by BBCl-lip, following the same protocol, showed no concentration dependent inhibition (Figure 6.4). These results show that in vitro the liposomal formulation does not increase the effectiveness of $\mathrm{BBCl}$. This is likely due to the fact that long circulating PEGylated liposomes are designed to have a prolonged circulation time in vivo by avoiding cell interaction. This is beneficial in vivo since it enhances the accumulation of drug-loaded liposomes at the desired tissue. However, the reduced cellular uptake in in vitro settings reduces efficacy.

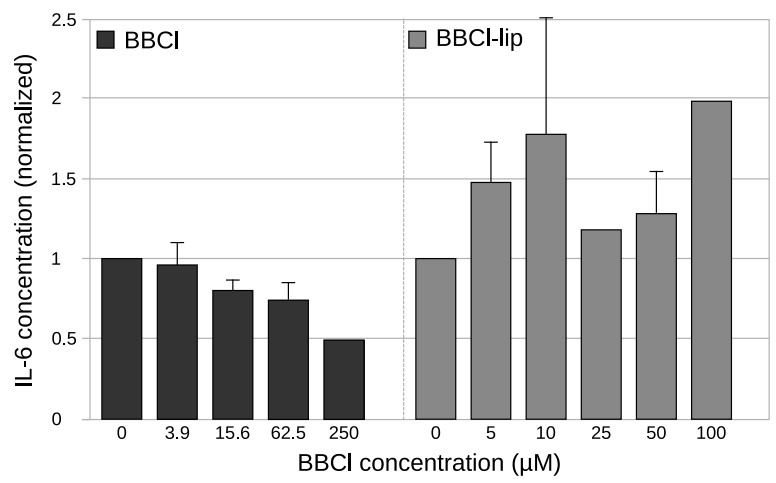

Figure 6.4: In vitro IL-6 expression levels after incubation with $\mathrm{BBCl}$ or BBCl-lip in RAW 264.7 macrophages. Incubation with $\mathrm{BBCl}$ showed a dose dependent response with an $E_{\max }$ of $44 \%$, a curve fit of $R^{2}$ of 0.77 and an $\mathrm{IC}_{50}$ of $10.4 \mu \mathrm{M}$. Incubation with BBCl-lip did not result in a concentration dependent inhibition. Bars represent means + SEM.

\subsubsection{Cy5.5-lip accumulates in myocardial infarction}

Macrophages are known to infiltrate into the infarcted heart tissue from circulation (Fig 6.5A-B) [21]. Since macrophages have been reported to take up liposomes and to release the preloaded drug in vivo (REF??), we hypothesized that encapsulation of berberine into liposomes would enrich the berberine in the infarcted heart tissue and therefore improve its local delivery and treatment efficacy. To prove this concept, we directly visualized the distribution of liposomes in the heart by injecting liposomes containing Cy5.5 fluorescent dye in mice. As expected, the liposomes accumulated specifically in the infarcted 
heart tissue ( 3 days after MI) and likely in the infiltrated macrophages (Fig 6.5C-D). Liposomes were also observed in the heart 1 day and 7 days after MI (data not shown). These results indicate that liposomes are able to facilitate local delivery of specific drugs such as berberine into the insulted heart tissue.
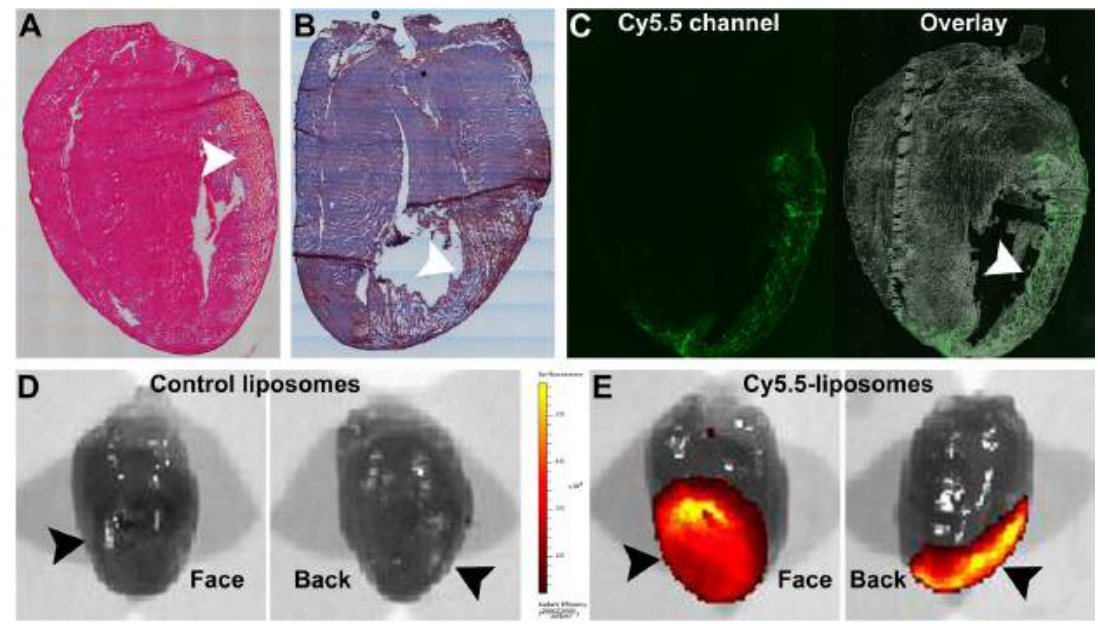

Figure 6.5: Infiltration of macrophages and accumulation of liposomes in the infarcted heart tissue. Left ventricles were isolated 3 days after MI and analyzed for liposomes and macrophages. A) H\&E staining to indicate the infarcted area. B) Infiltration of macrophages into the infarcted heart tissue. MAC 3 is shown in brown. C) Liposomes visualized with confocal microscopy. Cy5.5 is shown in green and merged with heart tissue obtained under bright field. D) Accumulation of control liposomes in the infarcted tissue visualized with IVIS spectrum imaging system. E) Accumulation of Cy5.5-liposomes in the infarcted tissue visualized with IVIS spectrum imaging system.

\subsubsection{BBCl-lip protects heart function against myocardial infarction}

Mouse heart function was evaluated by cardiac left ventricular ejection fraction (LVEF). At day 28 after MI, BBCl-lip significantly preserved LVEF, while free $\mathrm{BBCl}$ did not show any effect compared to control-lip $(29.5 \pm 1.9$ with $p<0.05$ for BBCl-lip, $18.2 \pm 3.2 \%$ for $\mathrm{BBCl}$ and 18.0 $\pm 3.1 \%$ for control-lip). Furthermore, hypertrophy of the left ventricle was less severe, though not statistically significant, in mice treated with $\mathrm{BBCl}$-lip (Figure 6.6). These results indicate that the delivery of $\mathrm{BBCl}$ by long circulating liposomes via intravenous injection efficiently reduced cardiac adverse remodeling and hence attenuated heart dysfunction in mice subjected to MI. 
Left ventricular ejection fraction

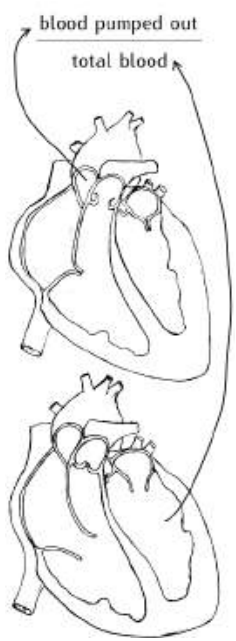

Berberine has shown therapeutic efficacy in CHF patients [23] and in animal models of MI [24] or ischemic reperfusion injury [9, 5]. In these studies, berberine was given orally or intraperitoneally in long term high concentrations (3 - 66 times higher than our dosage). In animal studies, berberine has mostly been given before MI induction, however, in practice, patients are treated after the diagnosis of MI. To mimic the clinical situation, we have chosen to treat our mice after the onset of MI.

In our mouse MI model, the first week is dominated by the inflammatory response, including the production of reactive oxygen species, inflammatory cell infiltration (like macrophages) in the heart and subsequently cytokine expression $[7,4,22]$. To reduce the inflammatory damage after MI and hence protect heart function, we ensured delivery of $\mathrm{BBCl}$ to the local inflammatory environment via long-circulating liposomes. Since the blood circulation half-life of PEGylated liposomes is around $16-24 \mathrm{~h}$ in mice [2, 1, 19], additional injections on day 3 and day 6 after MI ensured continued presence of $\mathrm{BBCl}$ during this first week. The therapeutic effects in these conditions resulted in a significant improvement 28 days after MI compared to the delivery of control-lip and $\mathrm{BBCl}$. In this manner, we have dramatically augmented the treatment efficacy of berberine and improved cardiac function by $64 \%$ compared to the same dose of free $\mathrm{BBCl}$ (Figure 6.6). This is a promising first step for translation to the clinic.

\subsection{CONCLUSIONS}

Encapsulation of berberine into liposomes is crucial for improvement of solubility in aqueous buffers and enhances the therapeutic availability of this natural product. Intravenous administration via liposome significantly improved the treatment efficacy of berberine to protect cardiac function after MI.

\subsection{MATERIALS AND METHODS}

\subsubsection{Chemicals}

Berberine chloride form, Lipopolysaccharides from Escherichia coli 055:B5, cholesterol and penicillin/streptomycin, sulfuric acid, triethylamine, metha-nol LC-MS grade (Fluka Analytical) and resazurin sodium salt were purchased from Sigma-Aldrich Chemie BV, The Netherlands. Albumin bovine fraction V, sodium chloride and 1-step ultra TMB ELISA substrate were obtained from Fisher Chemical, Thermo 
Scientific, BV, The Netherlands. IL-6 cytoset was acquired from Life Technologies BV, The Netherlands. Prednisolone disodium phosphate was obtained from Fagron BV, The Netherlands. Tween 20 and HEPES were purchased from Acros Organics, Belgium. RPMI-1640 without Lglutamine and L-glutamine $200 \mathrm{mM}$ from PAA Laboratories $\mathrm{GmbH}$, Germany. Fetal bovine serum (FBS) was purchased from Lonza, Belgium, dipalmitoylphosphatidylcholine (DPPC) and distearoylphosphoethanolamine-poly ethylene glycol 2000 (DSPE-PEG 2000 ) were obtained from Lipoid GmbH, Germany. Ethanol absolute, ACS, ISO, Reag. Ph Eur was purchased from Merck Millipore B.V., Amsterdam, The Netherlands. MilliQ water was obtained from a Merck Millipore QPOD. Medetomidine and atipamezole were purchased from Phizer Animal Health, Exton, PA, USA. Dormicum was purchased from sciencelab.com, Texas, USA, fentanyl from Phizer Pharmaceuticals Group, New York, USA, flumazenil from Sagent Pharmaceuticals, Illinois, USA, Temgesic from Hospira Inc., Illinois, USA, and isoflurane from Baxter, Singapore.

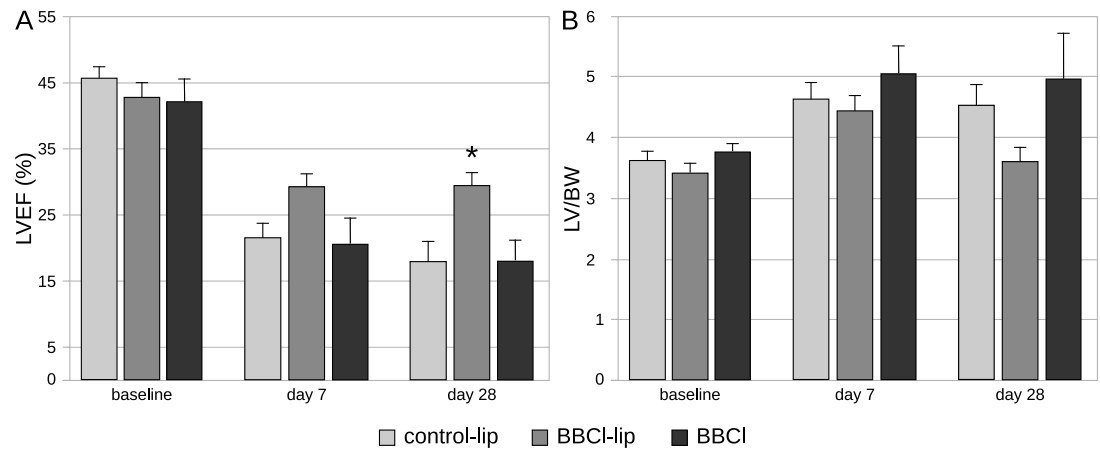

Figure 6.6: Cardiac function and remodeling after MI. Mice are treated with control liposomes (control-lip, $\mathrm{n}=10$ ), lipsome encapsulated berberine (BBCl-lip, $n=10$ ) or free berberine $(\mathrm{BBCl}, \mathrm{n}=6)$. A) Cardiac LVEF was determined by echocardiography at baseline, 7 days and 28 days after MI surgery, ${ }^{*} p<0.05$ for BBCl-lip, free BBCl did not preserve the ejection fraction compared to control-lip. B) Left ventricular mass estimated by echocardiography at baseline, 7 days and 28 days after MI surgery. Two-way ANOVA with Bonferroni post hoc was performed for multiple comparisons. Bars represent mean + SEM. LVEF = left ventricle ejection fraction, $\mathrm{LV} / \mathrm{BW}=\mathrm{left}$ ventricle weight per body weight. 


\subsubsection{BBCl-lip preparation}

Liposomes were prepared by the 'ethanol injection method'. Briefly, $734.1 \mathrm{mg}$ DPPC, $226.0 \mathrm{mg}$ DSPE-PEG 2000 and $209.0 \mathrm{mg}$ cholesterol (molar ratio: $1: 0.08: 0.28$ ) were dissolved in $1 \mathrm{~mL}$ ethanol absolute while stirring on a hot plate. $\mathrm{BBCl}$, with a final concentration of $\sim 3$ $\mathrm{mg} / \mathrm{mL}$, was dissolved in $9 \mathrm{~mL}$ HEPES buffered saline (HBS, $10 \mathrm{mM}$ HEPES with $150 \mathrm{mM} \mathrm{NaCl}$ ) at $\mathrm{pH} 7.4$ while stirring on a hot plate. Once both solutions were clear, the lipids were rapidly injected into the $\mathrm{BBCl}$ solution using a preheated $5 \mathrm{~mL}$ syringe (BD Plastipak, Ireland) and G18 1 1/2 needle (BD Microlance 3, Ireland). The BBCl-lipid solution was extruded multiple times at $70{ }^{\circ} \mathrm{C}$ with a final membrane size of $0.1 \mu \mathrm{m}$ (Nuclepore Track-Etch Membrane, Whatman) using a thermostat pump (Polystat 36, Fisher Scientific, The Netherlands) coupled to an extruder (Lipofast LF-50, Avastin, Germany). Subsequently, the BBCl-lip solution was dialyzed against HBS buffer at $\mathrm{pH} 7.4$ for two days, changing buffer four times, to remove ethanol and free $\mathrm{BBCl}$. To remove the final free $\mathrm{BBCl}, \mathrm{BBCl}$-lips were cleaned using a PDio Sephadex G-25M column (GE Healthcare, UK). BBCl-lips were stored at $4{ }^{\circ} \mathrm{C}$ until further usage. Control liposomes (control-lip) without $\mathrm{BBCl}$ were prepared in the same way. Cyanine 5.5 liposomes (Cy5.5lip) were prepared as described by Lobatto et al. [13].

\subsubsection{Size determination of BBCl-lip}

The diameter of the BBCl-lip was determined using dynamic light scattering (DLS, Malvern Instruments). Samples were diluted until the solution was only slightly yellow and measured at $20^{\circ} \mathrm{C}$ using a $173^{\circ}$ scattering angle. Intensity results were selected and the diameter and polydispersity index (pdi) of BBCl-lip was calculated using the Zetasizer Software (version 7.02, Malvern).

\subsection{4 $\mathrm{BBCl}$ concentration in BBCl-lip}

$\mathrm{BCl}$ concentration in BBCl-lip was determined using the UV-VIS program of a nanodrop (NanoDrop ND-100o spectrophotometer, Fisher Scientific, The Netherlands). A standard curve of 10, 25, 50, 75 and 100 $\mu \mathrm{g} / \mathrm{mL}$ of $\mathrm{BBCl}$ in water was used to determine the concentration in BBCl-lip. BBCl-lip solution was diluted $1: 10$ and $1: 20$ in methanol to dissolve the lipids. For lipid background controls, control liposomes were diluted in methanol $(1: 10,1: 20)$ and control liposomes were spiked diluted with methanol, with a final concentration of $25 \mu \mathrm{g} / \mathrm{mL}$ $\mathrm{BBCl}$. Furthermore, solvent controls included water (blank), HBS and 
methanol. Absorption was measured at $346 \mathrm{~nm}$ and the concentration of $\mathrm{BBCl}$ in $\mathrm{BBCl}-$ lip was deduced from the standard curve.

\subsubsection{Liposome composition of BBCl-lip}

The lipid composition of BBCl-lip was measured using a charged aerosol detector (CAD, Corona Ultra eso, Thermo Fisher/Dionex) coupled to an UPLC device (Ultimate 3000, Thermo Fisher/Dionex), using a BEH C18 $1.7 \mu \mathrm{m} 2.1 \times 50 \mathrm{~mm}$ column (Acquity, Waters, Ireland). A standard lipid curve containing DPPC, DSPE-PEG200o and cholesterol was prepared with the following concentrations (DPPC:DSPE-PEG2000: cholesterol in $\mathrm{mg} / \mathrm{mL}): 0.015: 0.009: 0.0045,0.150: 0.09: 0.045$, $0.375: 0.23: 0.11,0.600: 0.360: 0.180,0.750: 0.450: 0.220$ and $0.900: 0.540: 0.270$. A gradient run was used with mobile phase A $=80 \% \mathrm{MeOH}$ and $0.1 \%$ TEA in milliQ water and mobile phase $\mathrm{B}=$ $100 \% \mathrm{MeOH}$ and $0.1 \%$ TEA. Mobile phases were degassed using a water bath sonicator (Branson 2510) for $5 \mathrm{~min}$. The UPLC had a runtime of $12 \mathrm{~min} / \mathrm{sample}$ at a flow rate of $0.200 \mathrm{~mL} / \mathrm{min}$. The gradient was set as follows: $0.0-0.890 \mathrm{~min}=100 \% \mathrm{~A}, 2.0-7.56 \mathrm{~min}=100 \% \mathrm{~B}$, $7.840-12.00 \mathrm{~min}=100 \% \mathrm{~A}$. Injection volume was $4 \mu \mathrm{L}$ and column temperature was set to $50{ }^{\circ} \mathrm{C}$. Lipids were detected with peaks at $\sim 7.52$ min for DSPE-PEG200o, $~ 8.45 \mathrm{~min}$ for cholesterol and $\sim 9.1 \mathrm{~min}$ for DPPC using the CAD. Lipid concentrations in the liposomes were deduced from the standard curve. Concentrations were calculated using the accompanying software (Chromeleon Chromatography Data System version 7.1.2.1713).

\subsubsection{In vitro performance of $\mathrm{BBCl}-$ lip}

RAW 264.7 macrophages were cultured in RPMI-1640 medium substituted with 10\% FBS, $2 \mathrm{mM}$ penicillin/streptomycin and $2 \mathrm{mM} \mathrm{L}$ glutamine. Cells were split twice a week until maximum passage number 32. Cells were seeded into 96-wells cell culture plates at a concentration of $5^{*} 10^{5}$ cells $/ \mathrm{mL}$ and left to settle at $37^{\circ} \mathrm{C}$ in a humidified incubator containing $5 \% \mathrm{CO}_{2}$. After $6-8 \mathrm{~h}$, the medium was taken off and 5, 10 and $50 \mu \mathrm{M}$ of BBCl-lip or 3.9, 15.6, 62.5 and $250 \mu \mathrm{M}$ of free $\mathrm{BBCl}$ in $\mathrm{HBS}$ were added twice in triplicate per concentration to the cells and incubated for $2 \mathrm{~h}$. Medium only and control liposomes (control-lip) were used as negative control. After $2 \mathrm{~h}$, the medium was taken off and cells were incubated for $12 \mathrm{~h}$ with medium only (control) or medium containing $250 \mathrm{ng} / \mathrm{mL}$ LPS to induce an inflammatory response. After LPS stimulations, supernatant was used for cytokine expression determination using an ELISA assay. 
For cell viability measurements a $10 \%$ Alamar blue $(440 \mu \mathrm{M}$ resazurin salt in PBS) solution in medium $(v / v)$ was added to all wells and incubated for $\sim 3 \mathrm{~h}$ at $37^{\circ} \mathrm{C}$ in a humidified incubator containing $5 \% \mathrm{CO}_{2}$. Fluorescence was measured at 560/590 $\mathrm{nm}$ in a platereader (Wallac 1420 Victor3, PerkinElmer). The experiment was repeated three times $(n=3)$.

\subsubsection{IL-6 secretion determination by ELISA}

IL-6 ELISA was performed as per manufacturer's instructions with minor modifications. Briefly, immuno Maxisorp plates (Nunc art NO. 439454) were coated with coating antibody in phosphate buffered saline (PBS), sealed and left to incubate over night at room temperature (RT). Between each step, wells were washed using 0.05\% Tween 20 in PBS. After coating, cells were blocked for at least $1.5 \mathrm{~h}$ using block buffer (0.5\% BSA in PBS). First incubation: wells were either incubated with standards $(0-1000 \mathrm{pg} / \mathrm{ml})$ in $1: 2$ serial dilutions in assay diluent $(0.5 \%$ BSA and $5 \%$ FBS in PBS) in duplicates or with samples (the supernatant of the in vitro incubation assay) in triplicates for $1 \mathrm{~h}$ at RT in the dark. Second incubation: wells were incubated with detection antibody in block buffer for $1 \mathrm{~h}$ at RT in the dark. Third incubation: wells were incubated with streptavidin-HRP solution in block buffer for $1 \mathrm{~h}$ at RT in the dark. Plates were developed by adding 1-step ULTRA TMB ELISA substrate and the reaction was stopped after all concentrations of the standard showed coloration, with the same volume of Stop Solution (1.8 $\left.\mathrm{M} \mathrm{H}_{2} \mathrm{SO}_{4}\right)$. Optical density was measured at $450 \mathrm{~nm}$ using a platereader (Multiskan GO, Thermo Fisher).

\subsubsection{Animals}

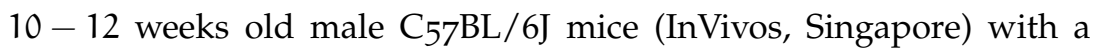
body weight between 20 and $25 \mathrm{~g}$ were used for all experiments. The mice received a standard diet and water ad libitum. All the procedures involving animal handling were performed with prior approval and in accordance with the protocols and guidelines of the Institutional Animal Care and Use Committee (IACUC) of the National University of Singapore. Per experimental group, 6-10 mice were used.

\subsubsection{Surgical procedures and cardiac function assessment}

MI was induced in mice as previously described [21]. Briefly, mice were anesthetized with a mixture of $0.5 \mathrm{mg} / \mathrm{kg}$ medetomidine, 5.0 $\mathrm{mg} / \mathrm{kg}$ Dormicum and $0.05 \mathrm{mg} / \mathrm{kg}$ fentanyl and subjected to permanent ligation of the left anterior descending artery. Mice were recovered 
by a subcutaneous injection of $0.5 \mathrm{mg} / \mathrm{kg}$ atipamezole and $5 \mathrm{mg} / \mathrm{kg}$ flumazenil followed by $0.1 \mathrm{mg} / \mathrm{kg}$ Temgesic. $100 \mu \mathrm{L} / 10 \mathrm{~g}$ body weight of control-lip, $1.5 \mathrm{mg} / \mathrm{kg} \mathrm{BBCl}$ or $1.5 \mathrm{mg} / \mathrm{kg} \mathrm{BBCl-lip} \mathrm{was} \mathrm{injected} \mathrm{via}$ the tail vein immediately after chest closure. Additional drug injections were given on day 3 and 6 after MI. The surgeon was blinded for drug delivery.

Cardiac function was assessed with a high frequency ultrasound system Vevo ${ }^{\circledR} 2100$ (Visualsonics) and analyzed with Vevo ${ }^{\circledR} 2100$ software, version 1.7.0. Echocardiography was performed on mice under general anesthesia ( $1-1.5 \%$ isoflurane) at baseline, day 7 and day 28 after MI. Body temperature was monitored with a rectal probe and maintained at $36-37{ }^{\circ} \mathrm{C}$. Volumes and functional parameters were measured in parasternal long-axis view (LV trace mode) and analyzed by a blinded researcher.

\subsubsection{Imaging of the heart}

The mice were injected with liposomes containing Cy5.5 dye (Cy5.5-lip) via the tail vain immediately after MI surgery. Hearts were harvested at day 1, 3 and 7 after surgery and snap frozen for analysis. Liposomes, accumulated in the heart were visualized with an IVIS spectrum-imaging system (PerkinElmer). The frozen left ventricles were embedded with FCS 22 Frozen Section Media (Leica Biosystems) for cryosectioning. Cryosectioning of heart tissue $(6 \mu \mathrm{m})$ were directly imaged for Cy5.5lip using a confocal microscope (FLUOVIEW FVioi Olympus) or used for histological analysis. Cryosections were stained with rat anti-mouse $\mathrm{MAC}_{3}$ monoclonal antibody (clone M\#/84, BD Biosciences) followed by horseradish peroxidase (HRP)-conjugated goat anti-rat secondary antibody (Life Technologies). Sections were developed in ImmPACT NovaRED Peroxidase (HRP) Substrate (Vector Laboratories) and counter stained with haematoxylin. The staining was analyzed with a Nikon Eclipse Ti light microscope (Nikon Instruments Inc.).

\subsubsection{Scientific calculations and statistical analysis}

$I_{50}$, maximum effect $\left(E_{\max }\right)$, and curve fit $\left(R^{2}\right)$ of IL-6 secretion inhibition by free $\mathrm{BBCl}$, were calculated in GraphPad Prism (GraphPad Prism version 5.0 for Windows) using the 'log(inhibitor) vs. response' of the 'non-linear regression of single data' analysis function. The curve was fit following the 'least squares (ordinary) fit' and the top of the curve was set to 1.0 (to which the data was normalized). Each replicate of $Y$ was considered as an individual point and the curve was fit with a maximum of 1000 iterations. 
Comparisons between the groups of mice in time were performed using a two-way ANOVA with Bonferroni post hoc analysis using SPSS software (IBM® SPSS® Statistics version 22.0). Values were reported as mean \pm SEM and a $p$-value $\leqslant 0.05$ was considered statistically significant.

\section{Conflict of Interest}

The authors declare no competing financial interest.

\section{Acknowledgment}

RAW 264.7 macrophages were a kind gift from Prof. Dr. Menno de Winther of the Department of Medical Biochemistry at the Academic Medical Center, Amsterdam, The Netherlands. This research was funded by NanoNext-NL (03D.11, RMS) and the National Medical Research Council CS-IRG grant (DPVdK) and the ATTRaCT SPF grant (DPVdK).

\section{REFERENCES}

[1] C. Allen et al. Controlling the physical behavior and biological performance of liposome formulations through use of surface grafted poly(ethylene glycol). 2002.

[2] T. M. Allen et al. "Liposomes containing synthetic lipid derivatives of poly(ethylene glycol) show prolonged circulation halflives in vivo." In: BBA - Biomembranes 1066.1 (1991), pp. 29-36.

[3] Iris E. Allijn et al. "Head-to-Head Comparison of AntiInflammatory Performance of Known Natural Products In Vitro." In: PloS one 11.5 (May 2016), e0155325.

[4] Fatih Arslan, Dominique P de Kleijn, and Gerard Pasterkamp. "Innate immune signaling in cardiac ischemia." In: Nature Reviews. Cardiology 8.5 (2011), pp. 292-300.

[5] W. Chang et al. "Berberine attenuates ischemia-reperfusion injury via regulation of adenosine- 5 '-monophosphate kinase activity in both non-ischemic and ischemic areas of the rat heart." In: Cardiovasc Drugs Ther 26.6 (2012), pp. 467-478.

[6] Jun Fang, Hideaki Nakamura, and Hiroshi Maeda. “The EPR effect: Unique features of tumor blood vessels for drug delivery, factors involved, and limitations and augmentation of the effect." In: Advanced Drug Delivery Reviews 63.3 (2011), pp. 136-151. 
[7] N. Frangogiannis. "The inflammatory response in myocardial infarction." In: Cardiovascular Research 53.1 (2002), pp. 31-47.

[8] Raju Gautam and Sanjay M. Jachak. "Recent developments in anti-inflammatory natural products." In: Medicinal research reviews 29.5 (Sept. 2009), pp. 767-820.

[9] Zhouqing Huang et al. "Berberine alleviates cardiac ischemia/reperfusion injury by inhibiting excessive autophagy in cardiomyocytes." In: European Journal of Pharmacology 762 (2015), pp. 1-10.

[10] M. Ikram. "A review on the chemical and pharmacological aspects of genus Berberis." In: Planta medica 28.4 (1975), pp. 353358.

[11] Mohsen Imanshahidi and Hossein Hosseinzadeh. "Pharmacological and therapeutic effects of Berberis vulgaris and its active constituent, berberine." In: Phytotherapy Research 22.8 (Aug. 2008), pp. 999-1012.

[12] Chi Wan Lau et al. "Cardiovascular actions of berberine." In: Cardiovascular drug reviews 19.3 (2001), pp. 234-244.

[13] Mark E. Lobatto et al. "Atherosclerotic plaque targeting mechanism of long-circulating nanoparticles established by multimodal imaging." In: ACS Nano 9.2 (2015), pp. 1837-1847.

[14] MesH Heading - Berberine. date accessed: 2016-04-11. National Institute of Health. URL: https : / /www.nlm.nih.gov/cgi/mesh / 2016/MB_cgi?mode=\&index=1947.

[15] James O. Mudd and David A. Kass. "Tackling heart failure in the twenty-first century." In: Nature 451.7181 (2008), pp. 919-928.

[16] Margherita Neri et al. "Cardiac Oxidative Stress and Inflammatory Cytokines Response after Myocardial Infarction." In: Current Vascular Pharmacology 13.1 (Mar. 2015), pp. 26-36.

[17] Gonçalo C. Pereira et al. "Mitochondrially targeted effects of berberine [Natural Yellow 18, 5,6-dihydro-9,10dimethoxybenzo(g)-1,3-benzodioxolo(5,6-a) quinolizinium] on K1735-M2 mouse melanoma cells: comparison with direct effects on isolated mitochondrial fractions." In: The Journal of pharmacology and experimental therapeutics 323.2 (Nov. 2007), pp. 636-649.

[18] Angela Pirillo and Alberico Luigi Catapano. "Berberine, a plant alkaloid with lipid- and glucose-lowering properties: From in vitro evidence to clinical studies." In: Atherosclerosis 243.2 (Dec. 2015), pp. 449-461. 
[19] R. M. Schiffelers et al. "Liposome-Encapsulated Prednisolone Phosphate Inhibits Growth of Established Tumors in Mice." In: Neoplasia 7.2 (2005), pp. 118-127.

[20] Micol Tillhon et al. "Berberine: new perspectives for old remedies." In: Biochemical pharmacology 84.10 (Nov. 2012), pp. 12601267 .

[21] Leo Timmers et al. "Toll-like receptor 4 mediates maladaptive left ventricular remodeling and impairs cardiac function after myocardial infarction." In: Circulation Research 102.2 (2008), pp. 257264 .

[22] Benjamin J. Wrigley, Gregory Y. H. Lip, and Eduard Shantsila. "The role of monocytes and inflammation in the pathophysiology of heart failure." In: European Journal of Heart Failure 13.11 (2011), pp. 1161-1171.

[23] Xiang-Hong Zeng, Xiang-Ji Zeng, and Yun-Yun Li. "Efficacy and safety of berberine for congestive heart failure secondary to ischemic or idiopathic dilated cardiomyopathy." In: The American journal of cardiology 92.2 (2003), pp. 173-176.

[24] Yao-Jun Zhang et al. "Berberine attenuates adverse left ventricular remodeling and cardiac dysfunction after acute myocardial infarction in rats: role of autophagy." In: Clinical and experimental pharmacology E physiology 41.12 (2014), pp. 995-1002. 


\title{
ENVIRONMENTAL RISK ASSESSMENT OF PHARMACEUTICALS
}

\section{Environmental impact of switching from the syn- thetic glucocorticoid prednisolone to the natural alkaloid berberine. ${ }^{12}$}

Iris E. Allijn, Rik Oldenkamp ${ }^{3}$, Gert Storm, Ad M. J. Ragas, Raymond M. Schiffelers

\begin{abstract}
Low amounts of human pharmaceuticals in the (aquatic) environment can affect bacteria, animals and ultimately humans. In this study, the environmental consequences of a shift in prescription behavior from prednisolone to berberine was modeled using an environmental decision support system based on four consecutive steps: emission, fate, exposure and effect. This model provides estimates of the relative impacts of alternative pharmaceutical prescriptions throughout Europe for both the aquatic environment and human health. Since a Defined Daily Dose (DDD) of berberine has yet to be formulated, the environmental impact of one DDD of prednisolone was compared to one DDD of berberine under the assumption of equal DDDs. Subsequently, the calculated relative impact ratio indicates how many times higher the actual DDD of berberine should be to be an environmentally better alternative for prednisolone. For the aquatic environment, this ratio exceeds 1 in all tested countries in Europe, with a median of 24.54 and a 2.5 percentile of 6.09 . This means that one DDD of berberine can be 6 times higher and still be less harmful for the aquatic environment than prednisolone. For human health, these median ratios are for conventional and advanced drinking water treatment respectively 5.87 and 22.8. However, this relative ratio does not exceed 1 in some regions in Spain, Austria, Baltic States and Finland, meaning that berberine can only be a better alternative for prednisolone as long as its DDD is lower than that of prednisolone. We conclude that for most regions in Europe
\end{abstract}

1 Manuscript in preparation

2 Part of 'Risk Analysis and Technology Assesment' (RATA) of NanoNextNL

3 Shared first author 
it is, up until a certain dose of berberine, beneficial for both the aquatic environment as well as for human health to prescribe berberine over prednisolone.

\subsection{INTRODUCTION}

Low amounts of human pharmaceuticals in the environment, even below the safety and efficacy test concentrations, can affect bacteria and animals $[7,30]$. Especially in the aquatic environment, there might be an unacceptable risk due to chronic exposure to pharmaceuticals [10]. In particular, one of the main problems caused by (human) pharmaceuticals in the environment is endocrine disruption. This includes disruption of fertility, development of feminized fish, disruption of the normal growth of fish, reptiles and aquatic invertebrates $[7,29]$. Because of their similarity in structure to endogenous hormones, endocrine disruption is often ascribed to steroidal drugs, but can be caused by nonsteroidal compounds as well [41, 17]. There is also a potential risk for humans and although the environmental concentrations are generally regarded as safe [7], it is generally unknown what fraction of pharmaceuticals eventually make their way back to humans [17]. This can be through accumulation in the food chain $[7,41,17]$ or via drinking water [30]. The presence of human pharmaceuticals in the environment appears an underestimated problem [7] with unknown consequences [17].

Already in the 1960s, the presence of human pharmaceuticals in the environment was anticipated [51] and proven in the 1980s [17]. It took until the 1990 for water contamination by pharmaceuticals to become an environmental issue [24, 53, 11]. In order to halt the environmental damage caused by human pharmaceuticals, governments may install laws to restrict the emission into the environment and provide education and return programs [13]. Despite the increased attention for the environmental impact of human pharmaceuticals since the start of the millennium [7], little regulation is currently in place [23]. The major programs that are in place, are the TSCA Chemical Substance Inventory [3] of the USA and the REACH program [2] of the EU. However, the TCSA does not involve environmental risk management for hazardous substances and the REACH does not include human pharmaceuticals [23]. Furthermore, the mandatory Environmental Risk Assessment (ERA) from the European Medicines Agency (EMA) [8], says that for all drugs the environmental impact should be assessed: the substance should be pre-screened for consumption data and for the octanol-water partitioning coefficient $\mathrm{K}_{\mathrm{ow}}$. If the $\log \mathrm{k}_{\mathrm{o} w}<4.5$, additional persistence, bioaccumulation and toxicity screenings should be 
performed. In the second phase, aquatic toxicology, emission and fate should be assessed in a refined risk assessment. However, from these tests the vitamins, electrolytes, amino acids, peptides, proteins, carbohydrates, lipids, vaccines and natural products are exempted. Furthermore, tests results cannot be a criterion for refusal for marketing [8].

To be able to predict the environmental consequences of specific molecules, it is important to have proper fate and effect assessment protocols in operation. These protocols can help to predict whether a compound can reach hazardous concentrations in the aquatic, terrestrial or atmospheric environment $[17,43,42]$. This would complement the safety-testing of pharmaceutical companies since these tests are of limited duration in mice and rats and therefore do not mimic the environmental conditions. Furthermore, no tests are performed on algae, fishes and water fleas [3o]. Up to now, pharmaceutical companies have only to a limited extent included environmental impact in their selection of active pharmaceutical ingredients. Including this element in the development and selection of new active pharmaceutical ingredients is advocated to make the transition to green pharmacy and chemistry [13, $17,40,33]$.

Corticosteroids are the most potent anti-inflammatory agents and are both prescribed as over-the-counter drugs in certain countries as well as prescription-only medication in others. They are extensively used for a variety of conditions. The prescribed dose and administration route depends on the severity of the inflammatory symptoms and medical condition [25, 59]. Prednisolone (Figure 7.1) is a wellknown synthetic corticosteroid, mainly prescribed in inflammatory diseases such as rheumatoid arthritis. Available metabolism data of prednisolone show that up to $24 \%$ is excreted in its unchanged form [6]. Furthermore, prednisolone is the active form of prednisone, which is an often used pharmaceutical as well. Altogether, this implies that a substantial amount of prednisolone ends up in the environment [6]. Synthetic steroids are designed to have strongly enhanced potencies compared to natural hormones. As a result, they are likely to induce endocrine disruption in aquatic organisms. But also immune depression and neurobehavioral changes have been reported [18, 27]. Their synthetic nature may makes them less biodegradable, increasing environmental concentrations [48].

Natural products are gaining interest because of their potent antiinflam-matory and anti-oxidant properties [22, 21, 52]. The therapeutic window of natural products might be larger than for corticosteroids. A promising anti-inflammatory natural product that is currently under investigation is the isoquinoline quarternary alkaloid berberine [34] (Figure 7.1). It has been shown to reduce inflammation in vitro via different pathways than prednisolone [5]. However, the impact of berberine on the environment has not yet been investigated. 
In this study, we aimed to model the environmental consequences of a shift in prescription behavior from the synthetic corticosteroid prednisolone to the natural product berberine. We hypothesized that berberine due to its natural source would have less environmental impact than the synthetic drug prednisolone. Human and aquatic toxicity and fate parameters of the compounds were fed into a model as previously described by Oldenkamp et al. [43] and used to assess the environmental impact of pharmaceutical prescriptions throughout Europe. This model was used to describe the relative impact of berberine and prednisolone on the environment and quantifies the health benefits for the general population to the risks caused by environmental exposure.

\subsection{METHODOLOGY}

\subsubsection{Description of the model}

The environmental decision support system described by Oldenkamp et al. [42] was used to model the environmental consequences of a shift in prescription behavior from prednisolone to berberine. This methodology was originally developed for the location specific assessment and comparison of the environmental impact of two alternative pharmaceutical prescriptions, aiming to provide physicians with the opportunity to include environmental considerations in their prescription practice. The model provides regionalized estimates of the relative impacts of alternative pharmaceutical prescriptions throughout Europe, for both the aquatic environment and human health. It is based on the four consecutive steps of emission, fate, exposure and effect estimation (Figure 7.2).

As a first step, emissions into wastewater are calculated as the sum of non-compliance and subsequent disposal via flushing, and of actual consumption and excretion as parent compound via urine or feces. Pharmaceutical residues in wastewater are estimated at the level of
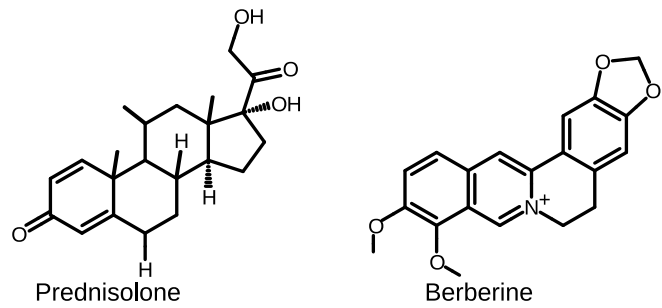

Figure 7.1: Molecular structures of prednisolone (CAS RN $=\mathbf{5 0 - 2 4 - 8}$ ) and berberine (CAS RN = 2086-83-1). 
individual Member States, before they are divided over individual agglomerations, based on their population size. Generally, this wastewater is discharged into the surface water after passage through a sewage treatment plant (STP). However, part of the wastewater might also be discharged into the surface water directly, depending on the local level of STP-connectivity. Indirect emissions (i.e. after passage through an STP) are calculated at the level of the individual STPs, and depend on STP design and active pharmaceutical ingredient (API)-specific removal rates corresponding with the treatment techniques applied. Furthermore, the model also estimates emissions of APIs to agricultural soils, which depend on pharmaceutical levels in secondary sewage sludge and Member State specific sludge disposal practices.

To enable multimedia fate calculations with the model SimpleBox (e.g. Hollander et al., 2009 [26]), emissions to surface water and agricultural soils are aggregated at the level of $100^{*} 100 \mathrm{~km}$ environmental grid cells, spatially parameterized with data from Pistocchi et al. [45]. These calculations result in yearly averages steady-state surface water and soil concentrations. Relative aquatic risk quotients are calculated as the ratio between these concentrations and API-specific $\mathrm{HC}_{50}$ values (i.e. the concentration at which $50 \%$ of the individuals in $50 \%$ of the aquatic species is being affected). Finally, these risk quotients are used to derive a grid-specific prioritization of the APIs assessed, based on their relative aquatic impacts.

The calculation of relative human health risk quotients requires additional exposure calculations, including estimations of the transfer of pharmaceutical residues into foodstuffs and drinking water, and age- and location-specific behavioral and consumption patterns. Human contact media taken into account are drinking water, fruits and vegetables, meat products, milk products, fish, surface water, and soil. The degree of exposure is determined by the concentrations in these contact media as well as the intensity of the contact with them. Concentrations in food were estimated from those in surface water and agricultural pore water using bioconcentration factors (BCFs) for fish [20], root concentration factors (RCFs) for fruits and vegetables [55] and biotransfer factors (BTFs) for meat and milk products [56].

Concentrations in drinking water depend on the source of the water and the purification techniques applied. While data on the first were available at the level of the individual EU Member States [15], spatially explicit data on drinking water purification levels were not. The model addresses this by formulating different purification scenarios: conventional, advanced and no treatment. The conventional purification scenario was regarded as the minimum scenario in order for the EU Member States to meet the European quality standards (EU Council Directive $98 / 83 / E C)$, and it consists of in series application of coagulation, powdered activated carbon (PAC), chlorination and sand filtration. In 
addition to these techniques, the advanced treatment scenario also includes the application of membrane bioreactor (MBR), UV-treatment, ozonation, reverse osmosis and nanofiltration. The scenario of no treatment was specifically formulated to address small scale groundwater sources and was excluded from the present study.

Then, the model derives average daily human exposure estimations for a range of exposure groups, characterized by age, nationality and other factors (e.g. drinking water purification level and food origin). In a previous study, infants ( $0-1$ years) that consumed locally produced foodstuffs were identified as the most sensitive human exposure group [43]. Therefore, we selected them as the human exposure group most suitable for the calculation of the impact on human health. Human health risk quotients were calculated similar to those for the aquatic environment: as the ratio between the average daily exposure and APIspecific $\mathrm{HD}_{50}$ values (i.e. the dose at which $50 \%$ of the individuals in $50 \%$ of the mammalian species is being affected).

The environmental decision support system described by Oldenkamp et al. [42] compares two alternative pharmaceutical prescriptions on the basis of their Defined Daily Doses (DDDs). The study presented here, however, aims to estimate the environmental consequences of a shift in prescription behavior from prednisolone to berberine. However, DDDs have yet to be formulated for berberine. Therefore, the environmental impact of one DDD of prednisolone is compared with one DDD of berberine, assuming that their DDDs would be equal. This is done for each environmental grid cell throughout Europe. The ratio between the impacts of the two prescriptions then indicates how many times higher the actual DDD of berberine could be for it to form an environmentally better alternative for prednisolone.

\subsubsection{Parameterization of the model}

Substance-specific parameters were consistently parameterized according to a four-step preference approach:

1. Experimental or measurement data

2. Extrapolation from related data (e.g. from degradation rates in other environmental media)

3. Structure or property based predictions (e.g. the use of quantitative structure activity relationships (QSARs))

4. Worst-case assumptions

This enables an interpretation of the results through an analysis of the input data for prednisolone and berberine (Table 7.1). When important data gaps exist for prednisolone (i.e. worst-case assumptions are 


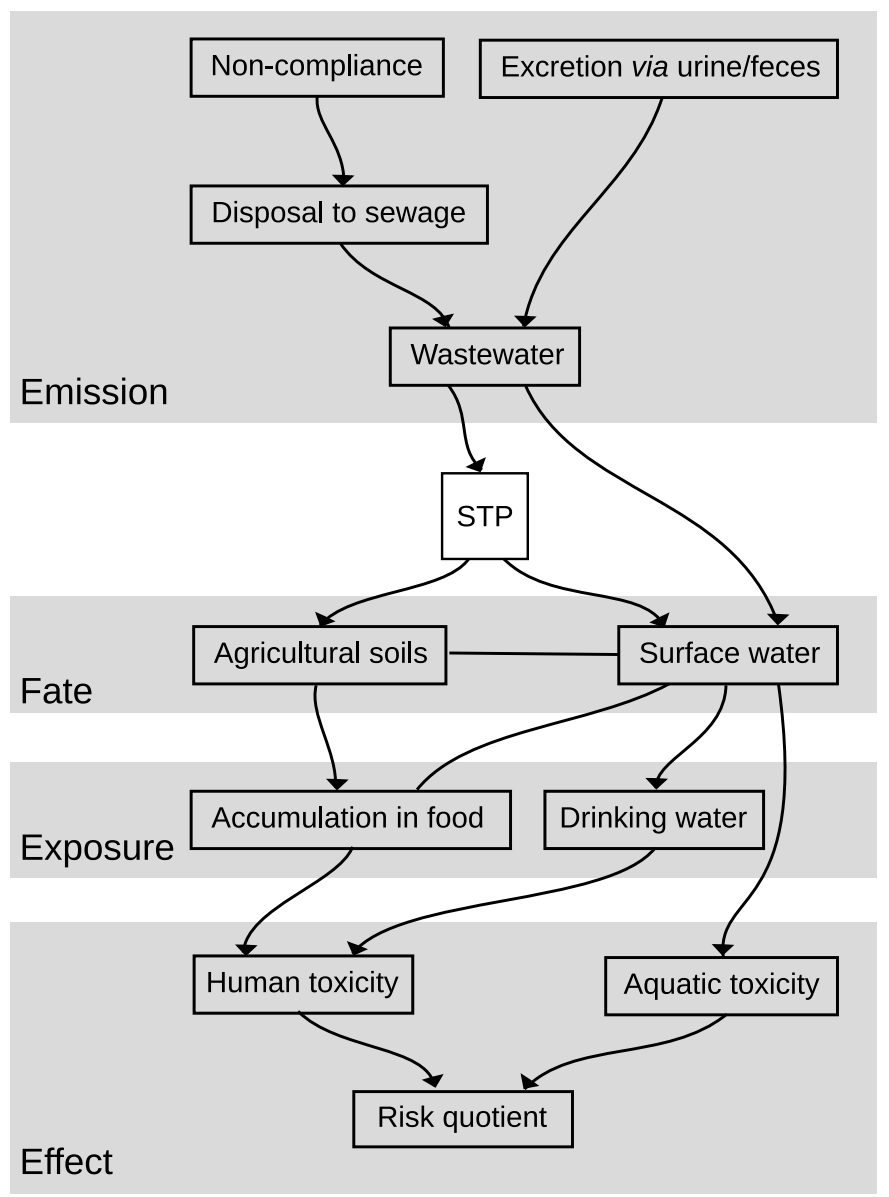

Figure 7.2: Schematic visualization of the processes used for the calculations. Adjusted from Figure Ai from Oldenkamp et al., 2014 [42]. 
used), its environmental impact might be overestimated and the suitability of berberine as environmentally better alternative might then also be overestimated. Similarly, when important data gaps exist for berberine, the model might underestimate its suitability as environmentally better alternative for prednisolone.

Table 7.1: API-specific input parameters. STP = sewage treatment plant, ${ }^{a}$ Calculated as the fraction removed during combined UV- and $\mathrm{O}_{3}$ treatment, minus the fraction removed after $\mathrm{O}_{3}$ alone.

\begin{tabular}{|c|c|c|}
\hline Parameter & BER (Pref\#) & PRED (Pref\#) \\
\hline Excretion as parent compound (-) & $0.00014(1)[44]$ & $0.24(1)[6]$ \\
\hline Vapor pressure $(\mathrm{Pa})$ & $1.4^{*} 10^{-7}(3)[14]$ & $1.6^{*} 10^{-12}(3)[50]$ \\
\hline Water solubility $\left(\mathrm{mg}^{*} \mathrm{~L}^{-1}\right)$ & $0.354(3)[57]$ & $223(3)[37]$ \\
\hline $\log k_{o w}(-)$ & $2.1(3)[9]$ & $1.62(1)[37]$ \\
\hline$k_{b i o, S T P}\left(h^{-1}\right)$ & $0(4)$ & $0.0070(1)[16]$ \\
\hline STP N-removal (-) & $0(4)$ & O (4) \\
\hline STP P-removal (-) & $0(4)$ & O (4) \\
\hline STP UV-treatment removal (-) & $0.32^{a}(1)[46]$ & $0(4)$ \\
\hline STP ozonation removal (-) & $0.50(1)[46]$ & $0(4)$ \\
\hline STP chlorination removal (-) & $0(4)$ & $0(4)$ \\
\hline STP sand filtration removal (-) & $0(4)$ & $0(4)$ \\
\hline STP microfiltration removal (-) & $0(4)$ & O (4) \\
\hline$k_{\text {bio, water }}\left(\mathrm{S}^{-1}\right)$ & O (4) & $0(4)$ \\
\hline kphoto,water $\left(S^{-1}\right)$ & $O(4)$ & $4.15^{*} 10^{-5}(1)[12]$ \\
\hline$k_{\text {hydro,water }}\left(\mathrm{S}^{-1}\right)$ & O (3) [35] & $0(4)$ \\
\hline$k_{\text {deg,soil }}\left(S^{-1}\right)$ & $O(4)$ & $O(4)$ \\
\hline$k_{d e g, s e d}\left(S^{-1}\right)$ & O (4) & $0(4)$ \\
\hline DWT conventional removal (-) & $0.35(1 / 4)[47]$ & $0(4)$ \\
\hline DWT advanced removal (-) & $0.93(1 / 4)[47,58]$ & $0(4)$ \\
\hline Aquatic $\mathrm{HC}_{50}\left(\mathrm{mg}^{*} \mathrm{~L}^{-1}\right)$ & 14.26 (1) $[4,19,32,31,38,39,49]$ & 2.26 (1) [12] \\
\hline Mammalian $\mathrm{HD}_{50}\left(\mathrm{mg}^{*} \mathrm{kgbw}^{-1 *} \mathrm{~d}^{-1}\right)$ & $763.50(1)[60,28]$ & 496.95 (1) $[1,36,54]$ \\
\hline
\end{tabular}




\section{$7 \cdot 3$ RESULTS}

\subsubsection{Aquatic environment}

The ratios between the aquatic risk quotients for prednisolone and berberine are given in Figure 7·3. A value of 1 indicates that, at equal dose, a prescription of berberine and a prescription of prednisolone have equal impacts on the aquatic environment. A value $<1$ indicates that berberine might only be an environmentally beneficial alternative for prednisolone if its prescribed dose were lower. A value $>1$ indicates that berberine could still be considered an environmentally beneficial alternative for prednisolone, even if it were prescribed at a higher dose. In all grid cells throughout Europe, the ratio between relative aquatic risk quotients for prednisolone and berberine exceeds 1 , with a median of 24.54 and $2.5^{\text {th }}$ percentile of 6.09 (Table 7.2). This means that as long as the equivalent therapeutic dose of berberine is approximately 6 times that of prednisolone or less regardless of location, this compound has less damaging impact on the aquatic environment and is the environmentally prefered choice for prescription. However, if the specific prescription location is known, the dosage of berberine might be up until 24 times that of prednisolone.

\subsubsection{Human health}

Similar to the aquatic environment, the health impact on infants after consumption of water after conventional DWT is given as the ratio of relative risk quotient for prednisolone and berberine (Figure 7.4). In most tested countries in Europe, berberine has a lower impact on infants' health than prednisolone with a median ratio 5.87 (Table 7.2). This means that the therapeutic dose of berberine can be almost 6 times higher than prednisolone and still be beneficial. However, the $2.5^{\text {th }}$ percentile of the ratios throughout Europe is below 1 (0.61), meaning that for at least 1 in 40 grid cells, berberine would only be environmentally beneficial with a prescribed dose below that of prednisolone. This holds for some regions in Spain, Austria, Baltic States and Finland. Prescription of berberine over prednisolone is most beneficial in Sweden and The Netherlands.

The impact on infants after consumption of water after advanced DWT is again in favor of berberine over prednisolone in most tested countries in Europe (Figure 7.5). In the countries where some regions only were beneficial for prednisolone, the equilibrium has shifted more towards the favor of berberine. Advanced water treatment enhanced the favoring position of berberine over prednisolone in all countries except for Austria. Again, Sweden and The Netherlands stand out. The 


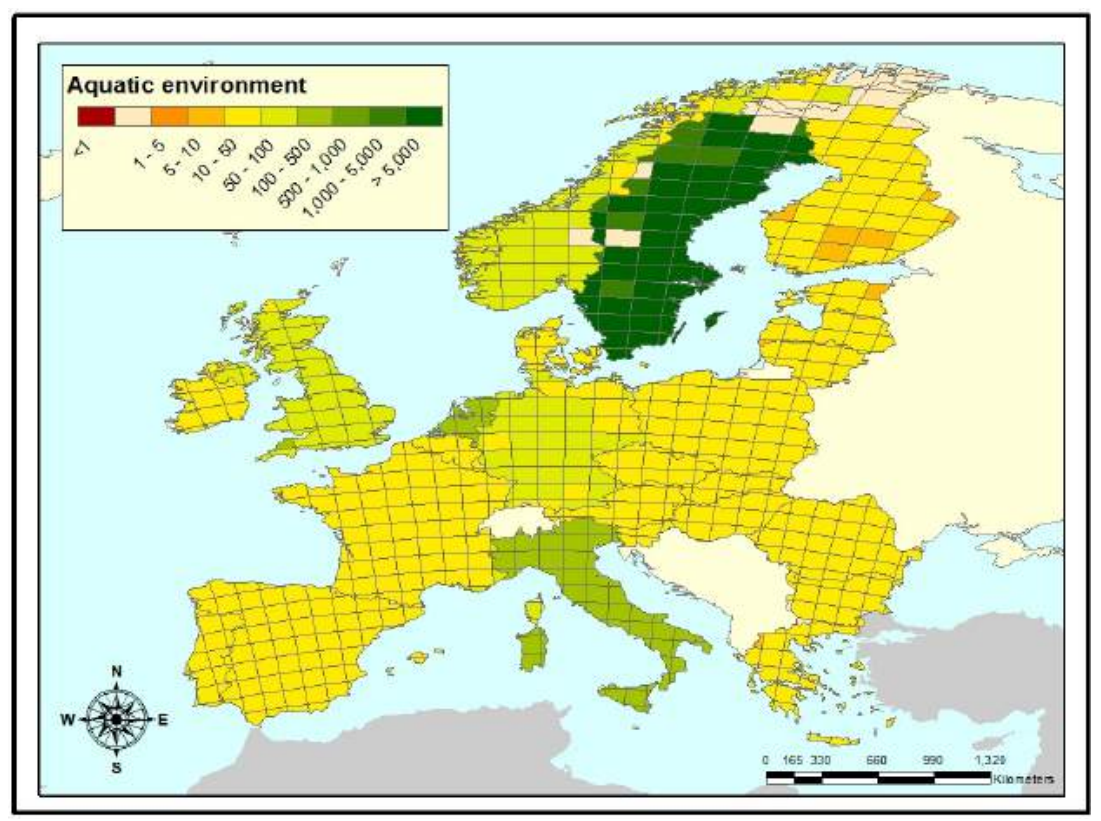

Figure 7.3: Ratios between the relative aquatic risk quotients for prednisolone and berberine. In white grid cells, the region is sparsely populated without emission or there are no data available on the STPs. In all grid cells prednisolone/berberine is larger than 1, which means that it is beneficial to prescribe berberine over prednisolone up until a certain dosage. 
therapeutic dose of berberine can now be 22.8 (median) times higher than prednisolone (Table 7.2).

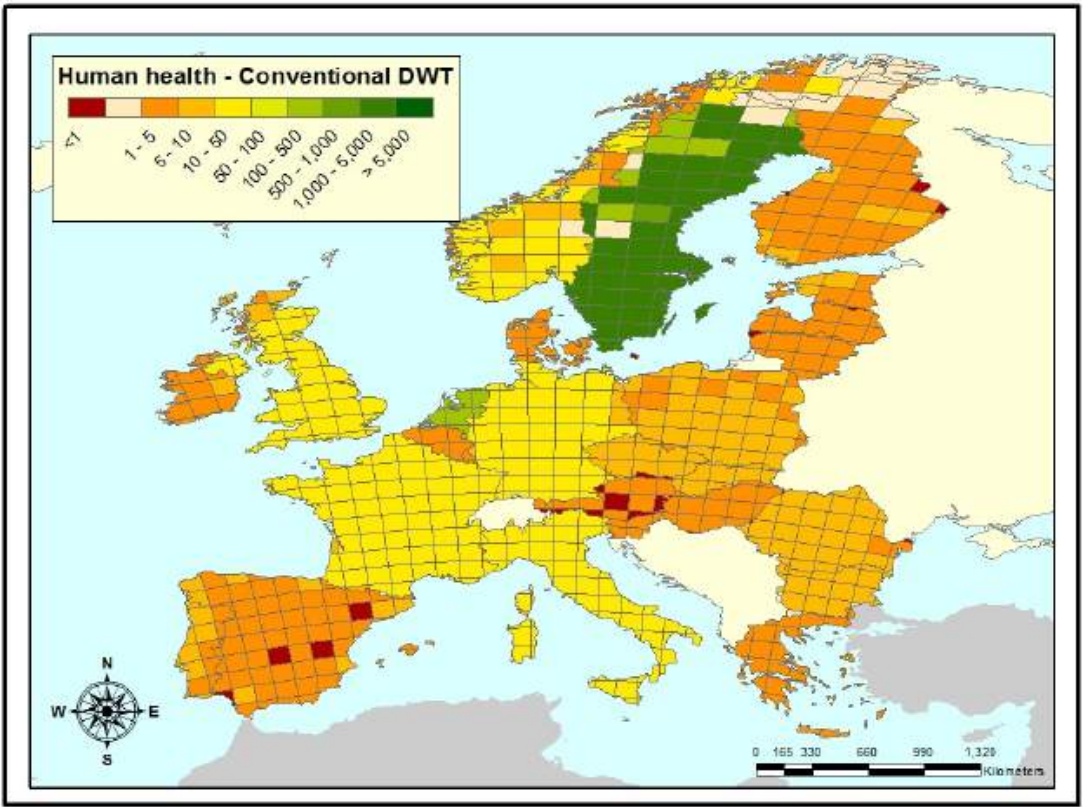

Figure 7.4: Ratios of human health risk quotients for prednisolone and berberine after conventional drinking water treatment. In white grid cells, the region is sparsely populated without emission or there are no data available on the STPs. When prednisolone/berberine is smaller than 1 (red grids), this means that for berberine to form an environmentally beneficial alternative for prednisolone, its therapeutic dose should be lower. In all grid cells with a value larger than 1 the therapeutic dose of berberine might exceed that of prednisolone up until a certain dose, and still be an environmentally beneficial alternative.

Table 7.2: Ratios of risk quotients for prednisolone and berberine for the aquatic environment and for infants after consumption of conventional or advanced DWT. Sparsely populated grids without emissions are not taken into account in these calculations. DWT $=$ drinking water treatment.

\begin{tabular}{llll}
\hline & Aquatic environment & Infants - conventional DWT & Infants - advanced DWT \\
\hline Average & 663.27 & 174.73 & 775.56 \\
$2.5^{\text {th }}$ percentile & 6.09 & 0.62 & 0.62 \\
Median & 24.54 & 5.87 & 22.80 \\
\hline
\end{tabular}




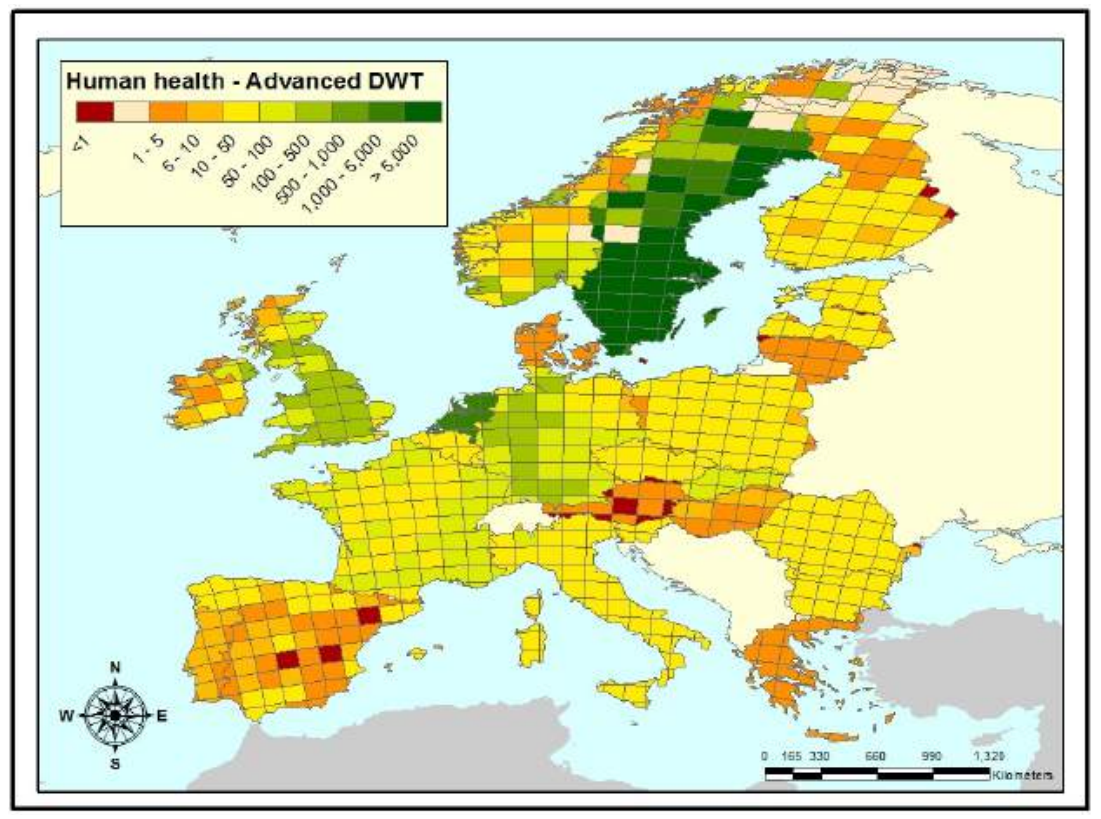

Figure 7.5: Ratios of human health risk quotients for prednisolone and berberine after advanced drinking water treatment. In white grid cells, the region is sparsely populated without emission or there are no data available on the STPs. When prednisolone/berberine is smaller than 1 (red grids), this means that for berberine to form an environmentally beneficial alternative for prednisolone, its therapeutic dose should be lower. In all grid cells with a value larger than 1 , the therapeutic dose of berberine might exceed that of prednisolone up until a certain dosage and still be an environmentally beneficial alternative. 


\subsection{DISCUSSION}

Although several chemical substance regulation programs are in place (TSCA Chemical Substance Inventory in the USA, REACH and ERA in Europe), these programs are incomplete when it comes to protection of the (aquatic) environment and human health due to exposure to this environment [23]. It is important to look beyond the impact of pharmaceuticals on individual patients and include the environmental impact as well. Therefore, in this study, we modeled the environmental consequences of a shift in prescription behavior from the synthetic corticosteroid prednisolone to the natural product berberine. An environmental decision support system [42] was used to map the results.

In order to predict the environmental impact of prednisolone and berberine, this model was fed with API-specific parameters (Table 7.1). To get to the most realistic results, experimental and calculated data should be used to parameterize the model. However, in the case of both prednisolone and berberine, only few data was available and worst case assumptions (\#4 of the parameterization of the model) had to be made to model the fate of these APIs during STP and DWT processes.

Since the actual therapeutic dose of berberine is unknown, it is not possible to say that the environmental impact of berberine over prednisolone is per se less severe as long as the relative impact ratio $>1$. However, we can argue that as long as the ratio of the therapeutic doses of prednisolone/berberine does not exceed the ratios of their environmental impacts, that berberine is indeed the environmentally friendly alternative. Similarly however, berberine could still be the environmentally friendly alternative if the impact ratio $<1$, taken that the therapeutic dose of berberine should then be lower than that of prednisolone.

We showed that in all tested grid cells in Europe, the aquatic impact ratios of prednisolone/berberine $>1$, with a median of 24.54 (Figure $7 \cdot 3$, Table 7.2). This means that as long as the therapeutic dose of berberine $<6$ times the dose of prednisolone, it is indeed less damaging for the aquatic environment in $97.5 \%$ of the EU regions modelled.

The impact on human health in infants after consumption of water after (conventional and advanced) DWT is except for some regions in Spain, Austria, The Baltic States and Finland a relative impact ratio $>1$, meaning that up to a defined dose (respectively 5.5 and 22 times the dose of prednisolone), indeed berberine is the environmentally friendly alternative (Figures $7 \cdot 4$ and 7.5, Table 7.2).

In all three tested conditions, Sweden and The Netherlands stand out, and can tolerate higher doses of berberine in order to be still beneficial over prednisolone. This is due to the fact that in these countries less pharmaceuticals are directly flushed through the toilet. Normally, 
this route of emission does not have a great impact on the environmental outcome. However, after consumption only $0.01 \%$ of berberine against $24 \%$ of prednisolone is excreted from the body, and thus in this case, it makes a clear difference.

\subsection{CONCLUSION}

This study was performed to raise awareness in the pharmaceutical community on the impact of pharmaceuticals on the (aquatic) environment and that simple changing from one drug to another could be beneficial for both aquatic as human health. As an example, the synthetic corticosteroid prednisolone was compared to the natural alkaloid berberine. We showed that in most regions in Europe, it is up until a certain dose of berberine, beneficial for both the aquatic environment as well as for human health to prescribe berberine over prednisolone. Additional regulations on the use of pharmaceuticals, taking prescription of alternative drugs into account, would be a good start towards a safer (aquatic) environment.

\section{Conflict of Interest}

The authors declare no competing financial interest.

\section{Acknowledgment}

This work was financially supported by NanoNextNL grant 03D.11 (R. M. Schiffelers) and

\section{REFERENCES}

[1] Mohamed M. Abdulla. "Anti-inflammatory activity of heterocyclic systems using abietic acid as starting material." In: Monatshefte für Chemie - Chemical Monthly 139.6 (June 2008), pp. 697705 .

[2] European Chemical Agency. Registration, Evaluation, Authorisation and Restriction of Chemicals. URL: http : / / echa . europa. eu / regulations/reach (visited on 04/20/2016).

[3] United States Environmental Protection Agency. TSCA Chemical Substance Inventory. (Visited on 04/20/2016). 
[4] Shaukat Ali, Danielle L. Champagne, and Michael K. Richardson. "Behavioral profiling of zebrafish embryos exposed to a panel of 6o water-soluble compounds." In: Behavioural brain research 228.2 (Mar. 2012), pp. 272-283.

[5] Iris E. Allijn et al. "Head-to-Head Comparison of AntiInflammatory Performance of Known Natural Products In Vitro." In: PloS one 11.5 (May 2016), eo155325.

[6] Jean-Philippe Besse, Christine Kausch-Barreto, and Jeanne Garric. "Exposure Assessment of Pharmaceuticals and Their Metabolites in the Aquatic Environment: Application to the French Situation and Preliminary Prioritization." In: Human and Ecological Risk Assessment: An International Journal 14.4 (July 2008), pp. 665695.

[7] Alistair B. A. Boxall. "The environmental side effects of medication." In: EMBO reports 5.12 (2004), pp. 1110-1116.

[8] CHMP. Guideline on the environmental risk assessment of medicinal products for human use. Tech. rep. 2006. URL: http : / / www . ema . europa . eu / docs / en\%7B\%5C_\%7DGB / document $\% 7 B_{-} \% 7 \mathrm{Dlibrary} /$ 0ther/2011/04/WC500105107. pdf.

[9] “Clarke's Analysis of Drugs and Poisons: Berberine." In: Fourth edition. 2011.

[10] Mark Crane, Chris Watts, and Tatiana Boucard. "Chronic aquatic environmental risks from exposure to human pharmaceuticals." In: Science of the Total Environment 367.1 (2006), pp. 23-41.

[11] C. G. Daughton and T. A. Ternes. "Pharmaceuticals and personal care products in the environment: agents of subtle change?" In: Environmental health perspectives 107 Suppl (Dec. 1999), pp. 907938.

[12] Marina DellaGreca et al. "Toxicity of prednisolone, dexamethasone and their photochemical derivatives on aquatic organisms." In: Chemosphere 54.5 (Feb. 2004), pp. 629-637.

[13] Nora A. Doerr-MacEwen and Murray E. Haight. "Expert stakeholders' views on the management of human pharmaceuticals in the environment." In: Environmental management 38.5 (Nov. 2006), pp. 853-866.

[14] US EPA. Estimation Program Interface Suite for Microsoft Windows. 2012.

[15] Eurostat. Annual freshwater abstraction by source and sector. 2016. URL: http://ec . europa. eu/eurostat/data/database. 
[16] Zhanlan Fan et al. "Behaviors of glucocorticoids, androgens and progestogens in a municipal sewage treatment plant: comparison to estrogens." In: Environmental science E technology 45.7 (Apr. 2011), pp. 2725-2733.

[17] Despo Fatta-Kassinos, Sureyya Meric, and Anastasia Nikolaou. "Pharmaceutical residues in environmental waters and wastewater: Current state of knowledge and future research." In: Analytical and Bioanalytical Chemistry 399.1 (2011), pp. 251-275.

[18] Karl Fent, Anna a Weston, and Daniel Caminada. "Ecotoxicology of human pharmaceuticals." In: Aquatic toxicology 76.2 (Feb. 2006), pp. 122-159.

[19] F. Freiburghaus et al. "Evaluation of African medicinal plants for their in vitro trypanocidal activity." In: 66 55.1 (Dec. 1996), pp. 111.

[20] Wenjing Fu, Antonio Franco, and Stefan Trapp. "Methods for estimating the bioconcentration factor of ionizable organic chemicals." In: Environmental Toxicology and Chemistry 28.7 (2009), p. 1372.

[21] María Galvez-Llompart, Riccardo Zanni, and Ramón GarcíaDomenech. "Modeling natural anti-inflammatory compounds by molecular topology." In: International journal of molecular sciences 12.12 (Jan. 2011), pp. 9481-9503.

[22] Raju Gautam and Sanjay M. Jachak. "Recent developments in anti-inflammatory natural products." In: Medicinal research reviews 29.5 (Sept. 2009), pp. 767-820.

[23] Melanie Gross et al. "Environmental risk assessment of human pharmaceuticals: Regulatory developments." In: Integrated Environmental Assessment and Management February 2015 (2015).

[24] B. Halling-Sorensen et al. "Occurence, fate and effects of pharmaceuticals substance in the environment - A review." In: Chemosphere 36.2 (1998), pp. 357-393.

[25] Stephen G. Hillier. "Diamonds are forever: the cortisone legacy." In: The Journal of endocrinology 195.1 (Oct. 2007), pp. 1-6.

[26] Anne Hollander et al. In: Environmental toxicology and chemistry 1 ().

[27] Keisuke Ikehata, Naeimeh Jodeiri Naghashkar, and Mohamed Gamal El-Din. "Degradation of Aqueous Pharmaceuticals by Ozonation and Advanced Oxidation Processes: A Review." In: Ozone: Science E Engineering 28.6 (2006), pp. 353-414.

[28] G. D. Jahnke and C. J. Price. "Developmental toxicity evaluation of berberine in rats and mice." In: Birth Defects Research 77.3 (June 2006), pp. 195-206. 
[29] Susan Jobling et al. "Widespread Sexual Disruption in Wild Fish." In: Environmental Science \& Technology 32.17 (Sept. 1998), pp. 2498-2506.

[30] Sushil K. Khetan and Terrence J. Collins. "Human pharmaceuticals in the aquatic environment: a challenge to Green Chemistry." In: Chemical reviews 107.6 (June 2007), pp. 2319-2364.

[31] Sung Han Kim et al. "Antimicrobial activity of 9-O-acyl- and 9-Oalkylberberrubine derivatives." In: Planta medica 68.3 (Mar. 2002), pp. 277-281.

[32] Y. C. Kim et al. "Biologically active quinolone alkaloids from Evodia rutaecarpa on Artemia salina." In: Planta medica 64.5 (June 1998), p. 490.

[33] Klaus Kümmerer. "The presence of pharmaceuticals in the environment due to human use-present knowledge and future challenges." In: Journal of environmental management 90.8 (June 2009), pp. 2354-2366.

[34] Zheng Li et al. "Antioxidant and Anti-Inflammatory Activities of Berberine in the Treatment of Diabetes Mellitus." In: Evidence-based complementary and alternative medicine : eCAM 2014 (Jan. 2014), p. ID 289264.

[35] W. J. Lyman, W. F. Reehl, and D. H. Rosenblatt. Handbook of Chemical Property Estimation Methods. 1990, pp. 7-4, 7-5, 8-12.

[36] E. Mascitelli-Coriandoli and A. Fraia. "Prednacinolone, a new anti-inflammatory steroid. 2. Biochemical and pharmacological properties." In: Arzneimittel-Forschung 20.1 (Jan. 1970), pp. 111115.

[37] National Library of Medicine (US). ChemIDplus a TOXNET Database: Prednisolone. 2016. URL: http : //chem.sis.nlm.nih.gov/chemidplus/rn/50-24-8.

[38] E. Mongelli et al. "Cytotoxic 4-nerolidylcatechol from Pothomorphe peltata inhibits topoisomerase I activity." In: Planta medica 4 (), pp. 376-378.

[39] D. Morrow, D. Corrigan, and S. Waldren. In: Planta medica 9 ().

[40] Margie Nearing. "The green pharmacy. Herbal medicines in modern usage." In: The IDRC reports 14.1 (1985), pp. 10-11.

[41] Anastasia Nikolaou, Sureyya Meric, and Despo Fatta. "Occurrence patterns of pharmaceuticals in water and wastewater environments." In: Analytical and Bioanalytical Chemistry 387.4 (2007), pp. 1225-1234. 
[42] Rik Oldenkamp et al. "Environmental impact assessment of pharmaceutical prescriptions: Does location matter?" In: Chemosphere 115 (Nov. 2014), pp. 88-94.

[43] Rik Oldenkamp et al. "Spatially explicit prioritization of human antibiotics and antineoplastics in Europe." In: Environment international 51 (Jan. 2013), pp. 13-26.

[44] J. F. Pan et al. "Identification of three sulfate-conjugated metabolites of berberine chloride in healthy volunteers' urine after oral administration." In: Acta pharmacologica Sinica 23.1 (Jan. 2002), pp. $77-82$.

[45] A. Pistocchi and D. Pennington. "European hydraulic geometries for continental SCALE environmental modelling." In: Journal of Hydrology 329.3-4 (Oct. 2006), pp. 553-567.

[46] Weiwei Qin et al. "Treatment of berberine hydrochloride pharmaceutical wastewater by $\mathrm{O}_{3} / \mathrm{UV} / \mathrm{H}_{2} \mathrm{O}_{2}$ advanced oxidation process." In: Environmental Earth Sciences 73.9 (May 2015), pp. 49394946.

[47] Guanglei Qiu et al. "Combination of upflow anaerobic sludge blanket (UASB) and membrane bioreactor (MBR) for berberine reduction from wastewater and the effects of berberine on bacterial community dynamics." In: Journal of hazardous materials 246247 (Feb. 2013), pp. 34-43.

[48] Shen Qu et al. "Product-to-parent reversion of trenbolone: unrecognized risks for endocrine disruption." In: Science 342.6156 (Oct. 2013), pp. 347-351.

[49] S. Saeidnia et al. "Biological activity of two red algae, Gracilaria salicornia and Hypnea flagelliformis from Persian Gulf." In: Pharmacognosy Research 1.6 (2009), pp. 428-430.

[50] Chemical Abstract Service. SciFinder. 2013. URL: https : //scifinder. cas.org.

[51] Elisabeth Stumm-Zollinger and Gordon M. Fair. "Biodegradation of Steroid Hormones." In: Water Pollution Control Federation 37.11 (1965), pp. 1506-1510.

[52] Christy C. Tangney and Heather E. Rasmussen. "Polyphenols, inflammation, and cardiovascular disease." In: Current atherosclerosis reports 15.5 (May 2013), p. 324.

[53] Thomas A. Ternes. "Occurrence of drugs in German sewage treatment plants and riversiDedicated to Professor Dr. Klaus Haberer on the occasion of his 7oth birthday.1." In: Water Research 32.11 (1998), pp. 3245-3260.

[54] George Tonelli. "Acute toxicity of corticosteroids in the rat." In: Toxicology and Applied Pharmacology 8.2 (Mar. 1966), pp. 250-258. 
[55] Stefan Trapp. "Modelling uptake into roots and subsequent translocation of neutral and ionisable organic compounds." In: Pest Management Science 56.9 (2000), pp. 767-778.

[56] C. C. Travis and Ad Arms. "Bioconcentration of organics in beef, milk, and vegetation." In: Environmental science E technology 22.3 (1988), pp. 271-274.

[57] VCCLAB. Virtual Computational Chemistry Laboratory. 2005. URL: http: //www. vcclab.org.

[58] Shu $\mathrm{Hu}$ Xiao et al. "Comparative Study on the Treatment of Berberine Wastewater by Chemical- and Electro-Coagulation Processes: Zeta Potential Analysis." In: Advanced Materials Research 599 (Nov. 2012), pp. 496-500.

[59] Nan Yang, David W Ray, and Laura C Matthews. "Current concepts in glucocorticoid resistance." In: Steroids 77.11 (Sept. 2012), pp. 1041-1049.

[6o] Xiaoli Ye et al. "Synthesis and antihyperlipidemic efficiency of berberine-based HMG-CoA reductase inhibitor." In: Medicinal Chemistry Research 21.7 (May 2011), pp. 1353-1362. 



\section{SUMMARIZING \\ DISCUSSION}

\section{NATURAL PRODUCTS TO TARGET INFLAMMATION}

Most modern, or Western lifestyle, diseases such as type 2 diabetes mellitus (T2DM), cardiovascular disease (CVD) and cancer have a chronic inflammatory process at its base. Much is known about acute inflammation, which is beneficial to respond to injury. However, less is known on the pathways that, when the acute phase is unresolved, drive the transition to chronic inflammation (Chapter 1). Acute inflammation, the transition to chronic inflammation and the onset of these diseases all can be linked to metabolic syndrome (MetSyn) (Figure 1.1).

To be able to reduce patient suffering and diminish the economic and social burden these diseases pose, it is important to explore new possible treatments. The fact that these diseases appear interlinked through chronic inflammation provides attractive pathways to modulate a range of diseases. Although the reduction of chronic inflammation may not be curative, it can act synergistically with other treatment modalities. Of course, currently many potent anti-inflammatory drugs have been identified. However, these potent established drugs like prednisolone, have a substantial set of adversary effects associated with chronic use. For corticosteroids, this may eventually even lead to Cushing's Syndrome. This condition, as it turns out, is well connected with MetSyn and hence prescription of glucocorticoids could maintain the vicious circle of chronic inflammation. It is, therefore, of great importance to find alternatives with preferably similar potency, but without the side effects through a targeted inhibition of specific pathways (Chapter 3). For this, the overlapping and unique inflammatory pathways activated in different diseases with a chronic inflammationcomponent should be better delineated. That would allow to define a priori a set of (in vitro) inflammation tests that define potency when screening compound libraries. Also, new compounds with various and potent anti-inflammatory activity profiles should be identified.

These alternatives could be found in the imminent field of natural products (Chapter 2 and 3), because of their great structural diversity and alleged anti-inflammatory properties. Plant extracts and mixtures have been used for centuries for diverse ailments in traditional medicine. There have been renewed interest in these products, particularly over the past decades. Nevertheless, plant extracts and mixtures 
are difficult to characterize. They contain thousands of molecules that could have beneficial or detrimental effects to the overall therapeutic outcome. Therefore, it is required that the individual molecules in these extracts that are of importance for the therapeutic effect are identified and validated. These identified molecules are often used as drug leads by pharmaceutical companies and still many validated drugs are based on molecules of natural origin.

To map the status of the natural product field as inspiration for anti-inflammatory medicine, a large systematic review was conducted (Chapter 2). In this review, the objective was to provide predictive parameters that would allow to identify potent anti-inflammatory compounds based on the physicochemical characteristics of the molecules. The literature reviewed formed a substantial library of a variety of structural classes of natural compounds. The strength of natural products as potent drugs or drug leads lies in their structural diversity, and from this 'library' we could distill some general rules for selecting potent anti-inflammatory compounds (please see Bioavailability Rules). Although these rules could be applied to most natural products showing relative good anti-inflammatory activity, there were some exceptions In these cases, potent anti-inflammatory activity was reported that was not predicted based on the molecular properties, for instance rutin was one such compound (80, Tables 2.11 and 8.1). What also emerged from the systematic review is an urgent need for standardization of measurements. For many compounds we were unable to extract from the data provided a clear estimate of the potency. Also, the anti-inflammatory assays that were performed were similar but not the same. These small differences could have significant impact on the interpretation of the results. A recent review article concluded that there is a reproducibility crisis in science [9, 3, 4]. Many compounds with reported in vivo activity are not active when the experiments are repeated in a different laboratory. Standardization of assays and reporting of data could be of significant benefit.

Another way of finding and defining potent anti-inflammatory natural products is by using head-to head benchmarking studies, which is common in the pharmaceutical sciences and drug discovery programs. This principle was followed for eight natural products with described strong anti-inflammatory activity (Chapter 3). Using this method, we could directly compare their anti-inflammatory performance to each other and to the validated drug prednisolone (PLP) albeit for a selected set of inflammatory pathways. Remarkably strong anti-inflammatory profiles were shown by curcumin (Cur) and berberine $(\mathrm{BBCl})$ (Figure 3.4). The broad activity can also be a regarded a point of concern. For example, for curcumin, therapeutic activity had been reported in many diseases and in many different assays [2, 1]. It has been argued that curcumin does not act as a true pharmacological agent but rather as a 
membrane disruptor and there is usually little evidence that curcumin specifically interacts with receptors ([5]).

Drawbacks of these natural products are the therapeutic availability of curcumin and the relative high dosing of berberine. Indeed, if we compare them to the established bioavailability rules (Chapter 2), we quickly learn that some values are just within or even outside the margins (Table 8.1). The lipophilicity (LogD) of 4.12 of curcumin confirms that difficult water solubility.

Table 8.1: Physicochemical properties for bioavailability predication and druggability (calculated/predicted by ChemAxon's Marvin software [6]).

\begin{tabular}{lllll}
\hline Property & Prednisolone & Rutin & Berberine & Curcumin \\
\hline $\mathrm{MW}<500$ & 484.392 & 610.52 & 371.82 & 368.385 \\
$\mathrm{cLogP} \leqslant 5$ & 1.15 & -0.87 & -1.28 & 4.12 \\
logD at $\mathrm{pH} 7.4 \leqslant 5$ & -2.42 & -2.02 & -1.28 & 4.12 \\
tPSA $<70 / 140 \AA$ & 147.2 & 265.52 & 40.80 & 93.06 \\
H-bond_a $\leqslant 10$ & 7 & 16 & 4 & 6 \\
H-bond_d $\leqslant 5$ & 2 & 10 & 0 & 2 \\
r_bonds $\leqslant 10$ & 4 & 6 & 2 & 8 \\
a_rings $\leqslant 3$ & 0 & 3 & 3 & 2 \\
sugar groups $=0$ & 0 & 2 & 0 & 0 \\
\hline
\end{tabular}

The table underlines that some natural products have a predicted poor bioavailability, but they cuold still be potent anti-inflammatory drugs provided that these bioavailability issues can be overcome. In order to enable bioavailability of these natural products and enhance their therapeutic availability or potency, it is desirable to make use of nanoparticles for drug delivery.

An interesting nanoparticle is LDL (Chapter 4 ), which can be isolated from human plasma and subsequently modified to carry a payload (Figure 4.1). Although LDL is known as 'bad cholesterol' and targeting CVD is often done by reducing LDL using statins, it could potentially also be used as a 'Trojan horse', infiltrating the atherosclerotic plaque or the LDL-receptor overexpressing tumor and subsequently release its drug. Given its resemblance of micelles with a large hydrophobic compartment, LDL appears especially suited for delivery of hydrophobic compounds such as curcumin. Although the isolation from plasma allows this nanoparticle to display a difficult ligand such as ApoBioo on the surface to engage in receptor mediated endocytosis, the isolation procedure is quite elaborate. Other more conventional 
nanoparticles for drug delivery include liposomes and synthetic micelles (Figure 5.2).

To unlock the full anti-inflammatory potential of curcumin, four different nanoparticles (LDL, polymeric micelles, Intralipid and liposomes) with a curcumin payload were prepared (Chapter 5). Encapsulation of curcumin was relatively easy and the particles could easily be dissolved in aqueous buffer, making them suitable for IV injections. And as such, the disadvantageous physicochemical properties (Chapter 2), could be corrected for. However, the retention of the curcumin payload in plasma remained a challenge. This is something that is more frequently observed for hydrophobic compounds. The large hydrophobic surface area in plasma leads to efficient extraction of these compounds from nanocarriers. Specific measures have been taken to overcome this extraction. For example core-crosslinked polymeric micelles have been prepared in which drugs are covalently coupled to the hydrophobic domains in the micellar core. As a result, stable encapsulation can be achieved up until the time point that the covalent bond is broken [10].

Although berberine indeed showed promising anti-inflammatory activity in the head-to-head comparison study (Chapter 3), still quite high dosing was necessary to achieve those effects. Nanoparticles are not only good at enhancing the aqueous solubility, but also in boosting the potency. Truly a win-win situation. And thus, berberine was loaded into liposomes (Chapter 6) and studied in a mouse myocardial infarction model. Encapsulated berberine was able to improve the cardiac function in these mice, whereas free berberine or control liposomes were not (Figure 6.6). This confirmed the superiority of nanoparticle use for drug delivery as the free drug was administered at the same concentration, albeit that the solution had to be warmed to $37^{\circ} \mathrm{C}$ to be able to achieve this solubility.

The fact that validated drugs, like prednisolone, often cause many adverse effects, is an important incentive to continuously keep searching for better alternatives and improving the health safety aspects. An important and established aspect are the human health risk assessments pharmaceutical companies have to perform before new drugs can be marketed. However, subordinate to this are the safety concerns for the (aquatic) environment and the threat they indirectly pose to human health. Therefore, in this thesis, the environmental impact of prednisolone was compared with that of berberine (Chapter 7). Indeed, up to an established dose (Table 7.2), a shift in prescription from prednisolone to berberine is favorable for the (aquatic) environment and human health.

In conclusion, chronic inflammation and its related diseases can be linked to all aspects which are studied in this thesis and related to the general concept of MetSyn (Figure 8.1). Inhibition of pro-inflammatory 


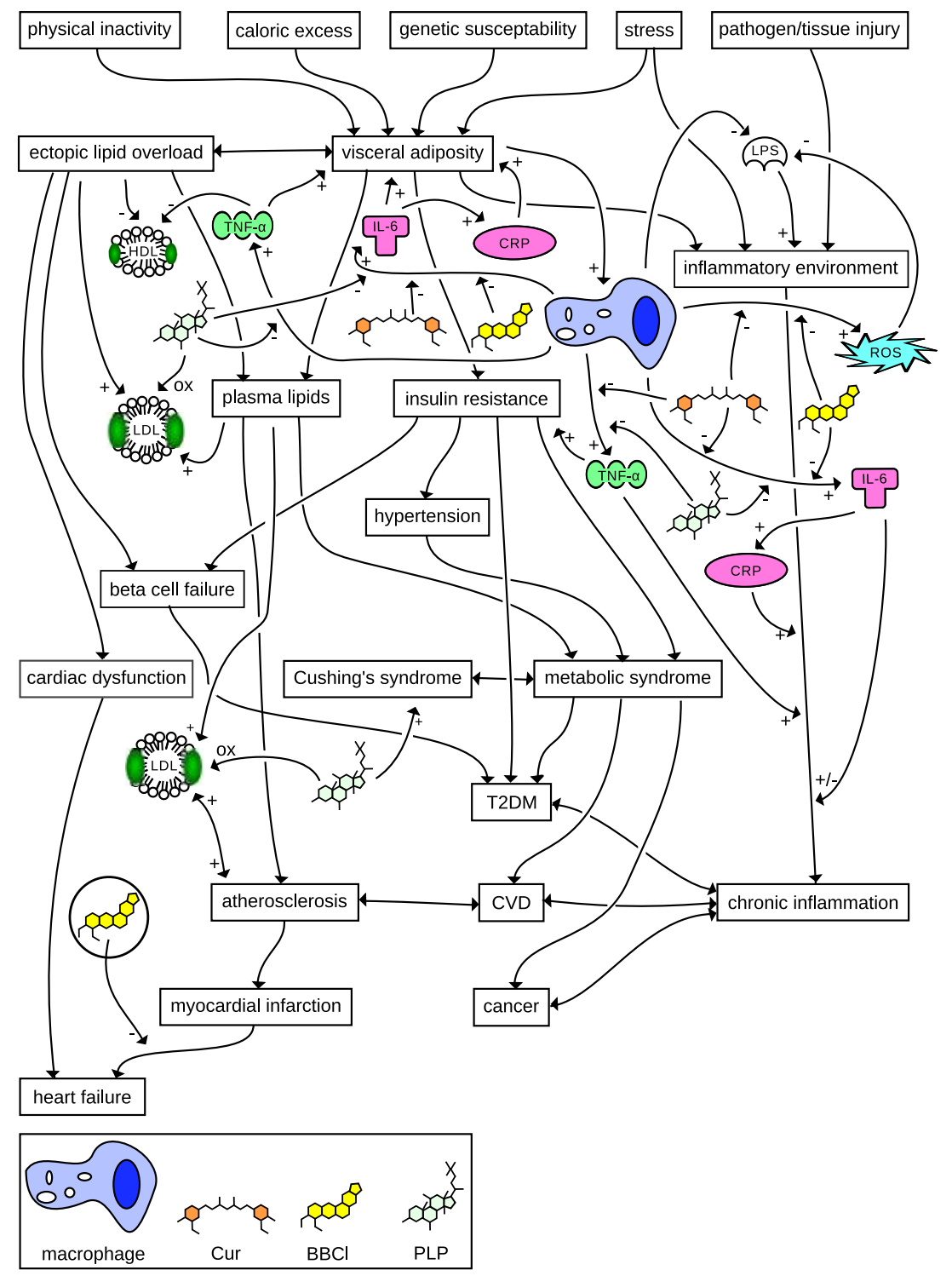

Figure 8.1: Metabolic syndrome schematic. Causes and consequences of metabolic syndrome are closely related to inflammation and chronic inflammatory diseases. T2DM $=$ type 2 diabetes mellitus, $\mathrm{CVD}=$ cardiovascular disease. Inflammatory mediators, antiinflammatory agents and LDL are drawn in according to findings of associated chapters. Cur = curcumin, $\mathrm{BBCl}=$ berberine, PLP = prednisolone. Schematic based on Lusis, 2008 [8] and Kaur, 2014 [7]. 
cytokines, important in acute inflammation, by prednisolone, berberine and curcumin, does also inhibit the onset of chronic inflammation and hence MetSyn and its related diseases. Although prednisolone is indeed a strong pro-inflammatory cytokine inhibitor, its use should be limited since it not only promotes Cushing's Syndrome, and hence enhances the onset of chronic inflammatory diseases, but it also poses a threat to the (aquatic) environment. There is an abundance of chances by combining anti-inflammatory natural products with nanocarriers to arrive at potent nanomedicine.

\section{REFERENCES}

[1] Jonathan B. Baell. "Screening-based translation of public research encounters painful problems." In: ACS Medicinal Chemistry Letters 6.3 (2015), pp. 229-234.

[2] Jonathan B. Baell and Michael A. Walters. "Chemical con artists foil drug discovery." In: Nature 513 (2014), pp. 481-483.

[3] C. Glenn Begley and Lee M. Ellis. "Drug development: Raise standards for preclinical cancer research." In: Nature 483.7391 (2012), pp. 531-533.

[4] Leonard P. Freedman, Iain M. Cockburn, and Timothy S. Simcoe. "The Economics of Reproducibility in Preclinical Research." In: PLOS Biology 13.6 (2015), e1002165.

[5] Helgi I. Ingólfsson et al. "Phytochemicals perturb membranes and promiscuously alter protein function." In: ACS Chemical Biology 9.8 (2014), pp. 1788-1798.

[6] Instant JChem Suite, v15.8.17.o. 2015. URL: http : //Www. chemaxon . com.

[7] Jaspinder Kaur. "A comprehensive review on metabolic syndrome." In: Cardiology Research and Practice 2014 (2014).

[8] Aldons J. Lusis, Alan D. Attie, and Karen Reue. "Metabolic syndrome: from epidemiology to systems biology." In: Nature reviews. Genetics 9.11 (2008), pp. 819-30.

[9] Florian Prinz, Thomas Schlange, and Khusru Asadullah. "Believe it or not: how much can we rely on published data on potential drug targets?" In: Nature reviews. Drug discovery 10.9 (2011), p. 712 .

[10] Cristianne J. Rijcken et al. "Hydrolysable core-crosslinked thermosensitive polymeric micelles: Synthesis, characterisation and in vivo studies." In: Biomaterials 28.36 (2007), pp. 5581-5593. 
Table S1. Physicochemical properties and bioavailability of all natural products and reference compounds per group. The median value (vertical) is given for most properties. CAS RN $=$ Chemical Abstract Services Registry Number, MW = r_bonds = rotatable bowls, a_nings = aromatic rings, sugars = sugar groups

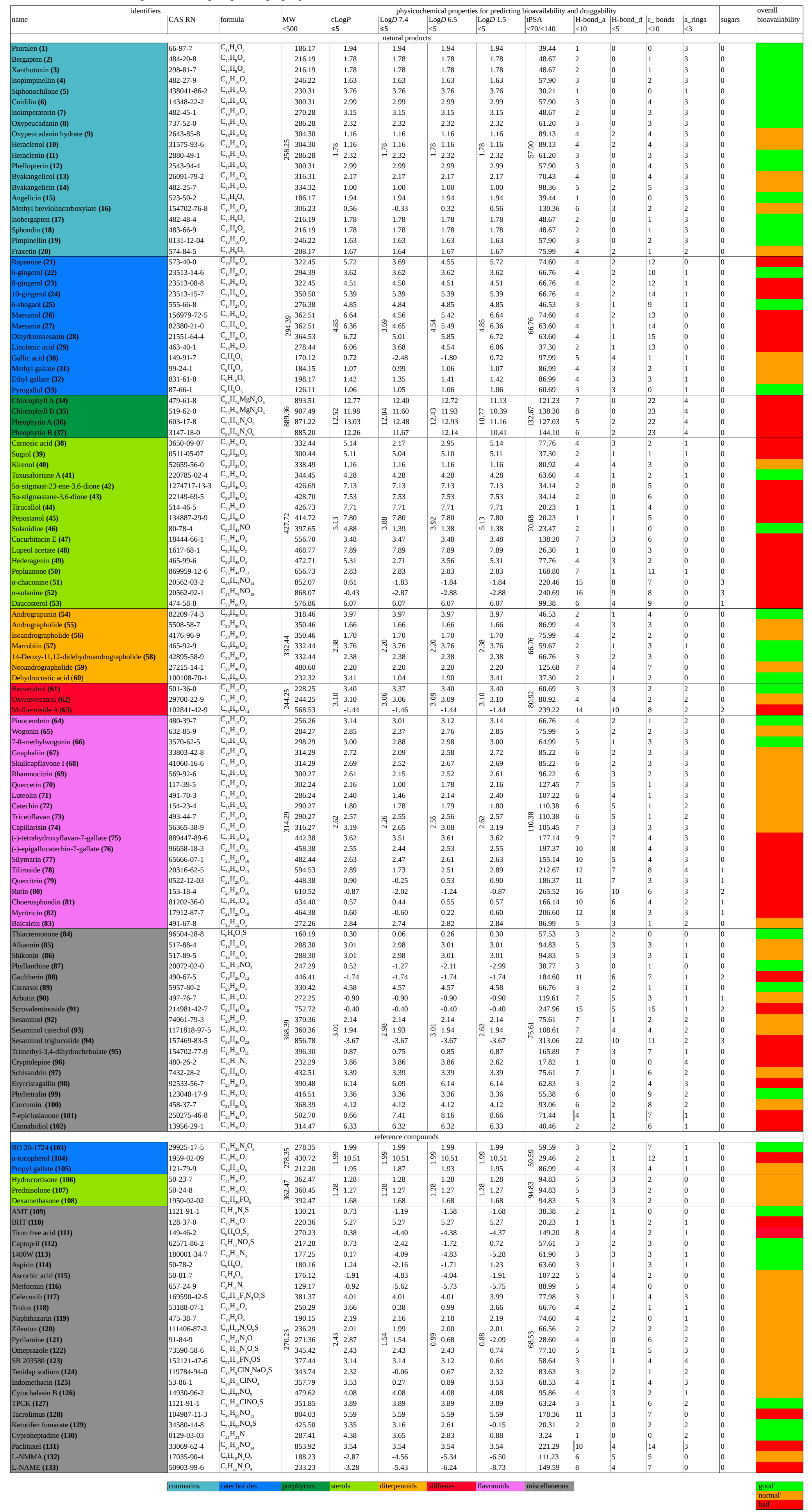


Table S2. Anti-inflammatory activity of all natural products and reference compounds per group. Maximum inhibition $(\%)$ at defined concentration $(\mu M)$ of tested parameters for all compounds in this systematic review. his = histamine, elas = elastase, $m \_$ear $=$mouse ear, $\mathrm{m} \_$_paw $=$mouse paw, $\mathrm{r}$ _paw $=$ rat paw.

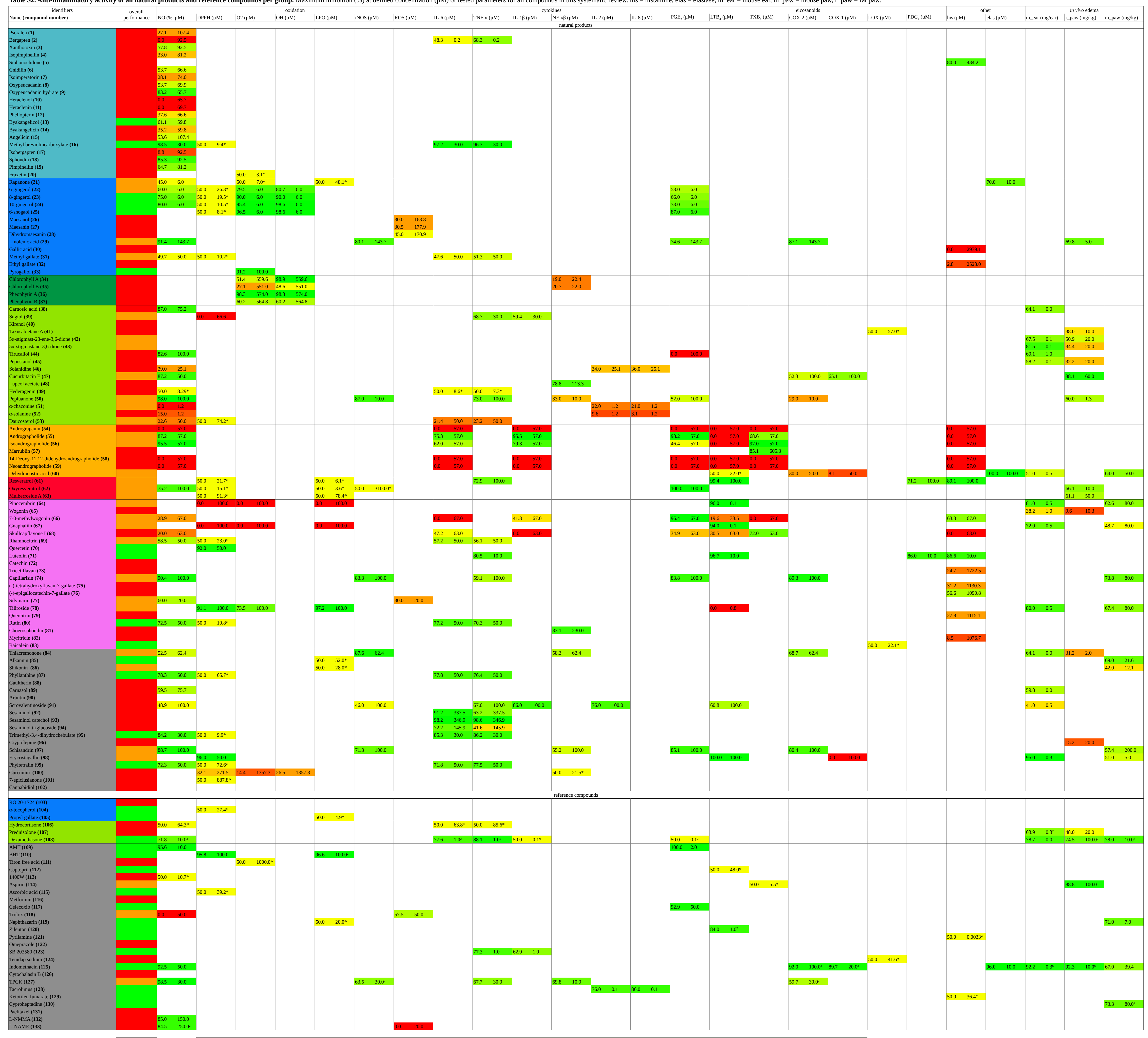

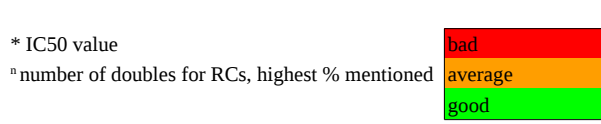

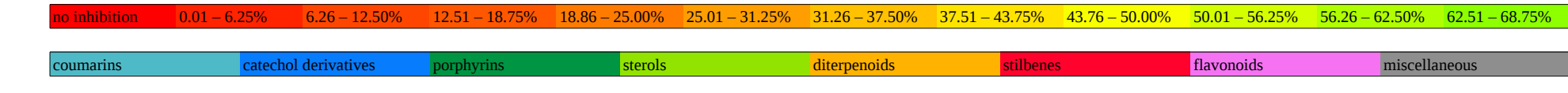



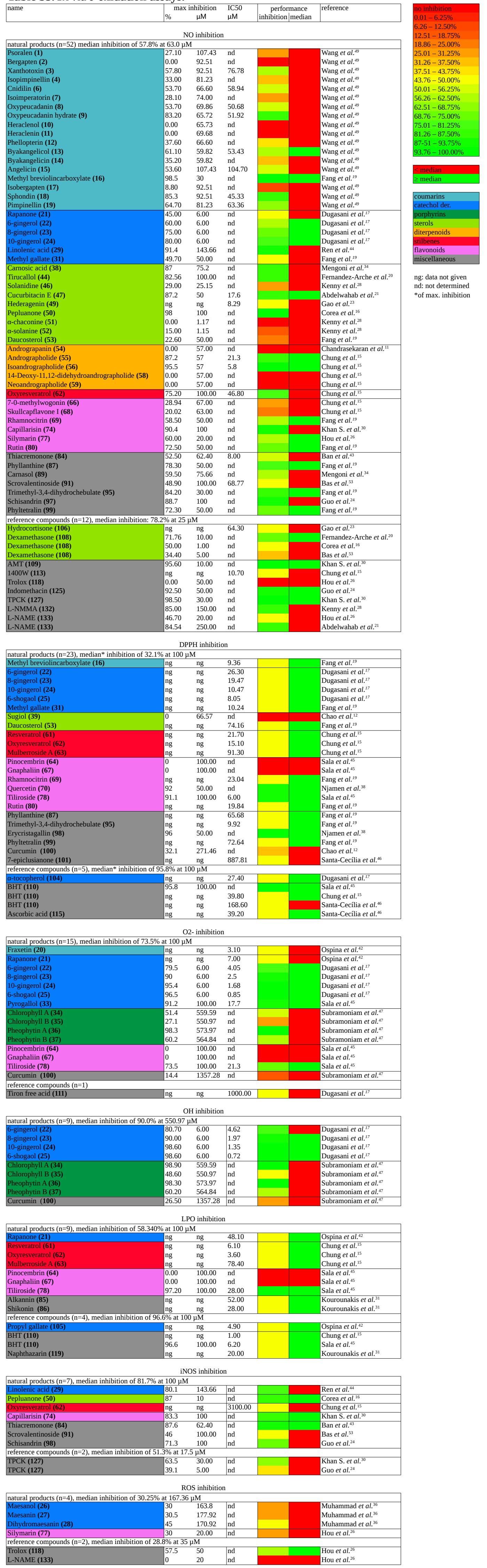
Table S4. In vitro cytokine and chemokine production inhibition assays.

\begin{tabular}{|c|c|c|c|c|c|c|}
\hline \multirow[t]{2}{*}{ name } & \multicolumn{2}{|c|}{ max inhibition } & \multirow{2}{*}{$\begin{array}{l}\text { IC50 } \\
\mu \mathrm{M}\end{array}$} & \multirow{2}{*}{$\begin{array}{c}\text { performance } \\
\text { inhibition median }\end{array}$} & \multirow[t]{2}{*}{ reference } & \multirow{3}{*}{\begin{tabular}{|l} 
no inhibition \\
$0.01-6.25 \%$ \\
$6.26-12.50 \%$ \\
$12.51-18.75 \%$
\end{tabular}} \\
\hline & $\%$ & $\mu \mathrm{M}$ & & & & \\
\hline \multicolumn{6}{|c|}{ IL-6 inhibition } & \\
\hline \multicolumn{6}{|l|}{ natural products $(n=20)$, median inhibiti } & $18.86-25.00 \%$ \\
\hline Bergapten (2) (dimeric) & 48.25 & 0.23 & nd & & Bose et al..$^{54}$ & $25.01-31.25 \%$ \\
\hline Methyl breviolincarboxylate (16) & 97.20 & 30.00 & nd & & Fang et al. ${ }^{19}$ & $31.26-37.50 \%$ \\
\hline Methyl gallate (31) & 47.60 & 50.00 & nd & & Fang et al..$^{19}$ & $37.51-43.75 \%$ \\
\hline Hederagenin (49) & ng & ng & 8.59 & & Gao et al. ${ }^{23}$ & $43.76-50.00 \%$ \\
\hline Daucosterol (53) & 21.40 & 50.00 & nd & & Fang et al. ${ }^{19}$ & $50.01-56.25 \%$ \\
\hline Andrograpanin (54) & 0.00 & 57.00 & nd & & Chandrasekaran et al. ${ }^{11}$ & $56.26-62.50 \%$ \\
\hline Andrographolide (55) & 75.30 & 57.00 & 33.30 & & Chandrasekaran et al. ${ }^{11}$ & $62.51-68.75 \%$ \\
\hline Isoandrographolide (56) & 62.00 & 57.00 & 43.00 & & Chandrasekaran et al. ${ }^{11}$ & $68.76-75.00 \%$ \\
\hline 14-Deoxy-11,12-didehydroandrographolide (58) & 0.00 & 57.00 & nd & & Chandrasekaran et al. ${ }^{11}$ & $75.01-81.25 \%$ \\
\hline Neoandrographolide (59) & 0.00 & 57.00 & nd & & Chandrasekaran et al. ${ }^{11}$ & $81.26-87.50 \%$ \\
\hline 7-0-methylwogonin (66) & 0.00 & 67.00 & nd & & Chandrasekaran et al. ${ }^{11}$ & $87-51-93.75 \%$ \\
\hline Skullcapflavone I (68) & 47.19 & 63.00 & nd & & Chandrasekaran et al. ${ }^{11}$ & $93.76-100.00 \%$ \\
\hline Rhamnocitrin (69) & 57.20 & 50.00 & nd & & Fang et al. ${ }^{19}$ & \\
\hline Rutin (80) & 77.20 & 50.00 & nd & & Fang et al. ${ }^{19}$ & $<$ median \\
\hline Phyllanthine (87) & 77.80 & 50.00 & nd & & Fang et al. ${ }^{19}$ & $\geq$ median \\
\hline Sesaminol (92) & 91.20 & 337.50 & nd & & Jan et al. ${ }^{27}$ & \\
\hline Sesaminol catechol (93) & 98.20 & 346.87 & nd & & Jan et $a l_{.}{ }^{27}$ & coumarins \\
\hline Sesaminol triglucoside (94) & 72.20 & 145.90 & nd & & Jan et al. ${ }^{27}$ & catechol der. \\
\hline Trimethyl-3,4-dihydrochebulate (95) & 85.30 & 30.00 & nd & & Fang et al. ${ }^{19}$ & porphyrins \\
\hline Phyltetralin (99) & 71.80 & 50.00 & nd & & Fang et al. ${ }^{19}$ & sterols \\
\hline \multicolumn{6}{|c|}{ reference compounds $(\mathrm{n}=3$ ), median inhibition of $77.62 \%$ at $1.02 \mu \mathrm{M}$} & diterpenoids \\
\hline Hydrocortisone (106) & ng & ng & 63.80 & & Gao et al. ${ }^{23}$ & stilbenes \\
\hline Dexamethasone (108) & 77.62 & 1.02 & nd & & Bose et al..$^{54}$ & flavonoids \\
\hline Dexamethasone (108) & ng & ng & 0.06 & & Chandrasekaran et al. ${ }^{11}$ & miscellaneous \\
\hline
\end{tabular}

\begin{tabular}{|lllll|l} 
Dexamethasone (108) & ng & ng & 0.06 & & Chandrasekaran et al. \\
\hline
\end{tabular}

\begin{tabular}{|c|c|c|c|c|}
\hline \multicolumn{5}{|c|}{ TNF- $\alpha$ inhibition } \\
\hline \multicolumn{5}{|c|}{ natural products $(\mathrm{n}=19)$, median inhibition of $69.5 \%$ at $50 \mu \mathrm{M}$} \\
\hline Bergapten (2) (dimeric) & 68.33 & 0.23 & nd & Bose et al. ${ }^{54}$ \\
\hline Methyl breviolincarboxylate (16) & 96.30 & 30.00 & nd & Fang et al. ${ }^{19}$ \\
\hline Methyl gallate (31) & 51.30 & 50.00 & nd & Fang et al. ${ }^{19}$ \\
\hline Sugiol (39) & 68.70 & 30.00 & nd & Chao et al. ${ }^{12}$ \\
\hline Hederagenin (49) & ng & ng & 7.32 & Gao et al. ${ }^{23}$ \\
\hline Pepluanone (50) & 73.00 & 100.00 & nd & Corea et al. ${ }^{16}$ \\
\hline Daucosterol (53) & 23.20 & 50.00 & nd & Fang et al. ${ }^{19}$ \\
\hline Resveratrol (61) & 72.90 & 100.00 & nd & Baolin et $a ._{.52}^{52}$ \\
\hline Rhamnocitrin (69) & 56.10 & 50.00 & nd & Fang et al..$^{19}$ \\
\hline Luteolin (71)* & 80.50 & 10.00 & nd & Baolin et al. ${ }^{52}$ \\
\hline Capillarisin (74) & 59.10 & 100.00 & nd & Khan S. et al. ${ }^{30}$ \\
\hline Rutin (80) & 70.30 & 50.00 & nd & Fang et al. ${ }^{19}$ \\
\hline Phyllanthine (87) & 76.40 & 50.00 & nd & Fang et al. ${ }^{19}$ \\
\hline Scrovalentinoside (91) & 67.00 & 100.00 & nd & Bas et al..$^{53}$ \\
\hline Sesaminol (92) & 63.20 & 337.50 & nd & Jan et al. ${ }^{27}$ \\
\hline Sesaminol catechol (93) & 98.60 & 346.87 & nd & Jan et al. ${ }^{27}$ \\
\hline Sesaminol triglucoside (94) & 41.60 & 145.90 & nd & Jan et al. ${ }^{27}$ \\
\hline Trimethyl-3,4-dihydrochebulate (95) & 86.20 & 30.00 & nd & Fang et al. ${ }^{19}$ \\
\hline Phyltetralin (99) & 77.50 & 50.00 & nd & Fang et al. ${ }^{19}$ \\
\hline \multicolumn{5}{|c|}{ reference compounds ( $(\mathrm{n}=5)$, median inhibition of $73.65 \%$ at $1.01 \mu \mathrm{M}$} \\
\hline Hydrocortisone (106) & ng & ng & 85.60 & Gao et $a ._{.}^{23}$ \\
\hline Dexamethasone (108) & 88.13 & 1.02 & nd & Bose et al. ${ }^{54}$ \\
\hline Dexamethasone (108) & 70.00 & 1.00 & nd & Corea et al. ${ }^{16}$ \\
\hline SB 203580 (123) & 77.30 & 1.00 & nd & Chao et al. ${ }^{12}$ \\
\hline TPCK (127) & 67.70 & 30.00 & nd & Khan S. et al. ${ }^{30}$ \\
\hline
\end{tabular}

ng: data not given nd: not determined * used as reference

IL-1 $\beta$ inhibition

\begin{tabular}{|c|c|c|c|c|}
\hline \multicolumn{5}{|l|}{ lucts (n=), median } \\
\hline Sugiol (39) & 59.4 & 30.00 & nd & Chao et al. ${ }^{12}$ \\
\hline Andrograpanin (54) & 0 & 57.00 & nd & Chandrasekaran et al. ${ }^{11}$ \\
\hline Andrographolide (55) & 95.5 & 57 & 12 & Chandrasekaran et al. ${ }^{11}$ \\
\hline Isoandrographolide (56) & 79.3 & 57 & 9.9 & Chandrasekaran et al. ${ }^{11}$ \\
\hline 14-Deoxy-11,12-didehydroandrographolide (58) & 0 & 57.00 & nd & Chandrasekaran et al. ${ }^{11}$ \\
\hline Neoandrographolide (59) & 0 & 57.00 & nd & Chandrasekaran et al. ${ }^{11}$ \\
\hline 7-0-methylwogonin (66) & 41.3 & 67.00 & nd & Chandrasekaran et al. ${ }^{11}$ \\
\hline Skullcapflavone I (68) & 0 & 63.00 & nd & Chandrasekaran et al. ${ }^{11}$ \\
\hline Scrovalentinoside (91) & 86 & 100.00 & 28.65 & Bas et al..$^{53}$ \\
\hline \multicolumn{5}{|c|}{ reference compounds $(n=2)$, median inhibition of $62.9 \%$ at $1 \mu \mathrm{M}$} \\
\hline Dexamethasone (108) & ng & ng & 0.07 & Chandrasekaran et al. ${ }^{11}$ \\
\hline SB 203580 (123) & 62.9 & 1.00 & nd & Chao et al. ${ }^{12}$ \\
\hline
\end{tabular}

$\mathrm{NF}-\mathrm{\kappa} \beta$ inhibition

\begin{tabular}{|c|c|c|c|c|}
\hline \\
\hline \multicolumn{5}{|c|}{ natural products $(\mathrm{n}=8)$, median inhibition of $55.2 \%$ at $62.4 \mu \mathrm{M}$} \\
\hline Chlorophyll A (34) & 19 & 22.38 & nd & Subramoniam et al..$^{47}$ \\
\hline Chlorophyll B (35) & 20.7 & 22.04 & nd & Subramoniam et al..$^{47}$ \\
\hline Lupeol acetate (48) & 78.8 & 213.30 & nd & Subramoniam et al. $^{47}$ \\
\hline Pepluanone (50) & 33 & 10 & nd & Subramoniam et al..$^{47}$ \\
\hline Choerosphondin (81) & 83.1 & 230.00 & nd & Subramoniam et al..$^{47}$ \\
\hline Thiacremonone (84) & 58.3 & 62.40 & nd & Subramoniam et al..$^{47}$ \\
\hline Schisandrin (97) & 55.2 & 100 & nd & Subramoniam et al..$^{47}$ \\
\hline Curcumin (100) & ng & ng & 21.50 & Subramoniam et al..$^{47}$ \\
\hline \multicolumn{5}{|c|}{ reference compounds ( $\mathrm{n}=1$ ) } \\
\hline TPCK (127) & 69.8 & 10.00 & nd & Guo et al. ${ }^{24}$ \\
\hline
\end{tabular}

\begin{tabular}{|c|c|c|c|c|}
\hline \multicolumn{5}{|c|}{ IL-2 } \\
\hline \multicolumn{5}{|c|}{ natural products $(\mathrm{n}=4)$, median inhibition of $28.0 \%$ at $13.16 \mu \mathrm{M}$} \\
\hline Solanidine (46) & 34.00 & 25.15 & nd & Kenny et al. ${ }^{2}$ \\
\hline$\alpha$-chaconine (51) & 22.00 & 1.17 & nd & Kenny et al. ${ }^{2}$ \\
\hline$\alpha$-solanine (52) & 9.60 & 1.15 & nd & Kenny et al. ${ }^{2}$ \\
\hline Scrovalentinoside (91) & 76.00 & 100.00 & 15.74 & Bas et al..$^{53}$ \\
\hline \multicolumn{5}{|c|}{ reference compounds $(\mathrm{n}=1)$} \\
\hline Tacrolimus (128) & 76.00 & 0.10 & nd & Kenny et $a l^{22}$ \\
\hline
\end{tabular}

IL-8

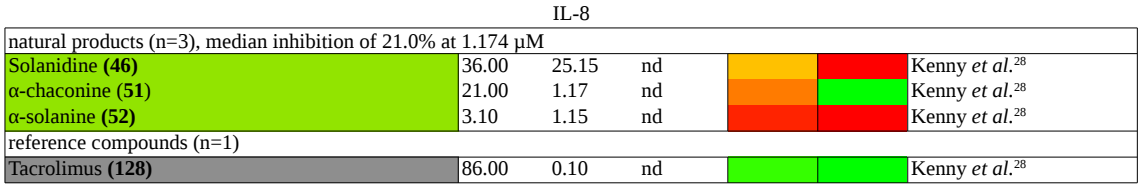


Table S5. In vitro eicosanoids inhibition.

\begin{tabular}{|c|c|c|c|c|c|c|}
\hline name & $\max$ & hibition & IC50 & performance & reference & no inhibition \\
\hline & $\%$ & $\mu \mathrm{M}$ & $\mu \mathrm{M}$ & inhibition median & & $0.01-6.25 \%$ \\
\hline & & $\mathrm{PGE}_{2}$ & & & & $\begin{array}{l}0.20-12.50 \% \\
12.51-18.75 \%\end{array}$ \\
\hline natural products $(\mathrm{n}=17)$, median inhibition of 66 . & at $57.0 \mu$ & & & & & $18.86-25.00 \%$ \\
\hline 6-gingerol (22) & 58.00 & 6.00 & nd & & Dugasani et $a l^{17}$ & $25.01-31.25 \%$ \\
\hline 8-gingerol (23) & 66.00 & 6.00 & nd & & Dugasani et al. ${ }^{17}$ & $31.26-37.50 \%$ \\
\hline 10-gingerol (24) & 73.00 & 6.00 & nd & & Dugasani et $\mathrm{al}^{17}$ & $37.51-43.75 \%$ \\
\hline 6-shogaol (25) & 87.00 & 6.00 & nd & & Dugasani et al..$^{17}$ & $43.76-50.00 \%$ \\
\hline Linolenic acid (29) & 74.60 & 143.66 & nd & & Ren et al. ${ }^{44}$ & $50.01-56.25 \%$ \\
\hline Tirucallol (44) & 0.00 & 100.00 & nd & & Fernandez-Arche et al. ${ }^{20}$ & $56.26-62.50 \%$ \\
\hline Pepluanone (50) & 52.00 & 100.00 & nd & & Corea et al. ${ }^{16}$ & $62.51-68.75 \%$ \\
\hline Andrograpanin (54) & 0.00 & 57.00 & nd & & Chandrasekaran et al. ${ }^{11}$ & $68.76-75.00 \%$ \\
\hline Andrographolide (55) & 98.17 & 57.00 & 23.7 & & Dugasani et al..$^{17}$ & $75.01-81.25 \%$ \\
\hline Isoandrographolide (56) & 46.44 & 57.00 & nd & & Chandrasekaran et al. ${ }^{11}$ & $81.26-87.50 \%$ \\
\hline 14-Deoxy-11,12-didehydroandrographolide (58) & 0.00 & 57.00 & nd & & Chandrasekaran et al. ${ }^{11}$ & $87-51-93.75 \%$ \\
\hline Neoandrographolide (59) & 0.00 & 57.00 & nd & & Chandrasekaran et al. ${ }^{11}$ & $93.76-100.00 \%$ \\
\hline Oxyresveratrol (62) & 100.00 & 100.00 & 19.1 & & Chung et al. ${ }^{15}$ & \\
\hline 7-0-methylwogonin (66) & 96.40 & 67.00 & 19.7 & & Chandrasekaran et al. ${ }^{11}$ & $<$ median \\
\hline Skullcapflavone I (68) & 34.87 & 63.00 & nd & & Chandrasekaran et al. ${ }^{11}$ & $\geq$ median \\
\hline Capillarisin (74) & 83.80 & 100.00 & nd & & Khan S. et al. ${ }^{30}$ & \\
\hline Schisandrin (97) & 85.10 & 100.00 & nd & & Guo et al..$^{24}$ & coumarins \\
\hline reference compounds $(n=4)$, median inhibition of & $45 \%$ at 1 & $\mu \mathrm{M}$ & & & & catechol der. \\
\hline Dexamethasone (108) & 50.00 & 0.12 & nd & & Chandrasekaran et al. ${ }^{11}$ & porphyrins \\
\hline Dexamethasone (108) & 50.00 & 1.00 & nd & & Corea et al. ${ }^{16}$ & sterols \\
\hline AMT (109) & 100.00 & 2.00 & nd & & Dugasani et $a .^{17}$ & diterpenoids \\
\hline Celecoxib (117) & 92.90 & 50.00 & nd & & Dugasani et $\mathrm{al}^{1{ }^{17}}$ & stilbenes \\
\hline & & $\mathrm{LTB}_{4}$ & & & & flavonoids \\
\hline natural products $(n=15)$, median inhibition of 25 . & at $57.0 \mu$ & & & & & miscellaneous \\
\hline Andrograpanin (54) & 0.00 & 57.00 & nd & & Chandrasekaran et al. ${ }^{11}$ & ng: data not given \\
\hline Andrographolide (55) & 0.00 & 57.00 & nd & & Chandrasekaran et al. ${ }^{11}$ & nd: not determined \\
\hline Isoandrographolide (56) & 0.00 & 57.00 & nd & & Chandrasekaran et al. ${ }^{11}$ & * used as reference \\
\hline 14-Deoxy-11,12-didehydroandrographolide (58) & 0.00 & 57.00 & nd & & Chandrasekaran et al. ${ }^{11}$ & \\
\hline Neoandrographolide (59) & 0.00 & 57.00 & nd & & Chandrasekaran et al. ${ }^{11}$ & \\
\hline Dehydrocostic acid (60) & ng & ng & 22.00 & & Hernández et al. ${ }^{25}$ & \\
\hline Resveratrol (61) & 99.40 & 100.00 & nd & & Baolin et al.52 & \\
\hline Pinocembrin (64) & 96.00 & 0.06 & nd & & Sala et al..$^{45}$ & \\
\hline 7-0-methylwogonin (66) & 19.60 & 33.50 & nd & & Chandrasekaran et al. ${ }^{11}$ & \\
\hline Gnaphaliin (67) & 94.00 & 0.06 & nd & & Sala et al. ${ }^{45}$ & \\
\hline Skullcapflavone I (68) & 30.50 & 63.00 & nd & & Chandrasekaran et al. ${ }^{11}$ & \\
\hline Luteolin (71) & 96.70 & 10.00 & nd & & Baolin et $a . .52$ & \\
\hline Tiliroside (78) & 0.00 & 0.84 & nd & & Sala et al. ${ }^{45}$ & \\
\hline Scrovalentinoside (91) & 60.80 & 100.00 & 62.03 & & Bas et al. ${ }^{53}$ & \\
\hline Erycristagallin (98) & 100.00 & 100.00 & 23.40 & & Njamen et al. ${ }^{38}$ & \\
\hline reference compounds $(\mathrm{n}=3$ ), median inhibition of & $85 \%$ at 0 & $5 \mu \mathrm{M}$ & & & & \\
\hline Captopril (112) & ng & ng & 48.00 & & Chandrasekaran et al. ${ }^{11}$ & \\
\hline Zileuton (120) & 84.00 & 1.00 & nd & & Hernández et al. ${ }^{25}$ & \\
\hline Zileuton (120) & 49.70 & 0.50 & nd & & Bas et al. ${ }^{53}$ & \\
\hline & & $\mathrm{TBX}_{2}$ & & & & \\
\hline natural products $(n=8)$, median inhibition of 34.3 & $57.0 \mu \mathrm{s}$ & & & & & \\
\hline Andrograpanin (54) & 0.00 & 57.00 & nd & & Chandrasekaran et al. ${ }^{11}$ & \\
\hline Andrographolide (55) & 68.60 & 57.00 & 45 & & Chandrasekaran et al. ${ }^{11}$ & \\
\hline Isoandrographolide (56) & 97.00 & 57.00 & 25.4 & & Chandrasekaran et al. ${ }^{11}$ & \\
\hline Marrubiin (57) & 85.10 & 605.28 & nd & & Mnonopi et al..$^{35}$ & \\
\hline 14-Deoxy-11,12-didehydroandrographolide (58) & 0.00 & 57.00 & nd & & Chandrasekaran et al. ${ }^{11}$ & \\
\hline Neoandrographolide (59) & 0.00 & 57.00 & nd & & Chandrasekaran et al. ${ }^{11}$ & \\
\hline 7-0-methylwogonin (66) & 0.00 & 67.00 & nd & & Chandrasekaran et al. ${ }^{11}$ & \\
\hline Skullcapflavone I (68) & 72.00 & 63.00 & 27.7 & & Chandrasekaran et al. ${ }^{11}$ & \\
\hline reference compounds $(\mathrm{n}=1)$ & & & & & & \\
\hline Aspirin (114) & ng & $\mathrm{ng}$ & 5.50 & & Chandrasekaran et al. ${ }^{11}$ & \\
\hline & & COX-2 & & & & \\
\hline natural products $(\mathrm{n}=7)$, median inhibition of 68.7 & $\mathrm{t} 100 \mu \mathrm{M}$ & & & & & \\
\hline Linolenic acid (29) & 87.1 & 143.66 & nd & & Ren et al. ${ }^{44}$ & \\
\hline Cucurbitacin E (47) & 52.3 & 100 & 69 & & Abdelwahab et al. ${ }^{21}$ & \\
\hline Pepluanone (50) & 29 & 10 & nd & & Corea et al. ${ }^{16}$ & \\
\hline Dehydrocostic acid (60) & 30 & 50 & nd & & Hernández et al. ${ }^{25}$ & \\
\hline Capillarisin (74) & 89.3 & 100 & nd & & Khan S. et al. ${ }^{30}$ & \\
\hline Thiacremonone (84) & 68.7 & 62.4 & nd & & Ban et al..$^{43}$ & \\
\hline Schisandrin (97) & 80.4 & 100 & nd & & Guo et al. ${ }^{24}$ & \\
\hline reference compounds $(n=4)$, median inhibition of & $1 \%$ at 25 & & & & & \\
\hline Indomethacin (125) & 92 & 100 & nd & & Abdelwahab et al. ${ }^{21}$ & \\
\hline Indomethacin (125) & 48.4 & 20 & nd & & Hernández et al. ${ }^{25}$ & \\
\hline TPCK (127) & 59.7 & 30 & nd & & Khan S. et al. ${ }^{30}$ & \\
\hline TPCK (127) & 29.7 & 5 & nd & & Guo et al. ${ }^{24}$ & \\
\hline & & COX-1 & & & & \\
\hline natural products $(\mathrm{n}=3)$, median inhibition of $8.1 \%$ & $100 \mu \mathrm{M}$ & & & & & \\
\hline Cucurbitacin E (47) & 65.1 & 100 & 90 & & Abdelwahab et al..$^{21}$ & \\
\hline Dehydrocostic acid (60) & 8.1 & 50 & nd & & Hernández et al. ${ }^{25}$ & \\
\hline Erycristagallin (98) & 0 & 100 & nd & & Njamen et al. ${ }^{38}$ & \\
\hline reference compounds ( $\mathrm{n}=2$ ), average inhibition of & $.4 \%$ at 6 & & & & & \\
\hline Indomethacin (125) & 89.7 & 20 & nd & & Hernández et al. ${ }^{25}$ & \\
\hline Indomethacin (125) & 81 & 100 & nd & & Abdelwahab et al. ${ }^{21}$ & \\
\hline & & LOX & & & & \\
\hline natural products ( $\mathrm{n}=2$ ), average inhibition of $50 \%$ & $39.6 \mu \mathrm{M}$ & & & & & \\
\hline Taxusabietane A (41) & ng & ng & 57.00 & & Khan I. et al. ${ }^{29}$ & \\
\hline Baicalein (83) & ng & ng & 22.10 & & Khan I. et al. ${ }^{29}$ & \\
\hline reference compounds ( $\mathrm{n}=1$ ) & & & & & & \\
\hline Tenidap sodium (124) & ng & ng & 41.60 & & Khan I. et al. ${ }^{29}$ & \\
\hline & & $\mathrm{PDG}_{2}$ & & & & \\
\hline & & & & & & \\
\hline Resveratrol (61) & 71.2 & 100.00 & nd & & Baolin et al..$^{52}$ & \\
\hline Luteolin (71)* & 86 & 10.00 & nd & & Baolin et $a ._{.52}^{52}$ & \\
\hline
\end{tabular}


Table S6. In vitro inhibition of other inflammatory mediators.

\begin{tabular}{|c|c|c|c|c|c|}
\hline \multirow[t]{2}{*}{ name } & \multicolumn{2}{|c|}{ max inhibition } & \multirow{2}{*}{$\begin{array}{l}\text { IC50 } \\
\mu \mathrm{M}\end{array}$} & \multirow{2}{*}{$\begin{array}{c}\text { performance } \\
\text { category median }\end{array}$} & \multirow[t]{2}{*}{ reference } \\
\hline & $\%$ & & & & \\
\hline \multicolumn{6}{|c|}{ histamine } \\
\hline \multicolumn{6}{|c|}{ natural products $(\mathrm{n}=17)$, median inhibition of $8.48 \%$ at $100 \mu \mathrm{M}$} \\
\hline Siphonochilone (5) & 80.00 & 434.20 & 245.32 & & Fouche et al.22 \\
\hline Gallic acid (30) & 0.00 & 2939.10 & nd & & Bao et al. ${ }^{51}$ \\
\hline Ethyl gallate (32) & 2.79 & 2523.00 & nd & & Bao et al. ${ }^{51}$ \\
\hline Andrograpanin (54) & 0.00 & 57.00 & nd & & Chandrasekaran et al. ${ }^{11}$ \\
\hline Andrographolide (55) & 0.00 & 57.00 & nd & & Chandrasekaran et al. ${ }^{11}$ \\
\hline Isoandrographolide (56) & 0.00 & 57.00 & nd & & Chandrasekaran et al. ${ }^{11}$ \\
\hline 14-Deoxy-11,12-didehydroandrographolide (58) & 0.00 & 57.00 & nd & & Chandrasekaran et al. ${ }^{11}$ \\
\hline Neoandrographolide (59) & 0.00 & 57.00 & nd & & Chandrasekaran et al. ${ }^{11}$ \\
\hline Resveratrol (61) & 89.10 & 100.00 & nd & & Baolin et al..$^{52}$ \\
\hline 7-0-methylwogonin (66) & 63.30 & 67.00 & 17.80 & & Chandrasekaran et al. ${ }^{11}$ \\
\hline Skullcapflavone I (68) & 0.00 & 63.00 & nd & & Chandrasekaran et al. ${ }^{11}$ \\
\hline Luteolin (71)* & 86.60 & 10.00 & nd & & Baolin et al. ${ }^{52}$ \\
\hline Tricetiflavan (73) & 24.68 & 1722.50 & nd & & Bao et al. ${ }^{51}$ \\
\hline (-)-tetrahydroxyflavan-7-gallate (75) & 31.20 & 1130.30 & nd & & Bao et al. ${ }^{51}$ \\
\hline (-)-epigallocatechin-7-gallate (76) & 56.61 & 1090.80 & nd & & Bao et al. ${ }^{51}$ \\
\hline Quercitrin (79) & 27.79 & 1115.13 & nd & & Bao et al. ${ }^{51}$ \\
\hline Myritricin (82) & 8.48 & 1076.71 & nd & & Bao et al. ${ }^{51}$ \\
\hline \multicolumn{6}{|c|}{ reference compounds ( $\mathrm{n}=2$ ), average inhibition of $18.2 \%$ at $50 \mu \mathrm{M}$} \\
\hline Pyrilamine (121) & ng & ng & 0.0033 & & Fouche et $a .^{22}$ \\
\hline Ketotifen fumarate (129) & ng & ng & 36.40 & & Chandrasekaran et al. ${ }^{11}$ \\
\hline
\end{tabular}

elastase
\begin{tabular}{|l|llll|l|l|}
\hline natural products (n=2), average inhibition is $85 \%$ at $55 \mu \mathrm{M}$ \\
\hline Rapanone (21) & 70 & 10 & 9.8 & & \\
\hline Dehydrocostic acid (60) & 100 & 100 & 43 & & \\
\hline reference compounds (n=1) & 96 & 10 & 3.9 & & \\
\hline Indomethacin (125) & 96 & & & Ospina et al. ${ }^{42}$ \\
\hline
\end{tabular}
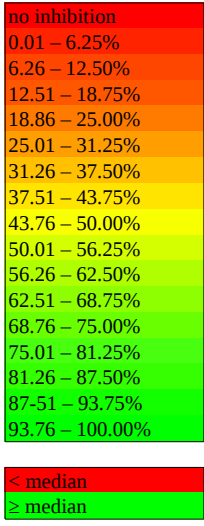

\begin{tabular}{|l|}
\hline coumarins \\
catechol der. \\
porphyrins \\
sterols \\
diterpenoids \\
stilbenes \\
flavonoids \\
miscellaneous \\
\hline
\end{tabular}

ng: data not given nd: not determined * used as reference 
Table S7. In vivo edema reduction.

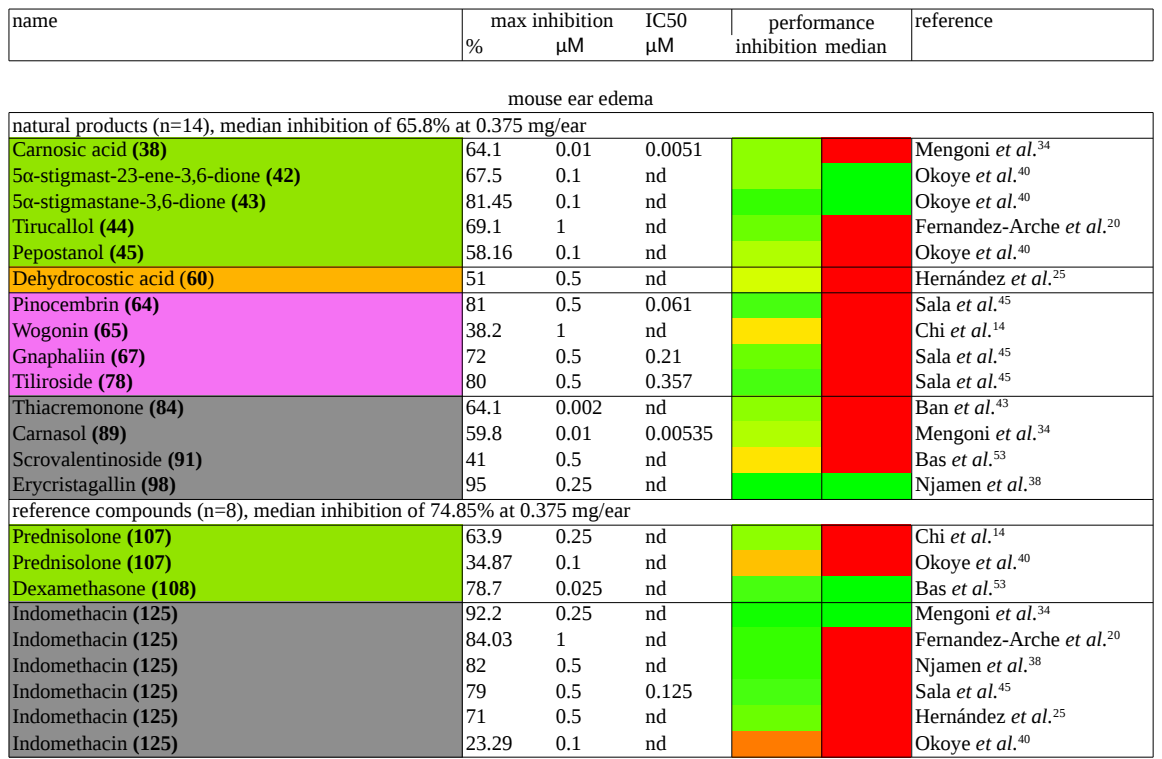

rat paw edema

\begin{tabular}{|c|c|c|c|c|}
\hline \multicolumn{5}{|c|}{ Natural products ( $\mathrm{n}=12$ ), median inhibition of $44.45 \%$ at $15.15 \mathrm{mg} / \mathrm{kg}$} \\
\hline Linolenic acid (29) & 69.8 & 5 & nd & Ren et al. $^{44}$ \\
\hline Taxusabietane A (41) & 38 & 10 & nd & Khan I. et al. ${ }^{29}$ \\
\hline $5 \alpha$-stigmast-23-ene-3,6-dione (42) & 50.9 & 20 & nd & Okoye et al. ${ }^{40}$ \\
\hline $5 \alpha$-stigmastane-3,6-dione (43) & 34.4 & 20 & nd & Okoye et al. ${ }^{40}$ \\
\hline Pepostanol (45) & 32.2 & 20 & nd & Okoye et al..$^{40}$ \\
\hline Cucurbitacin E (47) & 88.1 & 60 & nd & Abdelwahab et al. ${ }^{21}$ \\
\hline Pepluanone (50) & 60 & 1.3 & nd & Corea et al. ${ }^{16}$ \\
\hline Oxyresveratrol (62) & 66.1 & 10 & nd & Chung et $a l . .^{15}$ \\
\hline Mulberroside A (63) & 61.1 & 50 & nd & Chung et al. ${ }^{15}$ \\
\hline Wogonin (65) & 9.6 & 10.3 & nd & Enomoto et al. ${ }^{18}$ \\
\hline Thiacremonone (84) & 31.2 & 2 & nd & Ban et $a .^{43}$ \\
\hline Cryptolepine (96) & 15.18 & 20 & nd & Olajide et al. ${ }^{41}$ \\
\hline \multicolumn{5}{|c|}{ reference compounds ( $\mathrm{n}=9$ ), median inhibition of $66.05 \%$ at $10 \mathrm{mg} / \mathrm{kg}$} \\
\hline Prednisolone (107) & 48 & 20 & nd & Okoye et al. ${ }^{40}$ \\
\hline Dexamethasone (108) & 74.5 & 100 & nd & Enomoto et al. ${ }^{18}$ \\
\hline Dexamethasone (108) & 50 & 0.1 & nd & Corea et al. ${ }^{16}$ \\
\hline Aspirin (114) & 88.8 & 100 & nd & Abdelwahab et al. ${ }^{21}$ \\
\hline Indomethacin (125) & 92.3 & 10 & nd & Chung et al. ${ }^{15}$ \\
\hline Indomethacin (125) & 73.3 & 5 & nd & Khan I. et al. ${ }^{29}$ \\
\hline Indomethacin (125) & 73.1 & 10 & nd & Ren et al. ${ }^{44}$ \\
\hline Indomethacin (125) & 59 & 10 & nd & Ban et al. ${ }^{43}$ \\
\hline Indomethacin (125) & 39.9 & 20 & nd & Okoye et al. ${ }^{40}$ \\
\hline Indomethacin (125) & 13.43 & 10 & nd & Olajide et al. ${ }^{41}$ \\
\hline
\end{tabular}

no inhibition

$6.26-12.50 \%$

$12.51-18.75 \%$

$18.86-25.00 \%$

$25.01-31.25 \%$

$31.26-37.50 \%$

$37.51-43.75 \%$

$43.76-50.00 \%$

$50.01-56.25 \%$

$56.26-62.50 \%$

$62.51-68.75 \%$

$68.76-75.00 \%$

$75.01-81.25 \%$

$81.26-87.50 \%$

$87-51-93.75 \%$

$93.76-100.00 \%$

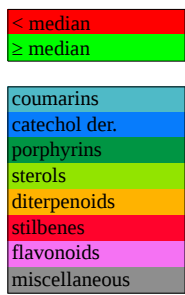

ng: data not given nd: not determined

mouse paw edema

\begin{tabular}{|c|c|c|c|c|}
\hline \multicolumn{5}{|c|}{ Natural products $(\mathrm{n}=9)$, median inhibition of $62.6 \%$ at $80 \mathrm{mg} / \mathrm{kg}$} \\
\hline Dehydrocostic acid (60) & 64 & 50 & 34 & Hernández et al. ${ }^{25}$ \\
\hline Pinocembrin (64) & 62.6 & 80 & nd & Sala et al..$^{45}$ \\
\hline Gnaphaliin (67) & 48.7 & 80 & nd & Sala et al. ${ }^{45}$ \\
\hline Capillarisin (74) & 73.8 & 80 & nd & Khan S. et al. ${ }^{30}$ \\
\hline Tiliroside (78) & 67.4 & 80 & nd & Sala et al. ${ }^{45}$ \\
\hline Alkannin (85) & 69 & 21.6 & nd & Kourounakis et al..$^{31}$ \\
\hline Shikonin (86) & 42 & 12.108 & nd & Kourounakis et al..$^{31}$ \\
\hline Schisandrin (97) & 57.38 & 200 & nd & Guo et al. ${ }^{24}$ \\
\hline Erycristagallin (98) & 51 & 5 & nd & Njamen et al. ${ }^{38}$ \\
\hline \multicolumn{5}{|c|}{ reference compounds $(\mathrm{n}=8)$, median inhibition of $71 \%$ at $10 \mathrm{mg} / \mathrm{kg}$} \\
\hline Dexamethasone (108) & 78 & 10 & nd & Khan S. et al. ${ }^{30}$ \\
\hline Dexamethasone (108) & 77.48 & 1 & nd & Bao et al..$^{51}$ \\
\hline Dexamethasone (108) & 37.7 & 50 & nd & Guo et al. ${ }^{24}$ \\
\hline Naphthazarin (119) & 71 & 7.035 & nd & Kourounakis et $a l^{31}$ \\
\hline Indomethacin (125) & 67 & 39.356 & nd & Kourounakis et al..$^{31}$ \\
\hline Cyproheptadine (130) & 73.3 & 80 & nd & Sala et al..$^{45}$ \\
\hline Cyproheptadine (130) & 66.3 & 5 & nd & Njamen et al. ${ }^{38}$ \\
\hline
\end{tabular}


Table S8. Incomparable or orphan assays.

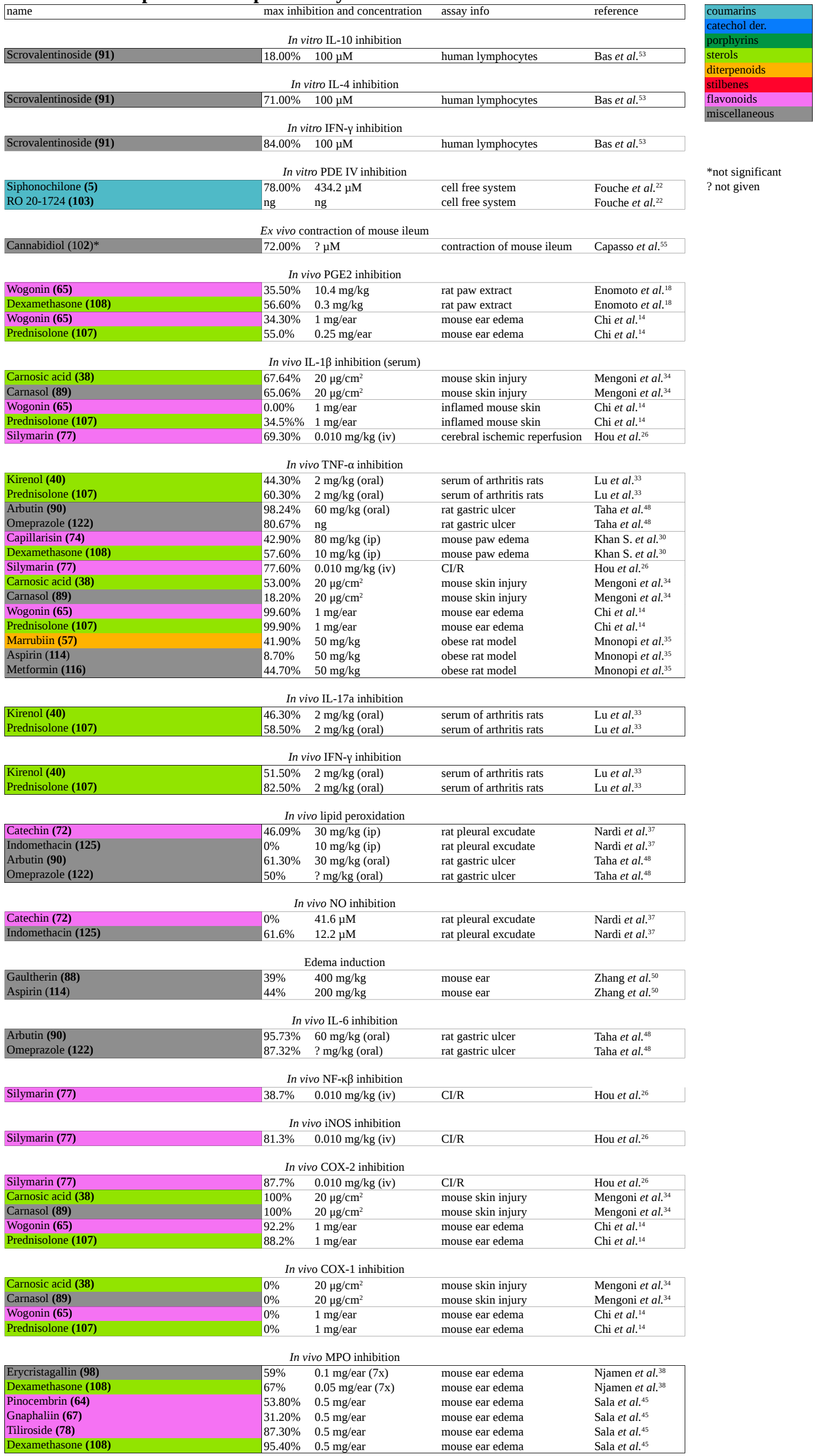


Table S9. Performance of all natural products.
Performance is ordered by both anti-inflammatory

ranking as well as bioavailability. Bioavailability

gradings: 'good' = passing all limits, 'normal' is passing

all limits, but not the blood brain barrier, 'bad' = failing one or more limits.

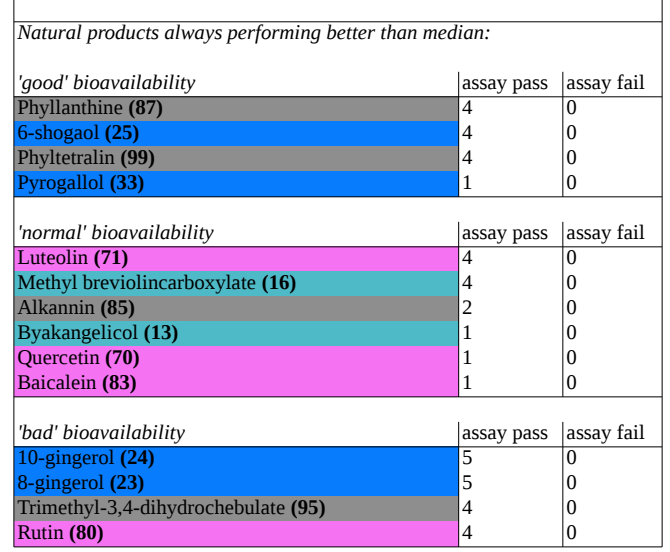

Natural products performing at least one time better than median:

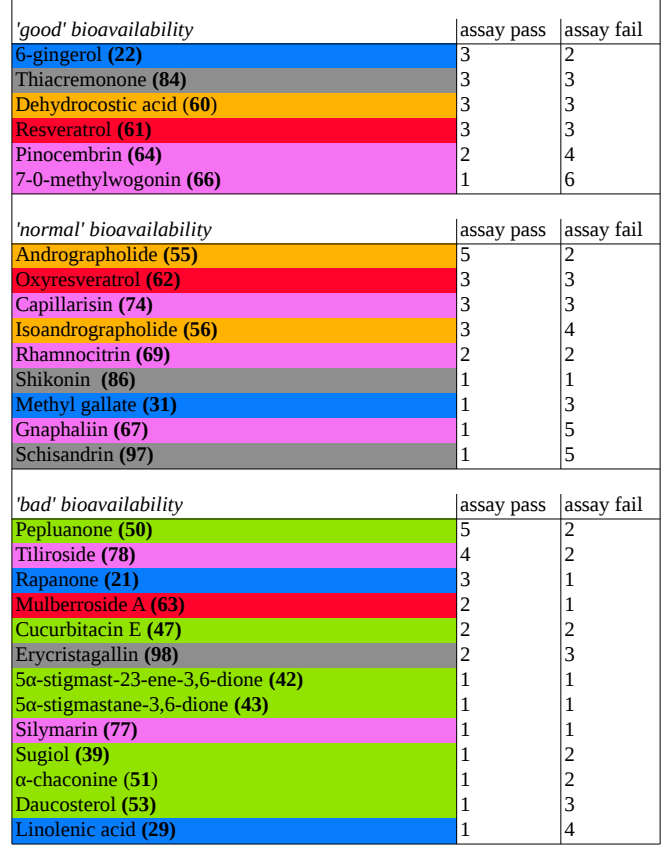

Natural products performing always worse than median:

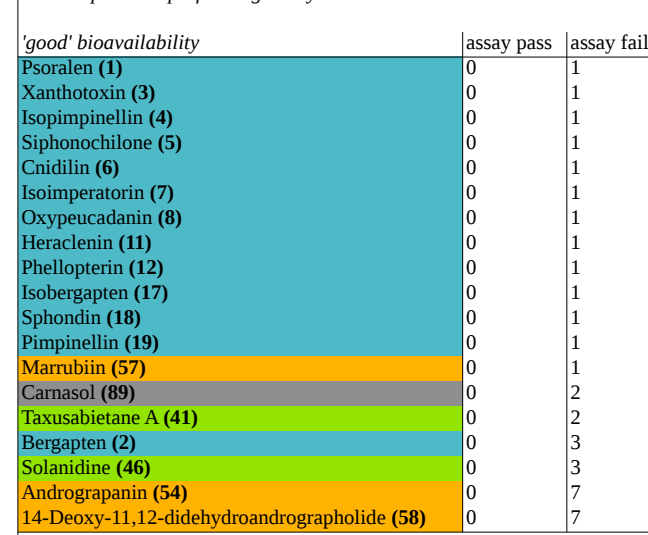

14-Deoxy-11,12-didehydroandrographolide (58)

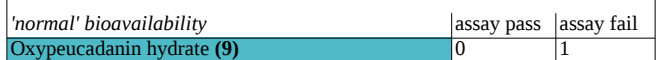

Heraclenol (10)

Byakangelicin (14)
Angelicin (15)

Fraxetin (20)

Gallic acid (30)
Ethyl gallate (32)

Tricetiflavan (73)

Wogonin (65)

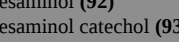

Curcumin (100)

Neoandrographolide (59)

'bad' bioavailability
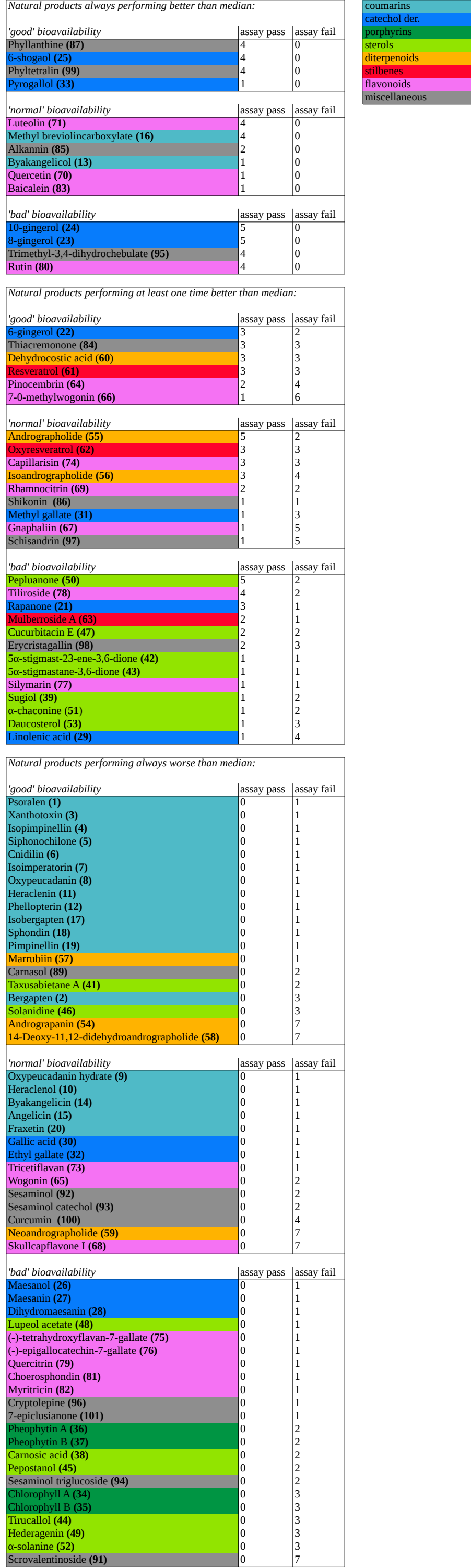

Compounds which were only used in orphan or incomparable assays:

\begin{tabular}{l} 
'normal' bioavailability \\
\hline Kirenol (40)
\end{tabular}

Catechin (72)

Arbutin (90)

'bad' bioavailability

Gaultherin (88)
Cannabidiol (102) 